Supporting Information

\title{
Investigations into the Mechanism of Inter- and Intramolecular Iron-Catalyzed [2+2] Cycloaddition of Alkenes
}

\author{
Matthew V. Joannou, Jordan M. Hoyt, and Paul J. Chirik* \\ Department of Chemistry, Frick Laboratory \\ Princeton University, Princeton, NJ 08544, USA \\ pchirik@princeton.edu
}

\section{Table of Contents:}

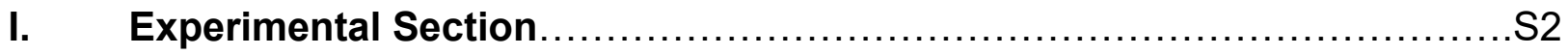

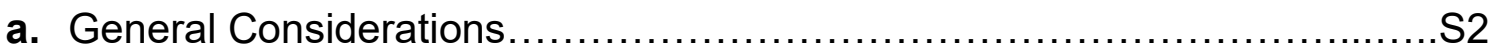

b. Synthesis and Characterization of Disclosed Iron Complexes...............S5

c. Synthesis and Characterization of Organic Products.......................S8

d. Natural Abundance ${ }^{13} \mathrm{C}$ Kinetic Isotope Effect Measurements..............S12

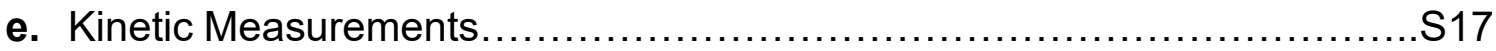

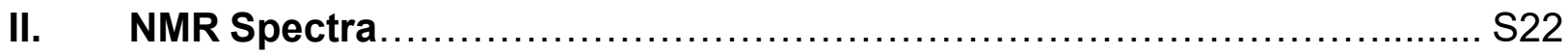

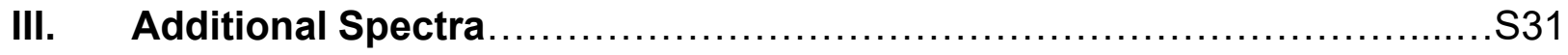

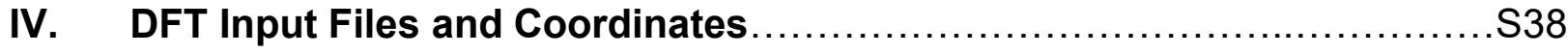

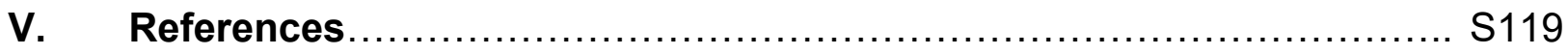




\section{Experimental Section}

\section{- General Considerations}

All air- and moisture-sensitive manipulations were carried out using vacuum line, Schlenk and cannula techniques or in an MBraun inert atmosphere (nitrogen) dry box unless otherwise noted. All glassware was stored in a pre-heated oven $\left(150\right.$ or $\left.180^{\circ} \mathrm{C}\right)$ prior to use. The solvents used for air- and moisture-sensitive manipulations were dried and deoxygenated using literature procedures. ${ }^{1}$ All alpha-olefins and 1,7-octadiene were dried over $\mathrm{LiAlH}_{4}$, degassed via three freeze-pump-thaw cycles, vacuum distilled, and stored over activated $4 \AA$ molecular sieves in a glovebox. 1-deuteroE-1-octene, 1,8-dideutero-E,E-1,7-octadiene, and N,N-bis(3-deutero-Z-allyl)-4-fluoroaniline were prepared according to literature procedures ${ }^{2}$. ${ }^{\text {tric }} \mathrm{PDI}^{3},{ }^{\text {dic }} \mathrm{PDI}^{3},\left({ }^{\text {tric }} \mathrm{PDI}\right) \mathrm{FeCl}_{2}{ }^{4},\left({ }^{\text {dic }} \mathrm{PDI}\right) \mathrm{FeCl}_{2}{ }^{4}$, and $\left({ }^{(\mathrm{Pr}(\mathrm{tb})} \mathrm{PDI}\right) \mathrm{Fe}\left(\mathrm{N}_{2}\right)_{2}{ }^{5}$ were prepared according to literature procedures. $[\mathrm{Na}]_{2}\left[\mathrm{Fe}(\mathrm{CO})_{4}\right]^{6}$ was synthesized according to a literature procedure and 1,4-dibromobutane was dried over $\mathrm{CaH}_{2}$, degassed via three freeze-pump-thaw cycles, vacuum distilled, and stored in a glovebox.

${ }^{1} \mathrm{H}$ NMR spectra were recorded on either Bruker ADVANCE 300 or 500 spectrophotometers operating at $300.13 \mathrm{MHz}$, and $500.46 \mathrm{MHz}$, respectively. ${ }^{13} \mathrm{C}$ NMR spectra were recorded on either Bruker AVANCE 300 or 500 spectrometer operating at $75.48 \mathrm{MHz}$ and $125.85 \mathrm{MHz}$, respectively. All ${ }^{1} \mathrm{H}$ and ${ }^{13} \mathrm{C}$ NMR chemical shifts are reported in ppm relative to $\mathrm{SiMe}_{4}$ using the residual protio solvent peaks as reference (benzene- $d_{6}: 7.16 \mathrm{ppm}$ ) and (benzene- $d_{6}: 128.06 \mathrm{ppm}$ ). ${ }^{1} \mathrm{H}$ NMR data for diamagnetic compounds are reported as follows: chemical shift, multiplicity $(s=$ singlet, $d=$ doublet, $\mathrm{t}=$ triplet, $\mathrm{q}=$ quartet, $\mathrm{p}=$ pentet, $\mathrm{br}=$ broad, $\mathrm{m}=$ multiplet, app = apparent, obsc = obscured), coupling constants $(\mathrm{Hz})$, integration, assignment. ${ }^{1} \mathrm{H}$ NMR data for paramagnetic compounds are reported as follows: chemical shift, integration, peak width at half height $(\mathrm{Hz}) .{ }^{13} \mathrm{C}$ NMR data for diamagnetic compounds are reported as follows: chemical shift, number of protons attached to carbon (e.g. $\mathrm{CH}_{2}$ ), assignment.

Zero-field ${ }^{57} \mathrm{Fe}$ Mössbauer spectra were recorded on a SEE Co. Mössbauer spectrometer (MS4) at $80 \mathrm{~K}$ in constant acceleration mode. ${ }^{57} \mathrm{Co} / \mathrm{Rh}$ was used as the radiation source. WMOSS 
software $^{7}$ was used for the quantitative evaluation of the spectral parameters (least-squares fitting to Lorentzian peaks). The temperature of the sample was controlled by a Janis Research Co. CCS$850 \mathrm{He} / \mathrm{N}_{2}$ cryostat within an accuracy of $0.3 \mathrm{~K}$. Isomer shifts were determined relative to $\alpha$-iron at 298 K.

GC analyses were performed using a Shimadzu GC-2010 gas chromatograph equipped with a Shimadzu AOC-20s autosampler and a Shimadzu SHRXI-5MS capillary column (15m x 250 $\mu \mathrm{m})$. The instrument was set to an injection volume of $5 \mu \mathrm{L}$, and inlet and detector temperatures of 250 ${ }^{\circ} \mathrm{C}$ and $275^{\circ} \mathrm{C}$, respectively. UHP-grade S3 helium was used as carrier gas with a flow rate of 1.82 $\mathrm{mL} / \mathrm{min}$. The temperature program used was as follows: $60^{\circ} \mathrm{C}$, isothermal $1 \mathrm{~min} ; 15^{\circ} \mathrm{C} / \mathrm{min}$ to 250 ${ }^{\circ} \mathrm{C}$, isothermal 2 min. GC-MS analyses were performed on an Agilent 7890A gas chromatograph equipped with an Agilent 5975 mass selective detector (electrospray ionization method), using a RESTEC Rtx®-35MS, Crossbond® 35\% diphenyl-65\% dimethylpolysiloxane column (30 m, 0.32 $\mathrm{mmID}, 0.5 \mu \mathrm{m} \mathrm{df}$ ). The instrument was set to an injection volume of $0.5 \mu \mathrm{L}$ (sample dissolved in dichloromethane) with inlet and detector temperatures of $250^{\circ} \mathrm{C}$. UHP-grade S3 helium was used as carrier gas with a flow rate of $1.82 \mathrm{~mL} / \mathrm{min}$. The temperature program was as follows: $30{ }^{\circ} \mathrm{C}, 5$ ${ }^{\circ} \mathrm{C} / \mathrm{min}$ to $50{ }^{\circ} \mathrm{C}$, isothermal $5 \mathrm{~min}, 10^{\circ} \mathrm{C} / \mathrm{min}$ to $225^{\circ} \mathrm{C}$. LC-MS analyses were performed on an Agilent 6220 liquid chromatography/mass spectrometry (LC/MS) using electrospray ionization timeof-flight (ESI-TOF). Isotopologue percentage calculations were corrected for natural abundance levels of ${ }^{13} \mathrm{C},{ }^{15} \mathrm{~N}$, and ${ }^{2} \mathrm{H}$ (aided by the software package Isopro3).

Elemental analyses were performed at Robinson Microlit Laboratories, Inc., in Ledgewood, NJ. Solid-state magnetic moments were determined using a Johnson Matthey Magnetic Susceptibility Balance that was calibrated with $\mathrm{HgCo}(\mathrm{SCN})_{4}$. High-resolution mass spectra were obtained at Princeton University mass spectrometry facilities using an Agilent 6210 TOF LC/MS. Infrared spectroscopy was conducted on a Thermo-Nicolet iS10 FT-IR spectrometer calibrated with a polystyrene standard. 
Single crystals suitable for X-ray diffraction were coated with polyisobutylene oil in a drybox, transferred to a nylon loop and then quickly transferred to the goniometer head of a Bruker SMART APEX DUO diffractometer equipped with a molybdenum X-ray tube $(\lambda=0.71073 \AA)$ and a Cu X-ray tube $(\lambda=1.54178 \AA)$. Preliminary data revealed the crystal system. The data collection strategy was optimized for completeness and redundancy using the Bruker COSMO software suite. The space group was identified, and the data were processed using the Bruker SAINT+ program and corrected for absorption using SADABS. The structures were solved using direct methods (SHELXS) completed by subsequent Fourier synthesis and refined by full-matrix least-squares procedures.

All DFT calculations were performed with the ORCA package in the gas phase. ${ }^{8}$ Geometry optimizations and single-point calculations were carried out at the B3LYP level of DFT.9,10,11 This hybrid functional often outperforms pure gradient-corrected functionals in the accurate representation of transition metal complexes, especially those involving significant metal-ligand covalency. ${ }^{12}$ Alrichs' all-electron Gaussian basis sets were employed for all calculations, ${ }^{13,14,15}$ wherein the triple- $\zeta$ basis set def2-TZVP, which includes one set of polarization functions, was used to describe metal atoms and all atoms directly coordinated to a metal center. The double- $\zeta$ basis set def2-SV(P), which includes one set of polarizing $d$-functionals on all non-hydrogen atoms, was used for all other atoms. Auxiliary basis sets were chosen to match the orbital basis. ${ }^{16,17,18}$ The RIJCOSX approximation was used to accelerate the calculations. ${ }^{19,20,21}$ Throughout this manuscript, computational results are described using the broken symmetry approach introduced by Ginsberg ${ }^{22}$ and Noodleman et al. ${ }^{23}$ Because several broken symmetry solutions are spin-unrestricted KohnSham equations may be obtained, the general notation for broken symmetry $(m, n)^{24}$ has been adopted, where $m(n)$ denotes the number of spin-up (spin-down) electrons at the two interacting fragments. ${ }^{25}$ Representations of canonical orbitals and the corresponding spin density plots were generated with the program Chimera. ${ }^{26}$ The SCF calculations were tightly converged (TightSCF) with unrestricted spin (UKS) and geometry optimizations converged normally (Opt). Numerical frequency (NumFreq) calculations were performed on each complex to obtain the Gibbs free energy 
(in $\mathrm{kcal} / \mathrm{mol}$ ). All energies are reported as free energies in $\mathrm{kcal} \cdot \mathrm{mol}^{-1}$. Ground states had no imaginary frequencies more negative than $-50 \mathrm{~cm}^{-1}$. Molecular orbitals were derived from the Löwdin Reduced Orbital Populations.

\section{- Synthesis of Disclosed Iron Complexes}

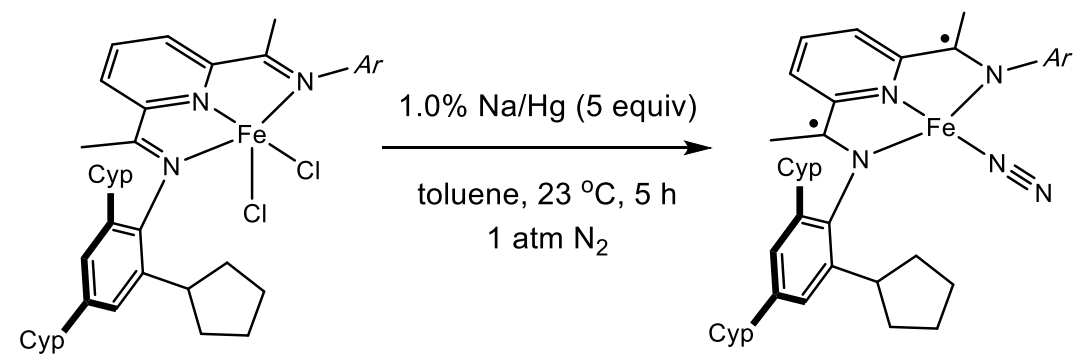

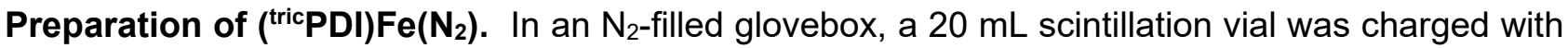
a magnetic stir bar and freshly cut pieces of sodium (34.0 mg, $1.47 \mathrm{mmol})$. Toluene $(5 \mathrm{~mL})$ was added to the vial, followed by neat mercury liquid $(3.40 \mathrm{~g})$. A grey suspension immediately formed which was stirred rapidly at ambient temperature for 15 minutes. The amalgam mixture was then added to a separate scintillation vial containing a magnetic stir bar, (ricPDI) FeCl 2 (250 mg, 0.295 $\mathrm{mmol}$ ), and toluene $(3 \mathrm{~mL})$ followed by rinsing the amalgam vial with an additional $2 \mathrm{~mL}$ of toluene. The reaction mixture was stirred vigorously at ambient temperature for 5 hours. After allowing the reaction to stand for 5 minutes, the mixture was carefully filtered over a thick $(3.5 \mathrm{~cm})$ pad of Celite on a glass frit. The Celtie pad was washed with toluene $(3 \times 3 \mathrm{~mL})$ and the filtrate concentrated to dryness in vacuo. The resulting greenish-brown solid was transferred to a scintillation vial where it was charged with approximately $1 \mathrm{~mL}$ of cold $\left(-35^{\circ} \mathrm{C}\right)$ pentane and allowed to stand in the freezer ($35^{\circ} \mathrm{C}$ ) for 18 hours. The supernatant was removed and the green solid dried in vacuo to afford the title compound in $75 \%$ yield $\left(177 \mathrm{mg}\right.$ ). Anal. Cald. For $\mathrm{C}_{51} \mathrm{H}_{67} \mathrm{~N}_{5} \mathrm{Fe}: \mathrm{C}, 73.36 ; \mathrm{H}, 8.21 ; \mathrm{N}, 11.74$. Found: C, 73.37; H, 8.56; N, 11.16. ${ }^{1} \mathrm{H}$ NMR (300 MHz, benzene- $\left.d_{6}, 23{ }^{\circ} \mathrm{C}, 1 \mathrm{~atm} \mathbf{N}_{2}\right): \delta 9.52(\mathrm{~s}$, $2 \mathrm{H}), 8.80(\mathrm{~s}, 4 \mathrm{H}), 7.50(\mathrm{~s}, 4 \mathrm{H}), 3.94-2.77(\mathrm{~m}, 8 \mathrm{H}), 2.27-2.13(\mathrm{~m}, 8 \mathrm{H}), 1.87\left(\mathrm{ddd},{ }^{3} \mathrm{~J}_{\mathrm{HH}}=16.4,8.2\right.$, $3.7 \mathrm{~Hz}, 8 \mathrm{H}), 1.68\left(\mathrm{dd},{ }^{3} \mathrm{~J}_{\mathrm{HH}}=7.9,4.5 \mathrm{~Hz}, 4 \mathrm{H}\right), 1.57-1.44(\mathrm{~m}, 4 \mathrm{H}), 1.35\left(\mathrm{tq},{ }^{3} \mathrm{~J}_{\mathrm{HH}}=14.9,8.6,6.3 \mathrm{~Hz}\right.$, $12 \mathrm{H}), 0.95(\mathrm{~s}, 4 \mathrm{H}), 0.73-0.52(\mathrm{~m}, 6 \mathrm{H}),-1.30(\mathrm{~s}, 4 \mathrm{H}) .{ }^{1} \mathrm{H}$ NMR $\left(300 \mathrm{MHz}\right.$, benzene- $d_{6}, 23{ }^{\circ} \mathrm{C}, 0$ atm 
$\left.\mathbf{N}_{2}\right): \delta 12.32\left(\mathrm{~d},{ }^{3} \mathrm{~J}_{\mathrm{HH}}=8.1 \mathrm{~Hz}, 2 \mathrm{H}\right), 8.89\left(\mathrm{t},{ }^{3} \mathrm{~J}_{\mathrm{HH}}=7.4 \mathrm{~Hz}, 1 \mathrm{H}\right), 7.25(\mathrm{~s}, 4 \mathrm{H}), 3.52\left(\mathrm{~d},{ }^{3} \mathrm{~J}_{\mathrm{HH}}=36.0 \mathrm{~Hz}\right.$, $3 \mathrm{H}), 3.13\left(\mathrm{q},{ }^{3} \mathrm{~J}_{\mathrm{HH}}=8.8,8.2 \mathrm{~Hz}, 2 \mathrm{H}\right), 2.90(\mathrm{~d}, 4 \mathrm{H}), 2.49(\mathrm{~s}, 2 \mathrm{H}), 2.29\left(\mathrm{dp},{ }^{3} \mathrm{~J}_{\mathrm{HH}}=30.8,7.6 \mathrm{~Hz}, 8 \mathrm{H}\right)$, $2.00-1.59(\mathrm{~m}, 18 \mathrm{H}), 1.57-1.23(\mathrm{~m}, 12 \mathrm{H}), 0.87\left(\mathrm{dd},{ }^{3} \mathrm{~J}_{\mathrm{HH}}=12.4,6.3 \mathrm{~Hz}, 4 \mathrm{H}\right), 0.71-0.54(\mathrm{~m}, 4 \mathrm{H})$, $0.24\left(\mathrm{q},{ }^{3} \mathrm{~J}_{\mathrm{HH}}=9.3,8.7 \mathrm{~Hz}, 4 \mathrm{H}\right),-6.05(\mathrm{~s}, 6 \mathrm{H}) . \quad \mathrm{IR}\left(\mathrm{KBr}, 23^{\circ} \mathbf{C}, \mathbf{c m}^{-1}\right): 2036(\mathrm{~N}=\mathrm{N}, 4$-coordinate $)$. IR (benzene, $\left.23^{\circ} \mathrm{C}, \mathbf{c m}^{-1}\right):$ 2125, $2057(\mathrm{~N}=\mathrm{N}, 5$-coordinate); 2038 (N=N, 4-coordinate). Zero-Field ${ }^{57} \mathrm{Fe}$ Mössbauer (solid state, $80 \mathrm{~K}$ ): $\delta=0.39 \mathrm{~mm} / \mathrm{s},|\Delta \mathrm{E}|=2.07 \mathrm{~mm} / \mathrm{s}$, contains $10 \%$ (rricPDI)Fe( $\left.\mathbf{N}_{2}\right)_{2}$.
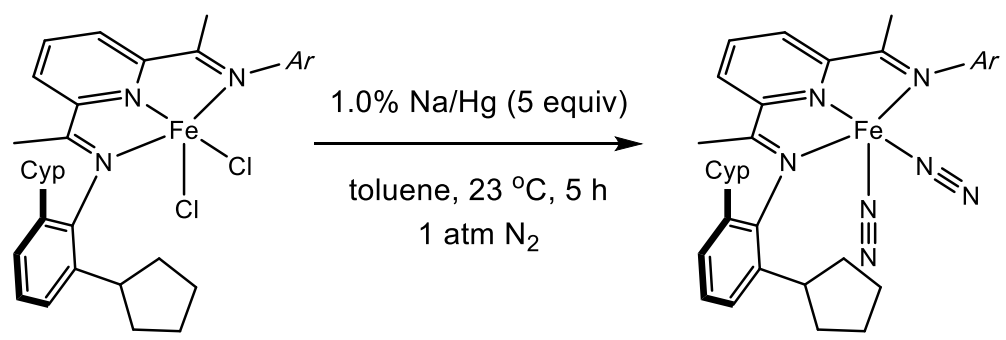

Preparation of ( ${ }^{\text {dic } P D I) F e}\left(\mathbf{N}_{2}\right)$. This complex was prepared in a manner identical to (tricPDI)Fe( $\left.\mathrm{N}_{2}\right)$, but with sodium (42.0 mg, $1.80 \mathrm{mmol})$, mercury (4.20 g), (dicPDI)FeCl 2 (260 mg, $0.365 \mathrm{mmol})$, and $11 \mathrm{~mL}$ of toluene. After trituration from pentane, the title compound was isolated as a dark green solid in $73 \%$ yield $(187 \mathrm{mg})$. Anal. Cald. For $\mathrm{C}_{41} \mathrm{H}_{51} \mathrm{~N}_{7} \mathrm{Fe}: \mathrm{C}, 73.53 ; \mathrm{H}, 7.68 ; \mathrm{N}, 10.46$. Found: $\mathrm{C}$, 73.85; H, 7.58; N, 10.38. ${ }^{1} \mathrm{H}$ NMR (400 MHz, benzene-d $\left.6,23{ }^{\circ} \mathrm{C}\right): \delta 9.46\left(\mathrm{~d},{ }^{3} \mathrm{~J}_{\mathrm{HH}}=7.4 \mathrm{~Hz}, 2 \mathrm{H}\right), 8.47$ $(\mathrm{s}, 6 \mathrm{H}), 7.61\left(\mathrm{~d},{ }^{3} \mathrm{~J}_{\mathrm{HH}}=2.0 \mathrm{~Hz}, 4 \mathrm{H}\right), 4.78(\mathrm{~s}, 1 \mathrm{H}), 2.04-1.92(\mathrm{~m}, 1 \mathrm{H}), 1.91-1.78(\mathrm{~m}, 4 \mathrm{H}), 1.70(\mathrm{t}$, $\left.{ }^{3} J_{\mathrm{HH}}=8.7 \mathrm{~Hz}, 4 \mathrm{H}\right), 1.43\left(\mathrm{ddt},{ }^{3} \mathrm{~J}_{\mathrm{HH}}=12.4,8.0,4.8 \mathrm{~Hz}, 4 \mathrm{H}\right), 1.32-1.21(\mathrm{~m}, 4 \mathrm{H}), 1.18-1.09(\mathrm{~m}, 4 \mathrm{H})$, $0.94\left(\mathrm{dq},{ }^{3} \mathrm{~J}_{\mathrm{HH}}=14.9,7.5 \mathrm{~Hz}, 4 \mathrm{H}\right), 0.69-0.46(\mathrm{~m}, 4 \mathrm{H}),-1.44(\mathrm{~s}, 4 \mathrm{H})$. IR (pentane, $\left.23{ }^{\circ} \mathbf{C}, \mathbf{c m}^{-1}\right)$ : 2049 (N三N, 4-coordinate). 

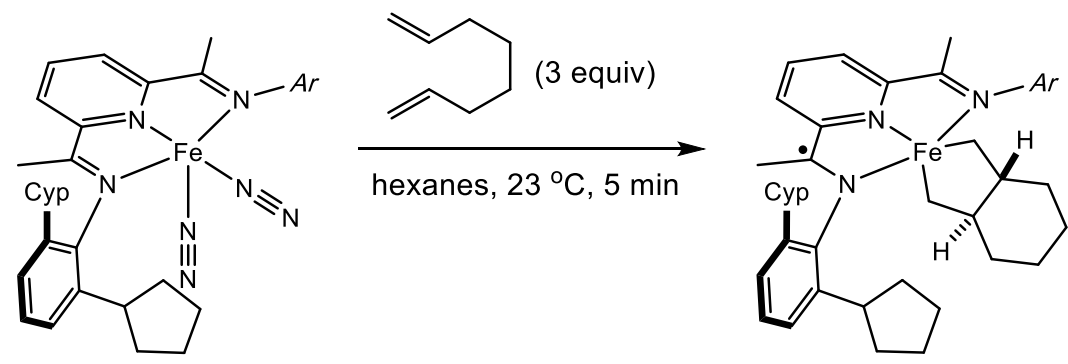

Preparation of (dicPDI)Fe(bimetallacyclo[4.3.0]nonane). In an $\mathrm{N}_{2}$-filled glovebox, a $20 \mathrm{~mL}$ scintillation vial was charged with a magnetic stir-bar, ( $\left.{ }^{\text {dicPDI}}\right) \mathrm{Fe}\left(\mathrm{N}_{2}\right)_{2}(52.0 \mathrm{mg}, 0.0740 \mathrm{mmol})$ and 2 $\mathrm{mL}$ of hexanes. To this suspension was added 1,7-octadiene $(31.5 \mu \mathrm{L}, 0.220 \mathrm{mmol})$ dissolved in 1 $\mathrm{mL}$ of hexanes and the resulting slurry was mixed for 5 minutes. The new solid that formed was collected on a sintered glass frit and washed with pentane $(2 \times 1 \mathrm{~mL})$ to afford the title compound as a red solid in $41 \%$ yield (23 mg). Anal. Cald. For $\mathrm{C}_{49} \mathrm{H}_{65} \mathrm{~N}_{3} \mathrm{Fe}: \mathrm{C}, 78.27 ; \mathrm{H}, 8.71 ; \mathrm{N}, 5.59$. Found: $\mathrm{C}$, 77.97; H, 8.76; N, 5.28. ${ }^{1} \mathrm{H}$ NMR (400 MHz, benzene-d $/ 1$, 7-octadiene, $\left.2{ }^{\circ} \mathrm{C}\right): \delta 120.35\left(\mathrm{~s}, \Delta \mathrm{v}_{1 / 2}=\right.$ $110 \mathrm{~Hz}, 2 \mathrm{H}), 101.90\left(\mathrm{~s}, \Delta \mathrm{v}_{1 / 2}=46 \mathrm{~Hz}, 1 \mathrm{H}\right), 12.45\left(\mathrm{~s}, \Delta \mathrm{v}_{1 / 2}=60 \mathrm{~Hz}, 6 \mathrm{H}\right), 8.08\left(\mathrm{~s}, \Delta \mathrm{v}_{1 / 2}=70 \mathrm{~Hz}, 2 \mathrm{H}\right)$, $6.46\left(\mathrm{~s}, \Delta \mathrm{v}_{1 / 2}=35 \mathrm{~Hz}, 2 \mathrm{H}\right), 5.94\left(\mathrm{~s}, \Delta \mathrm{v}_{1 / 2}=33 \mathrm{~Hz}, 2 \mathrm{H}\right), 4.57\left(\mathrm{~s}, \Delta \mathrm{v}_{1 / 2}=40 \mathrm{~Hz}, 2 \mathrm{H}\right), 4.07\left(\mathrm{~s}, \Delta \mathrm{v}_{1 / 2}=\right.$ $45 \mathrm{~Hz}, 2 \mathrm{H}), 3.28\left(\mathrm{~s}, \Delta \mathrm{v}_{1 / 2}=36 \mathrm{~Hz}, 2 \mathrm{H}\right), 2.67\left(\mathrm{~s}, \Delta \mathrm{v}_{1 / 2}=50 \mathrm{~Hz}, 6 \mathrm{H}\right), 2.24\left(\mathrm{~s}, \Delta \mathrm{v}_{1 / 2}=28 \mathrm{~Hz}, 6 \mathrm{H}\right), 0.88$ $\left(\mathrm{s}, \Delta \mathrm{v}_{1 / 2}=31 \mathrm{~Hz}, \mathrm{H}\right), 0.62\left(\mathrm{~s}, \Delta \mathrm{v}_{1 / 2}=27 \mathrm{~Hz}, 2 \mathrm{H}\right), 0.42\left(\mathrm{~s}, \Delta \mathrm{v}_{1 / 2}=23 \mathrm{~Hz}, 2 \mathrm{H}\right), 0.19\left(\mathrm{~s}, \Delta \mathrm{v}_{1 / 2}=32 \mathrm{~Hz}\right.$ $2 \mathrm{H}),-0.29\left(\mathrm{~s}, \Delta \mathrm{v}_{1 / 2}=35 \mathrm{~Hz}, 2 \mathrm{H}\right),-0.53\left(\mathrm{~s}, \Delta \mathrm{v}_{1 / 2}=33 \mathrm{~Hz}, 2 \mathrm{H}\right),-0.88\left(\mathrm{~s}, \Delta \mathrm{v}_{1 / 2}=46 \mathrm{~Hz}, 4 \mathrm{H}\right),-1.75(\mathrm{~s}$, $\left.\Delta \mathrm{v}_{1 / 2}=62 \mathrm{~Hz}, 2 \mathrm{H}\right),-2.66\left(\mathrm{~s}, \Delta \mathrm{v}_{1 / 2}=29 \mathrm{~Hz}, 2 \mathrm{H}\right),-3.18\left(\mathrm{~s}, \Delta \mathrm{v}_{1 / 2}=22 \mathrm{~Hz}, 2 \mathrm{H}\right),-6.54\left(\mathrm{~s}, \Delta \mathrm{v}_{1 / 2}=51 \mathrm{~Hz}\right.$, $2 \mathrm{H}$ ). Magnetic susceptibility (Evans' Method, $23^{\circ} \mathrm{C}$, benzene- $\boldsymbol{d}_{6}$ ): $2.9(1) \mu_{\mathrm{B}}$.
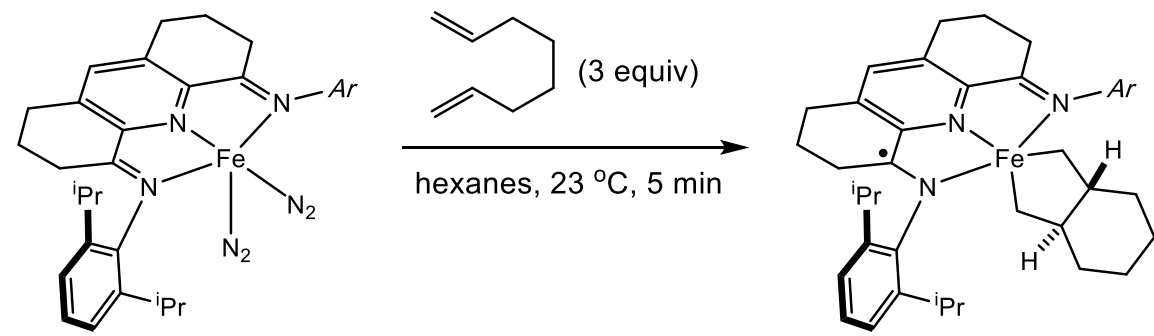

Preparation of ( $\left.{ }^{\mathrm{iPr}(\mathrm{TB})} \mathrm{PDI}\right) \mathrm{Fe}($ bimetallacyclo[4.3.0]nonane). This complex was prepared in a manner identical to ( $\left.{ }^{\text {dic } P D I) F e(b i m e t a l l a c y c l o[4.3 .0] n o n a n e), ~ b u t ~ w i t h ~(~}{ }^{(\mathrm{Pr}(\mathrm{TB})} \mathrm{PDI}\right) \mathrm{Fe}\left(\mathrm{N}_{2}\right)_{2}(90.0 \mathrm{mg}$, 
$0.140 \mathrm{mmol}), 1,7$-octadiene $(58.6 \mu \mathrm{L}, 0.420 \mathrm{mmol})$, and $3 \mathrm{~mL}$ of hexanes. The title compound was isolated as a red solid in $72 \%$ yield $(71 \mathrm{mg})$. Anal. Cald. For $\mathrm{C}_{45} \mathrm{H}_{61} \mathrm{~N}_{3} \mathrm{Fe}: \mathrm{C}, 77.23 ; \mathrm{H}, 8.79 ; \mathrm{N}, 6.00$. Found: C, 77.54; H, 8.75; N, 5.89. ${ }^{1} \mathrm{H}$ NMR (400 MHz, benzene- $\left.d_{6,} \mathbf{2 3}^{\circ} \mathrm{C}\right): \delta 146.03\left(\mathrm{~s}, \Delta \mathrm{v}_{1 / 2}=118\right.$ $\mathrm{Hz}, 1 \mathrm{H}), 48.08\left(\mathrm{~s}, \Delta \mathrm{v}_{1 / 2}=85 \mathrm{~Hz}, 2 \mathrm{H}\right), 42.74\left(\mathrm{~s}, \Delta \mathrm{v}_{1 / 2}=82 \mathrm{~Hz}, 2 \mathrm{H}\right), 11.09\left(\mathrm{~s}, \Delta \mathrm{v}_{1 / 2}=62 \mathrm{~Hz}, 2 \mathrm{H}\right), 7.59$ $\left(\mathrm{s}, \Delta \mathrm{v}_{1 / 2}=35 \mathrm{~Hz}, 3 \mathrm{H}\right), 7.45\left(\mathrm{~s}, \Delta \mathrm{v}_{1 / 2}=39 \mathrm{~Hz}, 3 \mathrm{H}\right), 6.38\left(\mathrm{~s}, \Delta \mathrm{v}_{1 / 2}=27 \mathrm{~Hz}, 2 \mathrm{H}\right), 5.86\left(\mathrm{~s}, \Delta \mathrm{v}_{1 / 2}=16 \mathrm{~Hz}\right.$, $2 \mathrm{H}), 5.19\left(\mathrm{~s}, \Delta \mathrm{v}_{1 / 2}=38 \mathrm{~Hz}, 2 \mathrm{H}\right), 4.65\left(\mathrm{~s}, \Delta \mathrm{v}_{1 / 2}=17 \mathrm{~Hz}, 2 \mathrm{H}\right), 3.95\left(\mathrm{~s}, \Delta \mathrm{v}_{1 / 2}=18 \mathrm{~Hz}, 2 \mathrm{H}\right), 3.07\left(\mathrm{~s}, \Delta \mathrm{v}_{1 / 2}\right.$ $=22 \mathrm{~Hz}, 6 \mathrm{H}), 2.20\left(\mathrm{~s}, \Delta \mathrm{v}_{1 / 2}=18 \mathrm{~Hz}, 6 \mathrm{H}\right), 1.87\left(\mathrm{~s}, \Delta \mathrm{v}_{1 / 2}=20 \mathrm{~Hz}, 6 \mathrm{H}\right), 0.40\left(\mathrm{~s}, \Delta \mathrm{v}_{1 / 2}=93 \mathrm{~Hz}, 3 \mathrm{H}\right), 0.13$ $\left(\mathrm{s}, \Delta \mathrm{v}_{1 / 2}=100 \mathrm{~Hz}, 3 \mathrm{H}\right),-0.40\left(\mathrm{~s}, \Delta \mathrm{v}_{1 / 2}=35 \mathrm{~Hz}, 6 \mathrm{H}\right),-1.33\left(\mathrm{~s}, \Delta \mathrm{v}_{1 / 2}=38 \mathrm{~Hz}, 6 \mathrm{H}\right),-3.54\left(\mathrm{~s}, \Delta \mathrm{v}_{1 / 2}=18\right.$ $\mathrm{Hz}, 2 \mathrm{H}),-9.76\left(\mathrm{~s}, \Delta \mathrm{v}_{1 / 2}=50 \mathrm{~Hz}, 2 \mathrm{H}\right),-11.27\left(\mathrm{~s}, \Delta \mathrm{v}_{1 / 2}=50 \mathrm{~Hz}, 2 \mathrm{H}\right),-12.70\left(\mathrm{~s}, \Delta \mathrm{v}_{1 / 2}=56 \mathrm{~Hz}, 2 \mathrm{H}\right),-$ $13.67\left(\mathrm{~s}, \Delta \mathrm{v}_{1 / 2}=61 \mathrm{~Hz}, 2 \mathrm{H}\right.$ ). Magnetic susceptibility (Evans' Method, $23^{\circ} \mathrm{C}$, benzene- $\left.d_{6}\right): 2.9(1)$ $\mu_{\mathrm{B}}$. Zero-Field ${ }^{57}$ Fe Mössbauer (solid state, $80 \mathrm{~K}$ ): $\delta=0.31 \mathrm{~mm} / \mathrm{s},|\Delta \mathrm{E}|=2.83 \mathrm{~mm} / \mathrm{s}$.

\section{- Synthesis and Characterization of Organic Products}

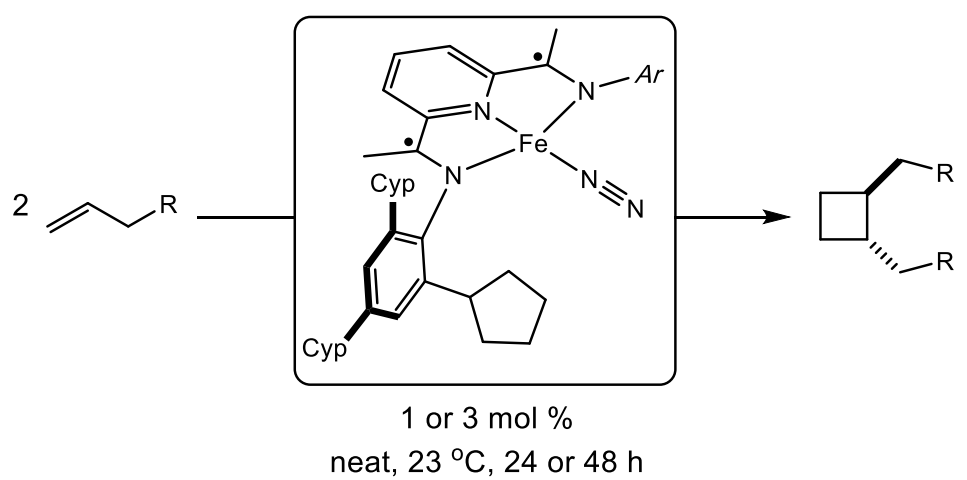

General Intermolecular [2+2] Cycloaddition Procedure: Similar to the procedure described in reference 4. In an $\mathrm{N}_{2}$-filled glovebox, a $1.5 \mathrm{~mL} \mathrm{GC}$ vial was charged with a magnetic stir-bar, (tricPDI)Fe( $\left.\mathrm{N}_{2}\right)$ (1 or $\left.3 \mathrm{~mol} \%\right)$, and the alkene $(1.00 \mathrm{mmol})$. The vial was capped and allowed to stir vigorously for 24 or 48 hours. Upon completion, the vial was removed from the glovebox and charged with approximately $1 \mathrm{~mL}$ of pentane and allowed to stir open to air for 5 minutes. The mixture was filtered through a plug of silica gel and washed copiously with pentane (final filtrate volume approximately $15 \mathrm{~mL}$ ). The filtrate was concentrated in vacuo to afford the trans-1,2-disubstituted 
cyclobutane products. The analytical data of the compounds detailed in Table 1 matched that previously reported.

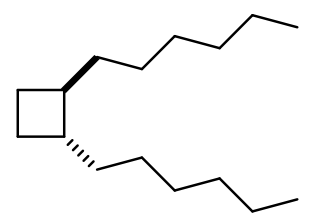

Preparation of trans-1,2-dihexylcyclobutane. Following the general intermolecular [2+2] cycloaddition procedure with (tric PDI)Fe( $\left.\mathrm{N}_{2}\right)(24 \mathrm{mg}, 0.030 \mathrm{mmol})$ and 1-octene $(157 \mu \mathrm{L}, 1.00 \mathrm{mmol})$, the title compound was isolated as a colorless oil in $>98 \%$ yield $(111 \mathrm{mg}) .{ }^{1} \mathbf{H} \mathbf{~ N M R}(600 \mathrm{MHz}$, chloroform- $\left.\boldsymbol{d}_{1}, 2{ }^{\circ} \mathrm{C}\right): \delta 1.97-1.87(\mathrm{~m}, 2 \mathrm{H}), 1.85-1.75(\mathrm{~m}, 2 \mathrm{H}), 1.44\left(\mathrm{dtt},{ }^{3} \mathrm{~J}_{H H}=12.5,5.9,3.6\right.$ $\mathrm{Hz}, 2 \mathrm{H}), 1.41-1.35(\mathrm{~m}, 2 \mathrm{H}), 1.33-1.14(\mathrm{~m}, 8 \mathrm{H}), 0.89\left(\mathrm{t},{ }^{3} J_{H H}=7.0 \mathrm{~Hz}, 3 \mathrm{H}\right) .{ }^{13} \mathrm{C} \mathbf{N M R}(126 \mathrm{MHz}$, chloroform- $\boldsymbol{d}_{1}, \mathbf{2 3}^{\circ} \mathrm{C}$ ): $\delta 42.72,36.91,32.16,29.72,27.55,25.07,22.89,14.30$.

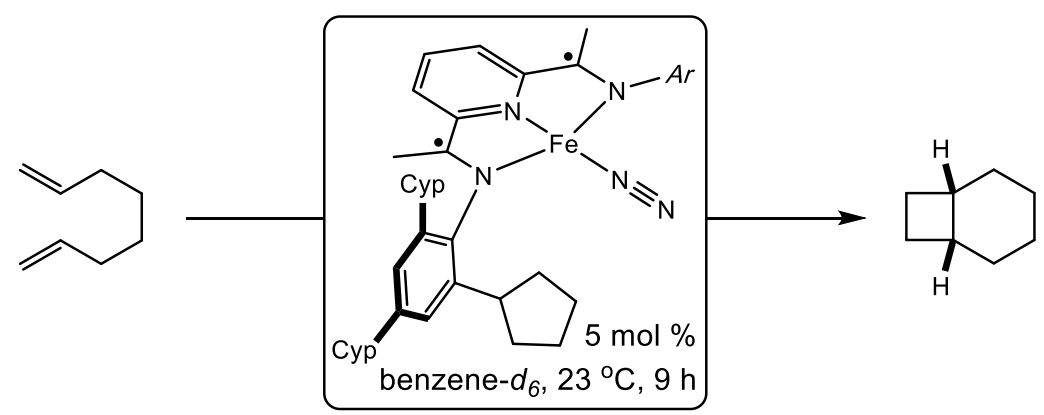

Preparation of cis-bicyclo[4.2.0]octane. In an $\mathrm{N}_{2}$-filled glovebox, (tric PDI)Fe( $\left.\mathrm{N}_{2}\right)(16 \mathrm{mg}, 0.0203$ $\mathrm{mmol}$ ) was added to a J. Young NMR tube, followed by benzene- $d_{6}(600 \mu \mathrm{L})$ and 1,7-octadiene (60 $\mu \mathrm{L}, 0.406 \mathrm{mmol})$. The tube was sealed and then allowed to stand for 9 hours at ambient temperature. The conversion (>98\%) and diastereomeric ratio (92:8 cis:trans) of the product was confirmed by ${ }^{1} \mathrm{H}$ and ${ }^{13} \mathrm{C}$ NMR spectroscopy after tube-to-tube vacuum distillation of the reaction volatiles to a fresh J. Young NMR tube. The analytical data matched that previously reported. ${ }^{27}{ }^{1} \mathbf{H}$ NMR (300 $\mathbf{~ M H z}$, benzene- $\left.d_{6}, 2{ }^{\circ} \mathrm{C}\right): \delta 2.24\left(\mathrm{dtt},{ }^{3} J_{H H}=8.1,5.4,2.9 \mathrm{~Hz}, 2 \mathrm{H}\right), 1.84\left(\mathrm{ddt},{ }^{3} J_{H H}=9.2,6.5,4.4 \mathrm{~Hz}, 2 \mathrm{H}\right)$, $1.75-1.65(\mathrm{~m}, 2 \mathrm{H}), 1.57\left(\mathrm{ddt},{ }^{3} J_{H H}=13.3,7.3,3.8 \mathrm{~Hz}, 2 \mathrm{H}\right), 1.40\left(\mathrm{dddd},{ }^{3} J_{H H}=22.8,10.2,8.5,5.5\right.$ 
$\mathrm{Hz}, 2 \mathrm{H}), 1.23\left(\mathrm{dtd},{ }^{3} \mathrm{JHH}_{\mathrm{HH}}=11.9,6.8,5.1,2.5 \mathrm{~Hz}, 2 \mathrm{H}\right) .{ }^{13} \mathrm{C}$ NMR $\left(126 \mathrm{MHz}\right.$, benzene- $\left.\mathrm{d}_{6}, \mathbf{2 3}^{\circ} \mathrm{C}\right): \delta$ (cis diastereomer): 33.67, 28.49, 25.02, 23.31; (trans diastereomer): 32.17, 29.81, 26.90, 25.92.

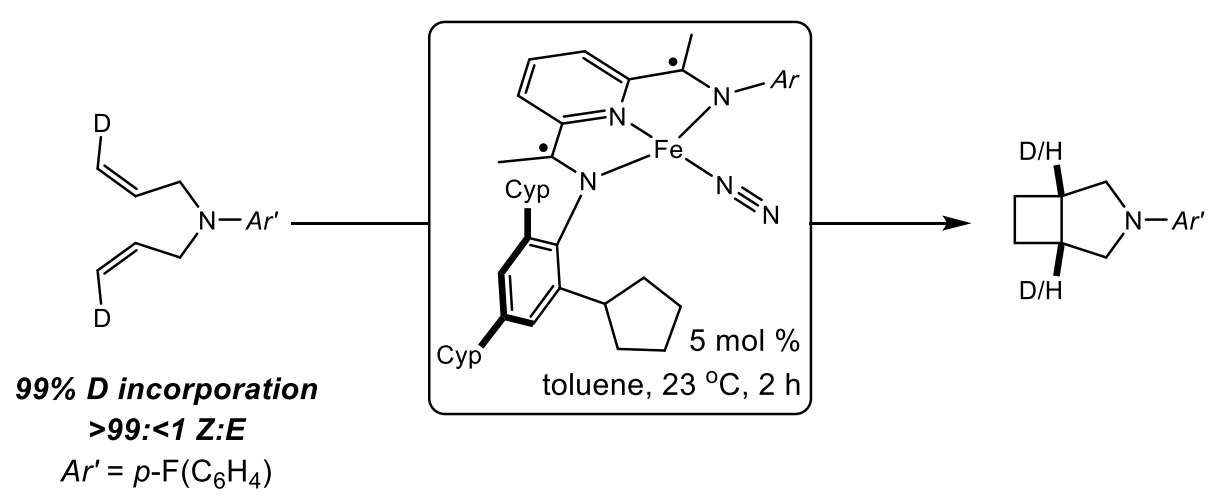

Preparation of cis-3-(4-fluorophenyl)-3-azabicyclo[3.2.0]heptane- $d_{n}$. In an $\mathrm{N}_{2}$-filled glovebox, a $20 \mathrm{~mL}$ scintillation vial was charged with a magnetic stir-bar, (tric PDI)Fe $\left(\mathrm{N}_{2}\right)(9.0 \mathrm{mg}, 0.0111 \mathrm{mmol})$, $\mathrm{N}, \mathrm{N}$-bis(3-deutero-Z-allyl)-4-fluoroaniline $(43.0 \mathrm{mg}, 0.222 \mathrm{mmol})$, and toluene $(7.4 \mathrm{~mL})$. The reaction was stirred for 2 hours at ambient temperature, after which time it was brought out of the glovebox, passed through a plug of silica gel and washed copiously with methylene chloride (final filtrate volume approximately $15 \mathrm{~mL}$ ). The filtrate was concentrated in vacuo to afford the title compound in $>98 \%$ conversion. The analytical data matched that reported previously. ${ }^{1} \mathrm{H}$ NMR $(500 \mathrm{MHz}$, chloroform- $\left.d_{1}, 23^{\circ} \mathrm{C}\right): 6.99(\mathrm{t}, \mathrm{J}=8.8 \mathrm{~Hz}, 2 \mathrm{H}), 6.70\left(\mathrm{dd},{ }^{3} \mathrm{~J}_{\mathrm{HH}}=9.4,4.3 \mathrm{~Hz}, 2 \mathrm{H}\right), 3.48\left(\mathrm{~d},{ }^{3} \mathrm{JHH}_{H}=9.3\right.$ $\mathrm{Hz}, 2 \mathrm{H}), 3.06\left(\mathrm{dt},{ }^{3} \mathrm{~J}_{H H}=6.8,3.6 \mathrm{~Hz}, 2 \mathrm{H}\right), 3.00\left(\mathrm{dd},{ }^{3} \mathrm{~J}_{H H}=9.5,4.8 \mathrm{~Hz}, 2 \mathrm{H}\right), 2.29\left(\mathrm{dt},{ }^{3} \mathrm{~J}_{H H}=9.9,5.9\right.$ $\mathrm{Hz}, 2 \mathrm{H}), 1.83\left(\mathrm{dt},{ }^{3} J_{H H}=9.8,5.0 \mathrm{~Hz}, 2 \mathrm{H}\right)$.
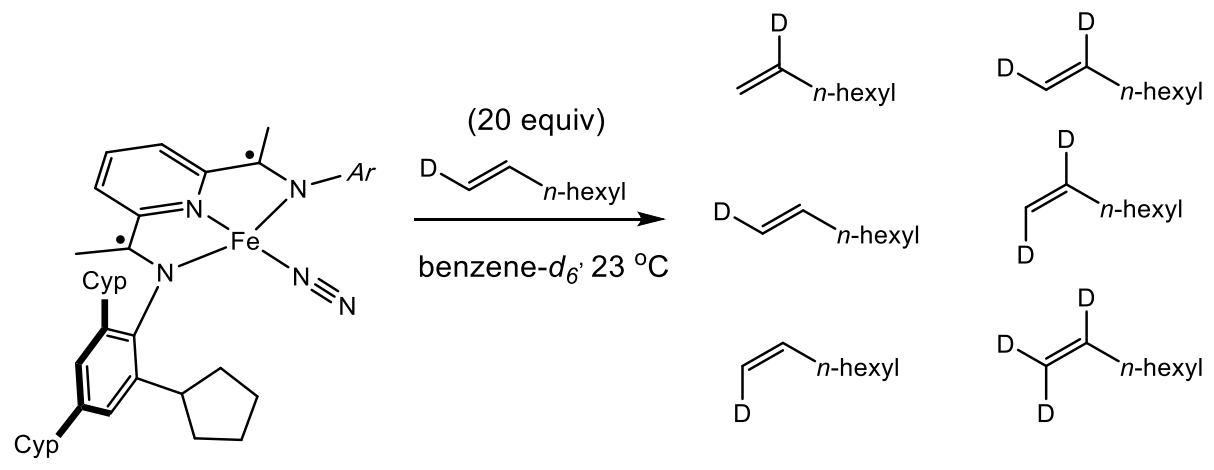
Procedure for Reaction in Scheme 14. In an $\mathrm{N}_{2}$-filled glovebox, a J. Young NMR tube was charged with (rricPDI)Fe( $\left.\mathrm{N}_{2}\right)(10.0 \mathrm{mg}, 0.0124 \mathrm{mmol})$ and benzene- $d_{6}(600 \mu \mathrm{L})$. 1-deutero- $E$-1-octene $(28.0$ $\mathrm{mg}, 0.248 \mathrm{mmol}$ ) was added to the tube, which was then quickly sealed and removed from the glovebox. The reaction was monitored by ${ }^{1} \mathrm{H}$ NMR spectroscopy over the course of 18 hours, which revealed immediate scrambling of the deuterium around the double bond.

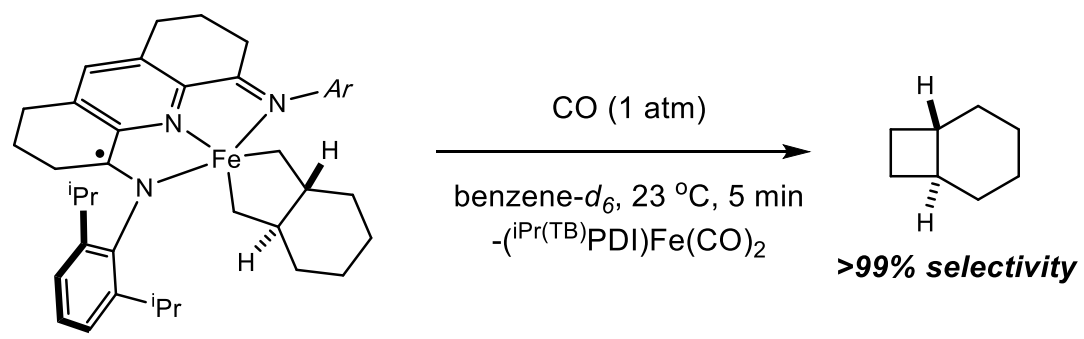

Procedure for Reaction in Scheme 9. In an $\mathrm{N}_{2}$-filled glovebox, a J. Young NMR tube was charged with ( $\left.{ }^{\mathrm{Pr}(\mathrm{TB})} \mathrm{PDI}\right) \mathrm{Fe}$ (bimetallacyclo[4.3.0]nonane) $(8.0 \mathrm{mg}, 0.011 \mathrm{mmol})$ and benzene- $d_{6}(600 \mu \mathrm{L})$, sealed, and then brought out of the glovebox. The solution was frozen at $-196{ }^{\circ} \mathrm{C}$ (liquid nitrogen) and the headspace of the tube removed on a Schlenk line. $1 \mathrm{~atm}$ of carbon monoxide was then introduced into the tube, which was then sealed and thawed in a beaker of cold water. After completion of the reaction, the reaction volatiles were transferred to a fresh J. Young NMR tube via tube-to-tube vacuum distillation and analyzed via ${ }^{1} \mathrm{H}$ NMR spectroscopy, which matched the analytical data previously reported for trans-bicyclo[4.2.0]octane. ${ }^{28}$ The iron residue at the end of the reaction was determined to be $\left({ }^{\mathrm{iPr}(\mathrm{TB})} \mathrm{PDI}\right) \mathrm{Fe}(\mathrm{CO})_{2}$.

Procedure for Freeze-Quench Zero-Field ${ }^{57}$ Fe Mössbauer Studies. In an $\mathrm{N}_{2}$-filled glovebox, a $1.5 \mathrm{~mL}$ GC vial was charged with ( ${ }^{\text {tricPDI}) F e}\left(\mathrm{~N}_{2}\right)(36.0 \mathrm{mg}, 0.0449 \mathrm{mmol})$ and 1-octene (705 $\mu \mathrm{L}, 4.49$ $\mathrm{mmol}$ ). The vial was capped and shaken for 10 minutes (or stirred for 8 hours) at ambient temperature, allowed to settle for 30 seconds, and the solution transferred into a solution Mössbauer cell that was pre-chilled to $-196{ }^{\circ} \mathrm{C}$. After allowing the solution cell to stand in a cold well at $-196{ }^{\circ} \mathrm{C}$ for 15 minutes, it was quickly removed from the glovebox and dunked into a dewar filled with liquid 
nitrogen. The cell was then mounted onto the Mössbauer probe at $-196{ }^{\circ} \mathrm{C}$, which was then placed into the spectrometer cavity at $80 \mathrm{~K}$ under a positive flow of helium. The spectrum was then acquired and processed in the same manner as a solid-state sample.

Procedure for Reaction Monitoring via IR Spectroscopy. In an $\mathrm{N}_{2}$-filled glovebox, a $1.5 \mathrm{~mL} \mathrm{GC}$ vial was charged with a magnetic stir-bar, ( ${ }^{\text {tric } P D I) F e}\left(\mathrm{~N}_{2}\right)(36.0 \mathrm{mg}, 0.0449 \mathrm{mmol})$, and 1-octene $(705$ $\mu \mathrm{L}, 4.49 \mathrm{mmol}$ ). Aliquots (approximately $200 \mu \mathrm{L}$ ) were removed from the vial at 10 minutes, 4 hours, and 18 hours of reaction time and placed into a solution IR cell. The cell was sealed and brought out of the glovebox where the IR spectra were acquired under a positive flow of $\mathrm{N}_{2}$ at $23{ }^{\circ} \mathrm{C}$. A standard spectrum of 1 -octene was subtracted from the reaction spectra after acquisition, although there were no intense stretching bands corresponding to 1 -octene in the $2500-2000 \mathrm{~cm}^{-1}$ region (where the $\mathrm{Fe}-\mathrm{N} \equiv \mathrm{N}$ stretches appear).

\section{n Natural Abundance ${ }^{13} \mathrm{C}$ Kinetic Isotope Effect Measurements}

Preparation of High Conversion Sample (1-octene): Following the general intermolecular [2+2] cycloaddition procedure with (tric PDI)Fe( $\left.\mathrm{N}_{2}\right)(36 \mathrm{mg}, 0.030 \mathrm{mmol})$ and 1-octene $(235 \mu \mathrm{L}, 1.50 \mathrm{mmol})$, the title compound was isolated as a colorless oil in $>98 \%$ yield $(167 \mathrm{mg})$.

Preparation of Low Conversion Sample (1-octene): In an $\mathrm{N}_{2}$-filled glovebox, a $20 \mathrm{~mL}$ scintillation vial containing ( $\left.{ }^{\text {tric } P D I}\right) \mathrm{Fe}\left(\mathrm{N}_{2}\right)(150 \mathrm{mg}, 0.186 \mathrm{mmol})$ was added a magnetic stir-bar and 1-octene (973 $\mu \mathrm{L}, 6.20 \mathrm{mmol})$. After stirring vigorously for 10 minutes, the reaction was quenched by exposure to air outside the glovebox. Conversion of the reaction was determined to be $9 \%$ by gas chromatography with decane as an internal standard. The product was isolated in a similar manner to that described in the general intermolecular [2+2] cycloaddition procedure and as a colorless oil in $9 \%$ yield $(62 \mathrm{mg})$. 
Preparation of High Conversion Sample (1,7-octadiene): In an $\mathrm{N}_{2}$-filled glovebox, ( $\left.{ }^{\text {tric }} \mathrm{PDI}\right) \mathrm{Fe}\left(\mathrm{N}_{2}\right)$ (39.0 $\mathrm{mg}, 0.0480 \mathrm{mmol})$ was added to a J. Young NMR tube, followed by benzene- $d_{6}(480 \mu \mathrm{L})$ and 1,7-octadiene ( $177 \mu \mathrm{L}, 1.20 \mathrm{mmol})$. The tube was sealed and then allowed to stand for 18 hours at ambient temperature. The conversion ( $>98 \%)$ and diastereomeric ratio (92:8 cis:trans) of the product was confirmed by ${ }^{1} \mathrm{H}$ and ${ }^{13} \mathrm{C}$ NMR spectroscopy after tube-to-tube vacuum distillation of the reaction volatiles to a fresh J. Young NMR tube. Theoretical concentration of the NMR sample: $2.5 \mathrm{M}$.

Preparation of Low Conversion Sample (1,7-octadiene): In an $\mathrm{N}_{2}$-filled glovebox, ( $\left.{ }^{\text {tric }} \mathrm{PDI}\right) \mathrm{Fe}\left(\mathrm{N}_{2}\right)$ (78.0 $\mathrm{mg}, 0.0968 \mathrm{mmol}$ ) was added to a J. Young NMR tube, followed by dodecane $(330 \mu \mathrm{L})$, benzene- $d_{6}(100 \mu \mathrm{L})$, and 1,7-octadiene $(361 \mu \mathrm{L}, 2.40 \mathrm{mmol})$. The tube was sealed and inverted for 5 minutes at ambient temperature ( $10 \%$ conversion based on kinetic measurements). The reaction was quenched by addition of neat dibal-H $(641 \mu \mathrm{L}, 3.60 \mathrm{mmol})$, after which time the tube was sealed and removed from the glovebox and heated to $55{ }^{\circ} \mathrm{C}$ for 18 hours (this was done to convert all unreacted 1,7-octadiene to a high-boiling alkyl aluminum species). Upon cooling to ambient temperature, the product was transferred to a capillary NMR tube via a tube-to-tube vacuum distillation. The high-boiling dodecane, $\alpha, \omega$-dialuminumalkyl, and dibal-H remained in the J. Young tube and did not transfer along with the product and benzene- $d_{6}$ in significant amounts. Theoretical concentration of the NMR sample: $2.4 \mathrm{M}$.

Data Acquisition: For NMR measurements of trans-1,2-dihexylcyclobtuane, the high conversion sample was placed into a standard NMR tube along with $370 \mu \mathrm{L}$ of chloroform- $d_{1}$ while the low conversion sample was placed in a capillary NMR tube along with $138 \mu \mathrm{L}$ of chloroform- $d_{1}$. This ensured that the concentration of both NMR samples was 2.0 M. For NMR measurements of cisbicyclo[4.2.0]octane, the high conversion sample was collected in a J. Young NMR tube, while the low conversion sample was collected in a capillary NMR tube (amounts and concentrations detailed in the procedure above). The quantitative ${ }^{13} \mathrm{C}$ NMR spectra were acquired on a Bruker AVANCE 
500 spectrometer operating at $125.85 \mathrm{MHz}$ with inverse gated decoupling and calibrated 90 -degree pulses with a delay time of 120 seconds. Three spectra were recorded for each sample.

All spectra were processed using the MestReNova software package were each FID was zero-filled with 262,144 points. After manual phase correction of each spectrum, a first-order baseline correction was applied fitting a first order polynomial to the non-signal regions and subtracting it from the entire spectrum. The tables below present the integrations, averages, and standard deviations for the signals of interest in each molecule and sample type. ${ }^{13} \mathrm{C}$ KIE values and errors were calculated using equations provided by Singleton and co-workers. ${ }^{29}$ 


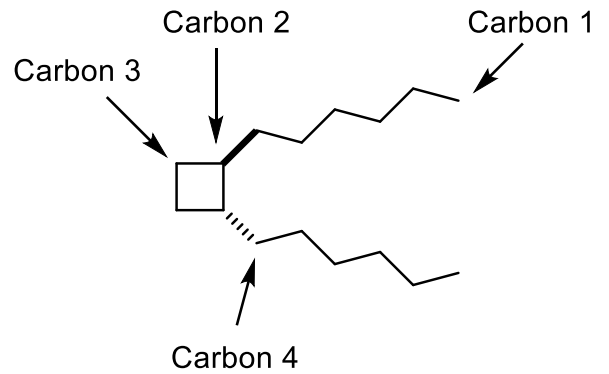

\begin{tabular}{|c|c|c|c|c|}
\hline \multicolumn{5}{|c|}{ trans-1,2-dihexylcyclobutane (high conversion) } \\
\hline & Carbon 1 & Carbon 2 & Carbon 3 & Carbon 4 \\
\hline & 1.00000 & 0.98981 & 0.98958 & 0.97334 \\
\hline & 1.00000 & 0.98609 & 0.98879 & 0.97462 \\
\hline & 1.00000 & 0.98856 & 0.98725 & 0.97602 \\
\hline Average & 1.00000 & 0.98815 & 0.98854 & 0.97466 \\
\hline$\sigma$ & - & 0.00154262 & 0.00096751 & 0.00109447 \\
\hline \multicolumn{5}{|c|}{ trans-1,2-dihexylcyclobutane (low conversion) } \\
\hline & Carbon 1 & Carbon 2 & Carbon 3 & Carbon 4 \\
\hline & 1.00000 & 0.98327 & 0.98972 & 0.97531 \\
\hline & 1.00000 & 0.98448 & 0.98808 & 0.97719 \\
\hline & 1.00000 & 0.98473 & 0.99173 & 0.97563 \\
\hline Average & 1.00000 & 0.98338 & 0.99046 & 0.97561 \\
\hline$\sigma$ & 1.00000 & 0.00143218 & 0.00127426 & 0.00104391 \\
\hline Int/Int ${ }^{\circ}$ & - & 0.995176 & 1.001943 & 1.00097 \\
\hline
\end{tabular}




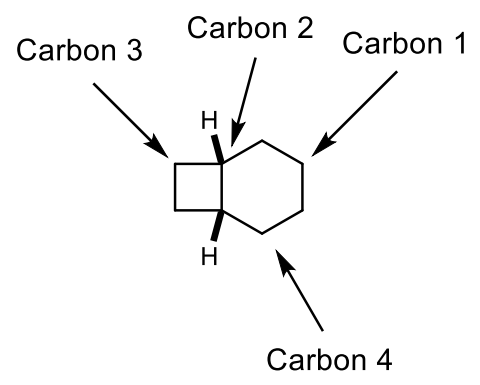

\begin{tabular}{|c|c|c|c|c|}
\hline \multicolumn{5}{|c|}{ cis-bicyclo[4.2.0]octane (high conversion) } \\
\hline & Carbon 1 & Carbon 2 & Carbon 3 & Carbon 4 \\
\hline & 1.00000 & 0.99018 & 0.99372 & 0.98531 \\
\hline & 1.00000 & 0.98986 & 0.99383 & 0.98611 \\
\hline & 1.00000 & 0.98938 & 0.99402 & 0.98564 \\
\hline Average & 1.00000 & 0.98979 & 0.99383 & 0.98578 \\
\hline$\sigma$ & - & 0.00062439 & 0.00040013 & 0.000417031 \\
\hline \multicolumn{5}{|c|}{ cis-bicyclo[4.2.0]octane (low conversion) } \\
\hline & Carbon 1 & Carbon 2 & Carbon 3 & Carbon 4 \\
\hline & 1.00000 & 0.98807 & 0.97307 & 0.98217 \\
\hline & 1.00000 & 0.98843 & 0.97302 & 0.98138 \\
\hline & 1.00000 & 0.98718 & 0.9733 & 0.98261 \\
\hline Average & 1.00000 & 0.98792 & 0.97352 & 0.98206 \\
\hline$\sigma$ & 1.00000 & 0.000942091 & 0.00061818 & 0.000572633 \\
\hline Int/Int ${ }^{\circ}$ & - & 0.998107 & 0.979565 & 0.996221 \\
\hline
\end{tabular}




\section{- Kinetic Measurements}

Procedure for Kinetic Measurements - 1-octene: For each run (repeated twice), in an $\mathrm{N}_{2}$-filled glovebox, GC vials containing magnetic stir-bars were charged with (tric PDI)Fe( $\left.\mathrm{N}_{2}\right)$, cyclooctane, pentane, and 1-octene in that order (see table for amounts). Aliquots $(\sim 1 \mu \mathrm{L})$ were removed from the reaction at regular time intervals and diluted into vials containing $1 \mathrm{~mL}$ of cold $\left(-35^{\circ} \mathrm{C}\right)$ pentane. After completion of the designated reaction time period, the vials were removed from the glovebox and quenched by exposure to air. The aliquots were then analyzed via gas chromatography.

\begin{tabular}{|c|c|c|c|c|}
\hline \multicolumn{5}{|c|}{ Kinetics (1-octene) } \\
\hline Run & 1 -octene & Fe & cyclooctane & pentane \\
\hline 1 & $112 \mu \mathrm{L}(50 \mathrm{M})$ & $8 \mathrm{mg}(1 \mathrm{~mol} \%)$ & $10 \mu \mathrm{L}$ & $10 \mu \mathrm{L}$ \\
\hline 2 & $112 \mu \mathrm{L}(50 \mathrm{M})$ & $16 \mathrm{mg}(2 \mathrm{~mol} \%)$ & $10 \mu \mathrm{L}$ & $10 \mu \mathrm{L}$ \\
\hline 3 & $112 \mu \mathrm{L}(50 \mathrm{M})$ & $32 \mathrm{mg}(4 \mathrm{~mol} \%)$ & $10 \mu \mathrm{L}$ & $10 \mu \mathrm{L}$ \\
\hline 4 & $112 \mu \mathrm{L}(100 \mathrm{M})$ & $4 \mathrm{mg}(0.5 \mathrm{~mol} \%)$ & $10 \mu \mathrm{L}$ & - \\
\hline 5 & $112 \mu \mathrm{L}(25 \mathrm{M})$ & $16 \mathrm{mg}(2 \mathrm{~mol} \%)$ & $10 \mu \mathrm{L}$ & $30 \mu \mathrm{L}$ \\
\hline
\end{tabular}
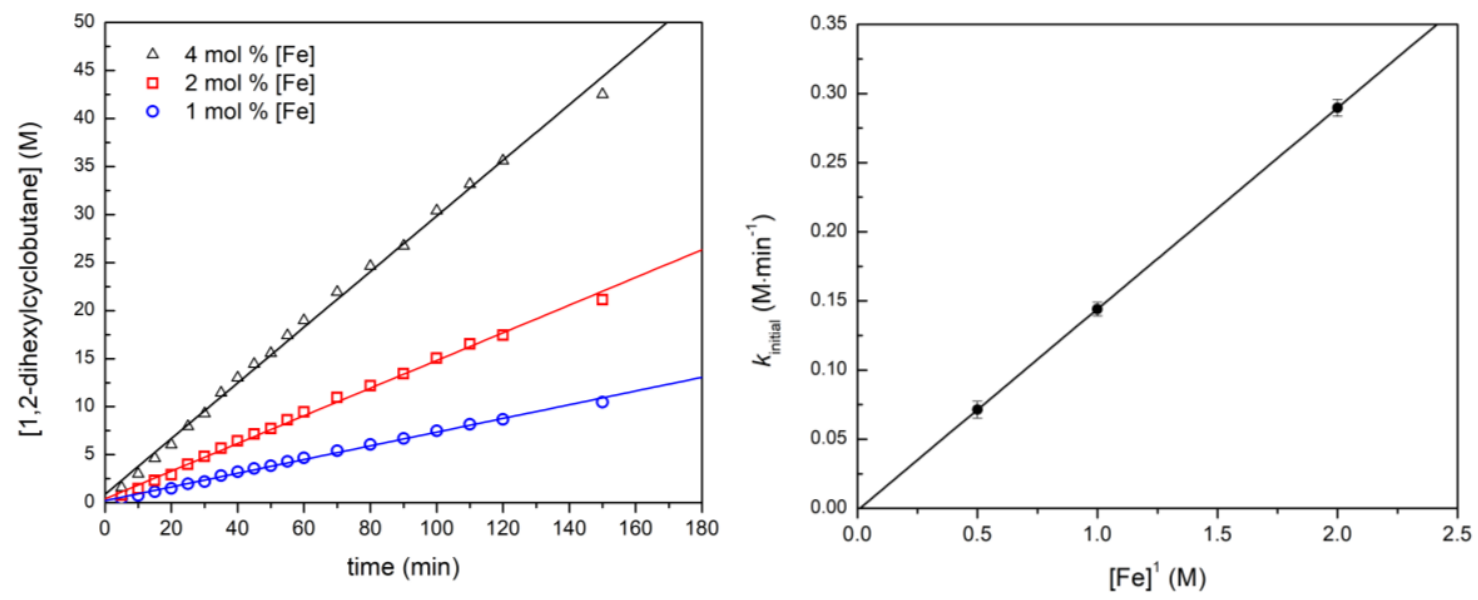

Figure S1. Initial rates plots for determining the order in iron catalyst. Equations for linear regression are as follows: $1 \mathrm{~mol} \% \mathrm{Fe}: y=0.07136 x+0.21418, \mathrm{R}^{2}=0.996 ; 2 \mathrm{~mol} \% \mathrm{Fe}: y=0.14407 x+0.40507$ $R^{2}=0.998 ; 4 \mathrm{~mol} \%$ Fe: $y=0.28977 x+0.88512, R^{2}=0.997$. Right plot: $y=0.14556 x+0.00145$ $R^{2}=0.999$, error bars reflect 1 standard deviation from the mean. 

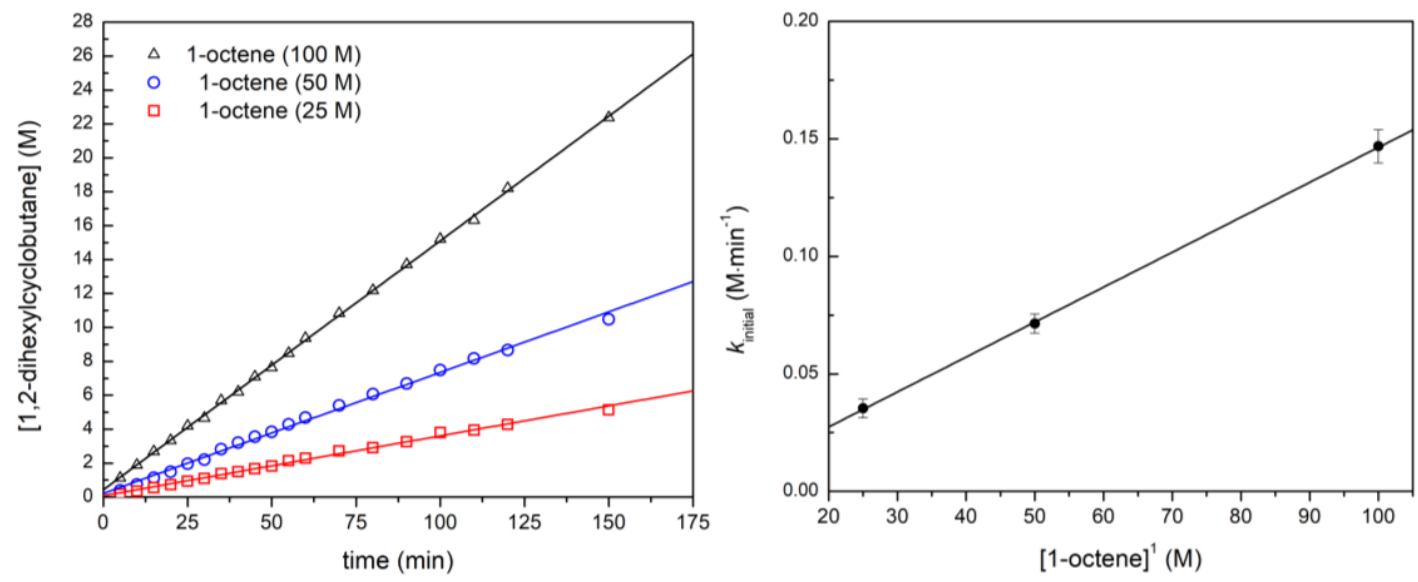

Figure S2. Initial rates plots for determining the order in 1-octene. Equations for linear regression are as follows: 1 -octene $(25 \mathrm{M}): y=0.0353 x+0.793, \mathrm{R}^{2}=0.995 ; 1$-octene $(50 \mathrm{M}): y=0.07136 x+$ 0.21418, $\mathrm{R}^{2}=0.996 ; 1$-octene: $y=0.14682 x+0.43502, \mathrm{R}^{2}=0.999$. Right plot: $y=0.00149 x-$ $0.00223, R^{2}=0.999$, error bars reflect 1 standard deviation from the mean.

Procedure for Kinetic Measurements - propylene: For each run (repeated twice), in an $\mathrm{N}_{2}$-filled glovebox, a J. Young NMR tube was charged with (ric PDI)Fe $\left(\mathrm{N}_{2}\right)$, benzene- $d_{6}$, and mesitylene (see table for amounts), sealed, and then brought outside of the glovebox. On a Schlenk line, the tube was frozen in liquid nitrogen $\left(-196^{\circ} \mathrm{C}\right)$ and the solution degassed via three freeze-pump-thaw cycles. Propylene $(11.1 \mathrm{~cm} \mathrm{Hg}, 0.600 \mathrm{mmol})$ was then introduced into the tube via a calibrated gas bulb (100.1 mL). For runs with $1 \mathrm{~atm}$ of $\mathrm{N}_{2}$, the entire tube was immersed in liquid nitrogen and $19.8 \mathrm{~cm}$ $\mathrm{Hg}$ of $\mathrm{N}_{2}$ was introduced (with a tube volume of $2.7 \mathrm{~mL}$, this will equal $1 \mathrm{~atm} \mathrm{~N}_{2}$ at $23^{\circ} \mathrm{C}$ ). The tube was then sealed and removed from the Schlenk line. For runs with 0 atm of $\mathrm{N}_{2}$, the tube was simply sealed and removed from the Schlenk line. While still frozen in liquid nitrogen, the tube was brought to the NMR instrument and warmed between gloved hands until the solution just thawed. The tube was then inverted 3 times, fitted with an NMR spinner, and placed in the instrument. An automated NMR program set for data collection at regular intervals was then initiated within 1 minute of inverting the tube. \% yield for each time interval spectrum was calculated relative to the internal standard. 


\begin{tabular}{|c|c|c|c|c|c|}
\hline \multicolumn{5}{|c|}{ Kinetics (propylene) } \\
\hline Run & propylene & Fe & mesitylene & benzene- $d_{6}$ & $\mathrm{~N}_{2}$ \\
\hline 1 & $11.1 \mathrm{~cm} \mathrm{Hg}(1.2 \mathrm{M})$ & $7 \mathrm{mg}(1 \mathrm{~mol} \%)$ & $27.8 \mu \mathrm{L}$ & $500 \mu \mathrm{L}$ & $1 \mathrm{~atm}$ \\
\hline 2 & $11.1 \mathrm{~cm} \mathrm{Hg}(1.2 \mathrm{M})$ & $7 \mathrm{mg}(1 \mathrm{~mol} \%)$ & $27.8 \mu \mathrm{L}$ & $500 \mu \mathrm{L}$ & $0 \mathrm{~atm}$ \\
\hline
\end{tabular}
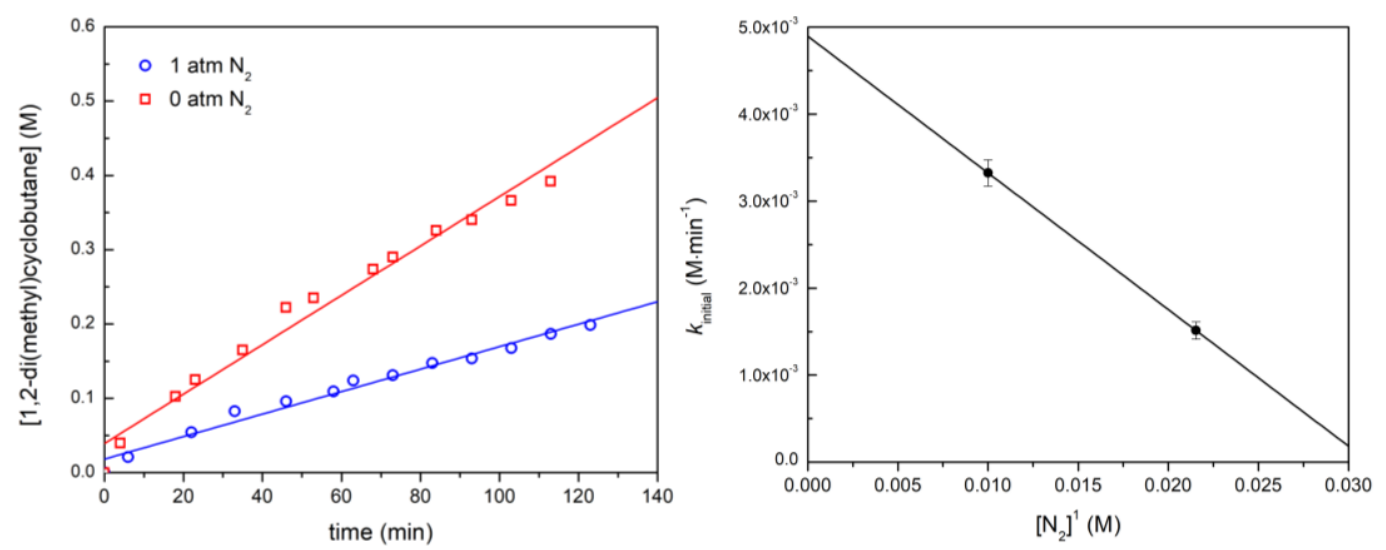

Figure S3. Initial rates methods for determining the order in $\mathrm{N}_{2}$. Equations for linear regression are as follows: $1 \mathrm{~atm} \mathrm{~N}_{2}: y=0.00151 x+0.01822, \mathrm{R}^{2}=0.987 ; 0 \mathrm{~atm} \mathrm{~N}_{2}: y=0.00332 x+0.03915, \mathrm{R}^{2}=$ 0.982. Right plot: $y=-0.15708 x-0.00489, R^{2}=0.999$, error bars reflect 1 standard deviation from the mean.

\section{Determining the Amount of $\mathrm{N}_{2}$ in Each Run:}

- 1 atm $\mathrm{N}_{2}=4.34 \times 10^{-4}$ mole fraction of $\mathrm{N}_{2}$ in benzene ---> $2.92 \times 10^{-6} \mathbf{m o l ~} \mathrm{N}_{2}$ in $500 \mu L$ of benzene (Solubility Data Series: Nitrogen and Air" Battino, R. Ed. Pergamon Press Inc., Elmsford, NY, 1982.) - $\mathrm{Fe}\left(\mathrm{N}_{2}\right)$ total added $=0.006 \mathrm{mmol}--->\mathrm{Fe}\left(\mathrm{N}_{2}\right)_{2}: \mathrm{Fe}\left(\mathrm{N}_{2}\right)$ in benzene $=2.1: 1--->10 \times 10^{-6} \mathrm{~mol} \mathrm{~N}_{\mathbf{2}}$ total - 1 atm $\mathrm{N}_{2}=12.92 \times 10^{-6} \mathrm{~mol} \mathrm{~N}_{2}$ vs. 0 atm $\mathrm{N}_{2}=6.0 \times 10^{-6} \mathrm{~mol} \mathrm{~N}_{2}$

- Ratio of 2.15:1, and the initial rates of 0 vs 1 atm $N_{2}$ is 2.19:1.

Procedure for Kinetic Measurements - 1,7-octadiene: For each run (repeated twice), in an $\mathrm{N}_{2-}$ filled glovebox, a J. Young NMR tube was charged with (tricPDI)Fe( $\left.\mathrm{N}_{2}\right)$, hexamethyldisiloxane, and benzene- $d_{6}$ (see table for amounts). The tube was then frozen in a cold well $\left(-196{ }^{\circ} \mathrm{C}\right)$ and charged with 1,7-octadiene (see table for amounts), sealed and then quickly removed from the glovebox and 
placed in a dewar filled with liquid nitrogen. The tube was then brought to the NMR instrument and warmed between gloved hands until the solution just thawed. The tube was then inverted 3 times, fitted with an NMR spinner, and placed in the instrument. An automated NMR program set for data collection at regular intervals was then initiated within 1 minute of inverting the tube. \% yield for each time interval spectrum was calculated relative to the internal standard. For the runs with 0 atm $\mathrm{N}_{2}$, a tube with just (tricPDI)Fe( $\left.\mathrm{N}_{2}\right)$ and a tube containing hexamethyldisiloxane, 1,7-octadiene, and benzene- $d_{6}$ were charged in the glovebox and then removed. The solution-containing tube was degassed via three freeze-pump-thaw cycles and then transferred to the catalyst-containing J. Young tube via a tube-to-tube vacuum distillation.

\begin{tabular}{|c|c|c|c|c|c|}
\hline \multicolumn{7}{|c|}{ Kinetics $(1,7$-octadiene $)$} \\
\hline Run & 1,7 -octadiene & Fe & $\left(\mathrm{Me}_{3} \mathrm{Si}\right)_{2} \mathrm{O}$ & benzene- $d_{6}$ & $\mathrm{~N}_{2}$ \\
\hline 1 & $60 \mu \mathrm{L}(0.86 \mathrm{M})$ & $16 \mathrm{mg}(5 \mathrm{~mol} \%)$ & $10 \mu \mathrm{L}$ & $472 \mu \mathrm{L}$ & $1 \mathrm{~atm}$ \\
\hline 2 & $60 \mu \mathrm{L}(0.86 \mathrm{M})$ & $8 \mathrm{mg}(2.5 \mathrm{~mol} \%)$ & $10 \mu \mathrm{L}$ & $472 \mu \mathrm{L}$ & $1 \mathrm{~atm}$ \\
\hline 3 & $60 \mu \mathrm{L}(0.86 \mathrm{M})$ & $33 \mathrm{mg}(10 \mathrm{~mol} \%)$ & $10 \mu \mathrm{L}$ & $472 \mu \mathrm{L}$ & $1 \mathrm{~atm}$ \\
\hline 4 & $30 \mu \mathrm{L}(0.43 \mathrm{M})$ & $16 \mathrm{mg}(5 \mathrm{~mol} \%)$ & $10 \mu \mathrm{L}$ & $472 \mu \mathrm{L}$ & $1 \mathrm{~atm}$ \\
\hline 5 & $120 \mu \mathrm{L}(1.72 \mathrm{M})$ & $16 \mathrm{mg}(5 \mathrm{~mol} \%)$ & $10 \mu \mathrm{L}$ & $472 \mu \mathrm{L}$ & $1 \mathrm{~atm}$ \\
\hline 6 & $60 \mu \mathrm{L}(0.86 \mathrm{M})$ & $16 \mathrm{mg}(5 \mathrm{~mol} \%)$ & $10 \mu \mathrm{L}$ & $472 \mu \mathrm{L}$ & $0 \mathrm{~atm}$ \\
\hline
\end{tabular}
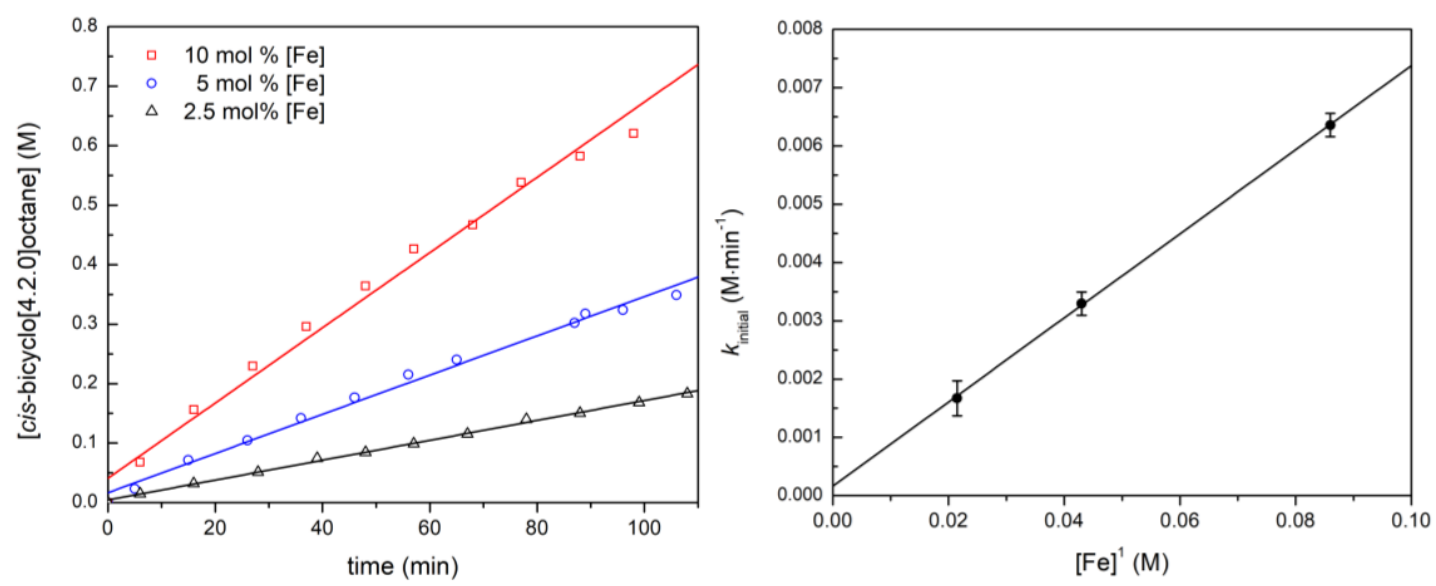

Figure S4. Initial rates plots for determining the order in iron catalyst. Equations for linear regression are as follows: $2.5 \mathrm{~mol} \% \mathrm{Fe}: y=0.00167 x+0.00447, \mathrm{R}^{2}=0.998 ; 5 \mathrm{~mol} \% \mathrm{Fe}: y=0.0032 x+$ 
$0.02017, R^{2}=0.994 ; 10$ mol \% Fe: $y=0.00632 x+0.0493, R^{2}=0.989$. Right plot: $y=0.07215 x+$ $0.000164, R^{2}=0.999$, error bars reflect 1 standard deviation from the mean.
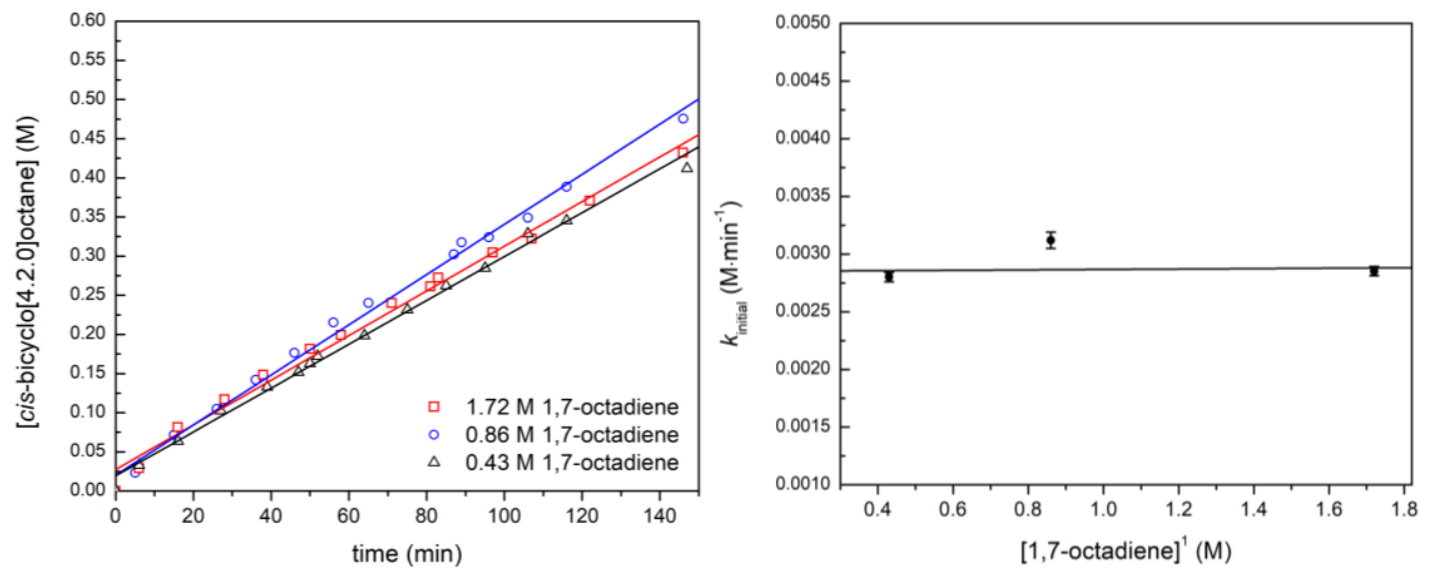

Figure S5. Initial rates plots for determining the order in 1,7-octadiene. Equations for linear regression are as follows: 1,7 -ocadiene $(0.86 \mathrm{M}): y=0.0032 x+0.02017, \mathrm{R}^{2}=0.994 ; 1,7$-octadiene $(0.43 \mathrm{M}): y=0.0028 x+0.0194, \mathrm{R}^{2}=0.994 ; 1,7$-octadiene $(1.72 \mathrm{M}): y=0.00285 x+0.02742, \mathrm{R}^{2}=$ 0.990. Right plot: $y=0.0000181 x+0.000285, R^{2}=0.982$, error bars reflect 1 standard deviation from the mean.
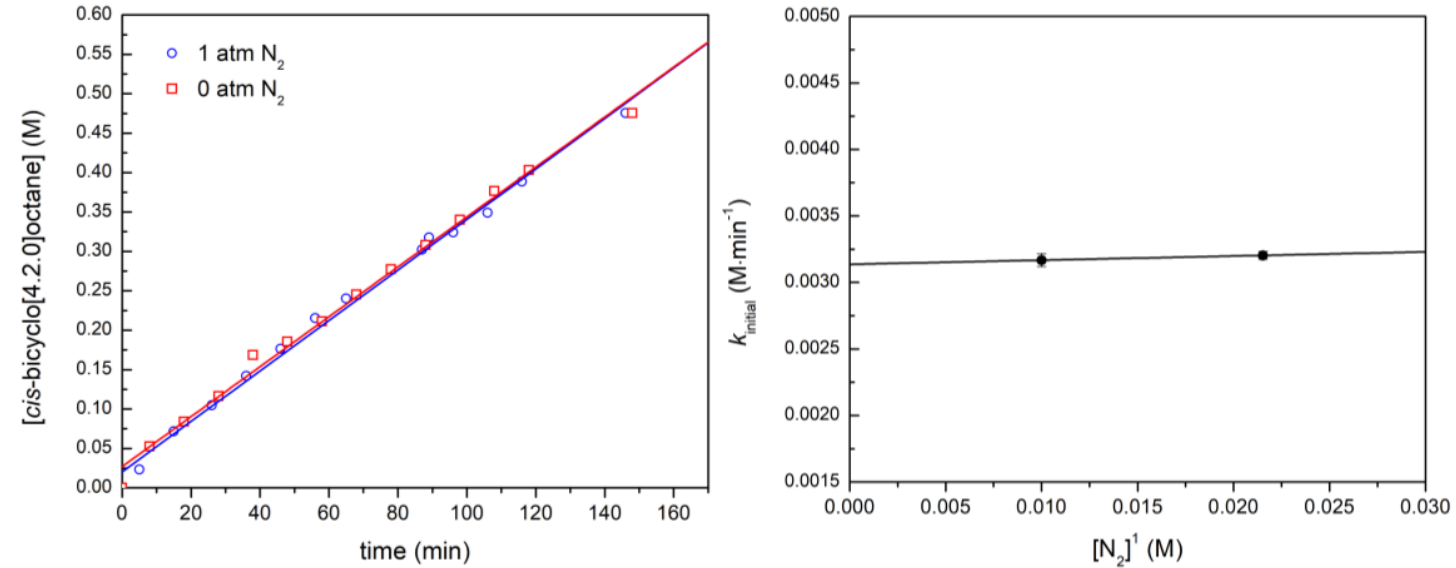

Figure S6. Initial rates plots for determining the order in $\mathrm{N}_{2}$. Equations for linear regression are as follows: 1 atm $\mathrm{N}_{2}: y=0.0032+0.02017, \mathrm{R}^{2}=0.994 ; 0$ atm $\mathrm{N}_{2}: y=0.00317+0.02706$. Right plot: $y$ $=0.00031 x+0.00314, R^{2}=0.999$, error bars reflect 1 standard deviation from the mean. 


\section{NMR Spectra}
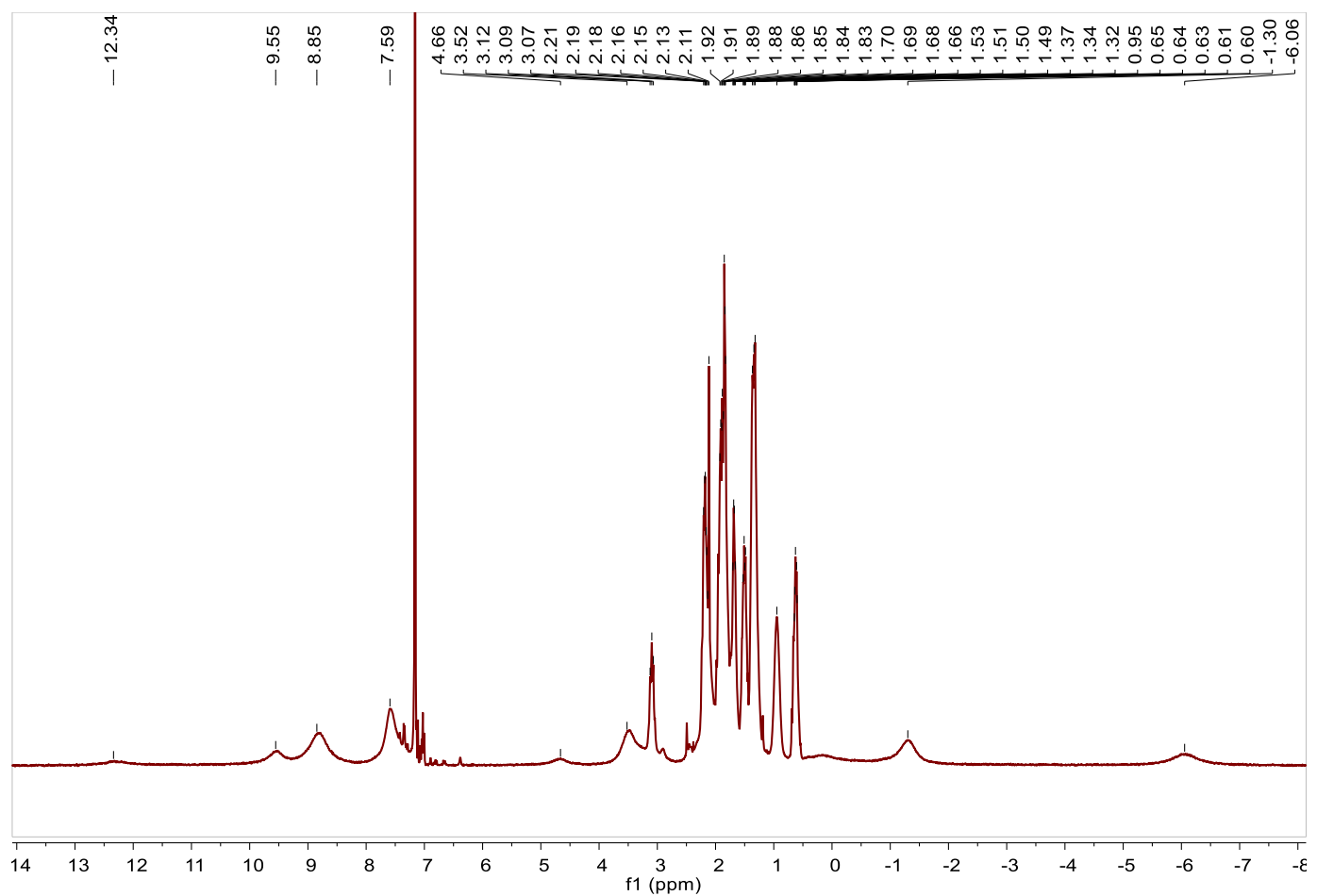

Figure S7. ${ }^{1} \mathrm{H}$ NMR spectrum $\left(300 \mathrm{MHz}\right.$, benzene- $\left.d_{6}, 23^{\circ} \mathrm{C}\right)$ of (tric PDI)Fe( $\left.\mathrm{N}_{2}\right), 2.1: 1 \mathrm{mixture}$ of bis to mono dinitrogen species. Contains toluene.

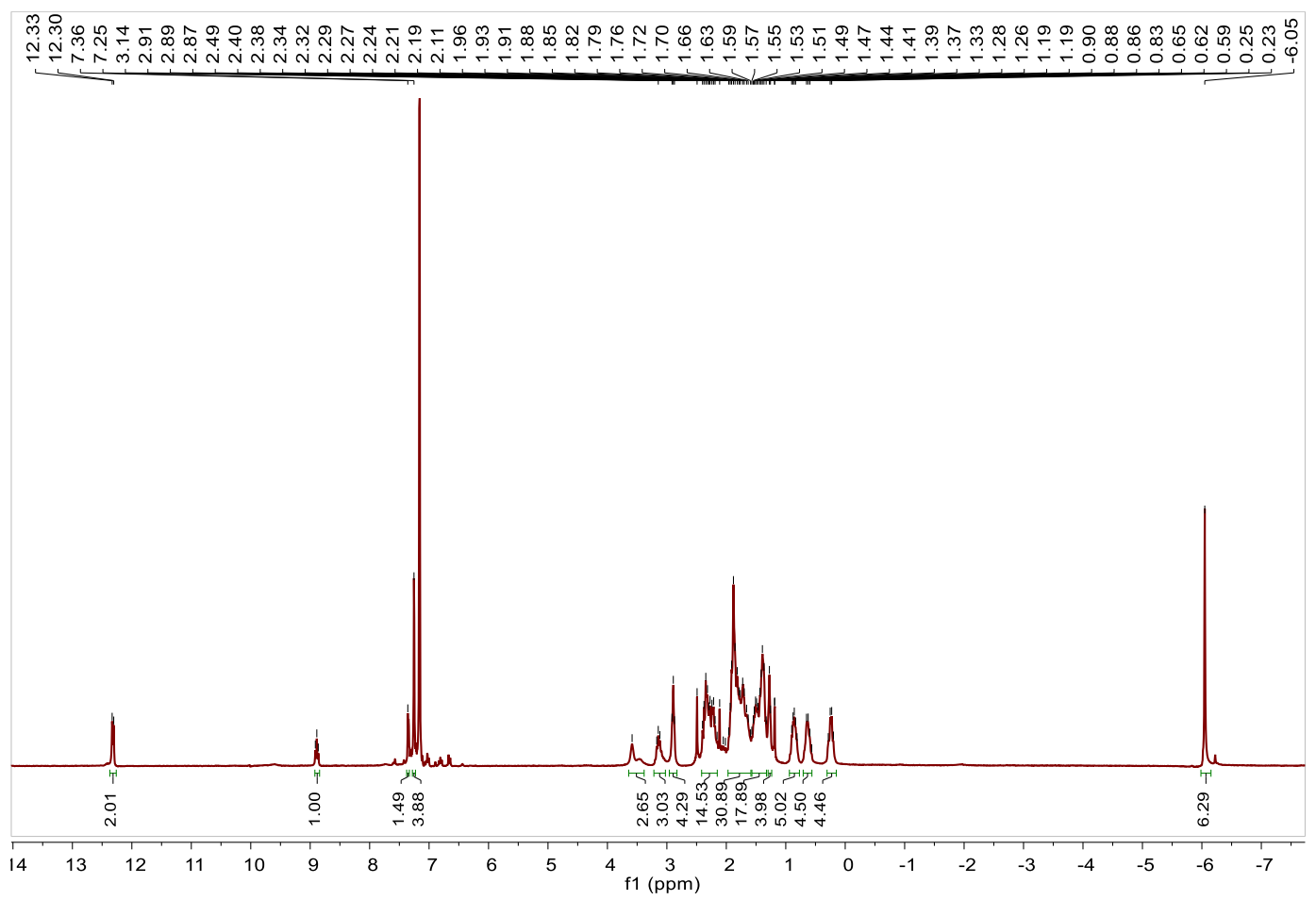

Figure S8. ${ }^{1} \mathrm{H}$ NMR spectrum $\left(300 \mathrm{MHz}\right.$, benzene- $\left.\boldsymbol{d}_{6}, \mathbf{2 3}{ }^{\circ} \mathrm{C}\right)$ of (tric PDI)Fe( $\left.\mathrm{N}_{2}\right)$ at 0 atm $\mathrm{N}_{2}$. 

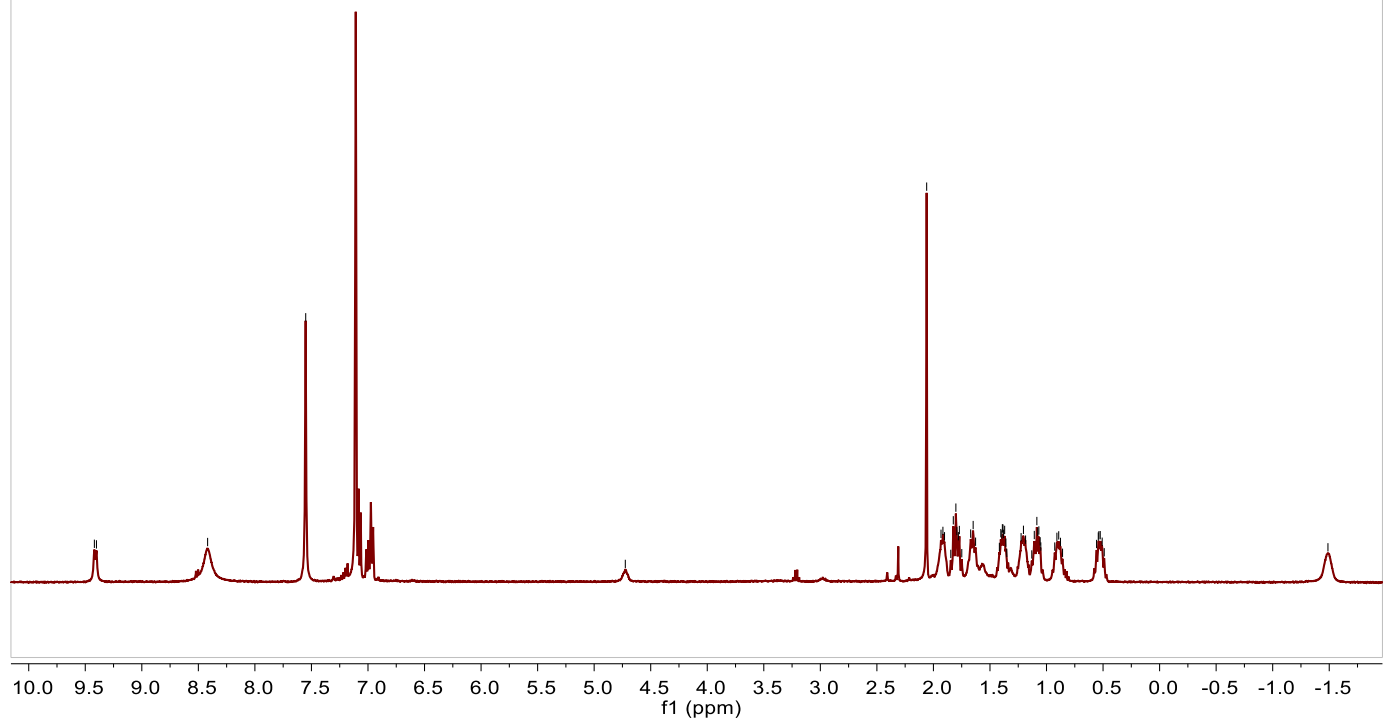

Figure S9. ${ }^{1} \mathrm{H}$ NMR spectrum $\left(400 \mathrm{MHz}\right.$, benzene- $\left.\boldsymbol{d}_{6}, 23^{\circ} \mathrm{C}\right)$ of $\left({ }^{\text {dic }} \mathrm{PDI}\right) \mathrm{Fe}\left(\mathrm{N}_{2}\right)_{2}$.

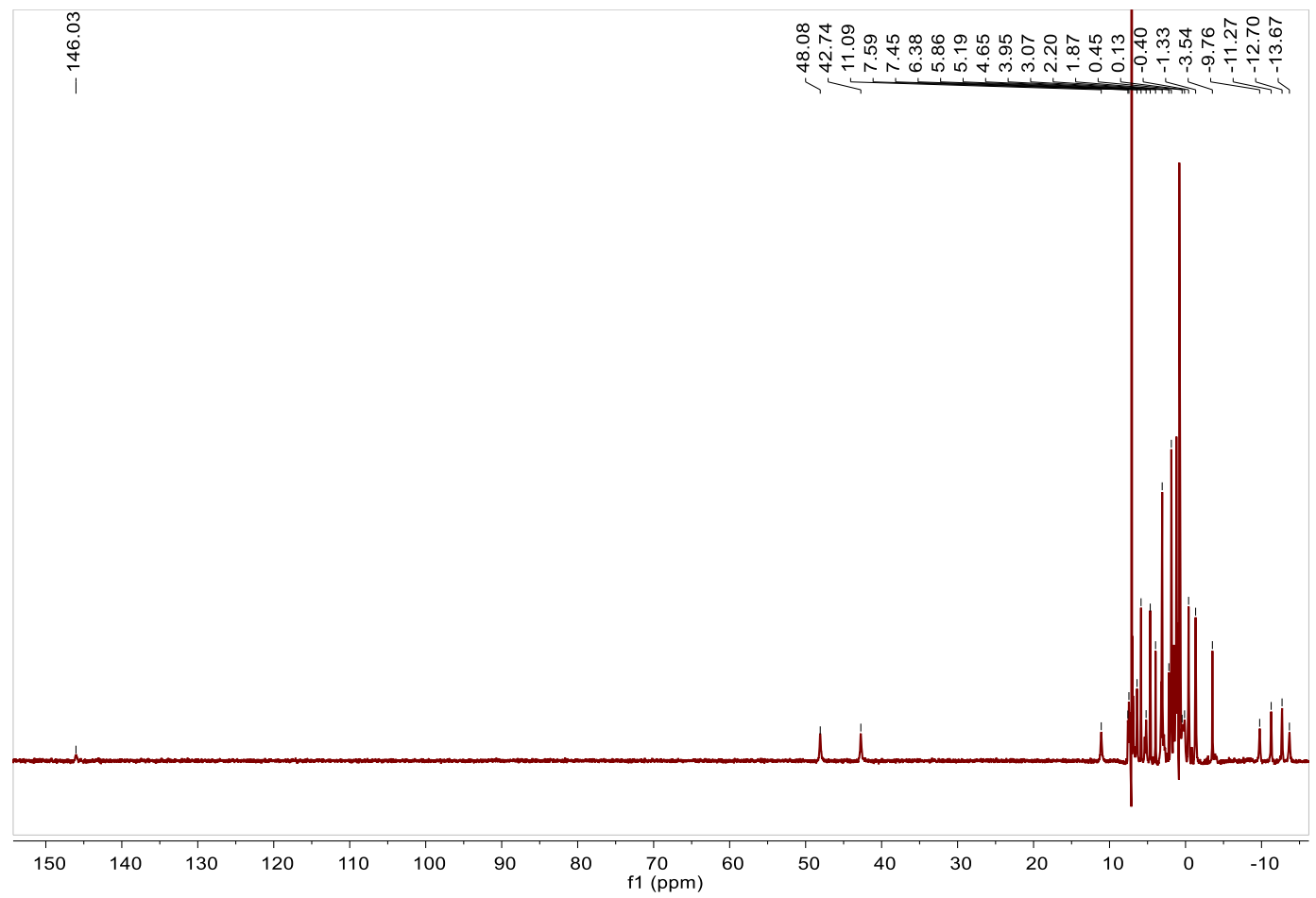

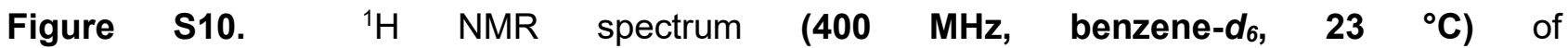
(Pr(TB)PDI)Fe(bimetallacyclo[4.3.0]nonane. 


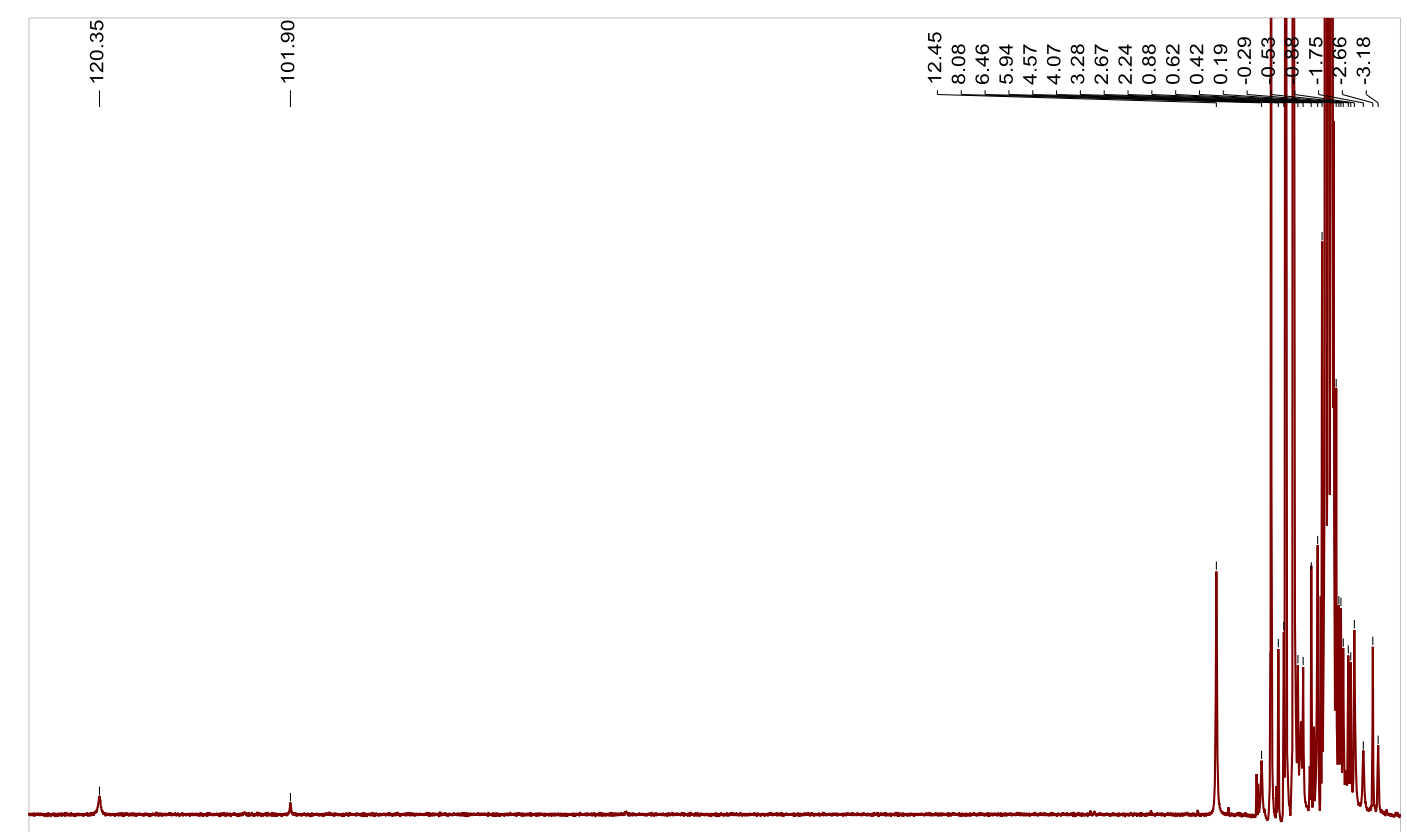

$\begin{array}{llllllllllllllllllllllllllllllllllll}125 & 120 & 115 & 110 & 105 & 100 & 95 & 90 & 85 & 80 & 75 & 70 & 65 & 60 & 55 & 50 & 45 & 40 & 35 & 30 & 25 & 20 & 15 & 10 & 5 & 0 & -1\end{array}$

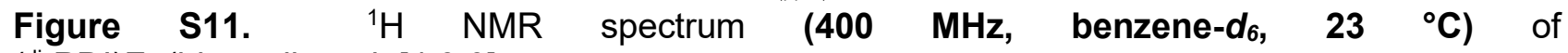
(dicPDI)Fe(bimetallacyclo[4.3.0]nonane.
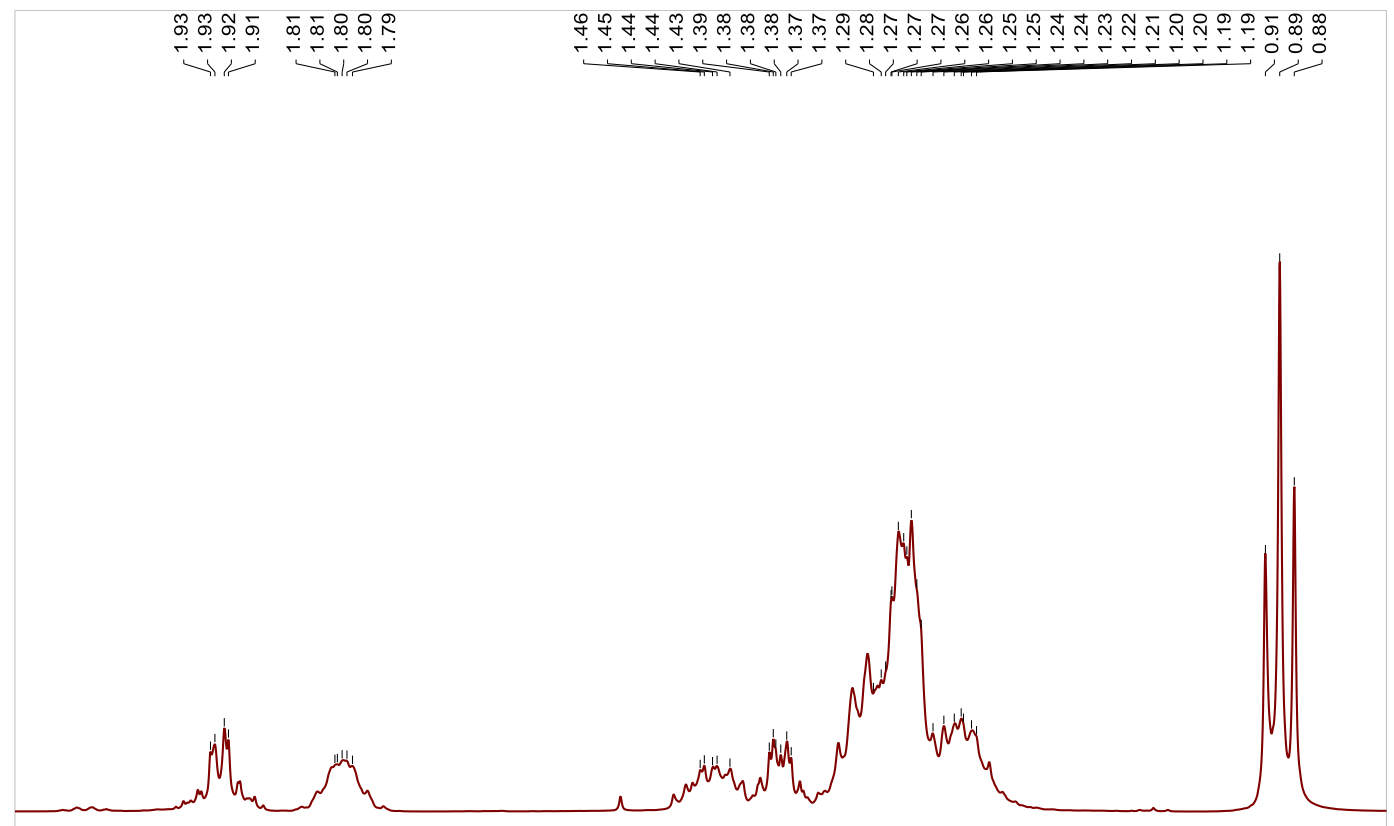

$\begin{array}{lllllllllllllllllllllllllllllllllllllllll}2.10 & 2.05 & 2.00 & 1.95 & 1.90 & 1.85 & 1.80 & 1.75 & 1.70 & 1.65 & 1.60 & 1.55 & 1.50 & 1.45 & 1.40 & 1.35 & 1.30 & 1.25 & 1.20 & 1.15 & 1.10 & 1.05 & 1.00 & 0.95 & 0.90 & 0.85 & 0.8\end{array}$

Figure S12. ${ }^{1} \mathrm{H}$ NMR spectrum (500 MHz, chloroform- $d_{1}, 23^{\circ} \mathrm{C}$ ) of trans-1,2-dihexylcyclobutane 


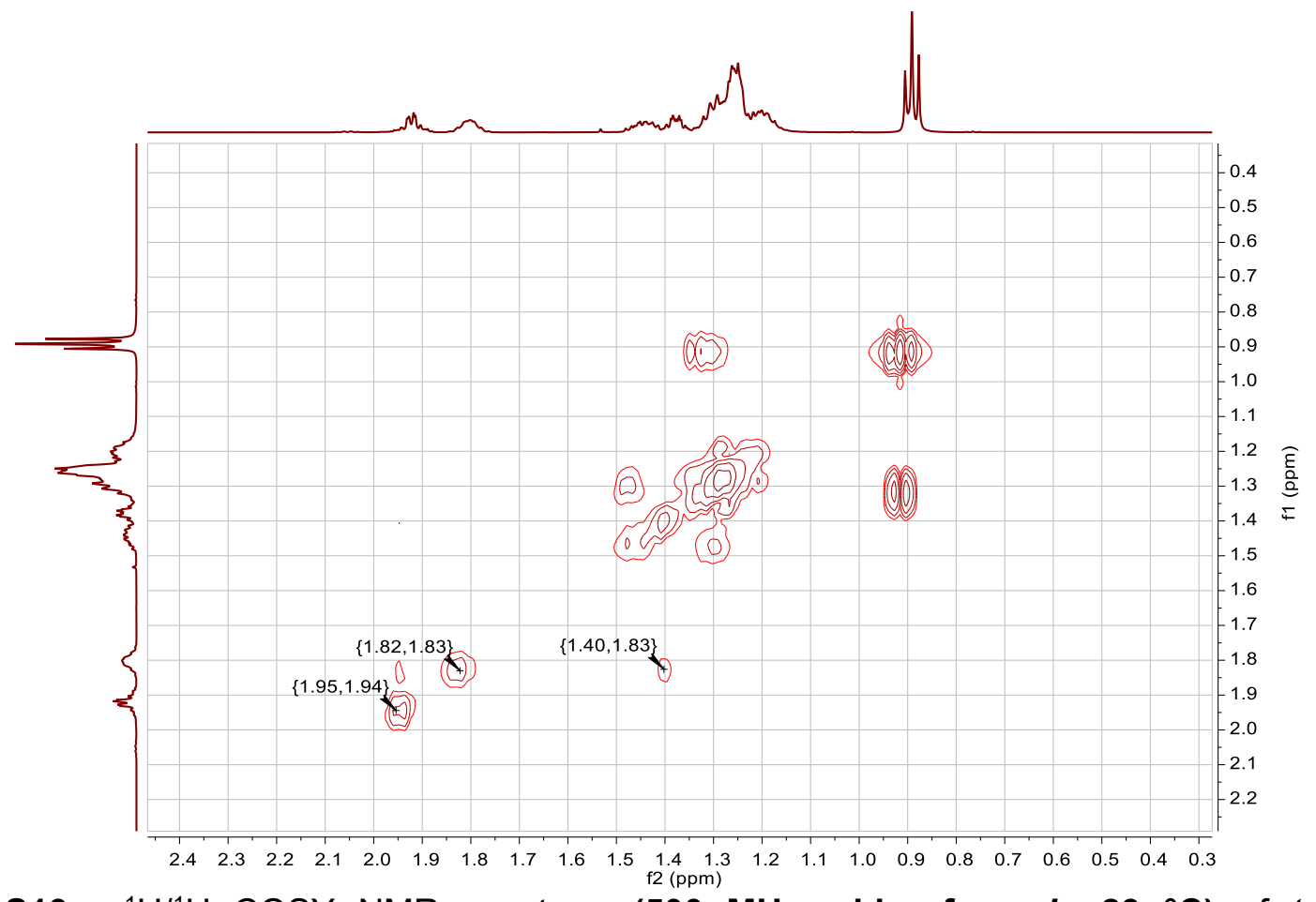

Figure S13. ${ }^{1} \mathrm{H} /{ }^{1} \mathrm{H}$ COSY NMR spectrum (500 MHz, chloroform-d, $\left.23{ }^{\circ} \mathrm{C}\right)$ of trans-1,2dihexylcyclobutane

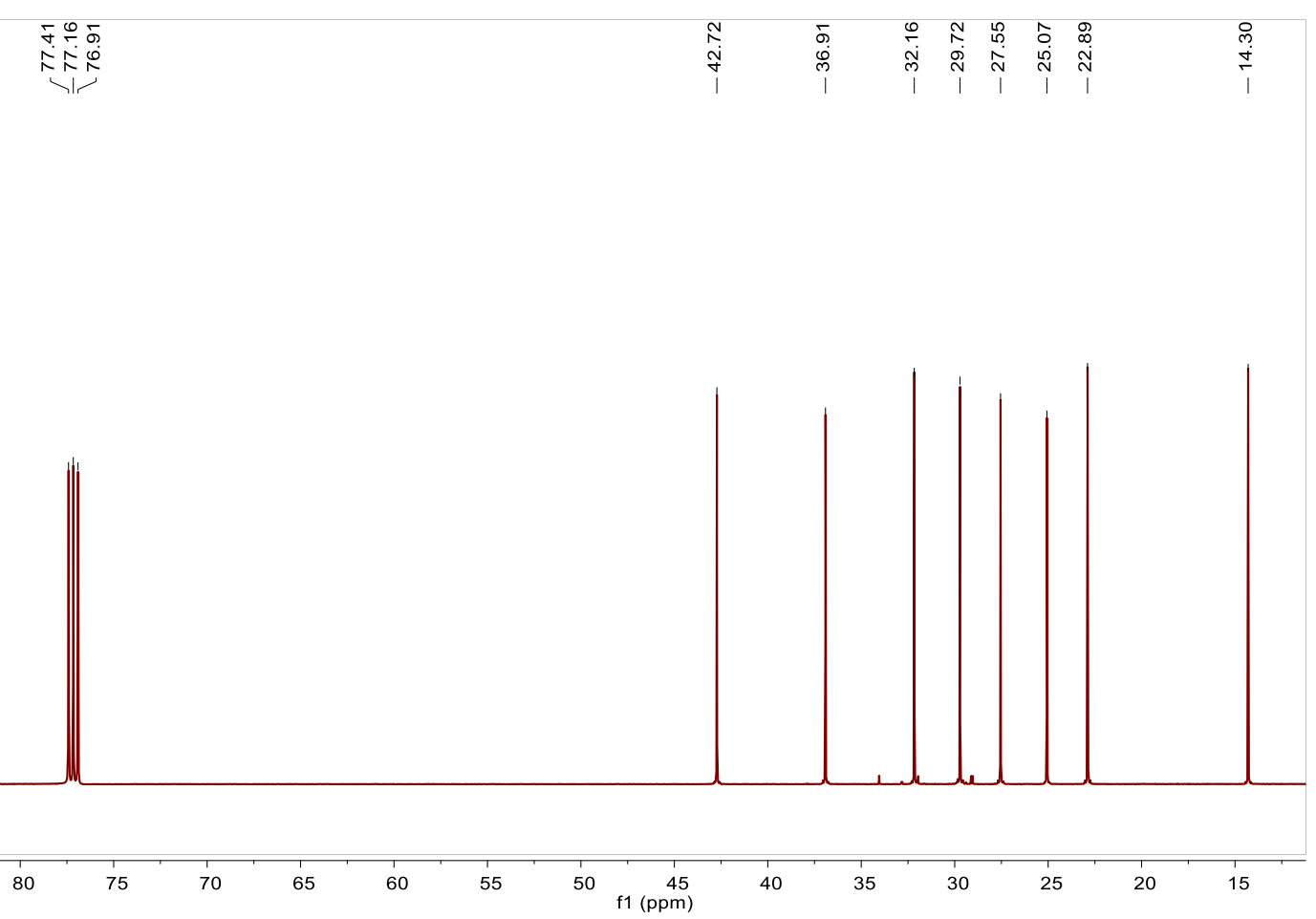

Figure S14. ${ }^{13} \mathrm{C}$ NMR spectrum (128 MHz, chloroform- $\boldsymbol{d}_{\mathbf{1}}, 23^{\circ} \mathrm{C}$ ) of trans-1,2-dihexylcyclobutane 


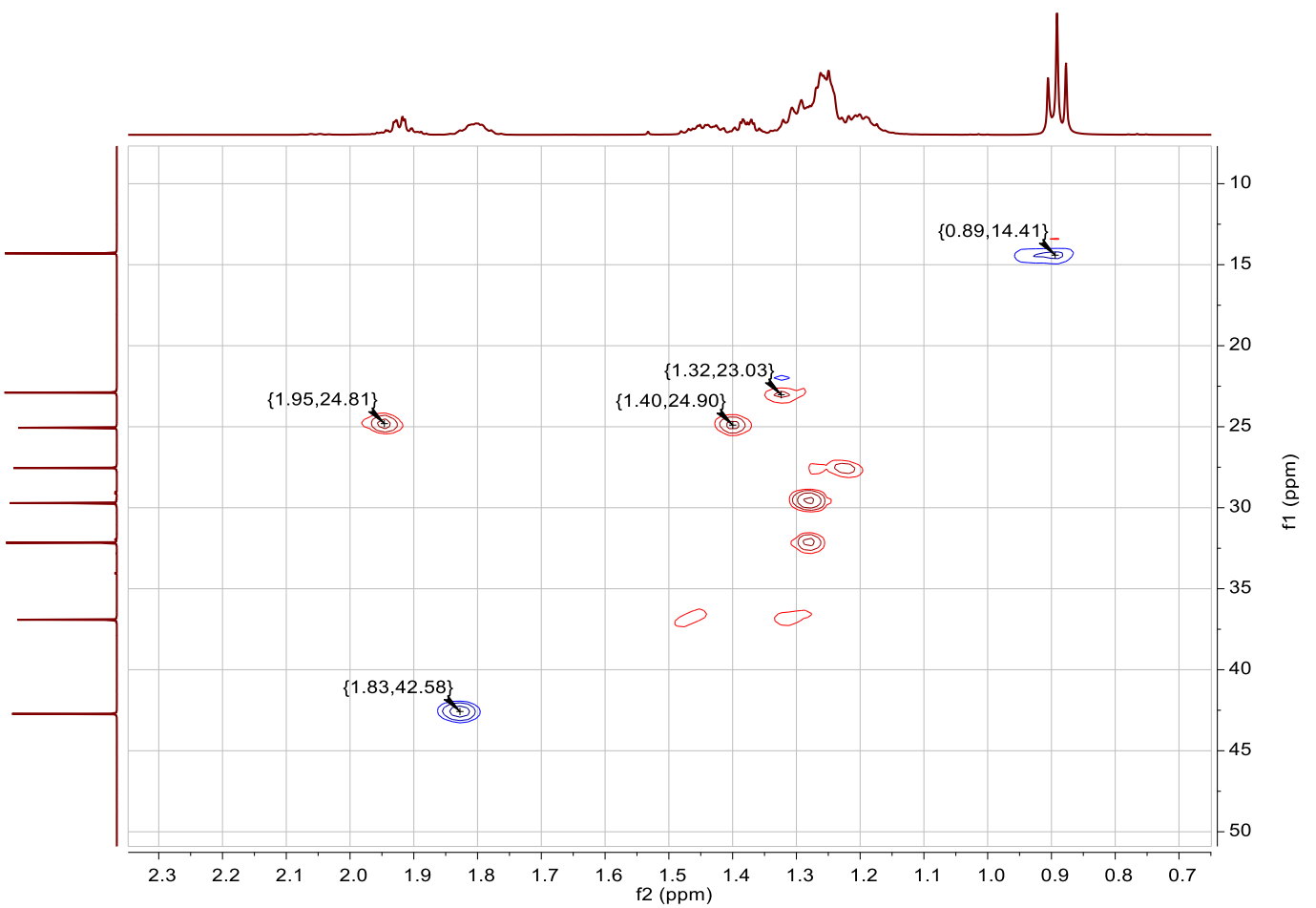

Figure S15. ${ }^{1} \mathrm{H} /{ }^{13} \mathrm{C}$ HSQC NMR spectrum (128 $\mathbf{~ M H z}$, chloroform- $d_{1}, 23{ }^{\circ} \mathrm{C}$ ) of trans-1,2dihexylcyclobutane

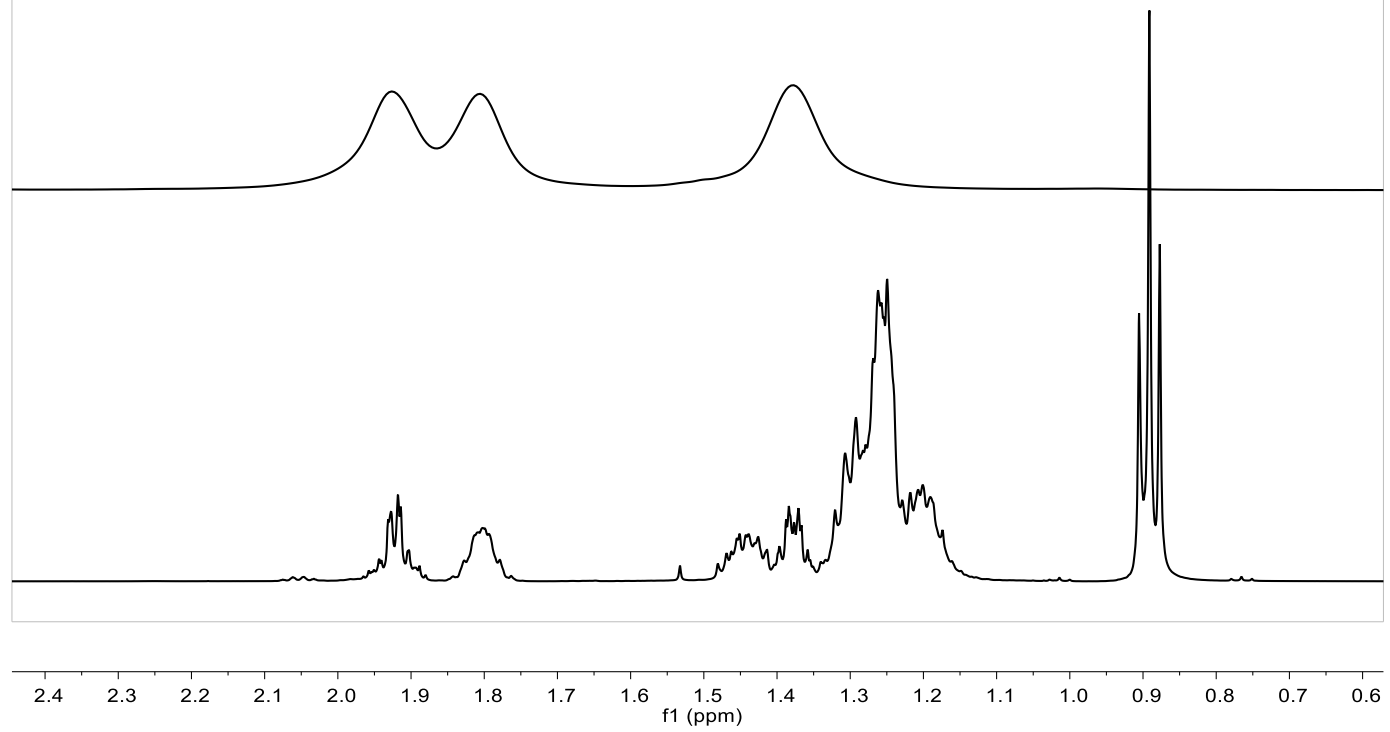

Figure S16. Top - ${ }^{2} \mathrm{H}$ NMR spectrum (77 MHz, chloroform, $23^{\circ} \mathrm{C}$ ) of trans-1,2-dihexylcyclobutane$d_{n}$. Bottom, reference spectrum of protio version. 


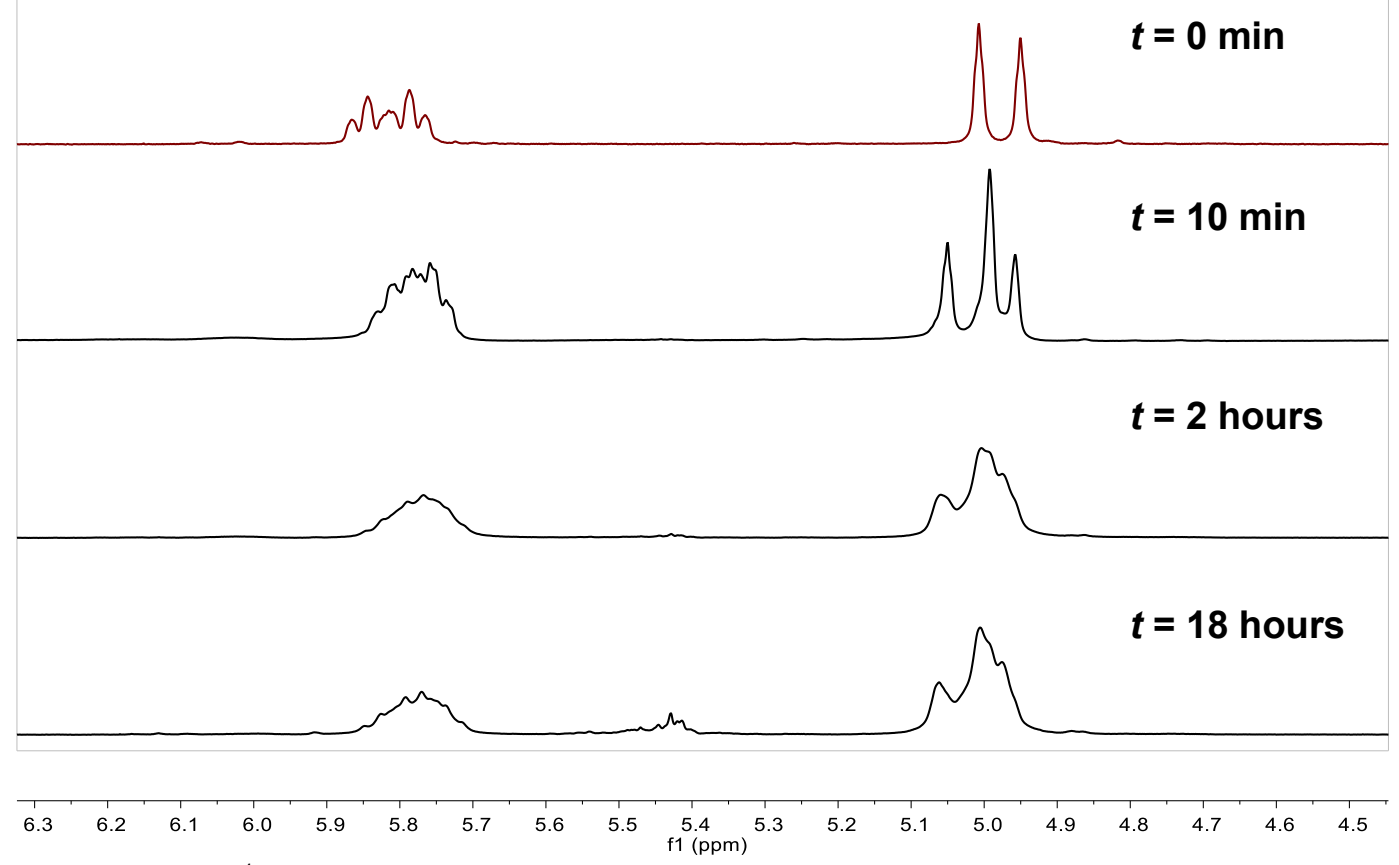

Figure S17. Stacked ${ }^{1} \mathrm{H}$ NMR spectra $\left(300 \mathrm{MHz}\right.$, benzene- $\left.\boldsymbol{d}_{6}, 23^{\circ} \mathrm{C}\right)$ from Scheme 9.

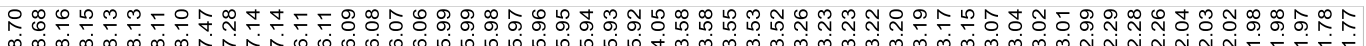

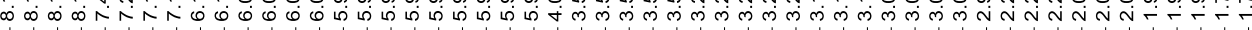

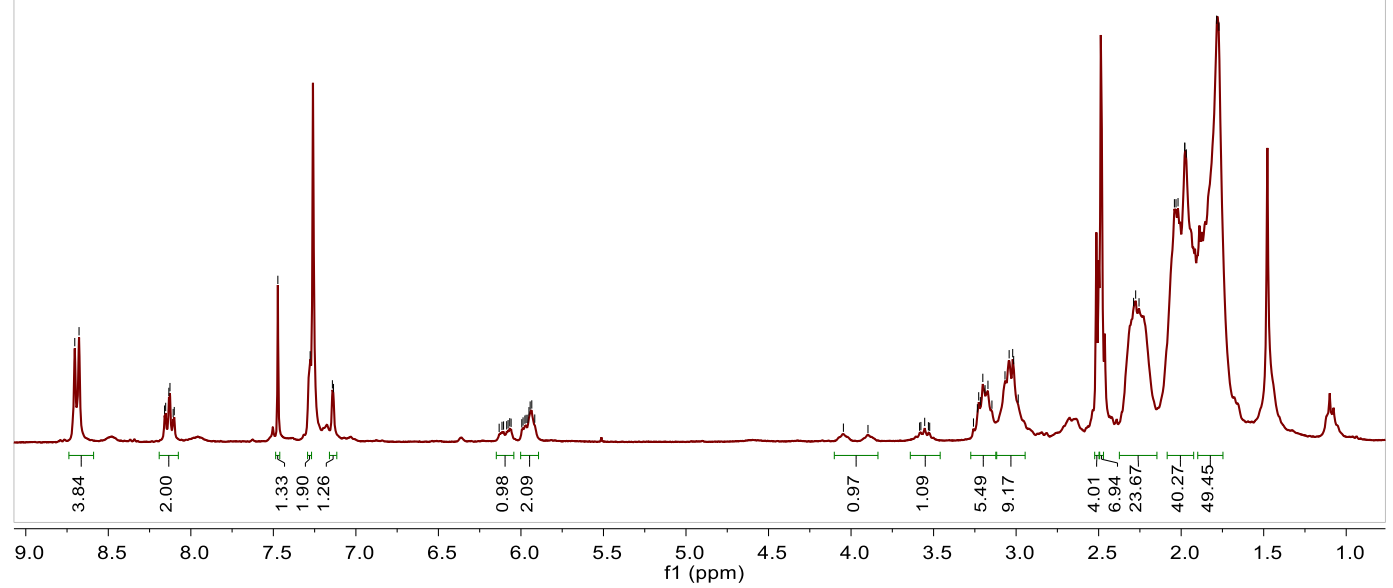

Figure S18. ${ }^{1} \mathrm{H}$ NMR spectrum $\left(300 \mathrm{MHz}\right.$, benzene- $d_{6}, 23^{\circ} \mathrm{C}$ ) of (dehydro-tric PDI), contains pentane. 


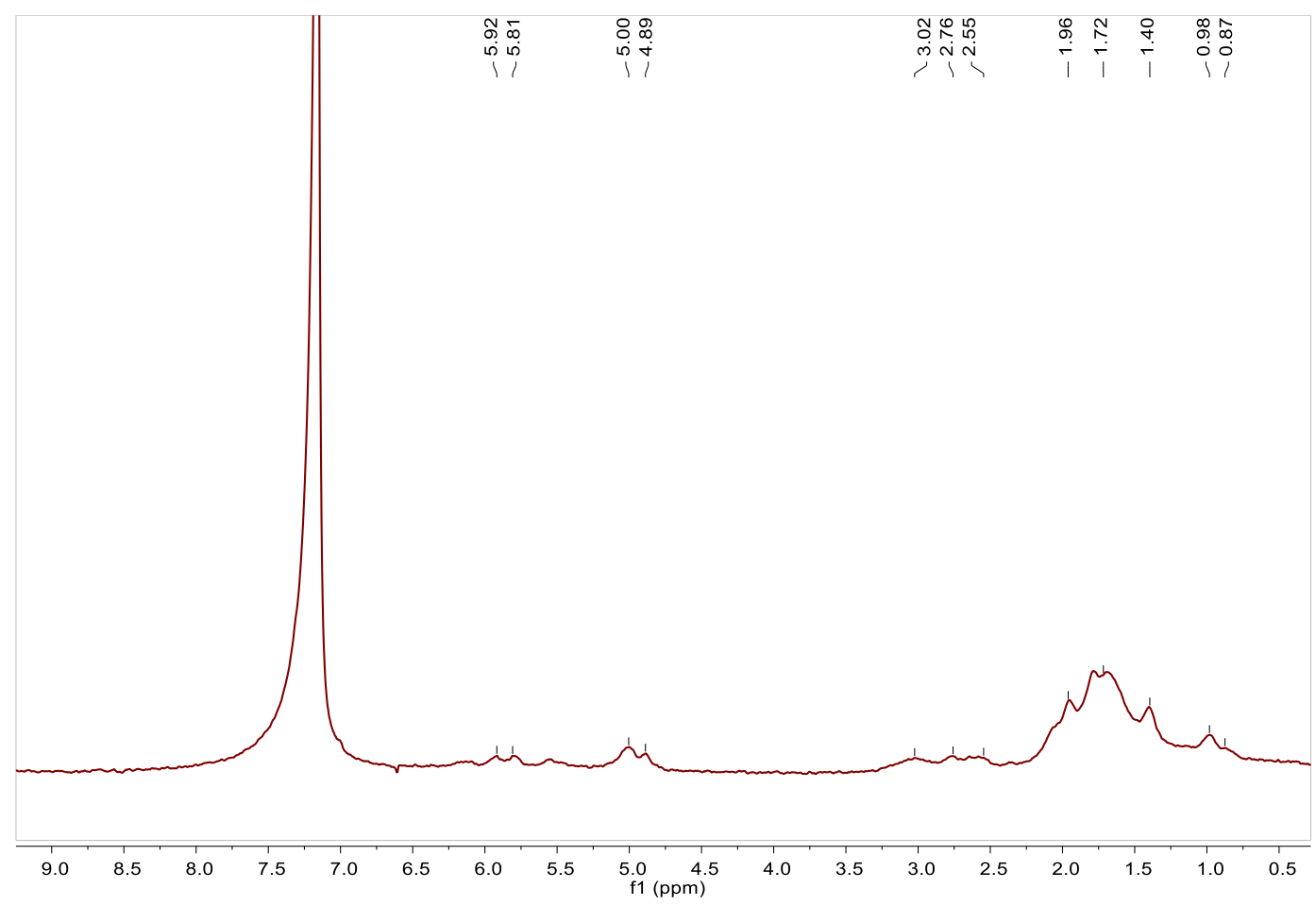

Figure S19. ${ }^{2} \mathrm{H}$ NMR spectrum $\left(77 \mathrm{MHz}\right.$, benzene, $23^{\circ} \mathrm{C}$ ) of (dehydro- ${ }^{\text {tric }}$ PDI).

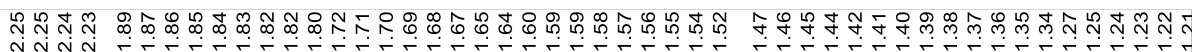

(1)
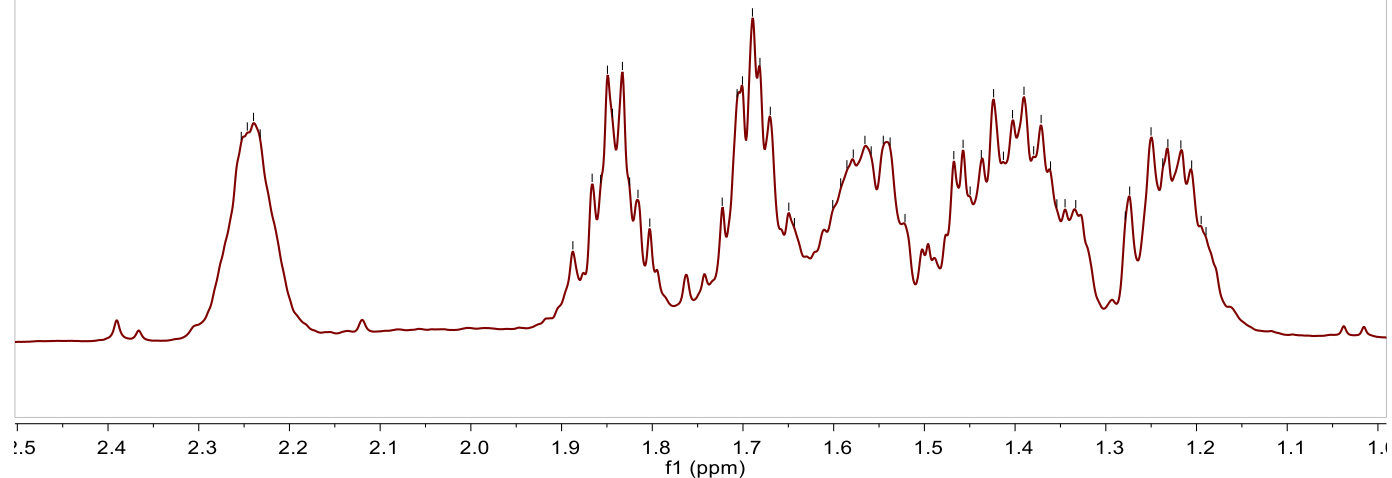

Figure S20. ${ }^{1} \mathrm{H}$ NMR spectrum $\left(300 \mathrm{MHz}\right.$, benzene- $\left.d_{6}, 23^{\circ} \mathrm{C}\right)$ of cis-bicyclo[4.2.0]octane. 


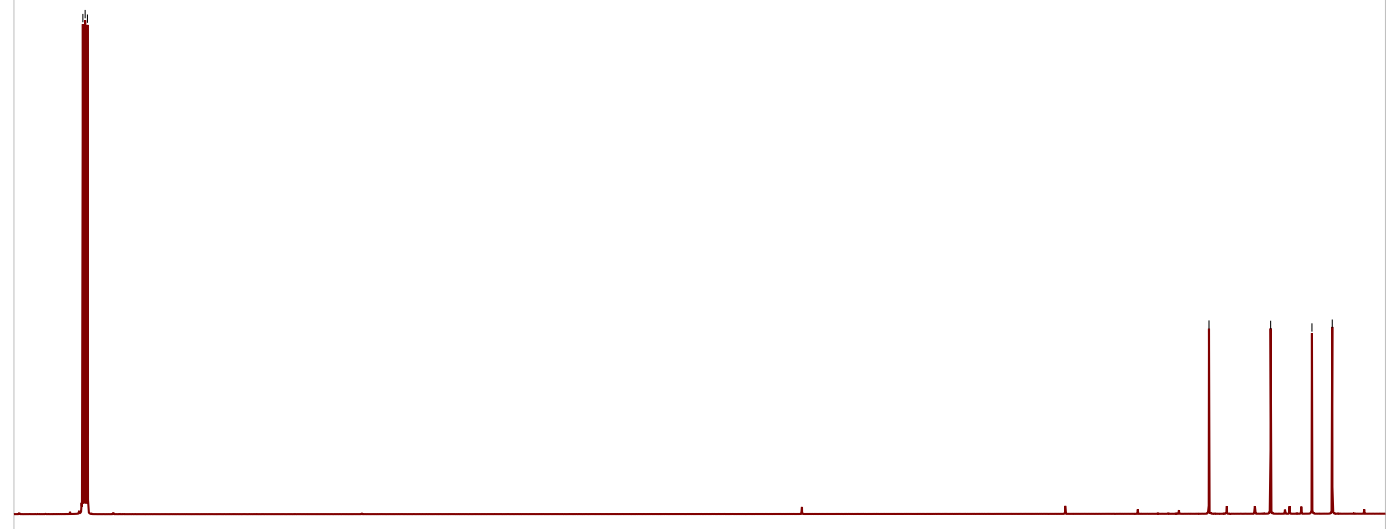

$\begin{array}{llllllllllllllllllllllllll}130 & 125 & 120 & 115 & 110 & 105 & 100 & 95 & 90 & 85 & \begin{array}{c}80 \\ \mathrm{f} 1(\mathrm{ppm})\end{array} & 70 & 65 & 60 & 55 & 50 & 45 & 40 & 35 & 30 & 25 & 21\end{array}$

Figure S21. ${ }^{13} \mathrm{C}$ NMR spectrum $\left(128 \mathrm{MHz}\right.$, benzene- $\left.d_{6}, \mathbf{2 3}^{\circ} \mathrm{C}\right)$ of cis-bicyclo[4.2.0]octane.

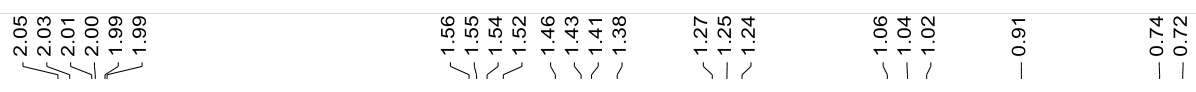

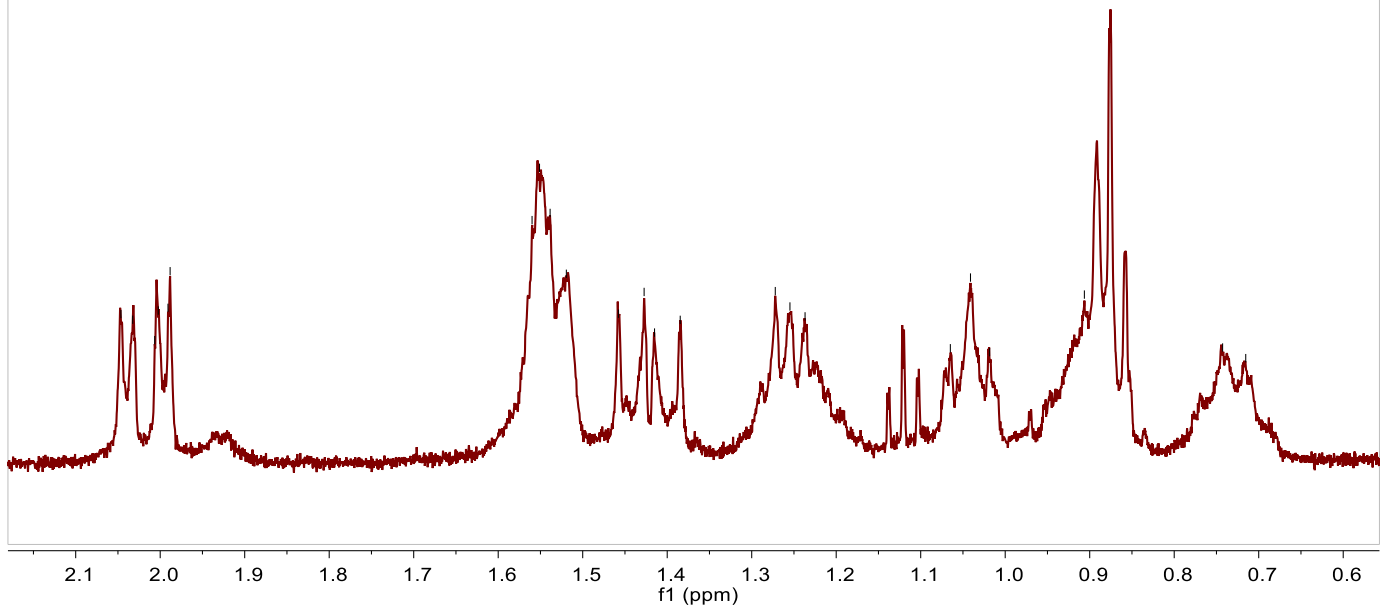

Figure S22. ${ }^{1} \mathrm{H}$ NMR spectrum $\left(400 \mathrm{MHz}\right.$, benzene- $\left.d_{6}, 2^{\circ}{ }^{\circ} \mathrm{C}\right)$ of trans-bicyclo[4.2.0]octane. Contains diethyl ether and pentane. 

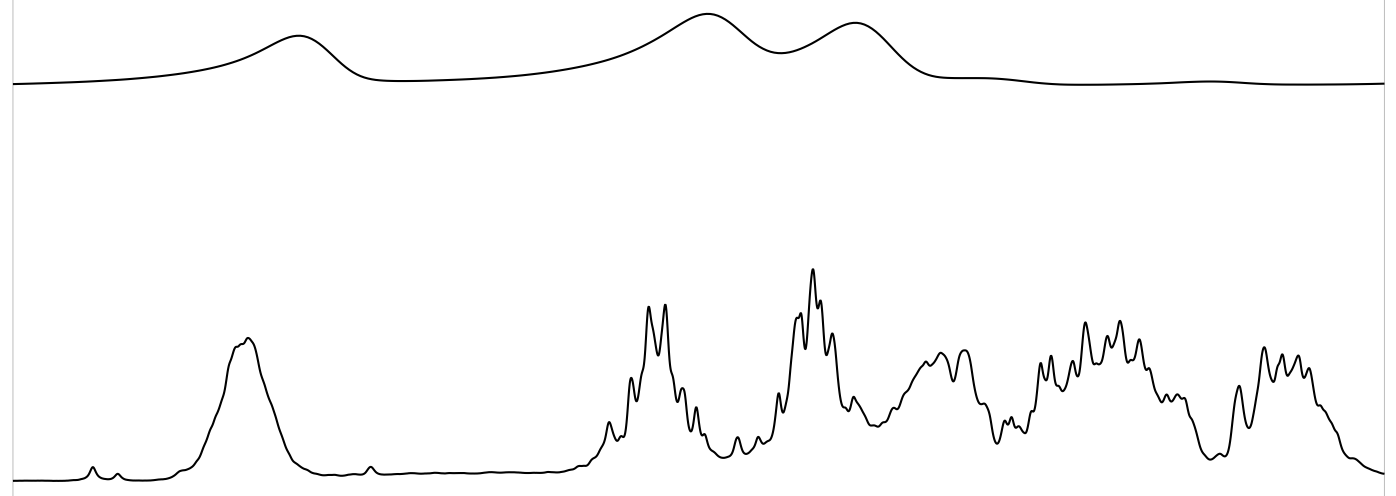

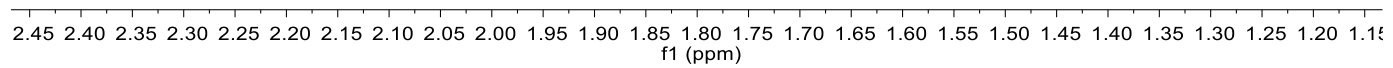

Figure S23. Top - ${ }^{2} \mathrm{H}$ NMR spectrum (77 MHz, benzene, $23{ }^{\circ} \mathrm{C}$ ) of cis-bicyclo[4.2.0]octane- $d_{n}$. Bottom - reference spectrum of the protio version.

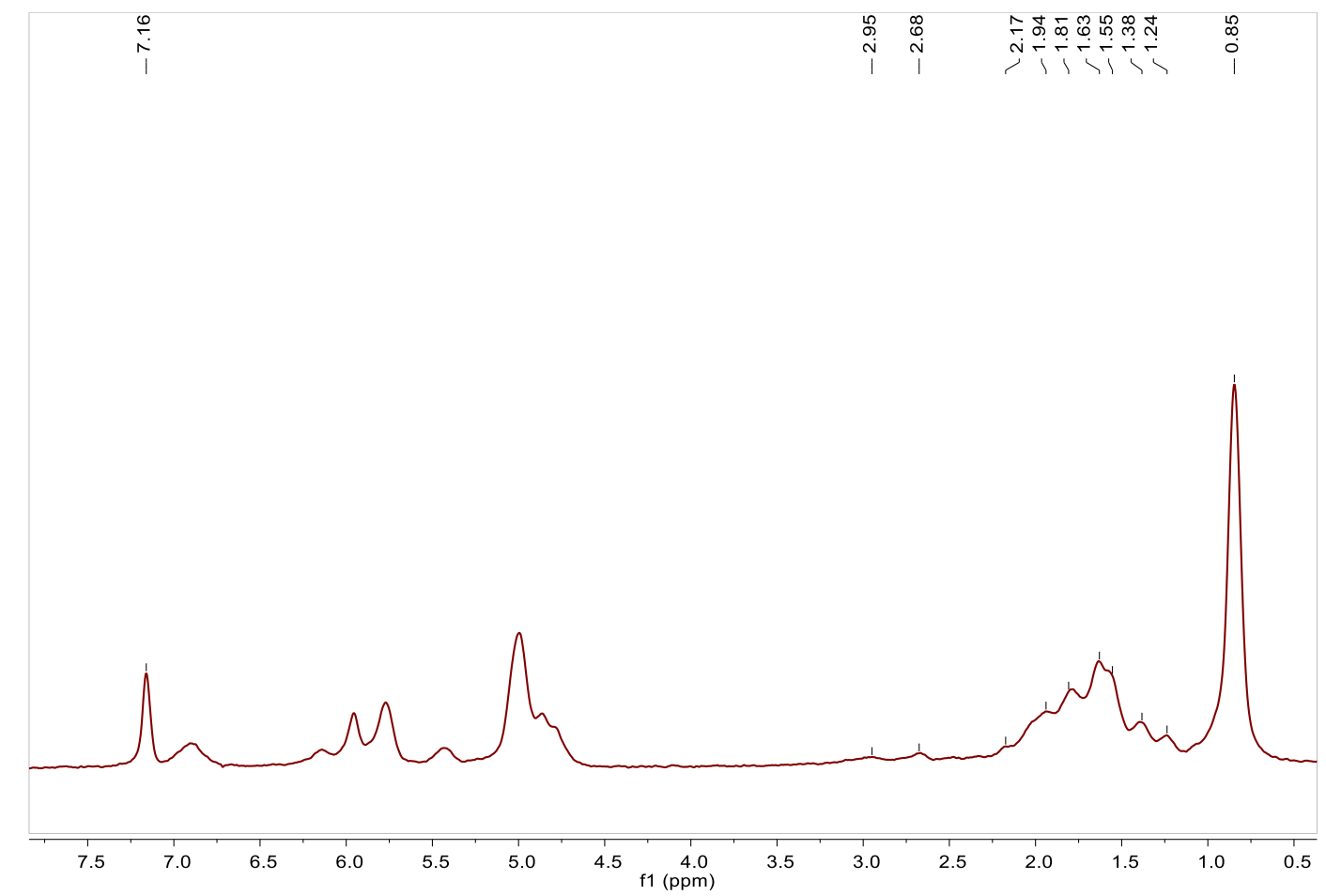

Figure S24. ${ }^{2} \mathrm{H}$ NMR spectrum $\left(77 \mathrm{MHz}\right.$, benzene, $\left.23^{\circ} \mathrm{C}\right)$ of tricPDI after reaction with deuterated 1,7-octadiene. Contains dideutero 1,7-octadiene and isomerized diene. 


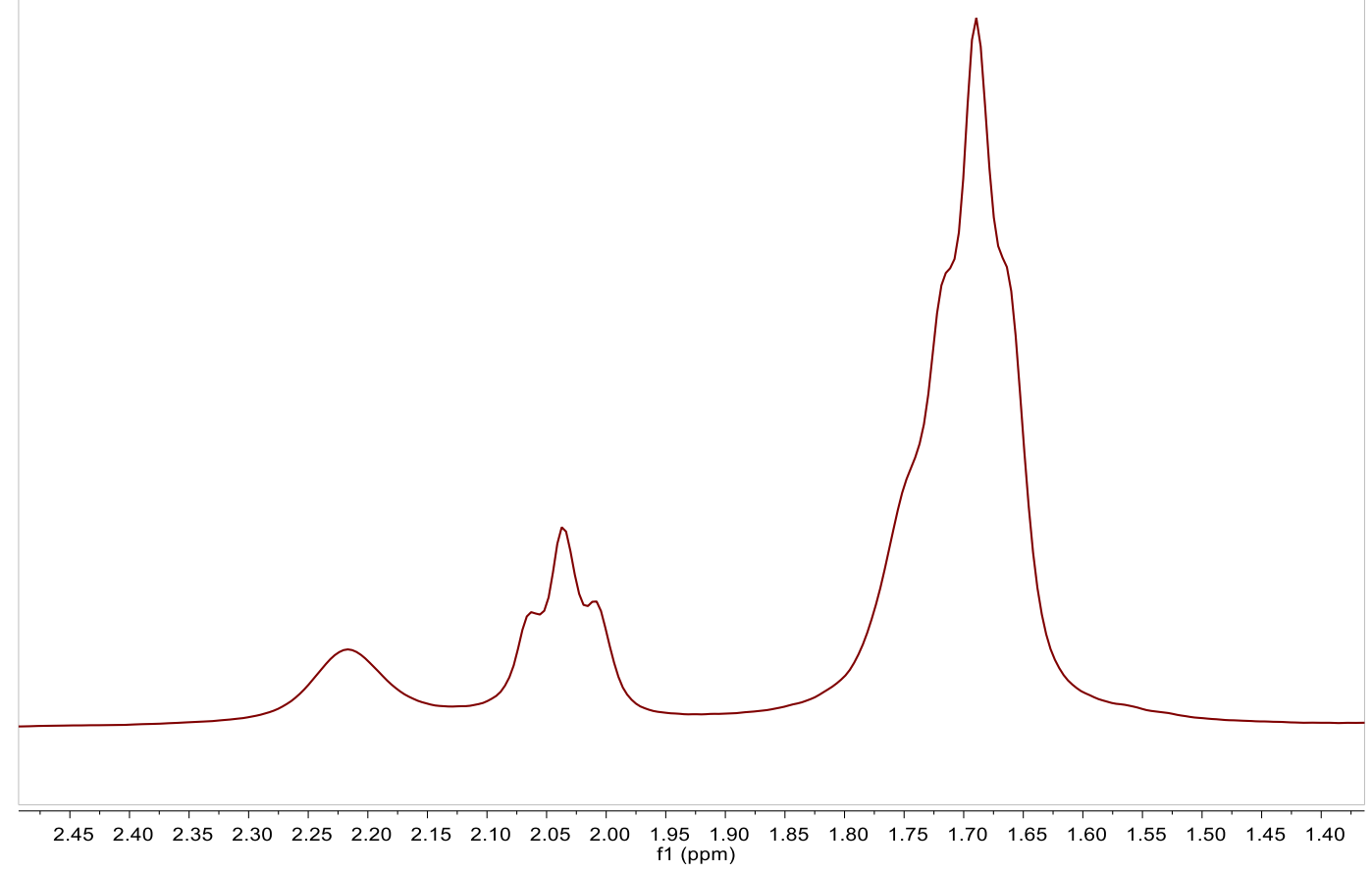

Figure S25. ${ }^{2} \mathrm{H}$ NMR spectrum (77 MHz, benzene, $23{ }^{\circ} \mathrm{C}$ ) of 3-(4-fluorophenyl)-3azabicyclo[3.2.0]heptane- $d_{n}$. \% incorporation and isotopomeric ratios calculated here.

\section{Additional Spectra}

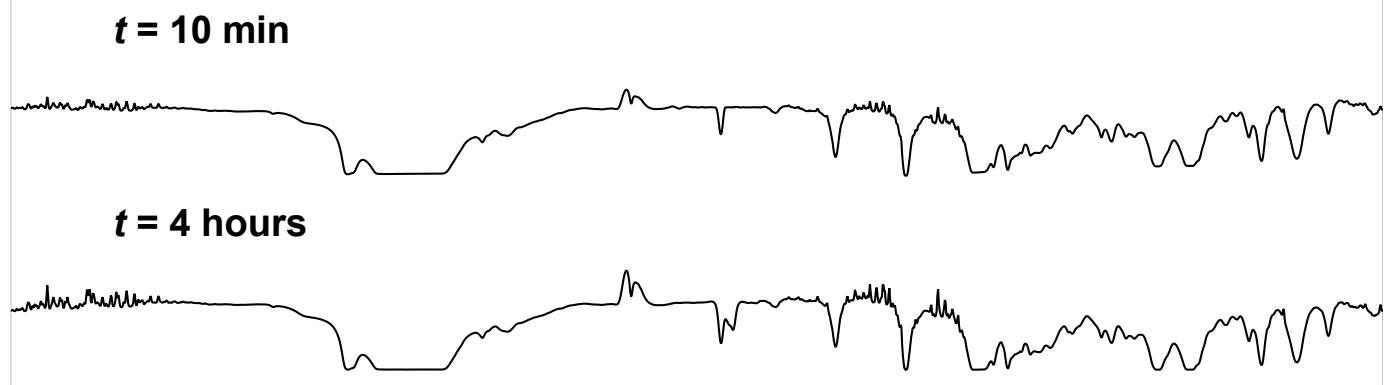

$t=18$ hours

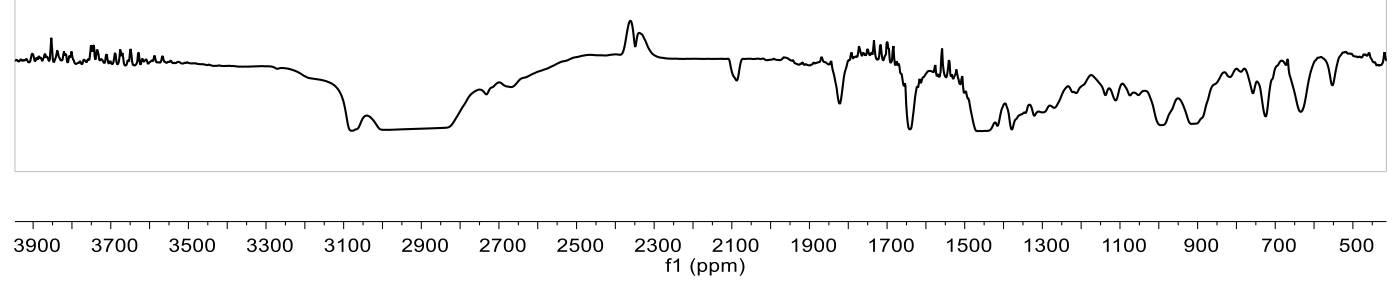

Figure S26. IR spectra from Figure 7 before subtraction of 1-octene reference spectrum. 


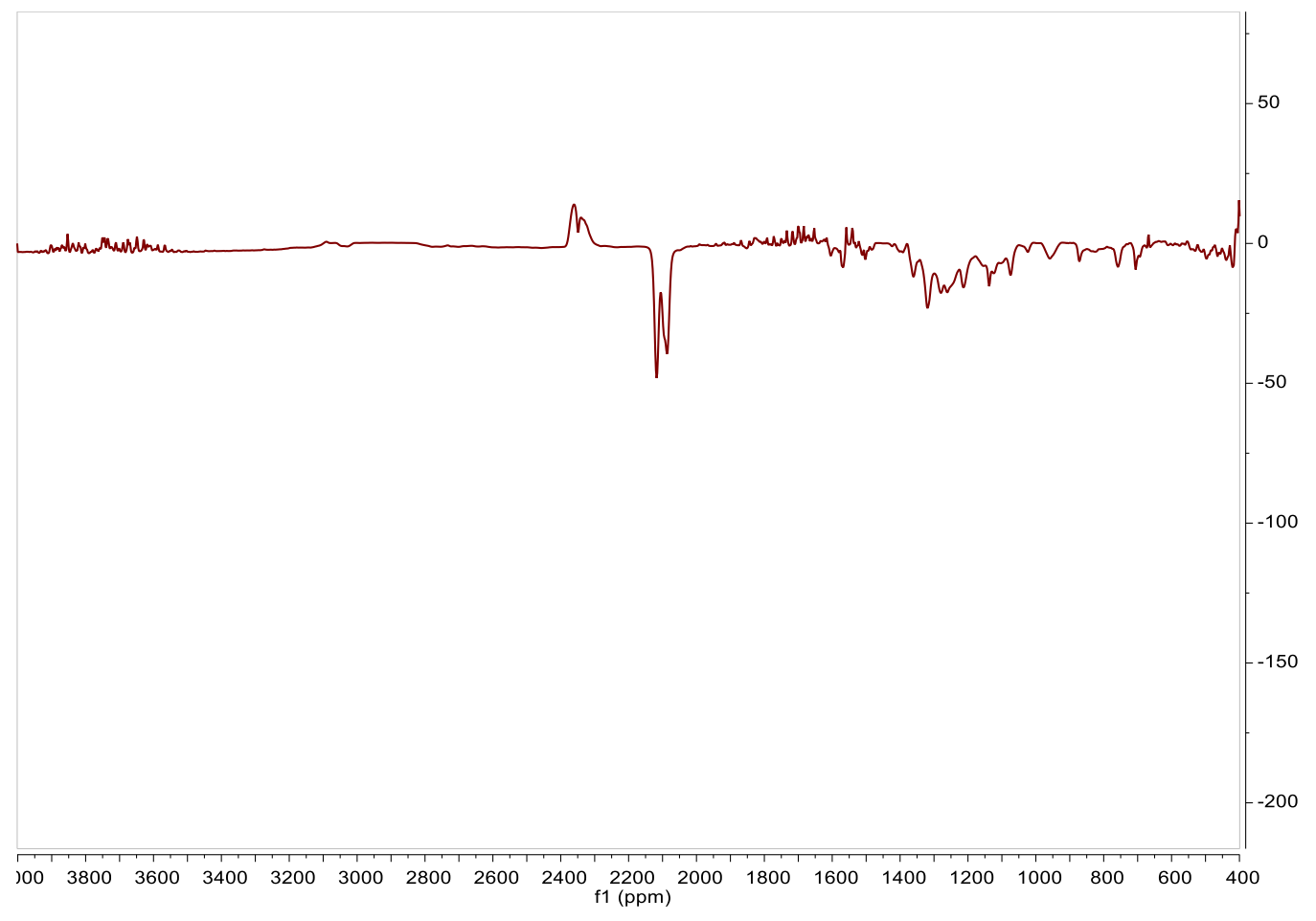

Figure S27. Example IR spectrum from Figure 7 after subtraction of 1-octene reference spectrum (4 hour time point).

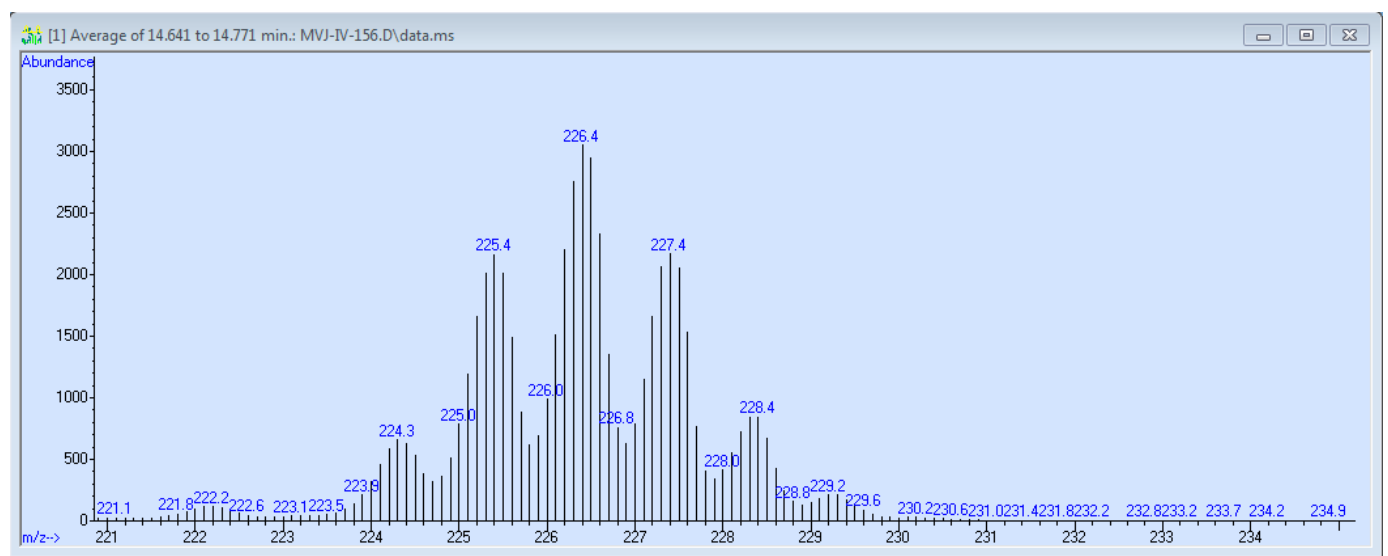

Figure S28. GC-MS of trans-1,2-dihexylcyclobutane from reaction in Figure 14. 


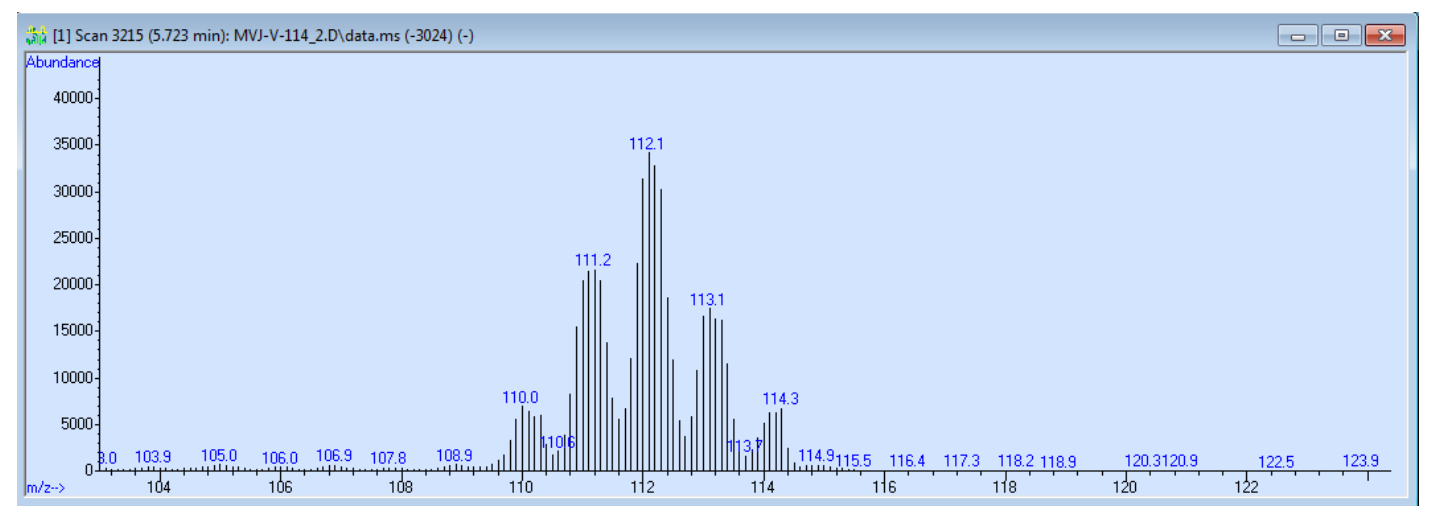

Figure S29. GC-MS of cis-bicyclo[4.2.0]octane from reaction in Figure 15.

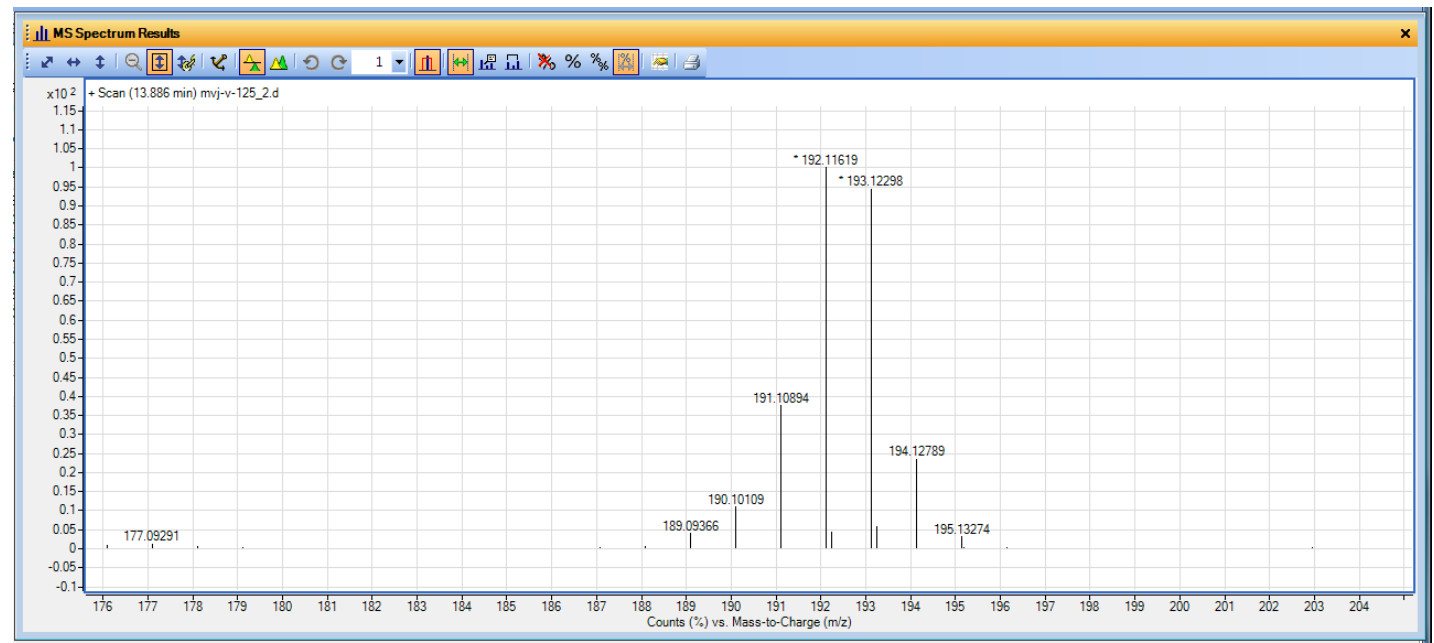

Figure S30. GC-MS of 3-(4-fluorophenyl)-3-azabicyclo[3.2.0]heptane from reaction in Figure 16. 


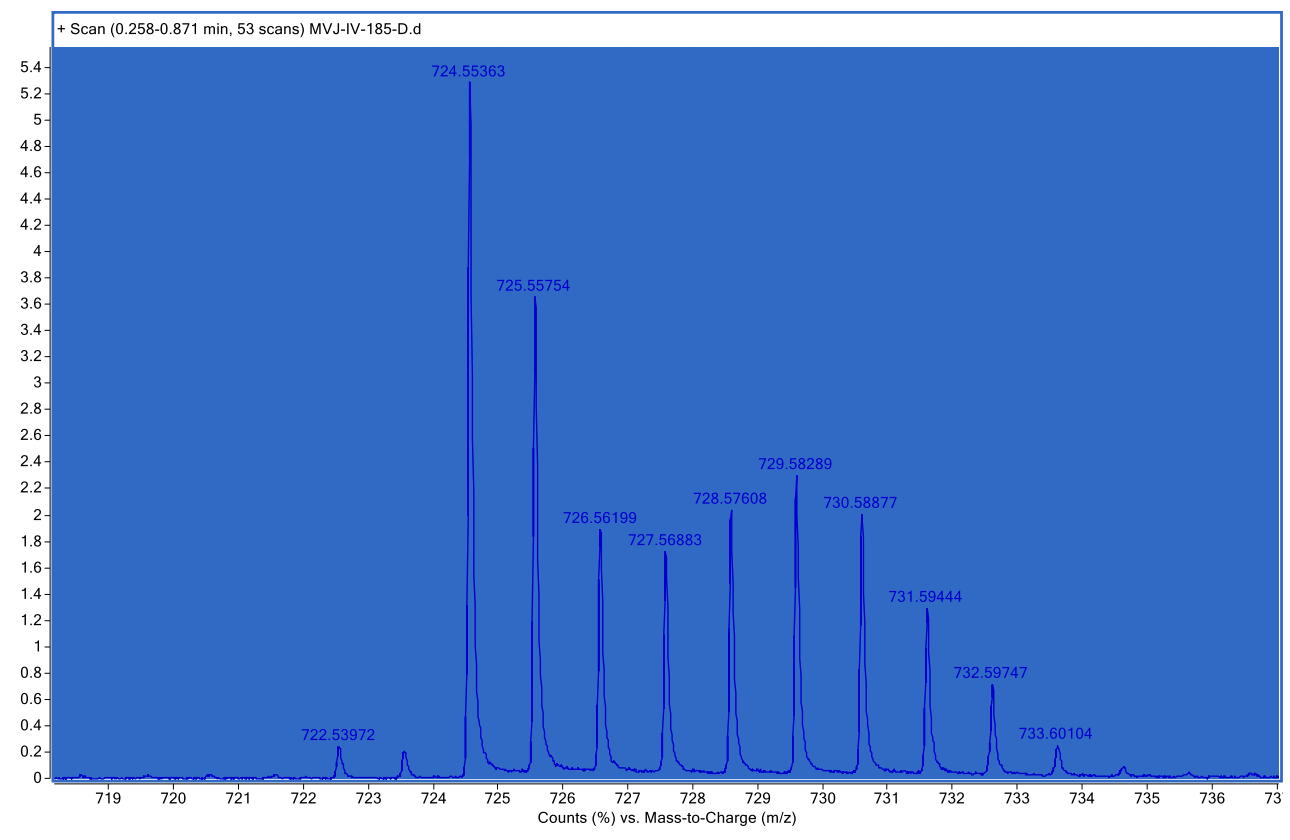

Figure S31. LC-MS of tricPDI from reaction in Figure 15. 


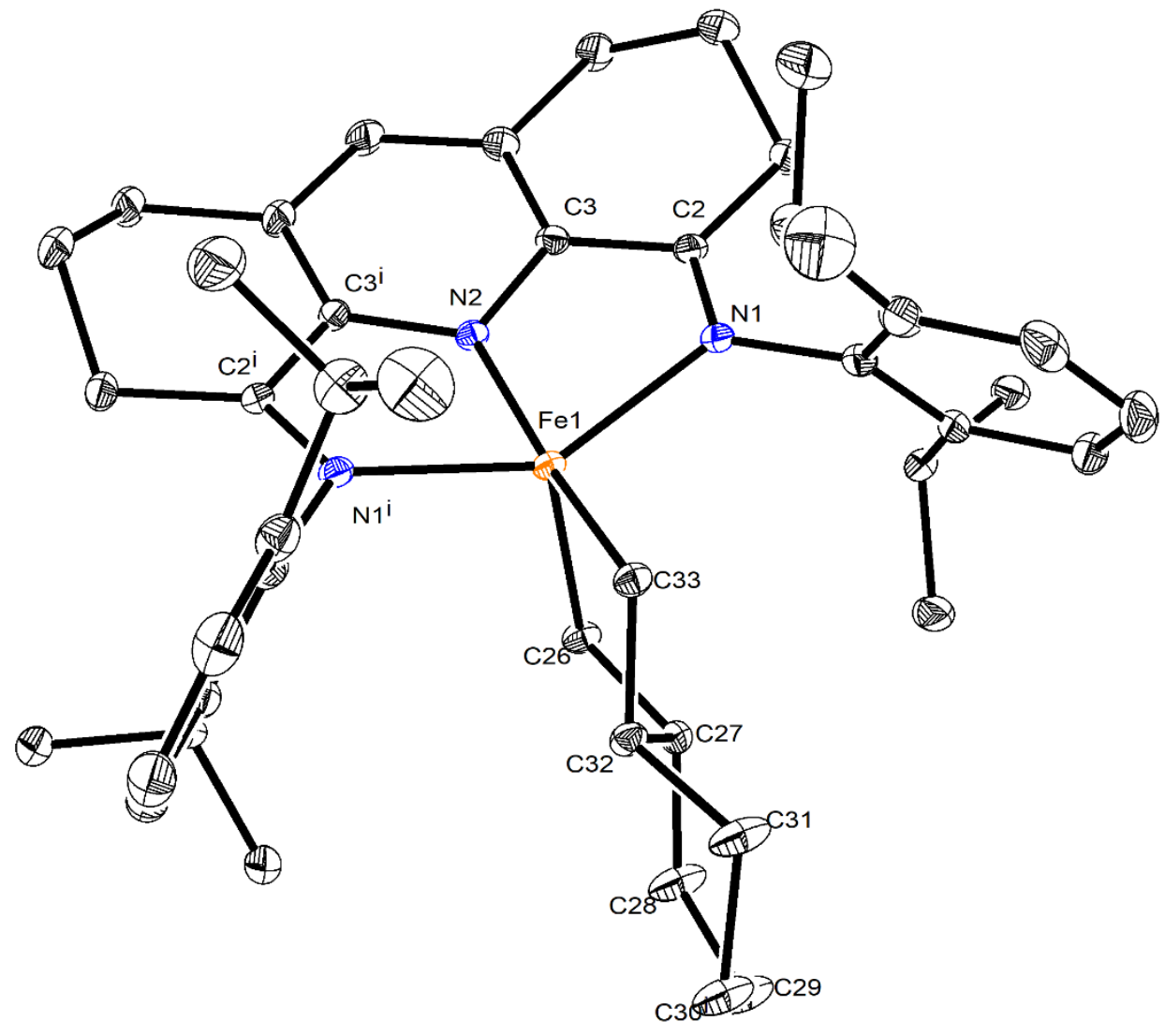

Figure S32. Representation of the solid-state structure of ( $\left.{ }^{(\mathrm{Pr}(\mathrm{TB})} \mathrm{PDI}\right) \mathrm{Fe}($ bimetallacyclo[4.3.0]nonane) at $30 \%$ thermal ellipsoids. Hydrogen atoms and a pentane molecule contained within the unit cell are omitted for clarity. 


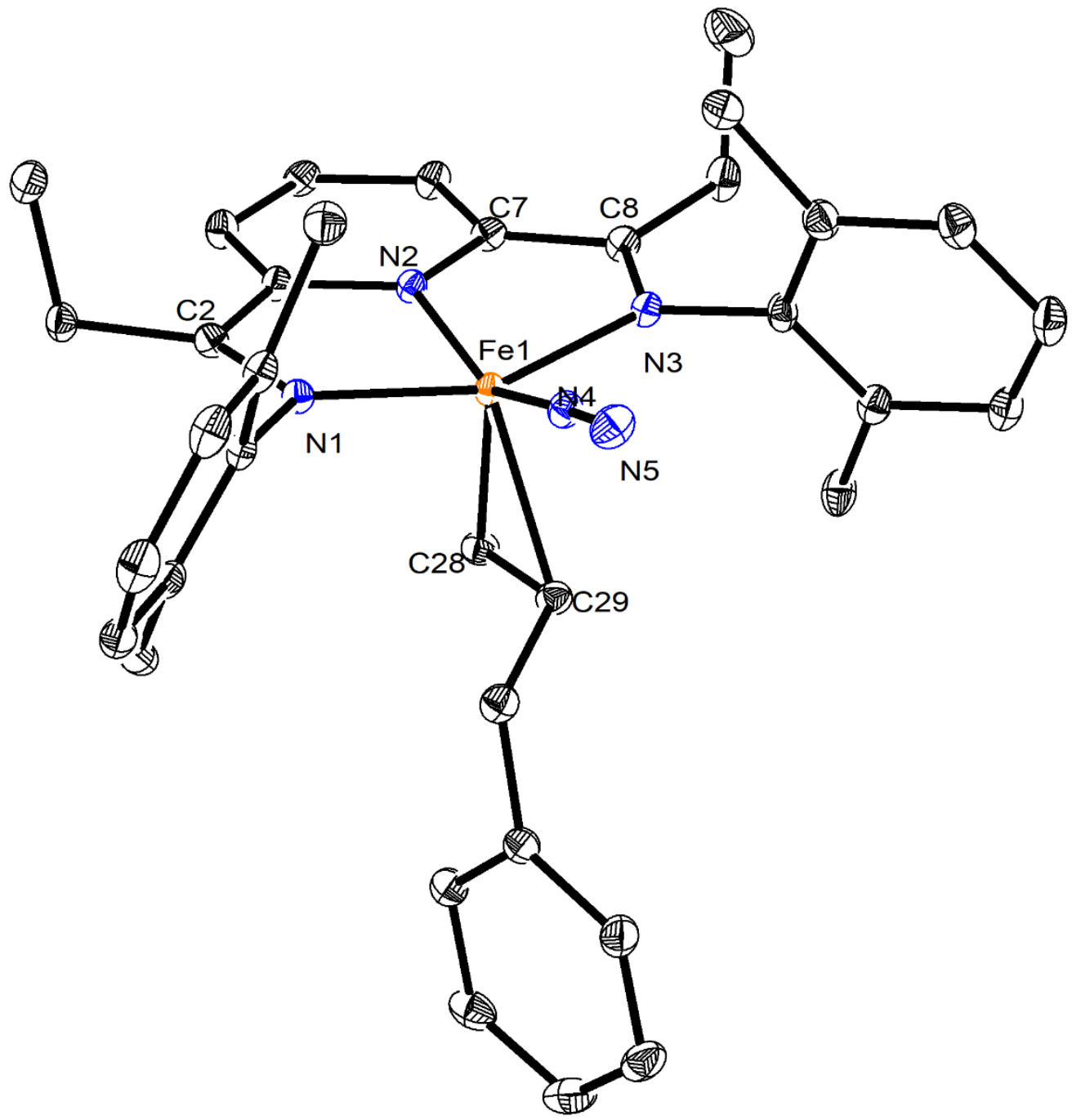

Figure S33. Representation of the solid-state structure of (Et-MePDI)Fe( $\eta^{2}$-allylbenzene $)\left(\mathrm{N}_{2}\right)$ at $30 \%$ thermal ellipsoids. Hydrogen atoms and a pentane molecule contained within the unit cell are omitted for clarity. 


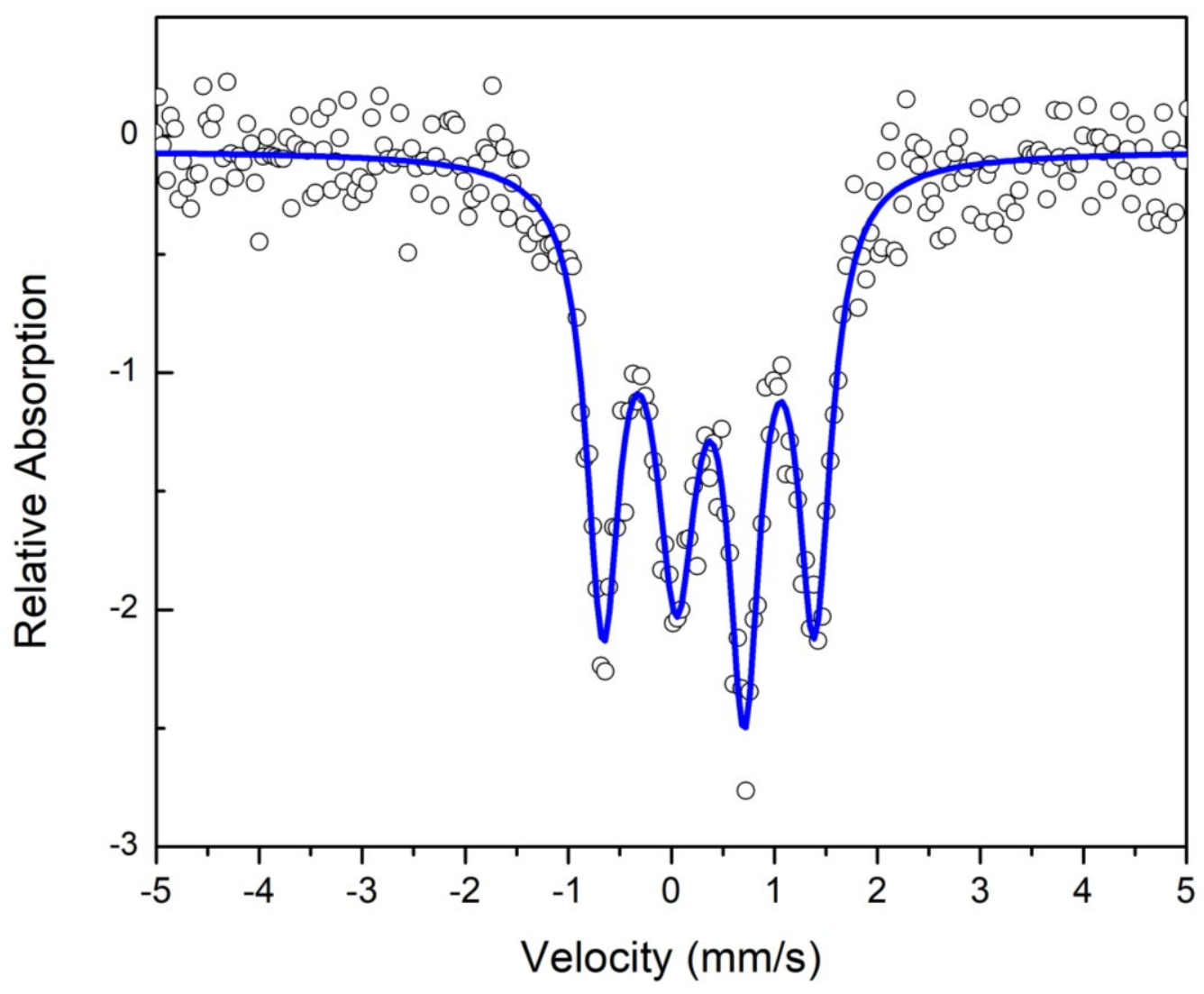

Figure S34. Solution-state zero-field ${ }^{57} \mathrm{Fe}$ Mössbauer spectrum of [2+2] catalytic reaction of 1octene after 10 minutes. Sample was not allowed to settle before sample was freeze-quenched to $196{ }^{\circ} \mathrm{C}$. Sample is $\sim 1: 1$ mixture of (tricPDI)Fe $\left(\mathrm{N}_{2}\right)\left(\eta^{2}-1\right.$-octene) and (tric PDI)Fe $\left(\mathrm{N}_{2}\right)$. Acquisition temperature: $80 \mathrm{~K}$. Species $1: \delta=0.38 \mathrm{~mm} / \mathrm{s} ;|\Delta \mathrm{E}|=0.66 \mathrm{~mm} / \mathrm{s}$. Species $2: \delta=0.37 \mathrm{~mm} / \mathrm{s} ;|\Delta \mathrm{E}|$ $=2.07 \mathrm{~mm} / \mathrm{s}$. 


\section{DFT Input Files and Coordinates}

- DFT Input File - 1-octene

! RKS B3LYP RIJCOSX def2-SVP def2/J Normalprint SlowConv TightSCF TightOpt NumFreq Grid5 FinalGrid6 GridX4 Pal8 UCO

\begin{tabular}{|c|c|c|c|}
\hline \multicolumn{4}{|c|}{$\begin{array}{l}\text { \%SCF Maxlter } 5000 \\
\text { Convergence VeryTight } \\
\text { end }\end{array}$} \\
\hline \multicolumn{4}{|c|}{$\begin{array}{l}\text { \%freq increment } 0.01 \\
\text { restart true } \\
\text { centraldiff true } \\
\text { end }\end{array}$} \\
\hline \multicolumn{4}{|c|}{ * xyz 01} \\
\hline C & -6.65591 & 0.15998 & -0.00012 \\
\hline C & -5.35835 & -0.16540 & 0.01129 \\
\hline C & -4.24444 & 0.84612 & 0.00732 \\
\hline C & -2.88039 & 0.15291 & 0.01290 \\
\hline $\mathrm{H}$ & -4.33097 & 1.48823 & 0.89197 \\
\hline $\mathrm{H}$ & -4.32934 & 1.48017 & -0.88321 \\
\hline C & -1.71379 & 1.14287 & -0.00739 \\
\hline $\mathrm{H}$ & -2.80361 & -0.51010 & -0.85864 \\
\hline $\mathrm{H}$ & -2.79852 & -0.48221 & 0.90448 \\
\hline C & -0.38527 & 0.38321 & 0.01874 \\
\hline $\mathrm{H}$ & -1.77703 & 1.81355 & 0.85782 \\
\hline $\mathrm{H}$ & -1.76958 & 1.76559 & -0.90835 \\
\hline C & 0.82199 & 1.31726 & -0.00659 \\
\hline $\mathrm{H}$ & -0.33808 & -0.29490 & -0.84233 \\
\hline $\mathrm{H}$ & -0.34193 & -0.24050 & 0.92029 \\
\hline C & 2.12023 & 0.52326 & 0.00426 \\
\hline $\mathrm{H}$ & 0.79832 & 1.98404 & 0.86290 \\
\hline $\mathrm{H}$ & 0.79116 & 1.94704 & -0.90300 \\
\hline $\mathrm{H}$ & 2.18875 & -0.10358 & 0.89947 \\
\hline $\mathrm{H}$ & 2.98156 & 1.19783 & -0.00560 \\
\hline $\mathrm{H}$ & 2.18779 & -0.12764 & -0.87373 \\
\hline $\mathrm{H}$ & -7.41951 & -0.61266 & 0.00257 \\
\hline $\mathrm{H}$ & -6.98578 & 1.19397 & -0.01213 \\
\hline $\mathrm{H}$ & -5.08938 & -1.22051 & 0.02335 \\
\hline
\end{tabular}

end

- Calculated Thermochemical Properties - 1-octene (Calculated at 298.15 K)

Total enthalpy $\quad \ldots \quad-313.784760902$ Eh

Total entropy correction $\quad \ldots \quad-0.04572933$ Eh $\quad-28.70 \mathrm{kcal} / \mathrm{mol}$

Final Gibbs free enthalpy $\quad \ldots \quad-313.82954202$ Eh 


\section{- Optimized Coordinates -1-octene}

$\begin{array}{llcc}\text { C } & -6.48356768438693 & 0.00811479200210 & -0.54885881866491 \\ \text { C } & -5.47955977569126 & -0.00785602442218 & 0.33313898324013 \\ \text { C } & -4.28621878097766 & 0.90726118203845 & 0.31943117350811 \\ \text { C } & -2.94817660636706 & 0.16398344116506 & 0.16386409791926 \\ \text { H } & -4.25990811662194 & 1.48231835770526 & 1.26557652152607 \\ \text { H } & -4.39555450599289 & 1.64959897340842 & -0.49080643412644 \\ \text { C } & -1.72843872131791 & 1.09083112797884 & 0.17338732355957 \\ \text { H } & -2.96637304623645 & -0.41393432014571 & -0.77806658778538 \\ \text { H } & -2.84850862911500 & -0.58352799832380 & 0.97320374024161 \\ \text { C } & -0.39474643880072 & 0.35990794004028 & -0.00808069408043 \\ \text { H } & -1.70856863867376 & 1.66008682075354 & 1.12205672547390 \\ \text { H } & -1.84002471362750 & 1.84757915874572 & -0.62597035223403 \\ \text { C } & 0.82587286062305 & 1.28671284721440 & -0.01394717153098 \\ \text { H } & -0.42009083925655 & -0.21389903655316 & -0.95391890453447 \\ \text { H } & -0.27750812644326 & -0.39335738057161 & 0.79406632314521 \\ \text { C } & 2.15189579786622 & 0.55136903365072 & -0.21557227047540 \\ \text { H } & 0.85719217215456 & 1.85168139661060 & 0.93614192367335 \\ \text { H } & 0.70045375146923 & 2.04589459775667 & -0.80812215842250 \\ \text { H } & 2.32572256488343 & -0.18950753163965 & 0.58339093840943 \\ \text { H } & 3.00655889551968 & 1.24747095644306 & -0.21602080110717 \\ \text { H } & 2.16616857546755 & 0.00718706028983 & -1.17533280511610 \\ \text { H } & -7.31914204218702 & -0.69502374430554 & -0.48032767191547 \\ \text { H } & -6.51082927970026 & 0.73006582353276 & -1.37305297104239 \\ \text { H } & -5.49872867258754 & -0.75442747337408 & 1.14008989033905\end{array}$

\section{- DFT Input File - trans-1,2-dihexylcyclobutane}

! RKS B3LYP RIJCOSX def2-SVP def2/J Normalprint SlowConv TightSCF TightOpt NumFreq Grid5 FinalGrid6 GridX4 Pal8 UCO

\begin{tabular}{|c|c|c|c|}
\hline \multicolumn{4}{|c|}{$\begin{array}{l}\text { \%SCF Maxlter } 5000 \\
\text { Convergence VeryTight } \\
\text { end }\end{array}$} \\
\hline \multicolumn{4}{|c|}{$\begin{array}{l}\text { \%freq increment } 0.01 \\
\text { restart true } \\
\text { centraldiff true } \\
\text { end }\end{array}$} \\
\hline \multicolumn{4}{|c|}{ * xyz 01} \\
\hline C & -6.74082 & -0.14384 & 0.09856 \\
\hline C & -5.31748 & -0.41358 & -0.44157 \\
\hline C & -4.27711 & 0.58735 & 0.08813 \\
\hline C & -2.85655 & 0.07031 & -0.11290 \\
\hline $\mathrm{H}$ & -4.43581 & 0.78006 & 1.15628 \\
\hline $\mathrm{H}$ & -4.40411 & 1.54263 & -0.43436 \\
\hline 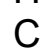 & -1.76071 & 1.10206 & 0.14756 \\
\hline $\mathrm{H}$ & -2.73798 & -0.27413 & -1.14431 \\
\hline $\mathrm{H}$ & -2.69867 & -0.78771 & \\
\hline & -0.38992 & 0.41805 & 0.06810 \\
\hline
\end{tabular}




\begin{tabular}{lrrr} 
H & -1.89084 & 1.56126 & 1.13428 \\
$\mathrm{H}$ & -1.82830 & 1.89991 & -0.60079 \\
$\mathrm{C}$ & 0.76377 & 1.41655 & 0.07919 \\
$\mathrm{H}$ & -0.33023 & -0.18591 & -0.84675 \\
$\mathrm{H}$ & -0.28555 & -0.27539 & 0.91202 \\
$\mathrm{C}$ & 2.10584 & 0.69989 & 0.03499 \\
$\mathrm{H}$ & 0.71412 & 2.04051 & 0.97849 \\
$\mathrm{H}$ & 0.68453 & 2.08257 & -0.78751 \\
$\mathrm{H}$ & 2.23780 & 0.06104 & 0.91424 \\
$\mathrm{H}$ & 2.92498 & 1.42508 & 0.01536 \\
$\mathrm{H}$ & 2.18723 & 0.07160 & -0.85842 \\
$\mathrm{C}$ & -6.92969 & -1.64719 & 0.33166 \\
$\mathrm{H}$ & -7.43065 & 0.30034 & -0.62549 \\
$\mathrm{H}$ & -6.78250 & 0.44344 & 1.02279 \\
$\mathrm{C}$ & -5.40645 & -1.85479 & 0.17070 \\
$\mathrm{H}$ & -7.54727 & -2.12783 & -0.43534 \\
$\mathrm{H}$ & -7.32930 & -1.91301 & 1.31509 \\
$\mathrm{H}$ & -5.30412 & -0.43462 & -1.54137 \\
$\mathrm{C}$ & -5.04929 & -3.06238 & -0.71368 \\
$\mathrm{H}$ & -4.90157 & -1.92132 & 1.14548 \\
$\mathrm{C}$ & -3.60147 & -2.96154 & -1.17207 \\
$\mathrm{H}$ & -5.70319 & -3.10302 & -1.59341 \\
$\mathrm{H}$ & -5.20490 & -3.98833 & -0.14854 \\
$\mathrm{C}$ & -3.07247 & -4.13658 & -1.98913 \\
$\mathrm{H}$ & -2.95609 & -2.82774 & -0.29661 \\
$\mathrm{H}$ & -3.51003 & -2.07011 & -1.79789 \\
$\mathrm{C}$ & -1.64253 & -3.79874 & -2.43217 \\
$\mathrm{H}$ & -3.70730 & -4.30514 & -2.86687 \\
$\mathrm{H}$ & -3.08169 & -5.05167 & -1.38747 \\
$\mathrm{C}$ & -0.97192 & -4.91613 & -3.22134 \\
$\mathrm{H}$ & -1.03461 & -3.57071 & -1.54678 \\
$\mathrm{H}$ & -1.66093 & -2.88720 & -3.04360 \\
$\mathrm{C}$ & 0.43080 & -4.49929 & -3.64383 \\
$\mathrm{H}$ & -1.56295 & -5.15729 & -4.11188 \\
$\mathrm{H}$ & -0.91234 & -5.82182 & -2.60813 \\
$\mathrm{H}$ & 0.39540 & -3.63680 & -4.31795 \\
$\mathrm{H}$ & 0.93414 & -5.31841 & -4.16534 \\
& 1.03804 & -4.22454 & -2.77452 \\
& & & \\
\hline
\end{tabular}

end

- Calculated Thermochemical Properties - trans-1,2-dihexylcyclobutane (Calculated at 298.15 K)

Total enthalpy $\quad$... $\quad-627.59246969$ Eh

Total entropy correction $\quad \ldots \quad-0.06768197 \mathrm{Eh} \quad-42.47 \mathrm{kcal} / \mathrm{mol}$

Final Gibbs free enthalpy $\quad \ldots \quad-627.66015165 \mathrm{Eh}$ 


\section{- Optimized Coordinates - trans-1,2-dihexylcyclobutane}

\begin{tabular}{|c|c|c|c|}
\hline C & -6.68958164555534 & -0.14964208069042 & 0.17692671432630 \\
\hline & -5.23716140670853 & -0.34155945855639 & -0.34563111837859 \\
\hline & 17595283879900 & 4766091083702 & \\
\hline & -2.73431032617337 & 33512482286728 & 306826 \\
\hline & -4.22342481608194 & 0.41441947935018 & 1.40081023548439 \\
\hline & -4.45935763653887 & 1.60246229812047 & 0.1220787838052 \\
\hline & -1.73252186984225 & 1.31129175220414 & 0.4589116122765 \\
\hline & -2.69087280079615 & 0.42737354315313 & -1.2694307134965 \\
\hline & -2.42009315565719 & -0.69860509265061 & 0.060318760737 \\
\hline & 8090761507 & 110383992006 & \\
\hline & 7635412265 & 1.23742128274040 & \\
\hline & -2.02995250555955 & 2.34860612327196 & 0.21398955927907 \\
\hline & 0.72068671135149 & 2.06022253026099 & 0.63068784921503 \\
\hline & -0.22601016223219 & 1.14483232704922 & -1.08806317586016 \\
\hline & 0.01 & 0245 & 0.26887188768822 \\
\hline & 2.16 & 1.8 & \\
\hline & 0.65 & & \\
\hline & | 64995667 & 0591 & 4137 \\
\hline & 2.50128147756251 & 8614 & 0.47428 \\
\hline & 2.85954778224501 & 2.5333294 & 0.65133901 \\
\hline & 484097 & 1.89 & -0.90368683 \\
\hline & -6.8 & -1.6 & \\
\hline & -7.3 & & \\
\hline & 8226131 & 569 & \\
\hline & -5.33047000538193 & -1.86414503521404 & 0.026887 \\
\hline & -7.45717138877450 & -2.10293112963214 & -0.550837 \\
\hline & $\$ 19540568674$ & -2.083 & 02788656 \\
\hline & $-5.1 \varsigma$ & -0.2 & -1.444 \\
\hline & -4.9 & -2.9 & -0 . \\
\hline & -4.7 & -2.0 & \\
\hline & -3.45149662701524 & 13046 & -1.260933 \\
\hline $\mathrm{H}$ & -5.45983332900508 & -2.70554620051410 & 3291896 \\
\hline & -5.37435289539103 & 597012663352 & $-0.658891227490 \varsigma$ \\
\hline & $-3.1<$ & -4.2 & -2.2484 \\
\hline & -2.9 & -3.3 & -0.3 \\
\hline & -3.0 & -2.1 & -1. \\
\hline C & 7608801928 & -4.4 & $-2.517 c$ \\
\hline $\mathrm{H}$ & -3.66256655643515 & -4.05262356578763 & -3.20502902 \\
\hline & -3.57827824237388 & -5.19836769248493 & -1.8691028779148 \\
\hline & -1.34411139390670 & -5.57959228841507 & -3.5136597055788 \\
\hline & & -4.6 & \\
\hline & -1.21386755142088 & -3.5100928 & -2.8897897101998 \\
\hline & 0.15018164308019 & -5.77868555656090 & -3.77394616296180 \\
\hline $\mathrm{H}$ & -1.85999904363298 & -5.36730216710912 & -4.4682364360458 \\
\hline & -1.78318524022487 & -6.52416786665301 & -3.1424050872856 \\
\hline & & -4.86084821764097 & -4.17444172832515 \\
\hline & & -6.58693433378979 & -4.50102 \\
\hline & 0.68824487956178 & -6.03839951524875 & -2.846308859599 \\
\hline
\end{tabular}


- DFT Input File - (dicPDI)Fe $\left(\mathrm{N}_{2}\right)\left(\eta^{2}-1\right.$-octene $)$

! RKS B3LYP RIJCOSX def2-SVP def2/J Normalprint SlowConv TightSCF Opt Pal8 UCO

\%basis NewGTO 26 "def2-TZVP(-f)" end

NewGTO 7 "def2-TZVP(-f)" end

NewAuxGTO 26 "def2/J" end

NewAuxGTO 7 "def2/J" end end

$\%$ SCF Maxlter 8000

TolE $1 \mathrm{e}-7$

TolErr 1e-6

end

${ }^{*} \mathrm{xyz} 01$

$\begin{array}{lrrr}\mathrm{C} & 3.60823 & 1.74027 & -0.05474 \\ \mathrm{C} & 2.28592 & 1.49632 & -0.01301 \\ \mathrm{~N} & 1.94689 & 0.23895 & -0.01207 \\ \mathrm{C} & 2.72793 & -0.78993 & -0.07491 \\ \mathrm{C} & 4.04923 & -0.56202 & -0.11157 \\ \mathrm{C} & 4.47750 & 0.71306 & -0.09422 \\ \mathrm{C} & 1.26560 & 2.39638 & -0.00948 \\ \mathrm{C} & 2.03597 & -1.94298 & -0.11534 \\ \mathrm{C} & 1.57808 & 3.87012 & -0.17989 \\ \mathrm{~N} & 0.00000 & 2.00216 & 0.00000 \\ \mathrm{C} & 2.76148 & -3.26058 & -0.28201 \\ \mathrm{~N} & 0.71890 & -1.86268 & -0.08919 \\ \mathrm{C} & -0.99088 & 2.82034 & 0.30516 \\ \mathrm{C} & 0.03084 & -2.97103 & 0.12342 \\ \mathrm{C} & -2.01379 & 2.99783 & -0.57976 \\ \mathrm{C} & -3.08679 & 3.73888 & -0.25080 \\ \mathrm{C} & -3.19106 & 4.33068 & 0.93904 \\ \mathrm{C} & -2.15006 & 4.24034 & 1.76765 \\ \mathrm{C} & -1.04445 & 3.52626 & 1.47752 \\ \mathrm{C} & -0.77300 & -3.48585 & -0.84957 \\ \mathrm{C} & -1.52923 & -4.57113 & -0.60515 \\ \mathrm{C} & -1.52517 & -5.17667 & 0.58313 \\ \mathrm{C} & -0.68230 & -4.72215 & 1.51198 \\ \mathrm{C} & 0.11410 & -3.65606 & 1.30459 \\ \mathrm{C} & -1.92368 & 2.63220 & -2.05471 \\ \mathrm{C} & 0.03554 & 3.60668 & 2.56081 \\ \mathrm{C} & 1.04625 & -3.32843 & 2.47148 \\ \mathrm{C} & -0.72296 & -3.04721 & -2.30422 \\ \mathrm{C} & -3.17695 & 2.19534 & -2.83424 \\ \mathrm{C} & -2.65340 & 2.14611 & -4.27907 \\ \mathrm{C} & -1.65139 & 3.31339 & -4.37442 \\ \mathrm{C} & -1.39346 & 3.78102 & -2.92882 \\ \mathrm{C} & 0.52198 & 5.03528 & 2.89470 \\ \mathrm{C} & 1.39856 & 4.83389 & 4.14153 \\ \mathrm{C} & 0.78407 & 3.62855 & 4.87650 \\ \mathrm{C} & -0.35244 & 3.11900 & 3.97182 \\ & & & \end{array}$




\begin{tabular}{|c|c|c|c|}
\hline C & 0.18930 & -3.92651 & -3.17569 \\
\hline C & -0.14205 & -3.50912 & -4.62170 \\
\hline C & -1.47277 & -2.73464 & -4.55407 \\
\hline C & -2.01396 & -3.00610 & -3.14123 \\
\hline C & 0.37701 & -2.94073 & 3.80423 \\
\hline C & 1.51534 & -3.00974 & 4.83839 \\
\hline C & 2.58394 & -3.93809 & 4.23252 \\
\hline C & 1.95805 & -4.48366 & 2.93881 \\
\hline $\mathrm{Fe}$ & 0.00000 & 0.00000 & 0.00000 \\
\hline C & -1.07612 & -0.54147 & 1.69675 NewGTO "def2-TZVP(-f)" end \\
\hline C & -2.16379 & 0.32171 & 2.31618 \\
\hline $\mathrm{N}$ & -1.90819 & -0.34673 & -0.68985 \\
\hline C & 0.48586 & 0.09495 & 2.01481 NewGTO "def2-TZVP(-f)" end \\
\hline C & -3.58392 & -0.27011 & 2.28062 \\
\hline C & -4.60798 & 0.69210 & 2.90577 \\
\hline C & -6.03467 & 0.12224 & 2.86169 \\
\hline C & -7.05863 & 1.09327 & 3.47050 \\
\hline C & -8.48329 & 0.52547 & 3.41975 \\
\hline $\mathrm{H}$ & 4.00342 & 2.76816 & -0.06372 \\
\hline $\mathrm{H}$ & 4.77605 & -1.38729 & -0.16567 \\
\hline $\mathrm{H}$ & 5.56066 & 0.91975 & -0.13107 \\
\hline $\mathrm{H}$ & 0.69593 & 4.54251 & -0.22786 \\
\hline $\mathrm{H}$ & 2.10102 & 4.01673 & -1.15273 \\
\hline $\mathrm{H}$ & 2.25638 & 4.23786 & 0.62099 \\
\hline $\mathrm{H}$ & 2.11856 & -4.16405 & -0.33081 \\
\hline $\mathrm{H}$ & 3.50088 & -3.40869 & 0.53581 \\
\hline $\mathrm{H}$ & 3.32447 & -3.24460 & -1.24311 \\
\hline $\mathrm{H}$ & -3.89979 & 3.93537 & -0.96435 \\
\hline $\mathrm{H}$ & -4.07140 & 4.94321 & 1.19555 \\
\hline $\mathrm{H}$ & -2.24467 & 4.79769 & 2.71230 \\
\hline $\mathrm{H}$ & -2.15016 & -5.03173 & -1.38740 \\
\hline $\mathrm{H}$ & -2.14700 & -6.06831 & 0.76868 \\
\hline $\mathrm{H}$ & -0.66292 & -5.27648 & 2.46305 \\
\hline $\mathrm{H}$ & -1.19195 & 1.81772 & -2.18350 \\
\hline $\mathrm{H}$ & 0.95469 & 3.05886 & 2.26358 \\
\hline $\mathrm{H}$ & 1.75384 & -2.51852 & 2.19754 \\
\hline $\mathrm{H}$ & -0.28657 & -2.03605 & -2.36314 \\
\hline $\mathrm{H}$ & -3.99001 & 2.95502 & -2.79981 \\
\hline $\mathrm{H}$ & -3.59936 & 1.22405 & -2.49574 \\
\hline $\mathrm{H}$ & -2.12471 & 1.17590 & -4.44308 \\
\hline $\mathrm{H}$ & -3.47178 & 2.21845 & -5.03118 \\
\hline $\mathrm{H}$ & -2.06079 & 4.14963 & -4.98791 \\
\hline $\mathrm{H}$ & -0.70504 & 2.96971 & -4.85502 \\
\hline $\mathrm{H}$ & -0.31908 & 4.00306 & -2.73468 \\
\hline $\mathrm{H}$ & -1.97949 & 4.70788 & -2.72258 \\
\hline $\mathrm{H}$ & -0.32213 & 5.70807 & 3.16909 \\
\hline $\mathrm{H}$ & 1.07152 & 5.53160 & 2.06588 \\
\hline $\mathrm{H}$ & 2.44254 & 4.59503 & 3.82573 \\
\hline $\mathrm{H}$ & 1.43546 & 5.74829 & 4.77709 \\
\hline $\mathrm{H}$ & 0.40331 & 3.90437 & 5.88709 \\
\hline $\mathrm{H}$ & 1.55372 & 2.83099 & 5.00896 \\
\hline $\mathrm{H}$ & -0.47995 & 2.02000 & 4.05604 \\
\hline
\end{tabular}




\begin{tabular}{|c|c|c|c|}
\hline $\mathrm{H}$ & -1.31028 & 3.57800 & 4.30776 \\
\hline $\mathrm{H}$ & -0.07128 & -5.00096 & -3.02603 \\
\hline $\mathrm{H}$ & 1.26781 & -3.79760 & -2.92903 \\
\hline $\mathrm{H}$ & 0.66106 & -2.85816 & -5.04075 \\
\hline $\mathrm{H}$ & -0.22877 & -4.40717 & -5.27708 \\
\hline $\mathrm{H}$ & -2.18973 & -3.03945 & -5.35022 \\
\hline & -1.28244 & -1.63991 & -4.66773 \\
\hline & -2.74327 & -2.23786 & -2.80430 \\
\hline $\mathrm{H}$ & -2.53866 & -3.98764 & -3.16426 \\
\hline $\mathrm{H}$ & -0.40803 & -3.68230 & 4.07930 \\
\hline $\mathrm{H}$ & -0.10899 & -1.94199 & 3.79701 \\
\hline $\mathrm{H}$ & 1.94459 & -1.99300 & 5.00566 \\
\hline $\mathrm{H}$ & 1.14397 & -3.38690 & 5.81933 \\
\hline r & 2.88561 & -4.75453 & 4.92800 \\
\hline $\mathrm{H}$ & 3.49883 & -3.34972 & 3.98001 \\
\hline $\mathrm{H}$ & 2.72319 & -4.78103 & 2.18892 \\
\hline $\mathrm{H}$ & 1.37566 & -5.39806 & 3.19381 \\
\hline $\mathrm{H}$ & -1.20230 & -1.61718 & 1.75487 \\
\hline $\mathrm{H}$ & -2.18759 & 1.35053 & 1.90909 \\
\hline $\mathrm{H}$ & -1.90104 & 0.41593 & 3.39753 \\
\hline $\mathrm{H}$ & 1.27835 & -0.58293 & 2.28875 \\
\hline $\mathrm{H}$ & 0.53575 & 1.10750 & 2.36614 \\
\hline $\mathrm{H}$ & -3.59426 & -1.24231 & 2.82839 \\
\hline $\mathrm{H}$ & -3.89489 & -0.48809 & 1.23448 \\
\hline $\mathrm{H}$ & -4.58439 & 1.66298 & 2.35500 \\
\hline $\mathrm{H}$ & -4.32306 & 0.90207 & 3.96402 \\
\hline $\mathrm{H}$ & -6.06424 & -0.84492 & 3.4175 \\
\hline $\mathrm{H}$ & -6.31521 & -0.09184 & 1.80291 \\
\hline $\mathrm{H}$ & -7.02999 & 2.06228 & 2.91677 \\
\hline $\mathrm{H}$ & -6.78569 & 1.30772 & 4.53141 \\
\hline $\mathrm{H}$ & -9.21418 & 1.23966 & 3.86416 \\
\hline $\mathrm{H}$ & -8.55525 & -0.43025 & 3.98749 \\
\hline $\mathrm{H}$ & -8.79940 & 0.32660 & 2.37015 \\
\hline & -2.77781 & -0.82880 & -1.19595 \\
\hline
\end{tabular}

\%eprnmr nuclei $=$ all Fe $\{$ rho, fgrad $\}$

end

n Calculated ${ }^{57} \mathrm{Fe}$ Mössbauer Properties: (dicPDI)Fe( $\left.\mathbf{N}_{2}\right)\left(\eta^{2}-1\right.$-octene)

$\rho(0)=11582.249904963 \times 10^{-3}-->0.447 \mathrm{~mm} / \mathrm{s}$

$\Delta \mathrm{E}=0.808 \mathrm{~mm} / \mathrm{s}$

- Optimized Coordinates - ( $\left.{ }^{\text {dic }} P D I\right) F e\left(N_{2}\right)\left(\eta^{2}-1-o c t e n e\right)$

$\begin{array}{lrrr}\mathrm{C} & 3.42061 & 1.79708 & 0.16894 \\ \mathrm{C} & 2.04934 & 1.54553 & 0.10286 \\ \mathrm{~N} & 1.56218 & 0.25286 & 0.02048 \\ \mathrm{C} & 2.46022 & -0.80088 & 0.01275 \\ \mathrm{C} & 3.83667 & -0.57379 & 0.07724\end{array}$




\begin{tabular}{|c|c|c|c|}
\hline C & 4.33094 & 0.73388 & 0.16027 \\
\hline C & 1.00012 & 2.52042 & 0.03350 \\
\hline C & 1.80498 & -2.07283 & -0.11087 \\
\hline C & 1.34118 & 3.97803 & -0.15521 \\
\hline$N$ & -0.22330 & 2.00857 & 0.04760 \\
\hline C & 2.61976 & -3.32613 & -0.31548 \\
\hline $\mathrm{N}$ & 0.48154 & -2.00841 & -0.08933 \\
\hline C & -1.30614 & 2.90975 & -0.26737 \\
\hline C & -0.26238 & -3.22021 & -0.33599 \\
\hline C & -1.62108 & 3.12096 & -1.63587 \\
\hline C & -2.65214 & 4.01920 & -1.95711 \\
\hline C & -3.35961 & 4.69432 & -0.96941 \\
\hline C & -3.03144 & 4.48755 & 0.36970 \\
\hline C & -2.00144 & 3.61391 & 0.75482 \\
\hline C & -0.67974 & -3.49283 & -1.66871 \\
\hline C & -1.38870 & -4.67746 & -1.91572 \\
\hline C & -1.68007 & -5.58130 & -0.89685 \\
\hline C & -1.25831 & -5.30779 & 0.39927 \\
\hline C & -0.54582 & -4.13744 & 0.71151 \\
\hline C & -0.81229 & 2.49754 & -2.76663 \\
\hline C & -1.64256 & 3.47633 & 2.23155 \\
\hline C & -0.06589 & -3.94351 & 2.14304 \\
\hline C & -0.30635 & -2.56895 & -2.81949 \\
\hline C & -1.65650 & 1.82329 & -3.89091 \\
\hline C & -0.92349 & 2.14289 & -5.20377 \\
\hline C & -0.42339 & 3.57566 & -4.97805 \\
\hline C & 0.08481 & 3.54722 & -3.52467 \\
\hline C & -0.33179 & 4.17392 & 2.70655 \\
\hline C & -0.50144 & 4.41771 & 4.23278 \\
\hline C & -1.93912 & 3.95866 & 4.57874 \\
\hline C & -2.68446 & 4.00218 & 3.24111 \\
\hline C & 1.11094 & -2.83894 & -3.42967 \\
\hline C & 1.00686 & -2.50896 & -4.94374 \\
\hline C & -0.40718 & -1.92542 & -5.15149 \\
\hline C & -1.24679 & -2.56830 & -4.04183 \\
\hline C & -1.11152 & -4.20930 & 3.25052 \\
\hline C & -0.28992 & -4.25916 & 4.55410 \\
\hline C & 1.14407 & -4.69423 & 4.12818 \\
\hline$C$ & 1.09235 & -4.86509 & 2.59504 \\
\hline $\mathrm{Fe}$ & -0.23760 & -0.07658 & 0.21211 \\
\hline$C$ & -1.34002 & -0.68672 & 1.98450 \\
\hline C & -2.67466 & -0.09917 & 2.38589 \\
\hline $\mathrm{N}$ & -1.86735 & -0.29406 & -0.65782 \\
\hline C & -0.09693 & -0.13993 & 2.27704 \\
\hline C & -2.84362 & -0.13257 & 3.91632 \\
\hline C & -4.19917 & 0.36198 & 4.43735 \\
\hline C & -4.18131 & 0.67308 & 5.93898 \\
\hline C & -5.50780 & 1.19539 & 6.50074 \\
\hline C & -5.40889 & 1.63682 & 7.96398 \\
\hline $\mathrm{H}$ & 3.77904 & 2.82634 & 0.22761 \\
\hline $\mathrm{H}$ & 4.52489 & -1.42072 & 0.05888 \\
\hline $\mathrm{H}$ & 5.40475 & 0.92353 & 0.21802 \\
\hline
\end{tabular}




\begin{tabular}{|c|c|c|c|}
\hline $\mathrm{H}$ & 0.44794 & 4.60721 & -0.21712 \\
\hline $\mathrm{H}$ & 1.92468 & 4.12170 & -1.07883 \\
\hline | & 1.95973 & 4.34860 & 0.67746 \\
\hline $\mathrm{H}$ & 1.98743 & -4.21276 & -0.43204 \\
\hline $\mathrm{H}$ & 3.29632 & -3.49940 & 0.53688 \\
\hline $\mathrm{H}$ & 3.25202 & -3.23829 & -1.21316 \\
\hline $\mathrm{H}$ & -2.89542 & 4.20341 & -3.00701 \\
\hline & -4.16232 & 5.38578 & -1.24008 \\
\hline & -3.58905 & 5.02936 & 1.13261 \\
\hline $\mathrm{H}$ & -1.71950 & -4.90943 & -2.92877 \\
\hline $\mathrm{H}$ & -2.23385 & -6.49720 & -1.11892 \\
\hline $\mathrm{H}$ & -1.48197 & -6.02450 & 1.19271 \\
\hline $\mathrm{H}$ & -0.15427 & 1.73560 & -2.33087 \\
\hline $\mathrm{H}$ & -1.51294 & 2.40370 & 2.43710 \\
\hline $\mathrm{H}$ & 0.28807 & -2.90913 & 2.25718 \\
\hline $\mathrm{H}$ & -0.29255 & -1.54182 & -2.42571 \\
\hline $\mathrm{H}$ & -2.66954 & 2.25409 & -3.92488 \\
\hline $\mathrm{H}$ & -1.78290 & 0.74473 & -3.71565 \\
\hline $\mathrm{H}$ & -0.05853 & 1.46803 & -5.33607 \\
\hline $\mathrm{H}$ & -1.56398 & 2.02890 & -6.09358 \\
\hline $\mathrm{H}$ & -1.26721 & 4.28224 & -5.07563 \\
\hline $\mathrm{H}$ & 0.34818 & 3.89619 & -5.69694 \\
\hline $\mathrm{H}$ & 1.13390 & 3.20640 & -3.50586 \\
\hline $\mathrm{H}$ & 0.06031 & 4.53947 & -3.04908 \\
\hline $\mathrm{H}$ & -0.20007 & 5.13018 & 2.17635 \\
\hline $\mathrm{H}$ & 0.55019 & 3.55724 & 2.48649 \\
\hline $\mathrm{H}$ & 0.24990 & 3.87344 & 4.82450 \\
\hline $\mathrm{H}$ & -0.36408 & 5.48503 & 4.4705 \\
\hline $\mathrm{H}$ & -2.41462 & 4.57690 & 5.35574 \\
\hline $\mathrm{H}$ & -1.92849 & 2.92324 & 4.95835 \\
\hline $\mathrm{H}$ & -3.61912 & 3.41957 & 3.23862 \\
\hline $\mathrm{H}$ & -2.95364 & 5.04688 & 3.00954 \\
\hline $\mathrm{H}$ & 1.40518 & -3.88966 & -3.27517 \\
\hline $\mathrm{H}$ & 1.86834 & -2.21952 & -2.92842 \\
\hline $\mathrm{H}$ & 1.79450 & -1.81641 & -5.2799 \\
\hline $\mathrm{H}$ & 1.12761 & -3.42744 & -5.54062 \\
\hline $\mathrm{H}$ & -0.80582 & -2.12664 & -6.15928 \\
\hline $\mathrm{H}$ & -0.39286 & -0.83020 & -5.0237 \\
\hline $\mathrm{H}$ & -2.19046 & -2.03804 & -3.8344 \\
\hline $\mathrm{H}$ & -1.51453 & -3.59273 & -4.3476 \\
\hline $\mathrm{H}$ & -1.60060 & -5.18184 & 3.0756 \\
\hline $\mathrm{H}$ & -1.91173 & -3.45380 & 3.27548 \\
\hline $\mathrm{H}$ & -0.26538 & -3.26387 & 5.02677 \\
\hline $\mathrm{H}$ & -0.73688 & -4.94285 & 5.2925 \\
\hline $\mathrm{H}$ & 1.47247 & -5.61772 & 4.6299 \\
\hline $\mathrm{H}$ & 1.87622 & -3.91772 & 4.4007 \\
\hline $\mathrm{H}$ & 2.04625 & -4.61798 & 2.1074 \\
\hline $\mathrm{H}$ & 0.84877 & -5.90764 & 2.3244 \\
\hline $\mathrm{H}$ & -1.37751 & -1.75952 & 1.8597 \\
\hline $\mathrm{H}$ & -3.48438 & -0.67376 & 1.9057 \\
\hline $\mathrm{H}$ & -2.79767 & 0.93578 & 2.0345 \\
\hline $\mathrm{H}$ & 0.75727 & -0.78172 & 2.4667 \\
\hline
\end{tabular}




$\begin{array}{rrrr}\mathrm{H} & -0.00712 & 0.85552 & 2.69699 \\ \mathrm{H} & -2.04137 & 0.48454 & 4.35794 \\ \mathrm{H} & -2.65320 & -1.15688 & 4.28577 \\ \mathrm{H} & -4.98360 & -0.38377 & 4.21290 \\ \mathrm{H} & -4.49376 & 1.27483 & 3.88714 \\ \mathrm{H} & -3.39250 & 1.42365 & 6.13346 \\ \mathrm{H} & -3.87078 & -0.22846 & 6.50126 \\ \mathrm{H} & -6.28340 & 0.41492 & 6.39299 \\ \mathrm{H} & -5.85510 & 2.04454 & 5.88333 \\ \mathrm{H} & -6.37449 & 2.00195 & 8.35087 \\ \mathrm{H} & -4.67649 & 2.45327 & 8.08544 \\ \mathrm{H} & -5.07981 & 0.80676 & 8.61269 \\ \mathrm{~N} & -2.84696 & -0.40279 & -1.16404\end{array}$

- DFT Input File - propylene

! RKS B3LYP RIJCOSX def2-SVP def2/J Normalprint SlowConv TightSCF Opt NumFreq Pal8 UCO

\%basis NewGTO 26 "def2-TZVP(-f)" end

NewGTO 7 "def2-TZVP(-f)" end

NewAuxGTO 26 "def2/J" end

NewAuxGTO 7 "def2/J" end

end

$\%$ SCF MaxIter 5000

TolE 1e-7

TolErr 1e-6

end

\%freq increment 0.01

restart true

centraldiff true

end

$\begin{array}{lrrr}{ }^{*} \text { xyz } 01 & & \\ \mathrm{C} & -3.57478 & 0.05046 & -0.03656 \\ \mathrm{C} & -2.47712 & 0.81081 & -0.13044 \\ \mathrm{H} & -3.51956 & -1.00395 & 0.21580 \\ \mathrm{H} & -4.55946 & 0.47282 & -0.21218 \\ \mathrm{C} & -1.08839 & 0.30983 & 0.09496 \\ \mathrm{H} & -2.58160 & 1.86296 & -0.38607 \\ \mathrm{H} & -1.06904 & -0.75529 & 0.34658 \\ \mathrm{H} & -0.62106 & 0.86315 & 0.91521 \\ \mathrm{H} & -0.48743 & 0.45863 & -0.80730 \\ { }_{*} & & & \end{array}$

end

- Calculated Thermochemical Properties - propylene (Calculated at 298.15 K)

Final Gibbs free enthalpy $\quad \ldots \quad-117.7358320526$ 
- Optimized Coordinates - propylene
C -3.59313479928057
0.05111946593362
$-0.03898490412634$
C -2.48429653273645
0.79167973867564
$-0.12389718138724$
H -3.55829855114300
$-1.01477178225382$
0.21415708697857
H $\quad-4.58098521923769$
0.48584411738197
$-0.21874875917898$
C -1.07931963109818
0.31208188138679
0.09577851900672
H -2.58837507234434
1.85397562715370
$-0.38347974886947$
H -1.04463673424219
$-0.75886911331690$
0.35237931325376
H -0.58872701759928
0.87535865616343
0.47300140887557
0.90857728270607
$-0.80578160838309$

\section{- DFT Input File - ( ${ }^{\text {dic } P D I) F e}\left(\mathrm{~N}_{2}\right)\left(\eta^{2}\right.$-propylene)}

! RKS B3LYP RIJCOSX def2-SVP def2/J Normalprint SlowConv TightSCF Opt NumFreq Pal8 UCO

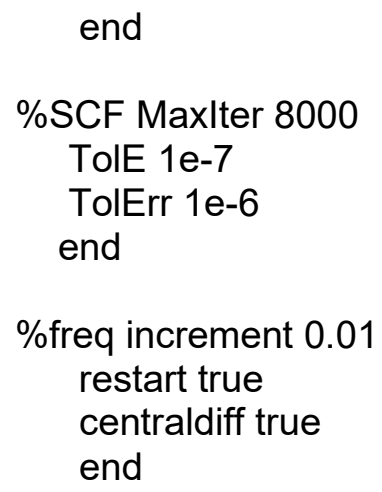

$\begin{array}{lrrr}{ }^{*} \text { xyz } 0 & 1 & & \\ \mathrm{C} & 1.16143 & 3.91887 & -0.43419 \\ \mathrm{C} & 1.17296 & 2.53918 & -0.22356 \\ \mathrm{~N} & -0.00000 & 1.83969 & 0.00000 \\ \mathrm{C} & -1.19994 & 2.53036 & 0.00409 \\ \mathrm{C} & -1.23634 & 3.91089 & -0.20360 \\ \mathrm{C} & -0.05002 & 4.61996 & -0.42959 \\ \mathrm{C} & 2.32800 & 1.69453 & -0.13128 \\ \mathrm{C} & -2.31903 & 1.67444 & 0.28251 \\ \mathrm{C} & 3.70531 & 2.30887 & -0.08460 \\ \mathrm{~N} & 2.05218 & 0.40452 & 0.00565 \\ \mathrm{C} & -3.68809 & 2.26842 & 0.50471 \\ \mathrm{~N} & -2.01141 & 0.38900 & 0.37804 \\ \mathrm{C} & 3.15513 & -0.46064 & 0.35042 \\ \mathrm{C} & -3.04803 & -0.53009 & 0.78227 \\ \mathrm{C} & 3.49865 & -0.58838 & 1.72238 \\ \mathrm{C} & 4.58946 & -1.40276 & 2.06883 \\ \mathrm{C} & 5.32686 & -2.07687 & 1.10257 \\ \mathrm{C} & 4.98691 & -1.93233 & -0.24176 \\ \mathrm{C} & 3.91680 & -1.12124 & -0.65339\end{array}$




\begin{tabular}{|c|c|c|c|}
\hline C & -3.16217 & -0.84839 & 2.16441 \\
\hline C & -4.17860 & -1.72838 & 2.56374 \\
\hline C & -5.06913 & -2.28141 & 1.64666 \\
\hline C & -4.95286 & -1.95483 & 0.30039 \\
\hline C & -3.95480 & -1.08075 & -0.16296 \\
\hline C & 2.80130 & 0.20901 & 2.81750 \\
\hline C & 3.63120 & -0.94860 & -2.14238 \\
\hline C & -3.93509 & -0.72561 & -1.64307 \\
\hline C & -2.25970 & -0.19779 & 3.20342 \\
\hline C & 2.36055 & -0.62051 & 4.06206 \\
\hline C & 2.61270 & 0.29058 & 5.27424 \\
\hline C & 3.91254 & 1.01289 & 4.89665 \\
\hline C & 3.70762 & 1.35358 & 3.40893 \\
\hline C & 4.04518 & 0.40917 & -2.78692 \\
\hline C & 4.22899 & 0.12788 & -4.30504 \\
\hline C & 4.02585 & -1.39685 & -4.48187 \\
\hline C & 4.28300 & -1.97889 & -3.08839 \\
\hline C & -2.75291 & 1.20396 & 3.69915 \\
\hline C & -2.32374 & 1.31894 & 5.18735 \\
\hline C & -1.47701 & 0.06172 & 5.47995 \\
\hline C & -2.01495 & -0.99039 & 4.50358 \\
\hline C & -4.06475 & -1.91152 & -2.62659 \\
\hline C & -4.34033 & -1.25245 & -3.99280 \\
\hline C & -5.00907 & 0.11688 & -3.66918 \\
\hline $\mathrm{C}$ & -5.07985 & 0.19539 & -2.12930 \\
\hline $\mathrm{Fe}$ & 0.00000 & 0.00000 & 0.00000 \\
\hline C & -0.49474 & -1.37238 & -1.61269 NewGTO "def2-TZVP(-f)" end \\
\hline C & 0.30652 & -2.61467 & -1.93253 \\
\hline $\mathrm{N}$ & 0.13867 & -1.54272 & 1.03002 \\
\hline C & -0.20574 & -0.08882 & -2.05852 NewGTO "def2-TZVP(-f)" end \\
\hline $\mathrm{H}$ & 0.21805 & -2.94536 & -3.43409 \\
\hline $\mathrm{H}$ & 2.10124 & 4.44772 & -0.60261 \\
\hline $\mathrm{H}$ & -2.19386 & 4.43443 & -0.18516 \\
\hline $\mathrm{H}$ & -0.06659 & 5.69842 & -0.59993 \\
\hline $\mathrm{H}$ & 4.49184 & 1.55412 & 0.01186 \\
\hline $\mathrm{H}$ & 3.79049 & 3.00164 & 0.76804 \\
\hline $\mathrm{H}$ & 3.90664 & 2.89362 & -0.99608 \\
\hline $\mathrm{H}$ & -4.43384 & 1.50318 & 0.74511 \\
\hline $\mathrm{H}$ & -4.03214 & 2.81049 & -0.39087 \\
\hline $\mathrm{H}$ & -3.66820 & 2.99620 & 1.33129 \\
\hline $\mathrm{H}$ & 4.87506 & -1.49841 & 3.11988 \\
\hline $\mathrm{H}$ & 6.16956 & -2.71015 & 1.39331 \\
\hline $\mathrm{H}$ & 5.57851 & -2.46029 & -0.98862 \\
\hline $\mathrm{H}$ & -4.28712 & -1.98802 & 3.61746 \\
\hline $\mathrm{H}$ & -5.85218 & -2.96406 & 1.98680 \\
\hline $\mathrm{H}$ & -5.65956 & -2.38463 & -0.41311 \\
\hline $\mathrm{H}$ & 1.90692 & 0.67092 & 2.38143 \\
\hline $\mathrm{H}$ & 2.54322 & -1.03528 & -2.27795 \\
\hline $\mathrm{H}$ & -2.99259 & -0.20599 & -1.86654 \\
\hline $\mathrm{H}$ & -1.27708 & -0.04138 & 2.73412 \\
\hline $\mathrm{H}$ & 2.97312 & -1.53089 & 4.15667 \\
\hline $\mathrm{H}$ & 1.31600 & -0.95557 & 3.98056 \\
\hline
\end{tabular}




\begin{tabular}{rrrr}
$\mathrm{H}$ & 1.79766 & 1.02971 & 5.37665 \\
$\mathrm{H}$ & 2.67040 & -0.26374 & 6.22523 \\
$\mathrm{H}$ & 4.76786 & 0.32407 & 5.01818 \\
$\mathrm{H}$ & 4.12466 & 1.90000 & 5.51544 \\
$\mathrm{H}$ & 3.17748 & 2.31692 & 3.31930 \\
$\mathrm{H}$ & 4.65847 & 1.45776 & 2.86454 \\
$\mathrm{H}$ & 4.98896 & 0.76451 & -2.34430 \\
$\mathrm{H}$ & 3.28904 & 1.18451 & -2.60330 \\
$\mathrm{H}$ & 3.52194 & 0.70382 & -4.92088 \\
$\mathrm{H}$ & 5.23698 & 0.42863 & -4.63376 \\
$\mathrm{H}$ & 4.67638 & -1.83226 & -5.25600 \\
$\mathrm{H}$ & 2.98656 & -1.61141 & -4.78212 \\
$\mathrm{H}$ & 3.88511 & -2.99735 & -2.95624 \\
$\mathrm{H}$ & 5.37096 & -2.03103 & -2.91205 \\
$\mathrm{H}$ & -3.84704 & 1.28757 & 3.59748 \\
$\mathrm{H}$ & -2.31438 & 2.00364 & 3.08531 \\
$\mathrm{H}$ & -1.77144 & 2.24854 & 5.39681 \\
$\mathrm{H}$ & -3.21320 & 1.33477 & 5.83778 \\
$\mathrm{H}$ & -1.54307 & -0.25933 & 6.53237 \\
$\mathrm{H}$ & -0.41256 & 0.25854 & 5.26964 \\
$\mathrm{H}$ & -1.33141 & -1.84023 & 4.34470 \\
$\mathrm{H}$ & -2.95279 & -1.40391 & 4.90872 \\
$\mathrm{H}$ & -4.91805 & -2.54593 & -2.33504 \\
$\mathrm{H}$ & -3.17651 & -2.56168 & -2.63442 \\
$\mathrm{H}$ & -3.39543 & -1.09945 & -4.53903 \\
$\mathrm{H}$ & -4.97006 & -1.88907 & -4.63360 \\
$\mathrm{H}$ & -6.00449 & 0.22052 & -4.12835 \\
$\mathrm{H}$ & -4.39874 & 0.94381 & -4.06536 \\
$\mathrm{H}$ & -4.98676 & 1.22363 & -1.75145 \\
$\mathrm{H}$ & -6.04195 & -0.20142 & -1.76004 \\
$\mathrm{H}$ & -1.53307 & -1.58817 & -1.40490 \\
$\mathrm{H}$ & -0.07951 & -3.45970 & -1.33816 \\
$\mathrm{H}$ & 1.36461 & -2.51307 & -1.64993 \\
$\mathrm{H}$ & -1.00467 & 0.61217 & -2.27730 \\
$\mathrm{~N}$ & 0.73020 & 0.13356 & -2.55842 \\
& 0.24264 & -2.46782 & 1.63093 \\
& & & \\
\hline
\end{tabular}

\%eprnmr nuclei $=$ all Fe $\{$ rho, fgrad $\}$

end

a Calculated ${ }^{57} \mathrm{Fe}$ Mössbauer Properties: ( ${ }^{\text {dic } P D I) F e}\left(\mathbf{N}_{2}\right)\left(\eta^{2}\right.$-propylene)

$\rho(0)=11582.195512533 \times 10^{-3}-->0.461 \mathrm{~mm} / \mathrm{s}$

$\Delta \mathrm{E}=0.703 \mathrm{~mm} / \mathrm{s}$

- Calculated Thermochemical Properties - ( ${ }^{\text {dic }}$ PDI)Fe( $\left.\mathrm{N}_{2}\right)\left(\eta^{2}\right.$-propylene) (Calculated at 298.15 K)

Final Gibbs free enthalpy $\quad \ldots \quad-3246.0366912427$ 
- Optimized Coordinates - ( ${ }^{\text {dic }}$ PDI)Fe $\left(\mathrm{N}_{2}\right)\left(\eta^{2}\right.$-propylene $)$

\begin{tabular}{|c|c|c|c|}
\hline & .15969485527493 & & \\
\hline & 1.17387196960750 & & -0.17188670013477 \\
\hline & 916 & 16 & 980 \\
\hline & & 34 & \\
\hline & | 24959488697290 & & \\
\hline & .05867964771455 & 4.68143640446173 & -0.37624405419373 \\
\hline & 32650253806872 & 1.75903872634573 & -0.04909238746922 \\
\hline & -2.32633101330066 & 1.70332323125942 & 3109 \\
\hline & & & \\
\hline & & & \\
\hline & -3.71 & 847061691808 & \\
\hline & $-1.9 \varsigma$ & 0910046 & 0. \\
\hline & 3.11570882150466 & 55236279350 & \\
\hline & -3.00965294203218 & 575062575759 & 75583 \\
\hline & & & \\
\hline & & & \\
\hline & 5.14 & -2.2 & \\
\hline & & -2.00 & \\
\hline & & -1.08 & \\
\hline & -3.10 & 43344 & 4573 \\
\hline & -4.0 & & \\
\hline & & & \\
\hline & & & \\
\hline & & & \\
\hline & & & \\
\hline & 3.58 & -0.7 & -2.1 \\
\hline & -3.9 & -0.8 & -1. \\
\hline & -2.2 & & \\
\hline & & & \\
\hline & & & \\
\hline & & & \\
\hline & 3.77 & & \\
\hline & 4.15 & & \\
\hline & 4.32 & & \\
\hline & & & \\
\hline & & & \\
\hline & -2.631751 & & \\
\hline & -2.1662049 & & \\
\hline & -1.5 & & \\
\hline & -2.1 & -0.7 & \\
\hline & & & \\
\hline & -4.506 & & 94054 \\
\hline & -5.07579821090123 & -0.11725058189095 & -3.66785808445267 \\
\hline & -5.21404194582388 & -0.07239928378846 & -2.14142330314619 \\
\hline & & & \\
\hline & & 228 & \\
\hline & & -2.61 & \\
\hline & 0.14180466619317 & -1.47905309613850 & 17594224922 \\
\hline & -0.20922957886984 & -0.13078617120378 & -2.08444145533640 \\
\hline & -0.01726714212904 & -3.20989284062293 & -2.6684565280856 \\
\hline
\end{tabular}



H 2.10170437076012
4.53347424462444
$-0.43379520551123$
H -2.21123374406557
4.47521275681746
$-0.25719979029812$
H $\quad-0.07949466475510$
5.76387788945412
$-0.51680546388294$
1.59049655787318
0.09972120684427
H 3.78419624195901
2.93479306505876
H 3.92072637990974
3.05419819612843
H -4.44779206391440
1.49856043825727
2.73407607034360
H -4.04622453287817
3.05534113026000
H -3.74909619655210
H 4.72830138270634
$-1.79225093846657$
H 5.92992755418232
$-2.99316566327625$
H 5.39312344064333
$-2.52762762922566$
H -4.13965486594414
H $\quad-5.64930297343252$
H $\quad-5.52568953326614$
H 1.93177170789491
$-1.98080992562989$
$-3.08545113481210$
$-2.52550215232190$
0.63545332280108
1.02636115888384
$-0.73511091562302$
0.59072555838672
$-0.62571657965093$
1.08943346973396
3.04399972496519
1.24825547012391
$-1.11880845566728$
3.63306352835613
2.03739529938659
H 2.49658690535229
$-0.72629919354937$
H $\quad-3.08538891477729$
$-0.14569281413966$
$-0.04107438443313$
H -1.20500298449470
H 2.85791255137146
$-1.73479737916116$
$-0.37222555846337$
2.45010340167455
$-2.30138041404812$
$-1.89645505175028$
2.77226909698546
H 1.24423226497520
$-1.04514861469829$
4.07736939564007
H 1.86593311417679
H 2.66480939005282
0.80909206119491
3.96069065746307
$-0.58856202720327$
5.47848741247288
H 4.78345135835510
$-0.04628569523793$
6.22512350357247
H 4.23742704069244
1.52902140131106
5.03719699060958
H 3.30021758620432
2.13980679144698
5.63792042775309
3.48448179474083
1.22836619375399
0.78692399969644
2.95787159260887
H 5.12654676203184
H 3.48462389133602
1.40444382640120
1.09487220910558
0.56152278566093
H 5.37292148391145
H 4.49996977723520
H 2.85729789859813
$-1.53605439058426$
$-1.13348506934919$
H 3.60145005942601
$-2.77546764638105$
H 5.18815220649669
$-1.99082784630496$
H $\quad-3.72387667009496$
1.51365399661584
H -2.17302314283324
H -1.47485768509694
H -3.03030919834341
H -1.65161364753090
H -0.41561107939136
H -1.56493635411704
H -3.13870035019740
H -4.49064770654402
H -2.86932205380037
H -3.76277332236940
H $\quad-5.30400553939156$
H -6.02123688249030
H -4.35797517706653
H -5.32150222856162
H -6.10342149982549
H -1.46995234938564
2.10175326573891
2.51558996813310
1.90936455915554
0.16305734582034
0.38516066931131
$-1.68145523898288$
$-0.96593317001763$
$-2.84022038083709$
$-2.40961612110176$
$-1.50686516418162$
$-2.21315946890376$
0.08793241716206
0.65584227675518
0.94680361306904
$-0.64182168782577$
$-1.65586987106322$
$-2.23206051709652$
$-2.47067263511648$
$-4.81606207358828$
$-4.53679943482427$
$-5.35418730489017$
$-4.82796706164728$
$-3.14076603957869$
$-3.03703796824255$
3.43124929789038
2.81333142268698
5.04881046912171
5.61607805234625
6.53096519946163
5.27862408413649
4.56657302197797
4.96800740636431
$-2.20716863483350$
$-2.74421189982667$
$-4.77358962415663$
$-4.29098608063117$
$-4.19420369416799$
$-3.99035369812896$
$-1.74120087151221$
$-1.81755556693220$
$-1.34852701485971$ 

H $\quad 0.42338222242129-3.28505864642181 \quad-0.95774685590033$
H $1.44018623959031-2.37294650234591 \quad-2.10065042648456$
$\begin{array}{llll}\mathrm{H} & -1.02997938315227 & 0.54180495002675 & -2.30402992291785\end{array}$
H $0.71551529625917 \quad 0.10381680076450 \quad-2.59719098462300$
N $0.24188372534347 \quad-2.41157356494690 \quad 1.60667859425831$

\section{- DFT Input File - (dicPDI)Fe( $\left.\mathbf{N}_{2}\right)\left(\eta^{2}\right.$-propylene) (triplet state)}

! UKS B3LYP RIJCOSX def2-SVP def2/J Normalprint SlowConv TightSCF Opt NumFreq Pal8 UCO

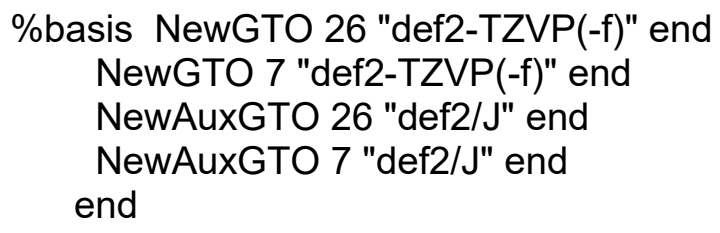

$\begin{array}{lrrr}\mathrm{C} & 1.17285 & 3.93019 & -0.32053 \\ \mathrm{C} & 1.18032 & 2.54298 & -0.16791 \\ \mathrm{~N} & 0.00000 & 1.83870 & -0.00000 \\ \mathrm{C} & -1.20099 & 2.52859 & 0.00486 \\ \mathrm{C} & -1.23317 & 3.91539 & -0.15874 \\ \mathrm{C} & -0.04061 & 4.63031 & -0.32268 \\ \mathrm{C} & 2.32901 & 1.68687 & -0.08271 \\ \mathrm{C} & -2.31638 & 1.66546 & 0.26944 \\ \mathrm{C} & 3.71426 & 2.27403 & 0.02445 \\ \mathrm{~N} & 2.03675 & 0.39562 & 0.00051 \\ \mathrm{C} & -3.69891 & 2.24024 & 0.45025 \\ \mathrm{~N} & -1.99240 & 0.38520 & 0.39694 \\ \mathrm{C} & 3.11082 & -0.52696 & 0.27347 \\ \mathrm{C} & -3.00165 & -0.54292 & 0.84942 \\ \mathrm{C} & 3.46996 & -0.77688 & 1.62419 \\ \mathrm{C} & 4.48921 & -1.70882 & 1.88311 \\ \mathrm{C} & 5.14322 & -2.37625 & 0.85366 \\ \mathrm{C} & 4.80102 & -2.09794 & -0.46899 \\ \mathrm{C} & 3.80209 & -1.16689 & -0.79288 \\ \mathrm{C} & -3.06141 & -0.83706 & 2.24463 \\ \mathrm{C} & -4.01069 & -1.77205 & 2.68380 \\ \mathrm{C} & -4.89789 & -2.38840 & 1.80331 \\ \mathrm{C} & -4.85094 & -2.06581 & 0.45323 \\ \mathrm{C} & -3.91606 & -1.14737 & -0.05601 \\ \mathrm{C} & 2.86249 & -0.00709 & 2.79155\end{array}$




\begin{tabular}{|c|c|c|c|}
\hline C & 3.51100 & -0.84347 & -2.25402 \\
\hline C & -3.99294 & -0.81024 & -1.54168 \\
\hline C & -2.15037 & -0.14349 & 3.25602 \\
\hline C & 2.40017 & -0.88759 & 3.99376 \\
\hline C & 2.76245 & -0.08874 & 5.25544 \\
\hline C & 4.09608 & 0.57059 & 4.87916 \\
\hline C & 3.86521 & 1.03080 & 3.42693 \\
\hline C & 4.07789 & 0.50407 & -2.79796 \\
\hline C & 4.20582 & 0.32758 & -4.33746 \\
\hline C & 3.77981 & -1.13089 & -4.63233 \\
\hline C & 4.00226 & -1.86144 & -3.30358 \\
\hline C & -2.53521 & 1.33175 & 3.61216 \\
\hline C & -2.02880 & 1.58180 & 5.06250 \\
\hline C & -1.36419 & 0.26110 & 5.50971 \\
\hline C & -2.02547 & -0.81275 & 4.64064 \\
\hline C & -3.98241 & -2.02071 & -2.51786 \\
\hline C & -4.63139 & -1.51591 & -3.83662 \\
\hline C & -5.18183 & -0.10206 & -3.51739 \\
\hline $\mathrm{C}$ & -5.27899 & -0.06866 & -1.98749 \\
\hline $\mathrm{Fe}$ & 0.00000 & 0.00000 & 0.00000 \\
\hline C & -0.51282 & -1.44454 & -1.58793 NewGTO "def2-TZVP(-f)" end \\
\hline C & 0.32675 & -2.66134 & -1.85158 \\
\hline $\mathrm{N}$ & 0.14764 & -1.54276 & 1.01361 \\
\hline C & -0.27506 & -0.16642 & -2.06465 NewGTO "def2-TZVP(-f)" end \\
\hline $\mathrm{H}$ & -0.12334 & -3.24204 & -2.67818 \\
\hline $\mathrm{H}$ & 2.11622 & 4.46603 & -0.43908 \\
\hline $\mathrm{H}$ & -2.19081 & 4.43986 & -0.14784 \\
\hline $\mathrm{H}$ & -0.05651 & 5.71399 & -0.45397 \\
\hline $\mathrm{H}$ & 4.48975 & 1.50033 & 0.00705 \\
\hline $\mathrm{H}$ & 3.82419 & 2.84239 & 0.96280 \\
\hline $\mathrm{H}$ & 3.91469 & 2.97518 & -0.80068 \\
\hline $\mathrm{H}$ & -4.42770 & 1.47383 & 0.73569 \\
\hline $\mathrm{H}$ & -4.04882 & 2.71612 & -0.48113 \\
\hline $\mathrm{H}$ & -3.70355 & 3.02099 & 1.22799 \\
\hline $\mathrm{H}$ & 4.78391 & -1.90824 & 2.91666 \\
\hline $\mathrm{H}$ & 5.92763 & -3.10369 & 1.07992 \\
\hline $\mathrm{H}$ & 5.33169 & -2.61459 & -1.26816 \\
\hline $\mathrm{H}$ & -4.06643 & -2.03267 & 3.74080 \\
\hline $\mathrm{H}$ & -5.62684 & -3.11242 & 2.17724 \\
\hline $\mathrm{H}$ & -5.56299 & -2.53370 & -0.23027 \\
\hline $\mathrm{H}$ & 1.99204 & 0.54592 & 2.41708 \\
\hline $\mathrm{H}$ & 2.41917 & -0.78116 & -2.35853 \\
\hline $\mathrm{H}$ & -3.14522 & -0.16051 & -1.80005 \\
\hline $\mathrm{H}$ & -1.14035 & -0.10882 & 2.81748 \\
\hline $\mathrm{H}$ & 2.94223 & -1.84473 & 4.00003 \\
\hline $\mathrm{H}$ & 1.33158 & -1.14162 & 3.93201 \\
\hline $\mathrm{H}$ & 2.00829 & 0.69522 & 5.44743 \\
\hline $\mathrm{H}$ & 2.81558 & -0.71468 & 6.16127 \\
\hline $\mathrm{H}$ & 4.90609 & -0.17917 & 4.92156 \\
\hline $\mathrm{H}$ & 4.38887 & 1.39535 & 5.54923 \\
\hline $\mathrm{H}$ & 3.39953 & 2.03105 & 3.42654 \\
\hline $\mathrm{H}$ & 4.80161 & 1.11292 & 2.85482 \\
\hline
\end{tabular}




\begin{tabular}{rrrr}
$\mathrm{H}$ & 5.06205 & 0.71095 & -2.34737 \\
$\mathrm{H}$ & 3.41934 & 1.34316 & -2.53711 \\
$\mathrm{H}$ & 3.58773 & 1.05103 & -4.89029 \\
$\mathrm{H}$ & 5.24513 & 0.50256 & -4.65959 \\
$\mathrm{H}$ & 4.33397 & -1.58139 & -5.47012 \\
$\mathrm{H}$ & 2.70917 & -1.17040 & -4.89702 \\
$\mathrm{H}$ & 3.48486 & -2.83190 & -3.24347 \\
$\mathrm{H}$ & 5.07999 & -2.06050 & -3.17588 \\
$\mathrm{H}$ & -3.62827 & 1.45999 & 3.55590 \\
$\mathrm{H}$ & -2.08978 & 2.04106 & 2.90150 \\
$\mathrm{H}$ & -1.32902 & 2.43111 & 5.12078 \\
$\mathrm{H}$ & -2.87360 & 1.83236 & 5.72452 \\
$\mathrm{H}$ & -1.48502 & 0.06794 & 6.58827 \\
$\mathrm{H}$ & -0.28108 & 0.29069 & 5.30519 \\
$\mathrm{H}$ & -1.46545 & -1.76102 & 4.60759 \\
$\mathrm{H}$ & -3.02219 & -1.03660 & 5.05661 \\
$\mathrm{H}$ & -4.57975 & -2.84139 & -2.09466 \\
$\mathrm{H}$ & -2.96992 & -2.41901 & -2.67135 \\
$\mathrm{H}$ & -3.90987 & -1.49273 & -4.66886 \\
$\mathrm{H}$ & -5.44329 & -2.19095 & -4.15093 \\
$\mathrm{H}$ & -6.13928 & 0.11478 & -4.01664 \\
$\mathrm{H}$ & -4.46669 & 0.66805 & -3.85275 \\
$\mathrm{H}$ & -5.36760 & 0.94803 & -1.57641 \\
$\mathrm{H}$ & -6.16395 & -0.63379 & -1.64455 \\
$\mathrm{H}$ & -1.52788 & -1.68765 & -1.30755 \\
$\mathrm{H}$ & 0.36211 & -3.33466 & -0.98050 \\
$\mathrm{H}$ & 1.35536 & -2.42113 & -2.14283 \\
$\mathrm{H}$ & -1.09597 & 0.51431 & -2.25686 \\
$\mathrm{H}$ & 0.63754 & 0.06518 & -2.60002 \\
$\mathrm{~N}$ & 0.25598 & -2.48085 & 1.59475 \\
$*$ & & & \\
\hline & & &
\end{tabular}

\%eprnmr nuclei $=$ all Fe $\{$ rho, fgrad $\}$

end

n Calculated ${ }^{57} \mathrm{Fe}$ Mössbauer Properties: (dicPDI)Fe( $\left.\mathrm{N}_{2}\right)\left(\mathrm{n}^{2}\right.$-propylene) (triplet state)

$\rho(0)=11581.483221477 \times 10^{-3}-->0.676 \mathrm{~mm} / \mathrm{s}$

$\Delta \mathrm{E}=0.984 \mathrm{~mm} / \mathrm{s}$

- Calculated Thermochemical Properties - (dicPDI)Fe( $\left.\mathbf{N}_{2}\right)\left(\eta^{2}\right.$-propylene) (triplet state) (Calculated at 298.15 K)

Final Gibbs free enthalpy $\quad$.. $\quad-3246.0205959614$

- Optimized Coordinates - ( ${ }^{\text {dic } P D I) F e}\left(\mathrm{~N}_{2}\right)\left(\eta^{2}\right.$-propylene) (triplet state)
C 1.15969485527493
3.99108215693539
$-0.33603286921380$
C 1.17387196960750
2.60523731834519
$-0.17188670013477$
N -0.00499746314916
1.89291672944316
$-0.02971168255980$
C -1.21101738451225
2.57319524149134
$-0.06250610273133$ 


\begin{tabular}{|c|c|c|c|}
\hline & -1.24959488697290 & 95834984932965 & \\
\hline & -0.05867964771455 & 4.68143640446173 & -0.37624405419373 \\
\hline & 2.32650253806872 & 1.75903872634573 & -0.04909238746922 \\
\hline & -2.32633101330066 & 1.70332323125942 & 3109 \\
\hline & 3.70379051386006 & 2.35808808049997 & 3570872 \\
\hline & 2.04217481231412 & 0.46620306239522 & 0.03689525529778 \\
\hline & -3.71762369153814 & 2.26847061691808 & 0.31852694084418 \\
\hline & -1.99592597474026 & 0.42676030910046 & 0.32591502440036 \\
\hline & 3.11570882150466 & -0.44555236279350 & 0.34593778421384 \\
\hline & & -0.50575062575759 & \\
\hline & 3.4405 & 27776 & \\
\hline & 0224296 & 08413 & \\
\hline & 9228321 & 968070 & \\
\hline & 731247799 & -2.00886021536405 & -0.33831 \\
\hline & 001583452 & -1.08843583911255 & -0.6963 \\
\hline & -3.10436089870932 & 1543344 & \\
\hline & -4.0 & 851 & \\
\hline & -4.9 & & \\
\hline & 38717103 & 146852 & 6928318 \\
\hline & 10013240 & 71291012384 & -0.16553219500953 \\
\hline & 2.79 & & \\
\hline & 3.58 & $-0.77 \subseteq$ & -2.16731 \\
\hline & & -0.8 & \\
\hline & -2.2 & & \\
\hline & 2.30 & -0.7 & \\
\hline & 2.63 & & \\
\hline & & & \\
\hline & 02625507 & 75654 & 3.505 \\
\hline & 4.1 & 0.5 & -2.7 \\
\hline & 4.3 & & \\
\hline & 3.9 & -1.08 & -4.5 \\
\hline & 4.112 & 081 & -3.195 \\
\hline & 175105597096 & 535847 & \\
\hline & 99321685 & 328197 & 4.97 \\
\hline & -1.5 & 363 & \\
\hline & -2.1 & -0.7 & \\
\hline & -3.8 & -2.0 & -2. \\
\hline & -4.5 & 277 & 494054 \\
\hline & -5.07 & 8189095 & 445267 \\
\hline & 04194582388 & 239928378846 & -2.14142330314619 \\
\hline & 0.00 & 2921639056 & -0.0 \\
\hline & & -1.4 & \\
\hline , & & -2.6 & -1.83 \\
\hline $\mathrm{N}$ & 0.14180466619317 & -1.47905309613850 & 1.01517594224922 \\
\hline & -0.20922957886984 & -0.13078617120378 & -2.08444145533640 \\
\hline & -0.01726714212904 & -3.20989284062293 & -2.66845652808569 \\
\hline & 2.10170437076012 & 4.53347424462444 & -0.43379520551123 \\
\hline & -2.21123 & & -0.25719979029812 \\
\hline & -0.07949466475510 & 5.76387788945412 & -0.5168054638829 \\
\hline & 4.48546130764754 & 1.59049655787318 & 0.09972120684427 \\
\hline $\mathrm{H}$ & 3.78419624195901 & 2.93479306505876 & 1.02636115888384 \\
\hline & 3.92072637990974 & 3.05419819612843 & -0.73511091562302 \\
\hline
\end{tabular}




\begin{tabular}{|c|c|c|c|}
\hline & -4.44779206391440 & & \\
\hline & -4.04622453287817 & & \\
\hline & -3.74909619655210 & 3.05534113026000 & 3396 \\
\hline & 4.72830138270634 & -1.79225093846657 & \\
\hline & .92992755418232 & -2.99316566327625 & 1.24825547012391 \\
\hline & 5.39312344064333 & -2.52762762922566 & $-1.1188084556672 \varepsilon$ \\
\hline & -4.13965486594414 & -1.98080992562989 & 3.6330635283561 \\
\hline & & & \\
\hline & & & \\
\hline & 1.93177170789491 & 0.63545332280108 & 2.450103401 \\
\hline & 2.49658690535229 & 29919354937 & -2.301 \\
\hline & -3.08538891477729 & 569281413966 & \\
\hline & -1.20500298449470 & 107438443313 & \\
\hline & 91255137146 & -1.73 & 4.077369395640 \\
\hline & & -1.0 & \\
\hline & 1.86 & & \\
\hline & 282 & -0.5 & \\
\hline & 4.78345 & $-0.0<$ & \\
\hline & & & \\
\hline & & & \\
\hline & & & \\
\hline & & & \\
\hline & 3.48 & & -2.47 \\
\hline & & & -4.816 \\
\hline & & & \\
\hline & 4.49 & -1.5 & -5.3 \\
\hline & & & \\
\hline & & -2.7 & \\
\hline & & & \\
\hline & & & \\
\hline & -2.1730231 & 3891 & 2.8 \\
\hline & -1.47 & & \\
\hline & & & \\
\hline & & & \\
\hline & & & \\
\hline & & & \\
\hline & -3.13 & & \\
\hline & $-4.4 \mathrm{c}$ & -2.8 & -2.2 \\
\hline & -2.8 & -2.4 & -2.7 \\
\hline & & & \\
\hline & & -2.2 & \\
\hline & -6.021 & & -4.19 \\
\hline & -4.3579751 & 75518 & \\
\hline & -5.3215022 & 0.9 & -1.7 \\
\hline & $-6.103421<$ & 8782577 & \\
\hline & $-1.46 s$ & & \\
\hline & 8222242129 & & \\
\hline & & -2.372 & -2.10065042648456 \\
\hline & -1.02997938315227 & 0.54180495002675 & -2.3040299229178 \\
\hline & 0.71551529625917 & 0.10381680076450 & -2.59719098462300 \\
\hline & 0.24188372534347 & 57356494690 & 160667859425831 \\
\hline
\end{tabular}


- DFT Input File - (dicPDI)Fe( $\eta^{2}$-propylene)

! UKS B3LYP RIJCOSX def2-SVP def2/J Normalprint SlowConv TightSCF Opt Pal8 UCO

\%basis NewGTO 26 "def2-TZVP(-f)" end

NewGTO 7 "def2-TZVP(-f)" end

NewAuxGTO 26 "def2/J" end

NewAuxGTO 7 "def2/J" end

end

$\%$ SCF BrokenSym 1,1

Maxlter 5000

TolE $1 \mathrm{e}-7$

TolErr 1e-6

end

* xyz 03

$\begin{array}{lrrr}\mathrm{C} & -1.04259 & 3.87927 & 0.06525 \\ \mathrm{C} & -1.09950 & 2.54031 & 0.04171 \\ \mathrm{~N} & 0.00000 & 1.86119 & -0.00000 \\ \mathrm{C} & 1.19391 & 2.37954 & -0.00835 \\ \mathrm{C} & 1.28814 & 3.72020 & 0.00885 \\ \mathrm{C} & 0.16772 & 4.46375 & 0.04216 \\ \mathrm{C} & -2.14921 & 1.70861 & 0.07278 \\ \mathrm{C} & 2.19769 & 1.47133 & -0.01126 \\ \mathrm{C} & 3.63668 & 1.93393 & 0.09729 \\ \mathrm{~N} & 1.89320 & 0.18447 & 0.00063 \\ \mathrm{C} & -3.55702 & 2.24564 & 0.20312 \\ \mathrm{~N} & -1.84940 & 0.42520 & 0.05326 \\ \mathrm{C} & 2.85255 & -0.69324 & -0.21432 \\ \mathrm{C} & -2.86991 & -0.39053 & -0.13342 \\ \mathrm{C} & -3.49968 & -0.46253 & -1.34342 \\ \mathrm{C} & -4.45134 & -1.39595 & -1.53697 \\ \mathrm{C} & -4.84631 & -2.22683 & -0.57044 \\ \mathrm{C} & -4.31553 & -2.06824 & 0.64328 \\ \mathrm{C} & -3.34415 & -1.16796 & 0.88035 \\ \mathrm{C} & 3.52969 & -0.77628 & -1.39863 \\ \mathrm{C} & 4.45042 & -1.74641 & -1.56754 \\ \mathrm{C} & 4.75771 & -2.62552 & -0.61175 \\ \mathrm{C} & 4.18288 & -2.46742 & 0.58101 \\ \mathrm{C} & 3.26122 & -1.51084 & 0.79523 \\ \mathrm{C} & -3.25924 & 0.44274 & -2.55802 \\ \mathrm{C} & -3.03666 & -0.92656 & 2.35265 \\ \mathrm{C} & 2.94715 & -1.28096 & 2.26715 \\ \mathrm{C} & 3.40142 & 0.16264 & -2.60546 \\ \mathrm{C} & -2.88116 & -0.31685 & -3.85502 \\ \mathrm{C} & -4.01263 & -0.07341 & -4.86455 \\ \mathrm{C} & -4.50372 & 1.32643 & -4.48447 \\ \mathrm{C} & -4.51079 & 1.26957 & -2.95100 \\ \mathrm{C} & -4.13362 & -0.11828 & 3.07068 \\ \mathrm{C} & -3.80991 & -0.25527 & 4.57028 \\ \mathrm{C} & -2.82767 & -1.43497 & 4.68910\end{array}$




\begin{tabular}{|c|c|c|c|}
\hline C & -2.86580 & -2.11950 & 3.31353 \\
\hline C & 2.50742 & -2.49671 & 3.11365 \\
\hline C & 3.41955 & -2.50584 & 4.35174 \\
\hline C & 3.88668 & -1.05082 & 4.48363 \\
\hline C & 4.14361 & -0.66222 & 3.02218 \\
\hline C & 4.73880 & 0.87997 & -2.93448 \\
\hline C & 5.13329 & 0.46217 & -4.35844 \\
\hline C & 3.77947 & 0.21137 & -5.02769 \\
\hline C & 2.99936 & -0.52882 & -3.93305 \\
\hline $\mathrm{Fe}$ & 0.00000 & 0.00000 & 0.00000 \\
\hline C & -0.04698 & -1.78194 & 0.83739 NewGTO "def2-TZVP(-f)" end \\
\hline $\mathrm{H}$ & -1.95312 & 4.49693 & 0.10536 \\
\hline $\mathrm{H}$ & 2.26479 & 4.22919 & 0.00350 \\
\hline $\mathrm{H}$ & 0.24173 & 5.56421 & 0.06171 \\
\hline $\mathrm{H}$ & 3.75604 & 2.54548 & 1.02073 \\
\hline $\mathrm{H}$ & 4.39962 & 1.13344 & 0.18927 \\
\hline $\mathrm{H}$ & 3.91625 & 2.57160 & -0.76964 \\
\hline $\mathrm{H}$ & -3.65709 & 2.75437 & 1.18883 \\
\hline $\mathrm{H}$ & -3.76000 & 3.00981 & -0.57826 \\
\hline $\mathrm{H}$ & -4.37570 & 1.49814 & 0.15740 \\
\hline $\mathrm{H}$ & -4.95858 & -1.49481 & -2.50957 \\
\hline $\mathrm{H}$ & -5.64961 & -2.96107 & -0.74827 \\
\hline $\mathrm{H}$ & -4.75046 & -2.67533 & 1.45108 \\
\hline $\mathrm{H}$ & 5.01222 & -1.83709 & -2.51086 \\
\hline $\mathrm{H}$ & 5.53381 & -3.39233 & -0.77146 \\
\hline $\mathrm{H}$ & 4.54194 & -3.10759 & 1.40337 \\
\hline $\mathrm{H}$ & -2.47245 & 1.20257 & -2.35002 \\
\hline $\mathrm{H}$ & -2.12395 & -0.31048 & 2.43650 \\
\hline $\mathrm{H}$ & 2.16013 & -0.51153 & 2.36286 \\
\hline $\mathrm{H}$ & 2.67944 & 0.98708 & -2.41107 \\
\hline $\mathrm{H}$ & -1.95902 & 0.12863 & -4.30162 \\
\hline $\mathrm{H}$ & -2.67716 & -1.39928 & -3.69617 \\
\hline $\mathrm{H}$ & -4.83251 & -0.81504 & -4.71991 \\
\hline $\mathrm{H}$ & -3.67331 & -0.13675 & -5.92358 \\
\hline $\mathrm{H}$ & -3.77107 & 2.09299 & -4.83320 \\
\hline $\mathrm{H}$ & -5.50208 & 1.56615 & -4.91593 \\
\hline $\mathrm{H}$ & -5.45255 & 0.77365 & -2.62217 \\
\hline $\mathrm{H}$ & -4.52276 & 2.29360 & -2.51718 \\
\hline $\mathrm{H}$ & -4.16941 & 0.94443 & 2.74240 \\
\hline $\mathrm{H}$ & -5.13275 & -0.57004 & 2.86468 \\
\hline $\mathrm{H}$ & -4.74000 & -0.44127 & 5.15664 \\
\hline $\mathrm{H}$ & -3.34117 & 0.67778 & 4.96302 \\
\hline $\mathrm{H}$ & -1.79549 & -1.05199 & 4.87741 \\
\hline $\mathrm{H}$ & -3.08923 & -2.13157 & 5.51806 \\
\hline $\mathrm{H}$ & -3.74436 & -2.80311 & 3.29891 \\
\hline $\mathrm{H}$ & -1.95856 & -2.73438 & 3.12915 \\
\hline $\mathrm{H}$ & 1.45026 & -2.36626 & 3.44905 \\
\hline $\mathrm{H}$ & 2.55174 & -3.46557 & 2.56856 \\
\hline $\mathrm{H}$ & 4.30431 & -3.15945 & 4.16101 \\
\hline $\mathrm{H}$ & 2.90578 & -2.87592 & 5.26808 \\
\hline $\mathrm{H}$ & 3.06678 & -0.42296 & 4.90687 \\
\hline $\mathrm{H}$ & 4.78943 & -0.94236 & 5.12655 \\
\hline
\end{tabular}




$\begin{array}{lrrr}\mathrm{H} & 5.10795 & -1.11908 & 2.69926 \\ \mathrm{H} & 4.22830 & 0.43910 & 2.87687 \\ \mathrm{H} & 4.58620 & 1.98670 & -2.93986 \\ \mathrm{H} & 5.55193 & 0.67603 & -2.20241 \\ \mathrm{H} & 5.72270 & -0.48448 & -4.33546 \\ \mathrm{H} & 5.73831 & 1.23347 & -4.88707 \\ \mathrm{H} & 3.28335 & 1.18499 & -5.25534 \\ \mathrm{H} & 3.86616 & -0.37343 & -5.97152 \\ \mathrm{H} & 3.30172 & -1.60057 & -3.94704 \\ \mathrm{H} & 1.91075 & -0.51209 & -4.15584 \\ \mathrm{H} & -1.41482 & -1.86689 & -1.12611 \\ \mathrm{H} & -0.90606 & -1.95855 & 1.46155 \\ \mathrm{H} & 0.92091 & -2.17068 & 1.10405 \\ \mathrm{C} & -0.38013 & -1.76309 & -0.81565 \text { NewGTO "def2-TZVP(-f)" end } \\ \mathrm{C} & 0.62909 & -2.57980 & -1.59620 \\ \mathrm{H} & 0.36297 & -3.65568 & -1.48468 \\ \mathrm{H} & 1.66962 & -2.48641 & -1.24210 \\ \mathrm{H} & 0.58872 & -2.35190 & -2.68407 \\ * & & & \end{array}$

$\%$ plots format cube $\operatorname{dim} 1100 \operatorname{dim} 2100 \operatorname{dim} 3100$ SpinDens("dicPDIFepropylene_BS11_trip.cube");

end

n Calculated Thermochemical Properties - ( ${ }^{\text {dic } P D I) F e(~} \eta^{2}$-propylene) (Calculated at $\left.298.15 \mathrm{~K}\right)$

Final Gibbs free enthalpy ... -3136.6349028513

Final Gibbs free enthalpy ... $\quad-3136.6315550259$ (singlet state)

- Mulliken Spin Density Plot - (dicPDI)Fe( $\eta^{2}$-propylene)

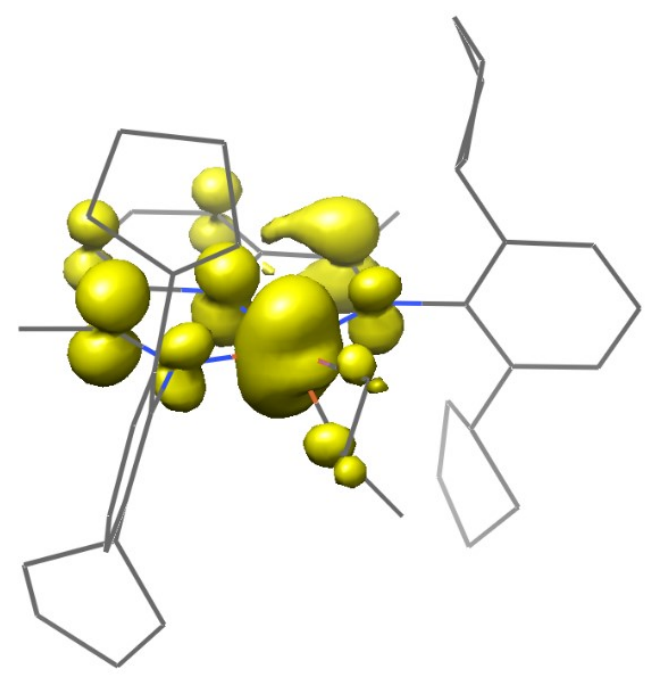




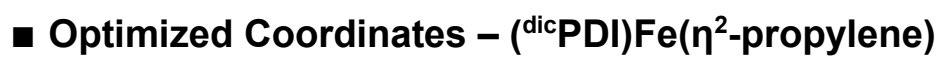

\begin{tabular}{|c|c|c|c|}
\hline & -0.99360933679595 & & \\
\hline & & 57956032538007 & 15724528670862 \\
\hline & 5169614931 & 65043685951 & \\
\hline & 606222021192 & 2.49447828234520 & \\
\hline & & 3.89038041758178 & \\
\hline & 24910543150771 & 4.61985897885871 & 0.37199615380097 \\
\hline & 2.17262363362987 & 1.71737288165580 & 0.14685257049571 \\
\hline & 2.42758754790579 & 32855834 & \\
\hline & & & \\
\hline & & & \\
\hline & 312 & 2.26 & \\
\hline & 04360650 & 25570 & -0.0 \\
\hline & 3.06134097770875 & -0.73700182072100 & \\
\hline & -2.98012947805468 & -0.51579751017600 & \\
\hline & & 4770 & \\
\hline & & & \\
\hline & & -2.4 & \\
\hline & & -2.2 & \\
\hline & & -1.278562 & 0.97562 \\
\hline & & -1.1269712 & -1.39814 \\
\hline & & -2.1 & \\
\hline & & & \\
\hline & & & \\
\hline & & & \\
\hline & & & -2.6 \\
\hline & -2.7 & -1.0 & \\
\hline & 2.79 & -0.8 & 223 \\
\hline & & -0. & \\
\hline & & & \\
\hline & & & \\
\hline & & & \\
\hline & -4.4 & & \\
\hline & -3.7 & -0.2 & \\
\hline & & & \\
\hline & & & \\
\hline & & & 872 \\
\hline & & & \\
\hline & & -1.87 & \\
\hline & 3.12 & -0.4 & \\
\hline & & -0.2 & \\
\hline & & & \\
\hline & & & \\
\hline & 3.47835792947835 & -0.24655411028602 & 8715836337 \\
\hline & 3.73349758285056 & -1.25166448154121 & 9124347894 \\
\hline & & & -0.28 \\
\hline & & -2.09 & \\
\hline & & & 878224 \\
\hline & & & \\
\hline 11 & 0.29554280272766 & 5.70208362096056 & 0.52028687371989 \\
\hline & 3.99642231323060 & 2.34490316521536 & 1.2306052402517 \\
\hline
\end{tabular}



H 4.57119024208056
1.19825462903246
2.84929644434873
2.79346053627064
3.01360224303077
1.48483388751516
$-1.80203025391284$
$-3.17162700272275$
$-2.81533101068644$
$-2.47909041470047$
$-3.54391093321977$
$-2.80795113115028$
0.81036457086012
$-0.45104517143400$
$-0.10544739187361$
$-0.32403916131984$
$-0.96974580998396$
$-1.47186075334499$
$-0.05568816172708$
0.72560591116986
2.44768708548283
2.02710353968714
0.52180297975973
1.90879728270328
0.82403905899154
$-0.53612450474401$
$-1.14112876497273$
0.15631426011092
$-1.25575678464822$
$-2.52633724218640$
$-3.02137741983369$
$-2.82885810438810$
$-1.87303276658187$
$-3.01267097464863$
$-2.61154171128396$
$-2.06071332415853$
0.28759352524225
$-0.32054521948216$
$-0.86639118969136$
0.75496807292439
1.72555293023440
0.96275810373929
1.43652648231070
1.91512281626678
$-0.23150856946423$
$-0.50772105768390$
$-1.52412071970516$
$-2.18792919365407$
$-1.81599583212881$
$-2.21711500103517$
$-2.46988300569681$
$-1.90865694445784$
$-2.29782782014423$
0.00007653237539
$-0.46044161680339$
1.36393171396603
$-0.37427873799881$
0.39488850014738
$-2.44783237950058$
$-0.49166025180004$
1.68993864618397
$-2.23929939431785$
$-0.09196449046581$
2.00421958809380
$-2.37333956214875$
2.22657170035583
2.14349545772374
$-2.73699257643289$
$-3.92770658977094$
$-4.02584342837306$
$-5.85905796756086$
$-5.58550617409207$
$-4.27676415329059$
$-5.05915712220211$
$-3.19292885668136$
$-2.35215800185344$
3.16656637061374
3.11933678204883
5.28392323892449
5.38095105984495
4.65158621944762
5.36687793872363
3.18192792727529
2.77378868912649
3.10038429147266
2.77598792119571
4.85766152223436
5.37619786884748
4.85356490141975
5.59015922759456
3.36226687864626
3.13598238648478
$-2.47630003969705$
$-2.65895196400172$
$-4.94770391926001$
$-4.83069812918312$
$-5.37811335009602$
$-6.06213943520513$
$-3.98966579922884$
$-4.11401692752788$
$-1.49057726291597$
0.79208002975873
0.24287092166398
$-1.29391006568655$
$-2.49267351446320$ 

H $0.05519640913995 \quad-3.23242014088406 \quad-2.91602317957447$
H $1.51938491484858-2.48553496443131 \quad-2.24390132756796$
H $0.41889287736254-1.54689329773043 \quad-3.29843030515265$

- DFT Input File - (dicPDI)Fe( $\eta^{2}$-propylene $)_{2}$

! UKS B3LYP RIJCOSX def2-SVP def2/J Normalprint SlowConv TightSCF Opt Pal8 UCO

\%basis NewGTO 26 "def2-TZVP(-f)" end

NewGTO 7 "def2-TZVP(-f)" end

NewAuxGTO 26 "def2/J" end

NewAuxGTO 7 "def2/J" end

end

\%SCF BrokenSym 3,1

Maxiter 500

TolE 1e-7

TolErr 1e-6

end

${ }^{*}$ xyz 03

$\begin{array}{lrrr}\text { C } & 1.89825 & -3.10634 & 2.47934 \\ \text { C } & 1.61642 & -2.13554 & 1.47881 \\ \text { N } & 0.37221 & -2.10916 & 0.84823 \\ \text { C } & -0.50595 & -3.11701 & 1.12210 \\ \text { C } & -0.23283 & -4.11660 & 2.05489 \\ \text { C } & 0.98188 & -4.08963 & 2.77764 \\ \text { C } & 2.60322 & -1.18698 & 1.04890 \\ \text { C } & -1.82353 & -3.15557 & 0.40944 \\ \text { C } & 3.98637 & -1.22098 & 1.66129 \\ \text { N } & 2.24393 & -0.28639 & 0.12622 \\ \text { C } & -2.57422 & -4.47304 & 0.39844 \\ \text { N } & -2.25349 & -2.09376 & -0.15795 \\ \text { C } & 3.24041 & 0.41558 & -0.62018 \\ \text { C } & -3.51355 & -1.98526 & -0.80191 \\ \text { C } & 3.53464 & 1.77919 & -0.34541 \\ \text { C } & 4.42582 & 2.46267 & -1.18847 \\ \text { C } & 5.02689 & 1.83379 & -2.27638 \\ \text { C } & 4.73822 & 0.49581 & -2.53877 \\ \text { C } & 3.86223 & -0.24124 & -1.72565 \\ \text { C } & -3.55411 & -2.00113 & -2.22579 \\ \text { C } & -4.77663 & -1.74139 & -2.86153 \\ \text { C } & -5.93641 & -1.48067 & -2.13382 \\ \text { C } & -5.88326 & -1.46329 & -0.74214 \\ \text { C } & -4.68964 & -1.71292 & -0.04371 \\ \text { C } & 2.87492 & 2.49138 & 0.82602 \\ \text { C } & 3.58664 & -1.70977 & -2.03413 \\ \text { C } & -4.67469 & -1.68326 & 1.48241 \\ \text { C } & -2.30048 & -2.31383 & -3.03353 \\ \text { Fe } & 0.11168 & -0.16532 & -0.02729 \\ \text { C } & -1.20872 & 0.85062 & -1.35967 \text { NewGTO "def2-TZVP(-f)" end }\end{array}$




\begin{tabular}{|c|c|c|c|}
\hline C & -0.07700 & 0.58632 & -2.10732 NewGTO "def2-TZVP(-f)" end \\
\hline C & -1.39074 & 0.52067 & 1.84085 NewGTO "def2-TZVP(-f)" end \\
\hline C & -0.12239 & 0.98514 & 1.96885 NewGTO "def2-TZVP(-f)" end \\
\hline C & 0.84520 & 1.67568 & -2.58518 \\
\hline C & -2.56540 & 1.37974 & 1.49883 \\
\hline $\mathrm{H}$ & 2.85342 & -3.07934 & 3.00230 \\
\hline $\mathrm{H}$ & -0.95531 & -4.90796 & 2.24719 \\
\hline $\mathrm{H}$ & 1.19205 & -4.83753 & 3.54578 \\
\hline $\mathrm{H}$ & 3.95828 & -0.88839 & 2.71481 \\
\hline $\mathrm{H}$ & 4.43280 & -2.22716 & 1.65447 \\
\hline $\mathrm{H}$ & 4.66740 & -0.54986 & 1.12407 \\
\hline $\mathrm{H}$ & -1.92117 & -5.28528 & 0.04423 \\
\hline $\mathrm{H}$ & -2.92224 & -4.74814 & 1.40570 \\
\hline $\mathrm{H}$ & -3.45407 & -4.40917 & -0.25360 \\
\hline $\mathrm{H}$ & 5.70477 & 2.38946 & -2.92988 \\
\hline $\mathrm{H}$ & 5.18993 & 0.01947 & -3.41125 \\
\hline $\mathrm{H}$ & -4.82308 & -1.72695 & -3.95230 \\
\hline $\mathrm{H}$ & -6.87569 & -1.27248 & -2.65283 \\
\hline $\mathrm{H}$ & -6.79252 & -1.23535 & -0.18335 \\
\hline C & 3.79365 & 2.67763 & 2.06662 \\
\hline C & 2.41134 & 3.94972 & 0.53473 \\
\hline $\mathrm{H}$ & 2.01814 & 1.87576 & 1.10955 \\
\hline C & 3.54539 & -2.07139 & -3.54838 \\
\hline C & 4.69881 & -2.66127 & -1.50198 \\
\hline $\mathrm{H}$ & 2.62739 & -1.96441 & -1.56526 \\
\hline C & -5.47950 & -0.51033 & 2.12342 \\
\hline $\mathrm{H}$ & -1.43954 & -2.04369 & -2.40716 \\
\hline C & -2.18948 & -3.81378 & -3.43397 \\
\hline C & -2.21470 & -1.59507 & -4.40946 \\
\hline $\mathrm{H}$ & -2.06462 & 0.18870 & -1.37926 \\
\hline $\mathrm{H}$ & -1.40386 & 1.86785 & -1.03472 \\
\hline $\mathrm{H}$ & -0.03921 & -0.33679 & -2.67672 \\
\hline $\mathrm{H}$ & -1.61286 & -0.49087 & 2.16145 \\
\hline $\mathrm{H}$ & 0.08827 & 2.04548 & 1.80816 \\
\hline $\mathrm{H}$ & 0.65539 & 0.40259 & 2.46532 \\
\hline $\mathrm{H}$ & 1.86049 & 1.31088 & -2.78838 \\
\hline $\mathrm{H}$ & 0.91698 & 2.50188 & -1.86202 \\
\hline $\mathrm{H}$ & 0.45330 & 2.10411 & -3.52696 \\
\hline $\mathrm{H}$ & -3.18717 & 0.93576 & 0.71955 \\
\hline $\mathrm{H}$ & -2.25280 & 2.37328 & 1.17352 \\
\hline $\mathrm{H}$ & -3.20164 & 1.50058 & 2.38242 \\
\hline $\mathrm{H}$ & -1.16416 & -1.32977 & -4.60773 \\
\hline $\mathrm{H}$ & -2.77271 & -0.64804 & -4.41270 \\
\hline C & -2.71804 & -2.59916 & -5.48732 \\
\hline $\mathrm{H}$ & -1.12674 & -4.05342 & -3.61731 \\
\hline C & -2.97942 & -3.93320 & -4.74480 \\
\hline $\mathrm{H}$ & -2.55114 & -4.49910 & -2.65168 \\
\hline $\mathrm{H}$ & -1.95678 & -2.73198 & -6.27266 \\
\hline $\mathrm{H}$ & -3.62460 & -2.23822 & -5.99932 \\
\hline $\mathrm{H}$ & -4.05265 & -4.04889 & -4.51926 \\
\hline $\mathrm{H}$ & -2.68063 & -4.81356 & -5.33663 \\
\hline $\mathrm{H}$ & -5.56853 & 0.34712 & 1.44108 \\
\hline
\end{tabular}




$\begin{array}{lrrr}\mathrm{H} & -4.92955 & -0.14819 & 3.00615 \\ \mathrm{C} & -6.86277 & -1.07058 & 2.56344 \\ \mathrm{H} & -3.62548 & -1.61787 & 1.79613 \\ \mathrm{C} & -5.32417 & -2.93597 & 2.14274 \\ \mathrm{C} & -6.81599 & -2.59454 & 2.29950 \\ \mathrm{H} & -4.86509 & -3.08697 & 3.13629 \\ \mathrm{H} & -5.16135 & -3.85808 & 1.56544 \\ \mathrm{H} & -7.69054 & -0.58796 & 2.01927 \\ \mathrm{H} & -7.03962 & -0.85993 & 3.63052 \\ \mathrm{H} & -7.29683 & -3.17905 & 3.09931 \\ \mathrm{H} & -7.35324 & -2.84329 & 1.37022 \\ \mathrm{H} & 4.26021 & -3.66127 & -1.33783 \\ \mathrm{H} & 5.12439 & -2.33312 & -0.54449 \\ \mathrm{C} & 5.75070 & -2.71921 & -2.62007 \\ \mathrm{H} & 3.24209 & -1.21612 & -4.17092 \\ \mathrm{H} & 2.78037 & -2.84941 & -3.69826 \\ \mathrm{C} & 4.94338 & -2.63876 & -3.93642 \\ \mathrm{H} & 5.45582 & -2.01207 & -4.68392 \\ \mathrm{H} & 4.84142 & -3.63585 & -4.39438 \\ \mathrm{H} & 6.38143 & -3.62088 & -2.55785 \\ \mathrm{H} & 6.42984 & -1.85511 & -2.53229 \\ \mathrm{H} & 2.23056 & 4.11825 & -0.53792 \\ \mathrm{C} & 3.48969 & 4.90674 & 1.12212 \\ \mathrm{H} & 1.44740 & 4.12536 & 1.04103 \\ \mathrm{H} & 4.47022 & 1.82664 & 2.24199 \\ \mathrm{H} & 3.15123 & 2.77495 & 2.96095 \\ \mathrm{C} & 4.53356 & 4.00085 & 1.81722 \\ \mathrm{H} & 4.64873 & 3.51424 & -0.99838 \\ \mathrm{H} & 3.02935 & 5.59897 & 1.84593 \\ \mathrm{H} & 3.95153 & 5.53639 & 0.34405 \\ \mathrm{H} & 5.39442 & 3.82476 & 1.15236 \\ \mathrm{H} & 4.93696 & 4.44568 & 2.74111 \\ * & & & \\ & & & \end{array}$

$\%$ plots format cube

$\operatorname{dim} 1100 \operatorname{dim} 2100 \operatorname{dim} 3100$

SpinDens("MePDIFepropylene2.cube");

end

n Calculated ${ }^{57}$ Fe Mössbauer Properties: (dicPDI)Fe( $\eta^{2}$-propylene) ${ }_{2}$

$\rho(0)=11580.97688638010^{-3}-->0.829 \mathrm{~mm} / \mathrm{s}$

$\Delta \mathrm{E}=1.197 \mathrm{~mm} / \mathrm{s}$

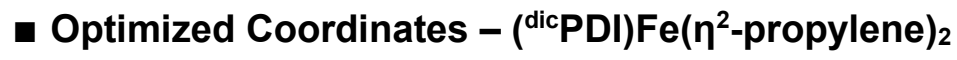
C 2.04906118095994
$-3.34543063157234$
2.28850671270492
C $1.68504753013546-2.26784769587569$
1.44132023617942
N 0.37848678101247
$-2.14901266829578$
0.97622537218103
C -0.49036112279445
$-3.17546655969326$
1.20869514744366
C -0.15191305332183
$-4.26450137885363$
2.00679399712364
C 1.13299617674724
$-4.33081095224076$
2.59523091174765 


\begin{tabular}{|c|c|c|c|}
\hline & 2.63083269237839 & -1.29092053722106 & \\
\hline & & -3.14523737856595 & 50696339806650 \\
\hline & 205 & 4740594 & \\
\hline & 2.15972617482528 & -0.29890015118819 & 0.220689445 \\
\hline & -2.61730670074439 & -4.42904168563293 & 0.48332099560342 \\
\hline & -2.16383191968791 & -2.06648457836516 & -0.08751975991276 \\
\hline & 3.03789041996487 & 0.45842804509538 & -0.60600960239306 \\
\hline & -3.32743931968094 & -1.97905168299185 & 102231 \\
\hline & 984404 & 1.825 & \\
\hline & 135489 & & \\
\hline & 264764 & 3277161974 & -2.3203 \\
\hline & 617593 & 0.63860056931694 & -2.622 \\
\hline & 3.56038395599178 & -0.13775588284507 & -1.7937558305155 \\
\hline & -3.22654176344486 & -2.30090134360280 & -2.279190816043 \\
\hline & 959 & 928 & \\
\hline & 2291 & -1.7 & \\
\hline & 5031 & -1.3 & \\
\hline & -4.53 & 060 & -0.3 \\
\hline & 906847378 & 2.4867 & 0.899 \\
\hline & 97410 & -1.5556 & -2.20 \\
\hline & 927 & -1.0 & \\
\hline & 774 & -2.7 & \\
\hline & & -0.1 & \\
\hline & 6484 & 79065 & $-0.5 c$ \\
\hline & 48446 & 1875181 & -1.5304 \\
\hline & 01213 & 3390462 & 2.3970 \\
\hline & 0.27 & & $2.7 \subseteq$ \\
\hline & 0.3 & & \\
\hline & -2.0 & 58 & \\
\hline & 5629402825 & -3.35 & \\
\hline & 82998725791 & 3369 & 1030 \\
\hline & 9081710741 & -5.16 & \\
\hline & 4.20 & -1.18 & 2.45 \\
\hline & & -2.3 & \\
\hline & 4.69 & -0.6 & \\
\hline & -2.02 & & 6991 \\
\hline & & -4.7 & 96503 \\
\hline & -3.52819284794615 & 21032482 & 30611215 \\
\hline & 5.32600157012417 & 2.55 & -2.98 \\
\hline & 4.78 & 0.2 & -3.5 \\
\hline & & & \\
\hline & & & \\
\hline & -6.58548895115589 & -0.95125328399740 & 550101977 \\
\hline & 3.63859223637820 & 2.61945309370053 & 317897144 \\
\hline & 2.19625566983130 & 3.95 & 0.68 \\
\hline & & & \\
\hline & 2.882 & $-1.722 \varepsilon$ & -3.7 \\
\hline & 4.27090907848221 & -2.62788919086352 & 91683 \\
\hline $\mathrm{H}$ & 2.27099169767499 & -1.81681154313144 & -1.63494497661664 \\
\hline & -5.59381739507432 & 0.12222653705523 & 1.40322611919775 \\
\hline & -1.1140238485261 & -2.47293801179644 & -2.17668343145037 \\
\hline & & & \\
\hline
\end{tabular}


C -1.60175776079596

H -2.30893848306096

H -1.17371692924960

H -0.96276894812520

H -1.41096605109955

H 0.63222991670679

H 0.94133298755532

H 1.21393963646145

H 0.80386680507722

H -0.15247131900949

H -2.80385813765256

H -1.55334591035850

H -2.55750174749758

H -0.58705504971451

H -2.28130190456312

C -1.72313079938571

H -0.75973052515785

C -2.28282484078328

H -2.39389552116546

H -0.73090862456874

H -2.35550983764283

H -3.38560270600660

H -1.93879965761930

H -5.69347811092689

H $\quad-5.18292439247382$

C -6.94765412054771

H -3.59686193983676

C -5.15410245471291

C -6.68238351321065

H -4.80954304915249

H -4.79197186203339

H $\quad-7.75348414976108$

H $\quad-7.28196728978068$

H $\quad-7.19028077462137$

H -7.05133465832584

H 3.77858941094088

H 4.86013018292251

C 5.13516913075105

H 2.59266814908492

H 2.02392941858712

C 4.14498061064088

H 4.58664898655307

H 3.88029128577345

H 5.70050881856319

H 5.88295536883466

H 1.96762271104112

C 3.29821794981190

H 1.25566432769765

H 4.32268527157789

H 3.04073020641512

C 4.36666519078496

H 4.33547215177360
$-2.13570187295593$

0.76983137892786

2.21701896011372

$-0.24917203726220$

$-0.66850096176200$

1.55826786463599

$-0.25910279250585$

0.86480527262515

2.29769478228196

1.89042503813119

1.39622917771932

2.43823147693681

1.40192423508472

$-1.70732132540951$

$-1.29839294725951$

$-3.27340177344197$

$-4.60976319996968$

$-4.49590902419450$

$-4.88096777038058$

$-3.51341896337444$

$-2.98836569193010$

$-4.50751142952453$

$-5.45204988132288$

0.80805796133281

0.71722041090814

$-0.51159330375923$

$-0.77447521383810$

$-2.16027690426265$

$-2.03176922114625$

$-1.94724069001181$

$-3.16466619121288$

$-0.30064032843591$

$-0.08653213886560$

$-2.48761579472651$

$-2.55894753847167$

$-3.60890406867530$

$-2.44143833357647$

$-2.62460083811612$

$-0.77120337986853$

$-2.40331249389934$

$-2.35461343305923$

$-1.70094277978410$

$-3.29300264851580$

$-3.56151534685116$

$-1.81662353487247$

4.16366825371148

4.88671028817555

4.10290319750331

1.76229166151976

2.67095041256033

3.95448156350365

3.60506229914449
$-4.28632925166488$

$-0.25986253965058$

$-0.19644649363560$

$-2.00554626723323$

2.43276573364812

2.97411975361397

3.03927814841096

$-2.74550128442145$

$-1.78359541484003$

$-3.22400929324261$

1.69747257335061

2.41255711555006

3.45021441699814

$-4.29562321313272$

$-4.50078973508096$

$-5.33783069050139$

$-3.03525537380597$

$-4.58185907364420$

$-2.40840692574512$

$-5.75390507910970$

$-6.19404869232839$

$-4.62336166290893$

$-5.00879707942750$

0.54885547842535

2.23328412124195

1.83692663351015

1.41606533974302

2.04277583327789

1.96169217980886

3.07127338250738

1.77834601352578

1.11536667062807

2.79643616511956

2.82582280235108

1.06670929705205

$-1.83286485451572$

$-1.04994876486413$

$-3.22912843155194$

$-4.19911192133131$

$-3.85507160810684$

$-4.38559728681191$

$-5.15522813456538$

$-4.89898463733138$

$-3.36103113709318$

$-3.16554087858235$

$-0.37278246013920$

1.26242028253157

1.23824309841258

2.21123575415448

3.03661170534717

1.88302216412229

$-0.98049038138496$ 

H 2.86889695945847
5.55374307031206
2.02772839586307
H 3.73218602092824
5.54373257383914
0.49109135597194
H 5.20060703456292
3.80526901079397
1.17839514474895
H 4.80539472376244
4.36486994941874
2.80661999993456

n DFT Input File - (cyclomet-dicPDI)Fe-H

! UKS B3LYP RIJCOSX def2-SVP def2/J Normalprint SlowConv TightSCF NumFreq Opt Pal8 UCO
\%basis NewGTO 26 "def2-TZVP(-f)" end NewGTO 7 "def2-TZVP(-f)" end NewAuxGTO 26 "def2/J" end NewAuxGTO 7 "def2/J" end end
\%SCF BrokenSym 3,1
Maxiter 5000
TolE 1e-7
TolErr 1e-6
end
\%freq increment 0.01
restart true
centraldiff true
end

$\begin{array}{lrrr}{ }^{*} \text { xyz } 03 & & & \\ \text { C } & 3.94579 & -1.18029 & 0.40718 \\ \text { C } & 2.55947 & -1.17859 & 0.24036 \\ \text { N } & 1.91385 & -0.00000 & -0.00000 \\ \text { C } & 2.55008 & 1.20799 & 0.06674 \\ \text { C } & 3.94113 & 1.24648 & 0.21564 \\ \text { C } & 4.64044 & 0.04207 & 0.35447 \\ \text { C } & 1.67899 & -2.33506 & 0.26329 \\ \text { C } & 1.63035 & 2.32009 & 0.08782 \\ \text { C } & 2.12629 & 3.72276 & 0.33470 \\ \text { N } & 0.34887 & 1.97269 & -0.00132 \\ \text { C } & 2.22251 & -3.68704 & 0.66421 \\ \text { N } & 0.39562 & -2.12588 & -0.05575 \\ \text { C } & -0.36893 & -3.28106 & -0.43833 \\ \text { C } & -0.68719 & 2.90568 & 0.32948 \\ \text { C } & 0.10159 & -4.11667 & -1.50519 \\ \text { C } & -0.53918 & -5.34207 & -1.74309 \\ \text { C } & -1.66108 & -5.73530 & -1.02297 \\ \text { C } & -2.19504 & -4.85408 & -0.08990 \\ \text { C } & -1.59435 & -3.62139 & 0.21048 \\ \text { C } & -1.37510 & 2.67397 & 1.55311 \\ \text { C } & -2.34813 & 3.59784 & 1.95179 \\ \text { C } & -2.66130 & 4.71172 & 1.17358 \\ \text { C } & -2.01627 & 4.89529 & -0.04633 \\ \text { C } & -1.03130 & 4.00272 & -0.50091 \\ \text { C } & 1.19559 & -3.72893 & -2.50108\end{array}$




\begin{tabular}{|c|c|c|c|}
\hline C & -2.33438 & -2.71015 & 1.17387 \\
\hline C & -0.44012 & 4.21350 & -1.88794 \\
\hline C & -1.16696 & 1.43256 & 2.40410 \\
\hline C & 2.37012 & -4.76400 & -2.66683 \\
\hline C & 2.28739 & -5.29922 & -4.10925 \\
\hline C & 1.69737 & -4.12418 & -4.89810 \\
\hline C & 0.61860 & -3.58026 & -3.95160 \\
\hline C & -3.71016 & -2.22918 & 0.62552 \\
\hline C & -4.58737 & -1.90627 & 1.86459 \\
\hline C & -3.77050 & -2.35618 & 3.10207 \\
\hline C & -2.69742 & -3.30984 & 2.55398 \\
\hline C & -1.47582 & 4.06287 & -3.03227 \\
\hline C & -0.76100 & 4.67155 & -4.24005 \\
\hline C & -0.14011 & 5.95575 & -3.65668 \\
\hline C & 0.21760 & 5.60524 & -2.18278 \\
\hline C & -0.04761 & 0.46633 & 2.02924 NewGTO "def2-TZVP(-f)" end \\
\hline C & -0.08644 & -0.58236 & 3.13973 \\
\hline C & -0.49495 & 0.22711 & 4.41688 \\
\hline C & -0.88727 & 1.64348 & 3.91623 \\
\hline $\mathrm{Fe}$ & 0.00000 & 0.00000 & 0.00000 \\
\hline $\mathrm{H}$ & 4.47957 & -2.11439 & 0.59079 \\
\hline $\mathrm{H}$ & 4.47126 & 2.20043 & 0.23972 \\
\hline $\mathrm{H}$ & 5.72795 & 0.05782 & 0.46216 \\
\hline $\mathrm{H}$ & 1.30080 & 4.41619 & 0.52785 \\
\hline $\mathrm{H}$ & 2.69544 & 4.10694 & -0.52910 \\
\hline $\mathrm{H}$ & 2.80664 & 3.75173 & 1.20073 \\
\hline $\mathrm{H}$ & 2.87029 & -4.13729 & -0.10375 \\
\hline $\mathrm{H}$ & 1.40622 & -4.39107 & 0.86370 \\
\hline $\mathrm{H}$ & 2.82955 & -3.59262 & 1.57883 \\
\hline $\mathrm{H}$ & -0.16113 & -5.99258 & -2.53642 \\
\hline $\mathrm{H}$ & -2.14162 & -6.69749 & -1.21809 \\
\hline $\mathrm{H}$ & -3.11673 & -5.13184 & 0.42791 \\
\hline $\mathrm{H}$ & -2.88112 & 3.42931 & 2.89106 \\
\hline $\mathrm{H}$ & -3.42106 & 5.42389 & 1.50666 \\
\hline $\mathrm{H}$ & -2.28894 & 5.74971 & -0.67178 \\
\hline $\mathrm{H}$ & 1.62794 & -2.76810 & -2.19804 \\
\hline $\mathrm{H}$ & -1.72436 & -1.81424 & 1.33223 \\
\hline $\mathrm{H}$ & 0.33170 & 3.44690 & -2.05179 \\
\hline $\mathrm{H}$ & -2.11898 & 0.87212 & 2.33144 \\
\hline $\mathrm{H}$ & 2.32998 & -5.56894 & -1.91808 \\
\hline $\mathrm{H}$ & 3.33327 & -4.24616 & -2.52445 \\
\hline $\mathrm{H}$ & 3.26005 & -5.64775 & -4.49288 \\
\hline $\mathrm{H}$ & 1.59852 & -6.16069 & -4.16313 \\
\hline $\mathrm{H}$ & 1.29097 & -4.41595 & -5.88051 \\
\hline $\mathrm{H}$ & 2.47621 & -3.36180 & -5.07787 \\
\hline $\mathrm{H}$ & 0.32218 & -2.54608 & -4.17943 \\
\hline $\mathrm{H}$ & -0.29353 & -4.18857 & -4.05242 \\
\hline $\mathrm{H}$ & -4.17724 & -3.02451 & 0.02270 \\
\hline $\mathrm{H}$ & -3.57649 & -1.36223 & -0.03957 \\
\hline $\mathrm{H}$ & -4.83599 & -0.83461 & 1.92219 \\
\hline $\mathrm{H}$ & -5.54690 & -2.44390 & 1.80855 \\
\hline $\mathrm{H}$ & -4.39564 & -2.82655 & 3.87855 \\
\hline
\end{tabular}




$\begin{array}{lrrr}H & -3.28826 & -1.48333 & 3.57267 \\ H & -1.82249 & -3.40591 & 3.21775 \\ H & -3.12351 & -4.32135 & 2.43362 \\ H & -2.38014 & 4.64986 & -2.79283 \\ H & -1.80387 & 3.02300 & -3.18226 \\ H & 0.03301 & 3.98536 & -4.58444 \\ H & -1.42492 & 4.85696 & -5.09974 \\ H & -0.88723 & 6.76709 & -3.68634 \\ H & 0.72954 & 6.31171 & -4.23177 \\ H & 1.30705 & 5.55609 & -2.03174 \\ H & -0.15429 & 6.37511 & -1.49013 \\ H & 0.91075 & 0.99007 & 2.12617 \\ H & -1.52731 & -0.04630 & 0.19421 \text { NewGTO "def2-TZVP(-f)" end } \\ H & -0.83030 & -1.35995 & 2.93904 \\ H & 0.88005 & -1.09690 & 3.25658 \\ H & -1.34431 & -0.25818 & 4.92525 \\ H & 0.32233 & 0.27550 & 5.15372 \\ H & -0.04533 & 2.34718 & 4.03451 \\ H & -1.74014 & 2.06483 & 4.47224 \\ * & & & \end{array}$

\%eprnmr nuclei $=$ all Fe $\{$ rho, fgrad $\}$

end

\section{n Calculated ${ }^{57} \mathrm{Fe}$ Mössbauer Properties - (cyclomet-dicPDI)Fe-H}

$\rho(0)=11582.899659830 \times 10^{-3}-->0.25 \mathrm{~mm} / \mathrm{s}$

$\Delta \mathrm{E}=2.042 \mathrm{~mm} / \mathrm{s}$

- Calculated Thermochemical Properties - (cyclomet-dicPDI)Fe-H (Calculated at 298.15 K)

Final Gibbs free enthalpy $\quad \ldots \quad-3018.8913188246$

- Optimized Coordinates - (cyclomet-dicPDI)Fe-H

$\begin{array}{llrl}\text { C } & 3.74377604815751 & -1.47230575417018 & 0.94806701787382 \\ \text { C } & 2.35043222140790 & -1.40490606885478 & 0.81197071230111 \\ \text { N } & 1.71568817791453 & -0.18953896160972 & 0.85517595708654 \\ \text { C } & 2.43256882810101 & 0.97116367016620 & 0.79842580040626 \\ \text { C } & 3.82064971438567 & 0.94923337945921 & 0.92151691169791 \\ \text { C } & 4.48226792314386 & -0.28878275582217 & 1.02878507993797 \\ \text { C } & 1.45706412282993 & -2.48996198291650 & 0.47567064461781 \\ \text { C } & 1.59529491986909 & 2.14546309555233 & 0.55370039094985 \\ \text { C } & 2.13532059105297 & 3.53638640982285 & 0.78109840030673 \\ \text { N } & 0.38132408509170 & 1.86241920119257 & 0.15830099310002 \\ \text { C } & 1.94594601104748 & -3.91331929478453 & 0.42093788788199 \\ \text { N } & 0.22806492287672 & -2.10560660719470 & 0.15674618079249 \\ \text { C } & -0.67760933614006 & -3.04269181005856 & -0.44461777034693 \\ \text { C } & -0.60095176224387 & 2.88764358500513 & 0.01647415190933 \\ \text { C } & -0.60673815188717 & -3.23184152452534 & -1.84866651085372 \\ \text { C } & -1.46123756471035 & -4.17773552118165 & -2.43518595563180\end{array}$




\begin{tabular}{|c|c|c|c|}
\hline & -2.37933005809703 & -4.89965179957970 & -1.67430770497274 \\
\hline & -2.46599075770668 & -4.66635419484236 & -0.30341846735765 \\
\hline & -1.62711808681271 & -3.74153919454881 & 0.3384323032602 \\
\hline & -1.59793085145504 & 3.00265142299550 & \\
\hline & -2.53804130128502 & 03896244058982 & \\
\hline & -2.53612986333985 & 4.89601928448216 & -0.21155115804714 \\
\hline & -1.59398320587671 & 4.71459412771921 & -1.2228530062087 \\
\hline & -0.61420576242879 & 3.71536312297087 & -1.1349094160259 \\
\hline & 0.37735658784549 & -2.45709535674131 & -2.71240079336360 \\
\hline & -1.74529783800947 & -3.53382376913839 & \\
\hline & & 3.457 & \\
\hline & -1.78847558060038 & 2.00213521844488 & \\
\hline & 1.57422279129089 & -3.312779 & -3.26 \\
\hline & 559287585 & 32429667636 & -4.7939723 \\
\hline & 8437386752 & 85606473809 & -4.97680016 \\
\hline & -0.24113742157290 & -1.78527299626512 & -3.9697235927 \\
\hline & & -3.1 & \\
\hline & -3.2 & -3.6 & \\
\hline & -1.8 & -4.3 & \\
\hline & 9466385 & 0572 & \\
\hline & -0.376 & 2.75791991651 & \\
\hline & 0.670 & 2.851676073443 & -4.6097230310 \\
\hline & 1.2 & $4.2 \varepsilon$ & -4.4 \\
\hline & 0.9 & & -2.9 \\
\hline & -0.7 & 0.90 & \\
\hline & 3057540002 & 0.22 & \\
\hline & & 1.40 & \\
\hline & -2.00 & 2.60 & \\
\hline & -0.2 & -0.1 & \\
\hline & 4.25 & -2.4 & \\
\hline & 4.39 & & \\
\hline & & -0.325 & 1.12 \\
\hline & 89692587645 & 8476 & \\
\hline & 342373 & 3.91 & -0.120 \\
\hline & 2.86 & 3.5 & \\
\hline & 2.5 & -4.0 & -0. \\
\hline & 1.10 & -4.6 & \\
\hline $\mathrm{H}$ & 5887857400 & -4.14 & \\
\hline $\mathrm{H}$ & 6730086448 & & \\
\hline & 83572816839 & 2626209726 & -2.153179 \\
\hline & 28681271244 & -5.22 & \\
\hline & & & \\
\hline & & & -0.2 \\
\hline $\mathrm{H}$ & -1.62916589011317 & & -2.10 \\
\hline $\mathrm{H}$ & 0.80077577269661 & -1.65316218476213 & $-2.0967097979947 \varsigma$ \\
\hline & -1.06628020587097 & -2.71692356941492 & 2.1231666991902 \\
\hline & 1.11877051292169 & 2.77861299474206 & -1.97431531014470 \\
\hline & & & 1.8897 \\
\hline & 1.47695469839642 & -4.37 & 98124773 \\
\hline & 2.51781275615138 & -2.95552 & -2.82 \\
\hline $\mathrm{H}$ & 2.59866441909230 & -3.15519266656384 & -5.22795490032577 \\
\hline & 0.97991424340453 & -3.86585033695959 & $-5.2961298190073 \varsigma$ \\
\hline
\end{tabular}




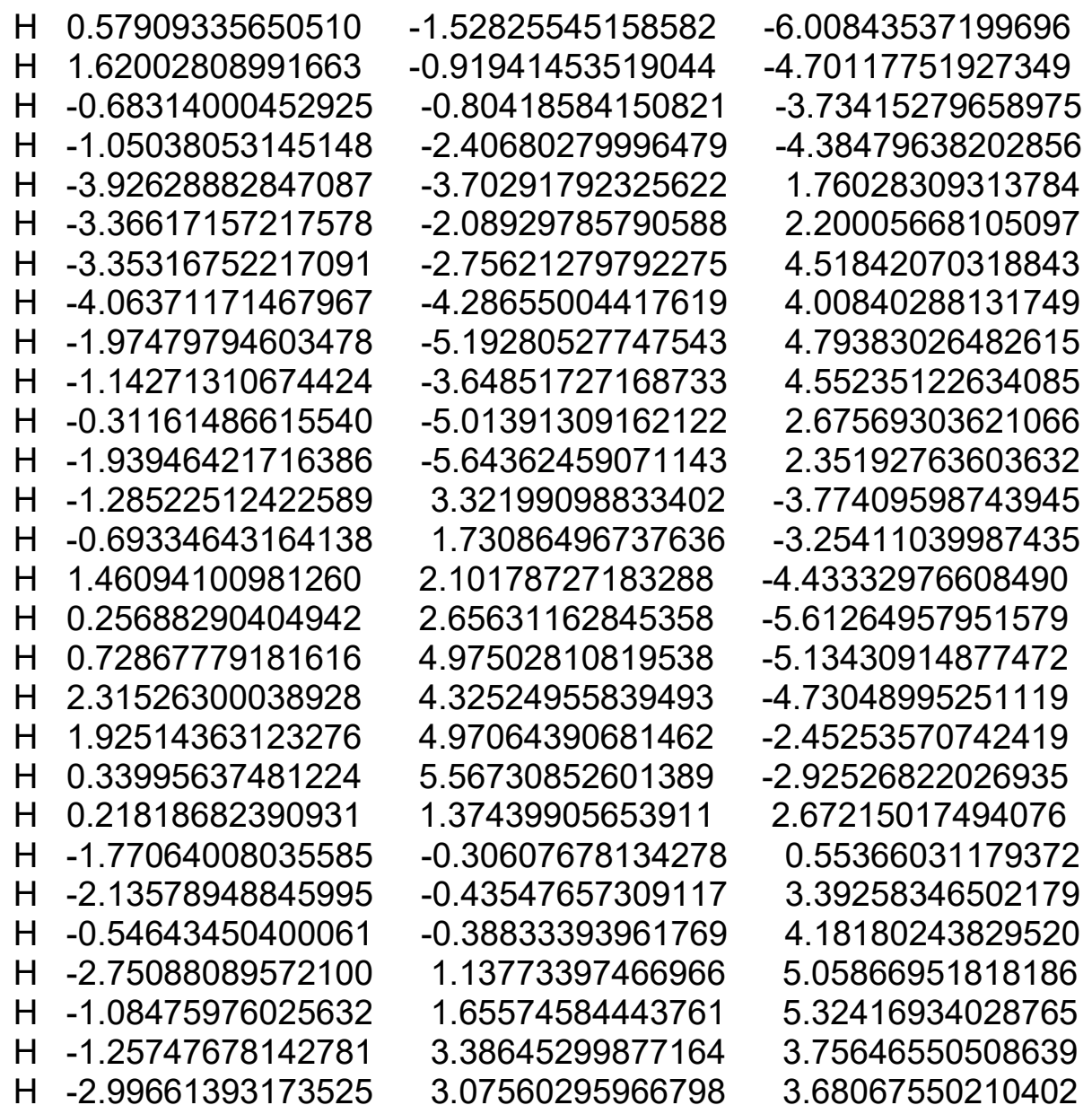

\section{- DFT Input File - (dehydro-dicPDI)Fe( $\left.\mathbf{N}_{2}\right)$ (isomer 1)}

! RKS B3LYP RIJCOSX def2-SVP def2/J Normalprint SlowConv TightSCF Opt Pal8 UCO

\%basis NewGTO 26 "def2-TZVP(-f)" end NewGTO 7 "def2-TZVP(-f)" end NewAuxGTO 26 "def2/J" end NewAuxGTO 7 "def2/J" end end

\%SCF Maxiter 8000

TolE 1e-7

TolErr 1e-6

end

$\begin{array}{lrrr}{ }^{*} \text { xyz } 0 & 1 & & \\ \mathrm{C} & 1.02605 & 3.87384 & 0.27170 \\ \mathrm{C} & 1.08904 & 2.53926 & 0.15992 \\ \mathrm{~N} & 0.00000 & 1.85967 & -0.00000 \\ \mathrm{C} & -1.19219 & 2.38553 & 0.02070 \\ \mathrm{C} & -1.29326 & 3.72476 & 0.06632 \\ \mathrm{C} & -0.17763 & 4.46528 & 0.18593 \\ \mathrm{C} & 2.12620 & 1.69589 & 0.23443\end{array}$




\begin{tabular}{|c|c|c|c|}
\hline C & -2.20415 & 1.48737 & 0.02013 \\
\hline C & -3.62258 & 1.93139 & -0.24370 \\
\hline $\mathrm{N}$ & -1.87875 & 0.21142 & 0.06387 \\
\hline C & 3.51982 & 2.12661 & 0.65417 \\
\hline $\mathrm{N}$ & 1.84191 & 0.42772 & 0.01747 \\
\hline C & 2.84663 & -0.21494 & -0.54604 \\
\hline C & -2.65034 & -0.83672 & 0.14656 \\
\hline C & 3.43345 & -1.26847 & 0.08254 \\
\hline C & 4.48708 & -1.88582 & -0.48346 \\
\hline C & 5.00744 & -1.47773 & -1.64233 \\
\hline C & 4.48130 & -0.39637 & -2.21956 \\
\hline C & 3.42376 & 0.25415 & -1.69845 \\
\hline C & -3.81178 & -1.06701 & 0.79431 \\
\hline C & -4.42441 & -2.24389 & 0.55959 \\
\hline C & -3.91354 & -3.15934 & -0.27648 \\
\hline C & -2.72091 & -2.94496 & -0.84705 \\
\hline C & -2.08786 & -1.78561 & -0.61703 \\
\hline C & 3.09069 & -1.71889 & 1.49245 \\
\hline C & 2.96079 & 1.46687 & -2.53709 \\
\hline C & -4.36376 & -0.15762 & 1.87829 \\
\hline $\mathrm{Fe}$ & 0.00000 & 0.00000 & 0.00000 \\
\hline C & 3.01356 & -3.22924 & 1.77971 \\
\hline C & 2.94450 & -3.28272 & 3.31497 \\
\hline C & 3.78241 & -2.08388 & 3.79872 \\
\hline C & 4.12366 & -1.26487 & 2.54034 \\
\hline C & 3.89454 & 2.69427 & -2.41789 \\
\hline C & 3.93639 & 3.35339 & -3.80264 \\
\hline C & 3.92171 & 2.14371 & -4.73795 \\
\hline C & 2.87959 & 1.23311 & -4.07351 \\
\hline $\mathrm{H}$ & 1.53069 & -0.46915 & -2.73440 \\
\hline C & 0.47688 & -0.62392 & -3.00928 \\
\hline C & 0.29316 & -2.14658 & -3.09396 \\
\hline C & 0.24982 & -2.52314 & -1.61102 \\
\hline C & -5.84102 & 0.25489 & 1.77845 \\
\hline C & -6.12967 & 0.86210 & 3.16143 \\
\hline C & -5.19159 & 0.13253 & 4.14220 \\
\hline C & -4.31825 & -0.79609 & 3.28020 \\
\hline $\mathrm{N}$ & -0.04799 & -1.54877 & 0.99706 \\
\hline$N$ & -0.16921 & -2.56103 & 1.65585 \\
\hline $\mathrm{H}$ & 1.92490 & 4.49139 & 0.41746 \\
\hline $\mathrm{H}$ & -2.27147 & 4.23097 & 0.07078 \\
\hline $\mathrm{H}$ & -0.25423 & 5.56295 & 0.26432 \\
\hline $\mathrm{H}$ & -4.06326 & 2.46651 & 0.62660 \\
\hline $\mathrm{H}$ & -3.63880 & 2.64046 & -1.10226 \\
\hline $\mathrm{H}$ & -4.28946 & 1.10725 & -0.57387 \\
\hline $\mathrm{H}$ & 3.71295 & 3.21075 & 0.51885 \\
\hline $\mathrm{H}$ & 3.60445 & 1.94713 & 1.75017 \\
\hline $\mathrm{H}$ & 4.37103 & 1.59063 & 0.18729 \\
\hline $\mathrm{H}$ & 5.00522 & -2.71909 & 0.01379 \\
\hline $\mathrm{H}$ & 5.88629 & -1.98093 & -2.07864 \\
\hline $\mathrm{H}$ & 4.96450 & -0.04554 & -3.14457 \\
\hline $\mathrm{H}$ & -5.36164 & -2.49990 & 1.07891 \\
\hline
\end{tabular}




\begin{tabular}{|c|c|c|}
\hline-4.43751 & -4.11648 & -0.43862 \\
\hline-2.27479 & -3.73217 & -1.47332 \\
\hline 2.13685 & -1.25543 & 1.81196 \\
\hline 1.93633 & 1.78498 & -2.23059 \\
\hline-3.72879 & 0.75249 & 1.97924 \\
\hline 2.16156 & -3.73247 & 1.27439 \\
\hline 3.93818 & -3.76763 & 1.47265 \\
\hline 3.31212 & -4.25343 & 3.71947 \\
\hline 1.88683 & -3.15843 & 3.64714 \\
\hline 3.19056 & -1.46708 & 4.51599 \\
\hline 4.71048 & -2.41401 & 4.32090 \\
\hline 5.15398 & -1.52460 & 2.20071 \\
\hline 4.09529 & -0.16754 & 2.72546 \\
\hline 3.55162 & 3.43557 & -1.66374 \\
\hline 4.92837 & 2.37123 & -2.15097 \\
\hline 4.82900 & 4.00320 & -3.94892 \\
\hline 3.01766 & 3.96723 & -3.96209 \\
\hline 3.66169 & 2.41274 & -5.78706 \\
\hline 4.92442 & 1.65582 & -4.74192 \\
\hline 2.99749 & 0.17542 & -4.39663 \\
\hline 1.87975 & 1.56788 & -4.44483 \\
\hline 0.28378 & -0.14498 & -3.99564 \\
\hline-0.67124 & -2.40013 & -3.59443 \\
\hline 1.12526 & -2.65121 & -3.63678 \\
\hline 1.25617 & -2.39612 & -1.15367 \\
\hline-0.06411 & -3.57433 & -1.44069 \\
\hline-6.05554 & 0.96442 & 0.95003 \\
\hline-6.49473 & -0.63602 & 1.63535 \\
\hline-7.20288 & 0.76018 & 3.44276 \\
\hline-5.88415 & 1.95135 & 3.14408 \\
\hline-4.55032 & 0.87176 & 4.67863 \\
\hline-5.75770 & -0.44472 & 4.90956 \\
\hline-4.77816 & -1.81172 & 3.26502 \\
\hline-3.28026 & -0.90070 & 3.67140 \\
\hline-0.72087 & -1.48038 & -1.09193 NewGTO "def2-TZVP(-f)" end \\
\hline-0.41385 & -0.02359 & -1.93284 NewGTO "def2-TZVP(-f)" end \\
\hline-1.21310 & 0.66431 & -2.19885 \\
\hline
\end{tabular}

\%eprnmr nuclei $=$ all Fe $\{$ rho, fgrad $\}$

end

- Calculated ${ }^{57}$ Fe Mössbauer Properties: (dehydro-dicPDI)Fe( $\left.\mathbf{N}_{2}\right)$ (isomer 1)

$\rho(0)=11582.492243668 \times 10^{-3}-->0.375 \mathrm{~mm} / \mathrm{s}$

$\Delta \mathrm{E}=0.944 \mathrm{~mm} / \mathrm{s}$

- Optimized Coordinates - (dehydro-dicPDI)Fe( $\left.\mathbf{N}_{2}\right)$ (isomer 1$)$
C 1.04518486487010
$4.06560708227877 \quad 0.83362932927375$
C 1.06625379162556
$2.75812444989134 \quad 0.34112052611639$ 


\begin{tabular}{|c|c|c|c|}
\hline & -0.07707834243491 & .97909167080137 & \\
\hline & -1.25666504121336 & 2.49731311952099 & 0.88920330359189 \\
\hline & -1.29488089763338 & .79991623831682 & \\
\hline & .13730497458521 & 59054450039817 & 388 \\
\hline & 2.19711427220145 & 2.00644615240399 & 1877411997641 \\
\hline & -2.33976484144293 & 1.56005453745827 & 0.7597663985669 \\
\hline & -3.76664035840390 & 1.95498157262114 & 1.0208050194839 \\
\hline & -1.94025087663058 & 0.39718638782125 & \\
\hline & 3.59200091085528 & & \\
\hline & 32135120 & & \\
\hline & 2.94431065546223 & -0.22218272118219 & -0.4 \\
\hline & -2.78367290837543 & 366347739 & \\
\hline & 3.29324516147635 & 37136871 & 0.750 \\
\hline & 4.24230406318830 & -1.84118863206681 & \\
\hline & 4.84556692955018 & -2.28712444411111 & -0.414231 \\
\hline & 4.5 & -1.6 & \\
\hline & 3.58 & -0.6 & \\
\hline & -3.76 & 088 & \\
\hline & -4.47 & 650 & \\
\hline ; & 16975 & 791052196 & \\
\hline & 34256 & 05889474 & -2.23256836617 \\
\hline & -2.4 & 043 & \\
\hline & 2.70 & & \\
\hline & 3.36 & 0.0 & -3.0 \\
\hline & & 1277 & \\
\hline & -0.10480179 & 6510 & -0.43 \\
\hline & 1.995274592 & & \\
\hline & 2.44 & -1.23 & \\
\hline & & & \\
\hline C & 3.73 & & \\
\hline & 4.456115014 & & -3.2 \\
\hline & 4.879353948 & & \\
\hline & 4.737 & $-0.4 \varepsilon$ & \\
\hline & 3.40 & -0.8 & \\
\hline & $0.7 \varepsilon$ & & \\
\hline & -0.2 & & \\
\hline 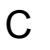 & -0.223 & & \\
\hline C & -0.56236144822811 & 39198502 & -3. \\
\hline C & -5.40020831051170 & -0.9 & \\
\hline & -5.39859642443207 & -1.3 & \\
\hline & -4.0 & -2.0 & \\
\hline & & & \\
\hline N & & & -0.29 \\
\hline N & 0.35267949395746 & -2.58202113518454 & 08157144826 \\
\hline $\mathrm{H}$ & 1.95503574949335 & 4.66920378607681 & 0.80750686262801 \\
\hline & -2.22308170034861 & 4.1969 & 1.8108896310292 \\
\hline & -0.15 & 5.6 & 1.772 \\
\hline & -4.02110183144128 & & \\
\hline $\mathrm{H}$ & -3.94797912552295 & 2.98239093389874 & 4846434910 \\
\hline $\mathrm{H}$ & -4.46611297408867 & 1.29059961511788 & 0.4974096156021 \\
\hline $\mathrm{H}$ & 3.70829406752592 & 3.42057488500808 & -0.73589822284118 \\
\hline & 3.82057954714801 & 2.95291005674927 & 0.967349 \\
\hline
\end{tabular}



H 4.34777646287288
1.82015807820789
$-0.29858237590500$
H 4.51355217020320
$-2.30609027883675$
1.71152728905523
H 5.57510761285602
$-3.10103405697238$
$-0.38515058834481$
H 5.02376530236831
$-2.00908488513965$
$-2.53476702158771$
H $\quad-5.23769316269526$
$-2.90866306382469$
0.30184569257449
H -4.76274225421971
$-3.42834832101520$
H -2.92605449586414
$-2.26417610345170$
$-2.06648475055291$
H 1.94654978544244
0.41782352268378
$-3.25841574589993$
H 2.38215079307698
0.58476868221538
1.86706165264319
H $\quad-3.32816580891300$
$-0.34697745377910$
$-2.95647471141451$
H 0.90294578134024
$-1.35773675387305$
2.31068661024603
H 2.24849693484775
$-2.46744638911442$
H 3.23142429884681
$-1.96213784929454$
H 1.63074921951742
$-1.36914934100126$
H 2.24990810116328
0.93935277297404
H 3.73953125598391
0.35573115133958
H 4.66359784345803
$-0.31748982186408$
H 4.01935425429176
1.30461586947744
H 4.09022177270569
2.20172881107689
1.00766689958604
2.82572398086076
2.57959598515360
4.66496473114037
5.12296679347342
4.49311829833465
5.26251075003945
3.05764516176220
2.76321296862058
H 5.32518943873149
1.41227829873323
$-3.05448500704202$
$-2.62768438144300$
H 5.89016155437846
H 4.18448648638655
1.56988242560473
$-4.95595700622805$
$-5.41406427698803$
$-0.76382995308575$
$-6.06007638157948$
H 5.57563005602837
$-1.02075309668564$
$-4.51878015122811$
$-1.87514165434130$
$-4.06772760204328$
H 2.58639738869969
$-0.51375427067020$
2.48723794399999
0.52762465606009
H -1.01619695155520
0.27728822273670
H 0.71960497286634
H 0.34021182080512
H -1.17201375861712
$-1.38869292143685$
$-1.56894358564963$
0.08919399912160
H $\quad-5.73026261436712$
H -6.09400816880431
$-1.58506062312670$
$-4.96308794548880$
$-3.81178400073892$
$-5.06955995184499$
$-4.86915178585589$
$-3.13016322294171$
$-4.00223415500489$
2.30231436405473
1.88812082014561
$-2.05148905913679$
4.17188448596973
$-0.50400991706648$
4.62560221733244
$-1.37855997929556$
4.68395957402578
$-2.95604523122680$
4.85247478456090
$-3.31727932281393$
$-2.60677672234313$
$-0.25496818805973$
2.40184917798193
2.74745750241237
H -2.43728247582598
C -1.30873362343892
1.11999987391795
$-2.24759306447422$
C -1.05935154475449
1.82060949766857
$-2.17731217250751$
$-1.72387847180139$

\section{- DFT Input File - (dehydro-dicPDI)Fe( $\left.\mathbf{N}_{2}\right)$ (isomer 2)}

! RKS B3LYP RIJCOSX def2-SVP def2/J Normalprint SlowConv TightSCF Opt Pal8 UCO

\%basis NewGTO 26 "def2-TZVP(-f)" end NewGTO 7 "def2-TZVP(-f)" end

NewAuxGTO 26 "def2/J" end

NewAuxGTO 7 "def2/J" end end 


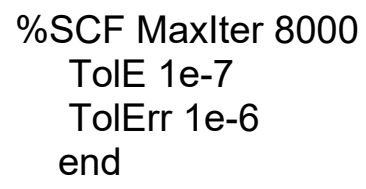




\begin{tabular}{|c|c|c|c|}
\hline $\mathrm{N}$ & -0.29319 & -2.72882 & 1.26488 \\
\hline $\mathrm{H}$ & 1.97400 & 4.48274 & 0.10269 \\
\hline $\mathrm{H}$ & -2.24825 & 4.24703 & 0.12287 \\
\hline $\mathrm{H}$ & -0.21323 & 5.57112 & 0.10719 \\
\hline $\mathrm{H}$ & -4.38571 & 1.11835 & -0.05499 \\
\hline $\mathrm{H}$ & -3.90122 & 2.44854 & 1.04651 \\
\hline $\mathrm{H}$ & -3.82377 & 2.64314 & -0.74842 \\
\hline $\mathrm{H}$ & 3.83368 & 2.91423 & -0.58913 \\
\hline $\mathrm{H}$ & 3.60804 & 2.80402 & 1.18252 \\
\hline $\mathrm{H}$ & 4.35264 & 1.45409 & 0.32517 \\
\hline $\mathrm{H}$ & 4.82395 & -2.78322 & 0.92841 \\
\hline $\mathrm{H}$ & 5.86014 & -2.55075 & -1.21541 \\
\hline $\mathrm{H}$ & 5.05596 & -0.90174 & -2.77026 \\
\hline $\mathrm{H}$ & -5.13112 & -2.49228 & 1.51861 \\
\hline $\mathrm{H}$ & -5.34157 & -3.35475 & -0.72171 \\
\hline $\mathrm{H}$ & -3.92224 & -2.53796 & -2.49330 \\
\hline $\mathrm{H}$ & 1.74353 & -1.17853 & 1.98659 \\
\hline $\mathrm{H}$ & 2.17891 & 1.36657 & -2.31104 \\
\hline $\mathrm{H}$ & -2.75735 & 0.26127 & 2.47056 \\
\hline $\mathrm{H}$ & 2.27914 & -3.55978 & 2.29021 \\
\hline $\mathrm{H}$ & 3.96014 & -3.14091 & 2.80878 \\
\hline $\mathrm{H}$ & 2.83507 & -3.09441 & 4.93099 \\
\hline $\mathrm{H}$ & 1.35830 & -2.35561 & 4.20864 \\
\hline $\mathrm{H}$ & 2.23103 & -0.28046 & 4.76393 \\
\hline $\mathrm{H}$ & 3.87213 & -0.94502 & 5.10254 \\
\hline $\mathrm{H}$ & 4.61754 & -0.60044 & 2.90523 \\
\hline $\mathrm{H}$ & 3.27975 & 0.60004 & 2.80092 \\
\hline $\mathrm{H}$ & 3.81591 & 2.79322 & -2.91426 \\
\hline $\mathrm{H}$ & 5.11025 & 1.64680 & -2.43465 \\
\hline $\mathrm{H}$ & 5.19824 & 0.69469 & -4.65973 \\
\hline $\mathrm{H}$ & 4.80547 & 2.41211 & -5.05850 \\
\hline $\mathrm{H}$ & 2.37693 & 1.88326 & -5.12184 \\
\hline $\mathrm{H}$ & 3.14865 & 0.52560 & -6.04117 \\
\hline $\mathrm{H}$ & 3.13983 & -0.90998 & -4.07193 \\
\hline $\mathrm{H}$ & 1.53207 & -0.12267 & -3.99692 \\
\hline $\mathrm{H}$ & 0.37178 & -3.20783 & -2.34280 \\
\hline $\mathrm{H}$ & -0.92532 & -2.92898 & -1.15044 \\
\hline $\mathrm{H}$ & -1.96921 & -2.68736 & -3.41242 \\
\hline $\mathrm{H}$ & -0.67305 & -1.56777 & -3.92728 \\
\hline $\mathrm{H}$ & -5.14098 & 0.88598 & 2.53830 \\
\hline $\mathrm{H}$ & -5.61027 & -0.80528 & 2.94083 \\
\hline $\mathrm{H}$ & -5.35655 & 0.03030 & 5.16141 \\
\hline $\mathrm{H}$ & -4.05053 & 1.17027 & 4.67095 \\
\hline $\mathrm{H}$ & -2.45611 & -0.39182 & 5.22923 \\
\hline $\mathrm{H}$ & -3.70540 & -1.63415 & 5.60530 \\
\hline $\mathrm{H}$ & -3.65151 & -2.53345 & 3.42309 \\
\hline $\mathrm{H}$ & -1.97151 & -1.88848 & 3.42266 \\
\hline $\mathrm{H}$ & -2.49395 & -0.08482 & -3.02199 \\
\hline C & -1.90039 & -0.71394 & -2.31714 \\
\hline C & -0.65791 & 0.07077 & -1.85917 NewGTO "def2-TZVP(-f)" end \\
\hline $\mathrm{H}$ & -0.28158 & 0.94731 & -2.38482 \\
\hline
\end{tabular}


\%eprnmr nuclei $=$ all Fe $\{$ rho, fgrad $\}$

end

n Calculated ${ }^{57} \mathrm{Fe}$ Mössbauer Properties: (dehydro-dicPDI)Fe( $\left.\mathbf{N}_{2}\right)$ (isomer 2)

$\rho(0)=11582.492139974 \times 10^{-3}-->0.374 \mathrm{~mm} / \mathrm{s}$

$\Delta \mathrm{E}=-0.937 \mathrm{~mm} / \mathrm{s}$

\section{- Optimized Coordinates - (dehydro-dicPDI)Fe( $\left.\mathbf{N}_{2}\right)$ (isomer 2)}

\begin{tabular}{|c|c|c|c|}
\hline & & & \\
\hline & 1.10581782385237 & 2.71086587030417 & \\
\hline & -0.06910049632065 & .98649948314252 & 21454687803322 \\
\hline & & 51959276198532 & \\
\hline & & & \\
\hline & .06327278297129 & 4.60115322205017 & 1.16194235216376 \\
\hline & 2.21832863330691 & 1.89099339936489 & -0.17600443025363 \\
\hline & -2.29943141240910 & 1.56905799557423 & 0.72172001180047 \\
\hline & -3.70685118647377 & 1.93257947590343 & \\
\hline & & 0.38577427729269 & \\
\hline & & & \\
\hline & & & \\
\hline & 2.89 & -0.3 & -0.2 \\
\hline & -2.86 & & 8510 \\
\hline & 3.159 & & \\
\hline & 4.137 & & 1.20 \\
\hline & 4.8 & -2.3 & \\
\hline & & & \\
\hline & & & \\
\hline & & 069 & \\
\hline & -4.35 & -2.4 & \\
\hline & & & -0.82 \\
\hline & -4.1 & -1.7 & 2477 \\
\hline & & & \\
\hline & & & \\
\hline & & & 26 \\
\hline & & -0.37 & -2.80 \\
\hline & -3.01 & -1.35 & 2.37 \\
\hline & -0.1 & 0.3 & -0.5 \\
\hline & & -1.25 & \\
\hline & 1.7 & -0.4 & \\
\hline & & & \\
\hline & 3.3624707 & 0.64 & 2822 \\
\hline & 4.714 & 0.36 & -3.36 \\
\hline & 4.756 & & -4.89 \\
\hline & & $-0.6 s$ & \\
\hline & & -1.44 & -3.8 \\
\hline & & & \\
\hline & -0.37120392426598 & -0.59385589435636 & \\
\hline & -1.90590683851703 & -0.79226659739347 & -3.720499239019 \\
\hline
\end{tabular}




\begin{tabular}{|c|c|c|c|}
\hline & -4.14868731775893 & -1.03156676092712 & \\
\hline & -3.52227594392150 & -1.34259000441717 & 0876 \\
\hline & 99674834126 & 9382 & \\
\hline & -2.42536521988051 & -2.70400651234485 & 14728 \\
\hline & -0.01211402448340 & -1.53242363950704 & -0.6573299987726 \\
\hline & .06328274638552 & -2.63744601505761 & -0.6683437278892 \\
\hline & 2.03532779421922 & 4.61668224897287 & 0.6203649273470 \\
\hline & -2.14778667236421 & 4.25169701376894 & 1.65652163769844 \\
\hline & 612242 & 0851 & \\
\hline & 76858 & 2619 & 0. \\
\hline & +575592 & 2.11614473188573 & 2.1976648738818 \\
\hline & 2478483 & 2.85622010920795 & 0.6057423611992 \\
\hline & 3.796 & 3.00595516208489 & -1.141150462824 \\
\hline & 3.832 & 3.10327858339688 & 0.627873022 \\
\hline & & & \\
\hline & & & \\
\hline & 5.59 & -3.12 & \\
\hline & 56526 & -2.30585 & \\
\hline & 456003 & 34439781 & \\
\hline & 711980 & 2848635 & -1.12016696 \\
\hline & -4.3 & -1.9 & \\
\hline & & & \\
\hline & 2.61 & & \\
\hline & 628036 & 19734 & \\
\hline & & & \\
\hline & 2.57 & -2.10 & 3.4 \\
\hline & 2.20 & -1.0 & \\
\hline & & -0. & \\
\hline & 1.7 & & \\
\hline & 2642 & & \\
\hline & 4.281 & & \\
\hline & 3.678 & 282456 & 2.61 \\
\hline & 4.658 & 1.4 & -3.1 \\
\hline & & 0.0 & \\
\hline & 09 & -0.5 & \\
\hline & 4.871 & & \\
\hline & 2.63141950973433 & 0.01 & \\
\hline & 3.52309416981766 & -1.358260467178 & -6.07 \\
\hline & 3.87733823 & -2.2863880177 & -3.78 \\
\hline & & -1.86 & \\
\hline & & & \\
\hline & 0.13911312402988 & -1.50171812291084 & -3.28 \\
\hline & -2.19214417608952 & -1.85240289230879 & 245456 \\
\hline & -2.28238132484893 & -0.39187534657615 & -4.6746040771828 \\
\hline & -4.50138900747836 & 0.00675831765601 & 3.26 \\
\hline & -5.01 & -1.6 & \\
\hline & -4.25 & -1.3 & \\
\hline & -2.79676523176433 & -0.55252754621302 & 4.9894914000093 \\
\hline & -1.90965390228953 & -2.80248141293629 & 5.1443461947756 \\
\hline & -3.46718817359928 & -3.52145056501789 & 4.7340062048108 \\
\hline & & & \\
\hline & $-1.33967 t$ & & \\
\hline
\end{tabular}

S80 


$\begin{array}{llll}H & -3.34116921044072 & 0.66916061809533 & -2.95591841775087 \\ \mathrm{C} & -2.53178142455774 & 0.03642709703495 & -2.54710727506733 \\ \mathrm{C} & -1.38900174713873 & 0.95886049945367 & -2.14071268026356 \\ \mathrm{H} & -1.60620300243558 & 1.96656672319159 & -1.81245020319126\end{array}$

- DFT Input File -1,7-octadiene

\begin{tabular}{|c|c|c|c|}
\hline \\
\hline \multicolumn{4}{|c|}{$\begin{array}{l}\text { ! RKS B3LYP RIJCOSX def2-SVP def2/J N } \\
\text { NumFreq Grid5 FinalGrid6 GridX4 Pal8 UC }\end{array}$} \\
\hline \multicolumn{4}{|c|}{$\begin{array}{l}\text { \%SCF Maxlter } 5000 \\
\text { Convergence VeryTight } \\
\text { end }\end{array}$} \\
\hline \multicolumn{4}{|c|}{$\begin{array}{l}\text { \%freq increment } 0.01 \\
\text { restart true } \\
\text { centraldiff true } \\
\text { end }\end{array}$} \\
\hline \multicolumn{4}{|c|}{ * xyz 01} \\
\hline C & -7.60874 & 0.82818 & 0.00433 \\
\hline C & -6.50140 & 1.58142 & -0.00616 \\
\hline C & -5.11703 & 0.99771 & -0.00602 \\
\hline C & -4.03532 & 2.08187 & 0.00751 \\
\hline $\mathrm{H}$ & -4.98964 & 0.37291 & -0.8984 \\
\hline $\mathrm{H}$ & -4.99554 & 0.35569 & 0.8749 \\
\hline C & -2.63424 & 1.46574 & 0.0102 \\
\hline $\mathrm{H}$ & -4.15785 & 2.71784 & 0.89340 \\
\hline $\mathrm{H}$ & -4.14897 & 2.72932 & -0.87126 \\
\hline C & -1.54950 & 2.54671 & 0.01552 \\
\hline $\mathrm{H}$ & -2.51454 & 0.82515 & -0.87268 \\
\hline $\mathrm{H}$ & -2.51965 & 0.82222 & 0.8918 \\
\hline C & -0.16805 & 1.95557 & 0.0102 \\
\hline $\mathrm{H}$ & -1.67020 & 3.17496 & 0.9063 \\
\hline $\mathrm{H}$ & -1.67236 & 3.18596 & -0.8672 \\
\hline C & 0.94422 & 2.70133 & 0.0002 \\
\hline $\mathrm{H}$ & -8.59323 & 1.28574 & 0.00342 \\
\hline $\mathrm{H}$ & -7.55837 & -0.25635 & 0.0129 \\
\hline $\mathrm{H}$ & -6.60554 & 2.66437 & -0.01587 \\
\hline $\mathrm{H}$ & -0.07041 & 0.87190 & 0.01423 \\
\hline $\mathrm{H}$ & 0.90155 & 3.78616 & -0.00371 \\
\hline $\mathrm{H}$ & 1.92542 & 2.23664 & -0.00376 \\
\hline
\end{tabular}

end

- Calculated Thermochemical Properties - 1,7-octadiene (Calculated at 298.15 K)

Total enthalpy $\quad \ldots \quad-312.58312929 \mathrm{Eh}$

Total entropy correction $\quad \ldots \quad-0.04472966 \mathrm{Eh} \quad-28.07 \mathrm{kcal} / \mathrm{mol}$ 
Final Gibbs free enthalpy $\ldots \quad-312.62785895 \mathrm{Eh}$

- Optimized Coordinates -1,7-octadiene
C -7.51093500305775
0.96358423297619
0.32286638441184
C -6.50349062806947
1.47804837200098
0.96907605932426
$-0.39056593619166$
C -5.08840348715993
2.02152712141279
$-0.40577069749682$
C -4.05634778570358
0.64310215868239
0.03517572767222
H -4.83530084324166
0.07350590254524
H $\quad-5.00680529334766$
1.51837332234612
C -2.61094665699910
H -4.29195233801094
2.35076003390704
2.92013072108380
H -4.15781023941488
2.57169068861548
C -1.57849508717003
H -2.37601683200584
1.18749280008168
0.62078224320011
H -2.50896664799913
2.06181698558196
C -0.16373309620146
2.90112194737985
$-1.43338933315372$
0.23513757560250
$-0.02369382555814$
1.06373130324971
$-0.60140859237649$
0.41444267310171
$-1.05187591384560$
0.61423349191041
H -1.83152258209989
3.46517425886315
0.40051933533374
H -1.65960094956771
2.57283334669223
1.44096822622614
C 0.84343526041996
1.39150934861072
$-0.22943289475005$
H $\quad-8.51752477144023$
H $\quad-7.36736212331062$
0.09140453799013
$-0.31578903749016$
0.28495352110822
2.35548549832149
0.97138497161623
H $\quad-6.69956972425965$
1.18678669129557
$-1.02345898743233$
H 0.03227723148719
3.44243372313963
1.03675511190256
H 0.69990481267076
2.14440000594919
$-0.96776630579448$
$-0.27690679804581$

\section{- DFT Input File - cis-bicyclo[4.2.0]octane}

! RKS B3LYP RIJCOSX def2-SVP def2/J Normalprint SlowConv TightSCF TightOpt CPCM(toluene) NumFreq Grid5 FinalGrid6 GridX4 Pal8 UCO
\%SCF Maxlter 5000
Convergence VeryTight
end
\%freq increment 0.01
restart true
centraldiff true
end

$\begin{array}{lrrr}{ }^{*} \text { xyz } 01 & & \\ \text { C } & -7.43775 & 2.85127 & -0.22419 \\ \text { C } & -6.35880 & 2.64870 & 0.86221 \\ \text { C } & -7.95497 & 1.43263 & 0.08028 \\ \text { H } & -7.05030 & 2.98024 & -1.23907 \\ \text { H } & -8.15294 & 3.65385 & -0.01417 \\ \text { C } & -6.59200 & 1.11303 & 0.74136 \\ \text { H } & -8.21992 & 0.84319 & -0.80353 \\ \text { H } & -8.79713 & 1.40854 & 0.78001 \\ \text { C } & -4.95543 & 3.19459 & 0.61663 \\ \text { H } & -6.71888 & 3.03031 & 1.83017\end{array}$




$\begin{array}{lrrr}\mathrm{C} & -5.61991 & 0.32859 & -0.13331 \\ \mathrm{H} & -6.68702 & 0.63097 & 1.72400 \\ \mathrm{C} & -4.20351 & 0.83517 & 0.10237 \\ \mathrm{H} & -5.86605 & 0.43737 & -1.19769 \\ \mathrm{H} & -5.68498 & -0.73987 & 0.09899 \\ \mathrm{C} & -4.11658 & 2.30649 & -0.29892 \\ \mathrm{H} & -3.49898 & 0.24602 & -0.49376 \\ \mathrm{H} & -3.92314 & 0.71428 & 1.15695 \\ \mathrm{H} & -4.44636 & 2.42248 & -1.33921 \\ \mathrm{H} & -3.07189 & 2.63561 & -0.26046 \\ \mathrm{H} & -4.43551 & 3.28149 & 1.57879 \\ \mathrm{H} & -5.01037 & 4.20744 & 0.20124 \\ * & & & \end{array}$

end

- Calculated Thermochemical Properties - cis-bicyclo[4.2.0]octane (Calculated at 298.15 K)

Total enthalpy $\quad \ldots \quad-312.60276708$ Eh

Total entropy correction $\quad \ldots \quad-0.03779046$ Eh $\quad-23.71 \mathrm{kcal} / \mathrm{mol}$

Final Gibbs free enthalpy $\quad \ldots \quad-312.64055755$ Eh

- Optimized Coordinates - cis-bicyclo[4.2.0]octane

$\begin{array}{ll}\text { C } & -7.44160611661564 \\ \text { C } & -6.37178379970597 \\ \text { C } & -7.98823505740164 \\ \text { H } & -6.99953145202263 \\ H & -8.12566885921689 \\ \text { C } & -6.61923801153375 \\ H & -8.31179199333642 \\ H & -8.80158960188165 \\ \text { C } & -4.94126542380244 \\ H & -6.79864420285148 \\ \text { C } & -5.63360664211578 \\ H & -6.65848722452080 \\ \text { C } & -4.18678837828399 \\ H & -5.92009876657642 \\ H & -5.70998766524272 \\ \text { C } & -4.08135900173810 \\ H & -3.51730872681017 \\ H & -3.84278864314724 \\ H & -4.40336237254532 \\ H & -3.03205565114436 \\ H & -4.45756001819677 \\ H & -4.95966239130979\end{array}$
2.81123325220649

2.66195970121871

1.41652441227909

2.80175855501369

3.67276173030506

1.11808887653732

0.73460960877208

1.50036939003023

3.18332591513265

3.06628387800848

0.36299220553069

0.62983159902617

0.84723341030618

0.50518687045821

$-0.72147979023131$

2.34218340693994

0.26236869502717

0.67136414512253

2.50128222221919

2.67941424493805

3.17428912232190

4.24080854883747
$-0.28899076473511$

0.82825803353023

0.11758502283753

$-1.29867436810189$

$-0.22047647851903$

0.79249305400108

$-0.68616339688014$

0.85612397311526

0.66998204260220

1.76201676661873

$-0.11905307991414$

1.78146469966896

0.04401669317250

$-1.17734658161633$

0.07153087476176

$-0.28507869083045$

$-0.60970059668946$

1.08142706846383

$-1.33127863890777$

$-0.23283628234853$

1.66428396350439

0.34910668626637

\section{- DFT Input File - trans-bicyclo[4.2.0]octane}

! RKS B3LYP RIJCOSX def2-SVP def2/J Normalprint SlowConv TightSCF TightOpt NumFreq Grid5 FinalGrid6 GridX4 Pal8 UCO 


\begin{tabular}{|c|c|c|c|}
\hline \multicolumn{4}{|c|}{$\begin{array}{l}\text { \%SCF Maxlter } 5000 \\
\text { Convergence VeryTight } \\
\text { end }\end{array}$} \\
\hline \multicolumn{4}{|c|}{$\begin{array}{l}\text { \%freq increment } 0.01 \\
\text { restart true } \\
\text { centraldiff true } \\
\text { end }\end{array}$} \\
\hline \multicolumn{4}{|c|}{ * xyz 01} \\
\hline C & -7.68404 & 2.96363 & -0.25862 \\
\hline C & -6.22441 & 2.55686 & -0.58318 \\
\hline C & -7.97176 & 1.42851 & -0.20045 \\
\hline $\mathrm{H}$ & -8.21198 & 3.51050 & -1.04353 \\
\hline $\mathrm{H}$ & -7.81085 & 3.48616 & 0.69622 \\
\hline C & -6.50205 & 1.29166 & 0.27154 \\
\hline $\mathrm{H}$ & -8.18347 & 0.96892 & -1.17251 \\
\hline $\mathrm{H}$ & -8.73422 & 1.12085 & 0.51914 \\
\hline C & -4.94609 & 3.18724 & -0.06841 \\
\hline $\mathrm{H}$ & -6.12566 & 2.35538 & -1.66330 \\
\hline C & -5.49228 & 0.23496 & -0.12897 \\
\hline $\mathrm{H}$ & -6.44235 & 1.46384 & 1.35955 \\
\hline C & -4.09677 & 0.77291 & 0.24617 \\
\hline $\mathrm{H}$ & -5.53858 & 0.02511 & -1.20399 \\
\hline $\mathrm{H}$ & -5.68579 & -0.70456 & 0.39903 \\
\hline C & -3.80810 & 2.17877 & -0.32396 \\
\hline $\mathrm{H}$ & -3.32861 & 0.07251 & -0.10039 \\
\hline $\mathrm{H}$ & -4.01593 & 0.82006 & 1.33966 \\
\hline $\mathrm{H}$ & -3.65029 & 2.08734 & -1.40622 \\
\hline $\mathrm{H}$ & -2.87482 & 2.56268 & 0.10298 \\
\hline $\mathrm{H}$ & -5.01491 & 3.41439 & 1.00192 \\
\hline $\mathrm{H}$ & -4.73946 & 4.12625 & -0.59222 \\
\hline
\end{tabular}

$\%$ plots format cube

$\operatorname{dim} 1100 \operatorname{dim} 2100 \operatorname{dim} 3100$

SpinDens("dicPDIFemetallacis2.cube");

end

n Calculated Thermochemical Properties - trans-bicyclo[4.2.0]octane (Calculated at 298.15 K)

\begin{tabular}{llll} 
Total enthalpy & $\ldots$ & $-312.59421761 \mathrm{Eh}$ & \\
Total entropy correction & $\ldots$ & $-0.03751361 \mathrm{Eh}$ & $-23.54 \mathrm{kcal} / \mathrm{mol}$ \\
\hline Final Gibbs free enthalpy & & $\ldots$ & $-312.63173122 \mathrm{Eh}$
\end{tabular}

- Optimized Coordinates - trans-bicyclo[4.2.0]octane

C $\quad-7.70600662382361 \quad 2.96832436818904 \quad-0.26719108530735$ 

C -6.22390693061666
C -7.99485125320072
H $\quad-8.22731189791782$
H $\quad-7.85291071733274$
C -6.50575965561147
H -8.20720052178153
H $\quad-8.76591248651101$
C -4.92086855445839
H -6.13581915432801
C -5.48476939424585
H -6.44591834116116
C -4.10179041417488
H $\quad-5.51811834928662$
H $\quad-5.65558977031066$
C -3.81211428832306
H -3.29717780903680
H -4.04610192803755
H -3.67832853914613
H -2.84594170492205
H -4.96649764463646
H $\quad-4.68952402113681$

2.58656198589680

1.43166129996996

3.56227400992567

3.44689317740802

1.26230003222286

1.01963469440629

1.07745404736884

3.23044795846959

2.35981052256290

0.18596551242539

1.46212251464996

0.77431529310226

$-0.05467426673546$

$-0.75791278687390$

2.17890738861056

0.08454659384926

0.83338142235647

2.08520449307440

2.53889470754962

3.48398718466125

4.16386984691022
$-0.51687278861659$

$-0.19296847972871$

$-1.03437298056612$

0.71532661506995

0.20479788531058

$-1.19364477795032$

0.50906974946551

$-0.07131163635611$

$-1.59748654048507$

$-0.12613921245516$

1.29255719839852

0.25410598896320

$-1.20473811122956$

0.42085789486354

$-0.33170358805738$

$-0.05328579112606$

1.35714508285818

$-1.42556295614644$

0.06182942936731

1.00385186283233

$-0.61380375910425$

\section{- DFT Input File - (dicPDI)Fe(trans-bimetallacyclo[4.3.0]nonane)}

! UKS B3LYP RIJCOSX def2-SVP def2/J Normalprint SlowConv TightSCF TightOpt NumFreq Grid5 FinalGrid6 GridX4 Pal8 UCO

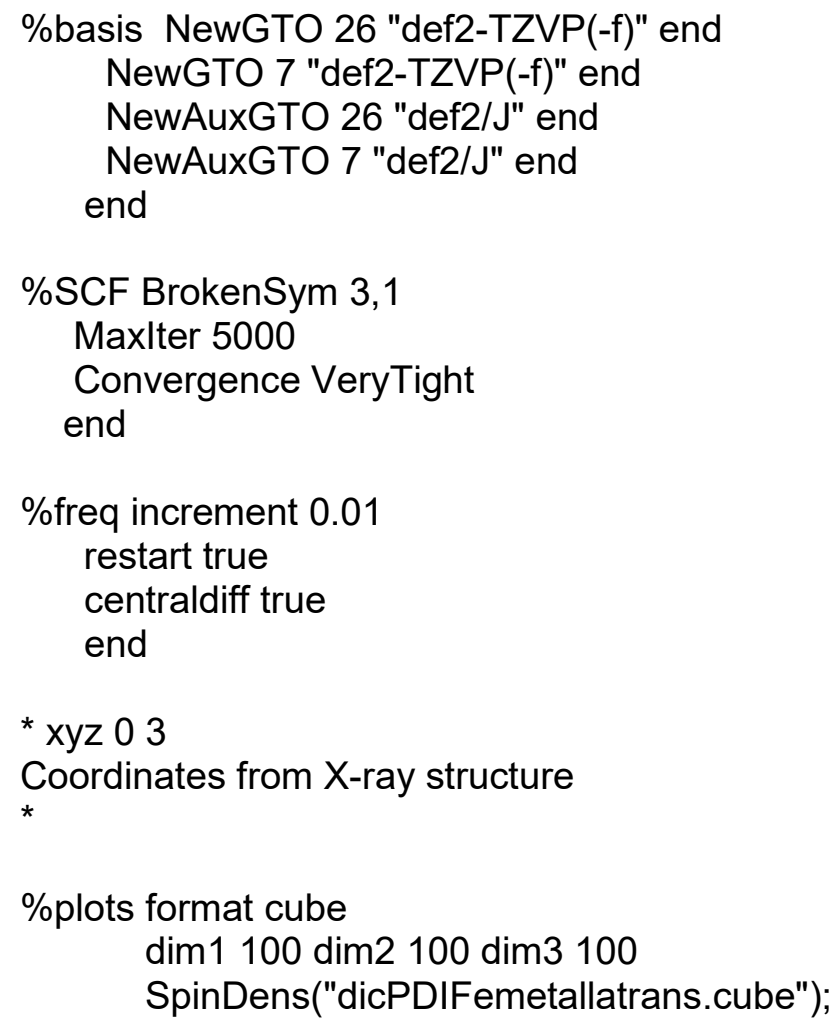

\#for ${ }^{57} \mathrm{Fe}$ Mössbauer parameters add \%eprnmr nuclei = all Fe $\{$ rho, fgrad $\}$ instead of \%plots 
end

- Calculated Thermochemical Properties - (dicPDI)Fe(trans-bimetallacyclo[4.3.0]nonane) (Calculated at $298.15 \mathrm{~K}$ )

Final Gibbs free enthalpy $\quad$... $\quad-3331.636839456332$

n Calculated ${ }^{57} \mathrm{Fe}$ Mössbauer Properties - (dicPDI)Fe(trans-bimetallacyclo[4.3.0]nonane)

$\rho(0)=11582.607077441 \times 10^{-3}-->0.341 \mathrm{~mm} / \mathrm{s}$

$\Delta \mathrm{E}=-2.138 \mathrm{~mm} / \mathrm{s}$

- Optimized Coordinates - (dicPDI)Fe(trans-bimetallacyclo[4.3.0]nonane)

$\begin{array}{lrrr}\mathrm{C} & -2.71662 & 3.18632 & 1.28965 \\ \mathrm{H} & -3.65482 & 2.67910 & 1.54083 \\ \mathrm{H} & -2.92887 & 3.94925 & 0.52588 \\ \mathrm{H} & -2.36534 & 3.72189 & 2.18793 \\ \mathrm{C} & -1.65912 & 2.20612 & 0.83370 \\ \mathrm{C} & -0.36240 & 2.72268 & 0.40179 \\ \mathrm{C} & -0.04570 & 4.08179 & 0.38865 \\ \mathrm{H} & -0.79652 & 4.82159 & 0.66754 \\ \mathrm{C} & 1.24762 & 4.49842 & 0.03870 \\ \mathrm{H} & 1.50611 & 5.55874 & 0.00586 \\ \mathrm{C} & 2.21781 & 3.52095 & -0.19744 \\ \mathrm{H} & 3.25004 & 3.81382 & -0.39357 \\ \mathrm{C} & 1.86912 & 2.16784 & -0.15522 \\ \mathrm{C} & 2.83667 & 1.08629 & -0.20353 \\ \mathrm{C} & 4.30283 & 1.40297 & -0.37864 \\ \mathrm{H} & 4.91580 & 0.49560 & -0.33977 \\ \mathrm{H} & 4.65001 & 2.08165 & 0.41666 \\ \mathrm{H} & 4.49287 & 1.90921 & -1.33865 \\ \mathrm{C} & 3.22282 & -1.25049 & 0.04113 \\ \mathrm{C} & 3.59555 & -1.74801 & 1.31830 \\ \mathrm{C} & 4.40172 & -2.89300 & 1.38263 \\ \mathrm{H} & 4.70141 & -3.28153 & 2.36046 \\ \mathrm{C} & 4.84173 & -3.53767 & 0.22730 \\ \mathrm{H} & 5.46901 & -4.42999 & 0.29868 \\ \mathrm{C} & 4.48015 & -3.02900 & -1.01867 \\ \mathrm{H} & 4.83760 & -3.52975 & -1.92299 \\ \mathrm{C} & 3.67781 & -1.88368 & -1.14742 \\ \mathrm{C} & 3.20057 & -1.03315 & 2.60227 \\ \mathrm{H} & 2.48374 & -0.24341 & 2.33920 \\ \mathrm{C} & 2.53548 & -1.92213 & 3.70634 \\ \mathrm{H} & 1.45428 & -1.71606 & 3.73776 \\ \mathrm{H} & 2.63931 & -2.99557 & 3.48948 \\ \mathrm{C} & 3.21191 & -1.54354 & 5.04790 \\ \mathrm{H} & 2.50229 & -1.51903 & 5.89089 \\ \mathrm{H} & 3.98389 & -2.28834 & 5.30692 \\ \mathrm{C} & 3.88976 & -0.18817 & 4.77819 \\ \mathrm{H} & 4.69319 & 0.05089 & 5.49375\end{array}$




\begin{tabular}{|c|c|c|c|}
\hline $\mathrm{H}$ & 3.14842 & 0.62826 & 4.83973 \\
\hline C & 4.38573 & -0.34099 & 3.33397 \\
\hline $\mathrm{H}$ & 5.27410 & -0.99580 & 3.31397 \\
\hline $\mathrm{H}$ & 4.67890 & 0.60906 & 2.85840 \\
\hline C & -3.06220 & 0.32541 & 1.18075 \\
\hline C & -3.29964 & -0.14871 & 2.49556 \\
\hline C & -4.55700 & -0.69504 & 2.79722 \\
\hline $\mathrm{H}$ & -4.75334 & -1.06045 & 3.80854 \\
\hline C & -5.56339 & -0.77864 & 1.84039 \\
\hline $\mathrm{H}$ & -6.53808 & -1.19915 & 2.10196 \\
\hline C & -5.30772 & -0.33719 & 0.54378 \\
\hline $\mathrm{H}$ & -6.09655 & -0.41910 & -0.20767 \\
\hline C & -4.06640 & 0.20651 & 0.17839 \\
\hline C & -3.84688 & 0.67102 & -1.25489 \\
\hline $\mathrm{H}$ & -2.76610 & 0.80036 & -1.40485 \\
\hline C & -4.51316 & 2.01616 & -1.63710 \\
\hline $\mathrm{H}$ & -5.57568 & 2.00936 & -1.33192 \\
\hline $\mathrm{H}$ & -4.04295 & 2.88253 & -1.14954 \\
\hline C & -4.38460 & 2.04634 & -3.16506 \\
\hline $\mathrm{H}$ & -5.06384 & 2.76942 & -3.64270 \\
\hline $\mathrm{H}$ & -3.35700 & 2.34457 & -3.43568 \\
\hline C & -4.64998 & 0.58776 & -3.60719 \\
\hline $\mathrm{H}$ & -5.69877 & 0.46495 & -3.92426 \\
\hline $\mathrm{H}$ & -4.03328 & 0.30606 & -4.47447 \\
\hline C & -4.35304 & -0.29506 & -2.36421 \\
\hline $\mathrm{H}$ & -3.61304 & -1.07942 & -2.57213 \\
\hline $\mathrm{H}$ & -5.26822 & -0.81434 & -2.04268 \\
\hline C & -2.25346 & -0.06826 & 3.59132 \\
\hline $\mathrm{H}$ & -1.38174 & 0.47715 & 3.19916 \\
\hline C & -1.75210 & -1.44619 & 4.11812 \\
\hline $\mathrm{H}$ & -2.55725 & -2.19406 & 4.04481 \\
\hline $\mathrm{H}$ & -0.92654 & -1.82747 & 3.49958 \\
\hline C & -1.34105 & -1.21934 & 5.60121 \\
\hline $\mathrm{H}$ & -0.28965 & -1.48689 & 5.79164 \\
\hline $\mathrm{H}$ & -1.94519 & -1.85718 & 6.26560 \\
\hline C & -1.62040 & 0.27312 & 5.89471 \\
\hline $\mathrm{H}$ & -1.92371 & 0.46031 & 6.93761 \\
\hline $\mathrm{H}$ & -0.71325 & 0.87298 & 5.70643 \\
\hline C & -2.70207 & 0.65702 & 4.88106 \\
\hline $\mathrm{H}$ & -2.81665 & 1.74524 & 4.74658 \\
\hline $\mathrm{H}$ & -3.68050 & 0.26770 & 5.21366 \\
\hline C & -0.26536 & -2.11664 & 0.51691 \\
\hline $\mathrm{H}$ & 0.52943 & -2.37602 & 1.22628 \\
\hline $\mathrm{H}$ & -1.22771 & -2.05641 & 1.03851 \\
\hline C & -0.52682 & -0.90992 & -1.86566 \\
\hline $\mathrm{H}$ & -1.13431 & -0.15926 & -2.37874 \\
\hline $\mathrm{H}$ & 0.42844 & -0.99007 & -2.39461 \\
\hline$N$ & -1.79253 & 0.90711 & 0.85866 \\
\hline $\mathrm{N}$ & 0.56066 & 1.77320 & 0.02765 \\
\hline $\mathrm{N}$ & 2.35651 & -0.11691 & -0.00750 \\
\hline $\mathrm{Fe}$ & 0.08495 & -0.18396 & -0.03916 \\
\hline C & -1.19225 & -2.27860 & -1.72391 \\
\hline
\end{tabular}




$\begin{array}{lrrr}\mathrm{H} & -2.19089 & -2.13036 & -1.27120 \\ \mathrm{C} & -0.36449 & -3.04660 & -0.68505 \\ \mathrm{H} & 0.64919 & -3.18780 & -1.10716 \\ \mathrm{C} & -0.94930 & -4.43982 & -0.39860 \\ \mathrm{H} & -0.30204 & -4.99547 & 0.30213 \\ \mathrm{H} & -1.92528 & -4.31876 & 0.10823 \\ \mathrm{C} & -1.14916 & -5.24578 & -1.69185 \\ \mathrm{H} & -0.16263 & -5.48794 & -2.12935 \\ \mathrm{H} & -1.63148 & -6.21192 & -1.45994 \\ \mathrm{C} & -1.97926 & -4.47547 & -2.72885 \\ \mathrm{H} & -3.01206 & -4.35876 & -2.34964 \\ \mathrm{H} & -2.05497 & -5.06013 & -3.66225 \\ \mathrm{C} & -1.39295 & -3.08359 & -3.01971 \\ \mathrm{H} & -0.41996 & -3.19813 & -3.53195 \\ \mathrm{H} & -2.04511 & -2.53236 & -3.72045 \\ \mathrm{C} & 3.37612 & -1.35349 & -2.54418 \\ \mathrm{H} & 2.66050 & -0.52405 & -2.44929 \\ \mathrm{C} & 2.75957 & -2.38938 & -3.54266 \\ \mathrm{H} & 2.93701 & -3.42371 & -3.21296 \\ \mathrm{H} & 1.66835 & -2.26189 & -3.57866 \\ \mathrm{C} & 3.41059 & -2.12005 & -4.92600 \\ \mathrm{H} & 2.67921 & -2.12998 & -5.74975 \\ \mathrm{H} & 4.15237 & -2.90375 & -5.15565 \\ \mathrm{C} & 4.13172 & -0.76867 & -4.77204 \\ \mathrm{H} & 3.41954 & 0.06268 & -4.91685 \\ \mathrm{H} & 4.94921 & -0.62164 & -5.49646 \\ \mathrm{C} & 4.60635 & -0.79778 & -3.31438 \\ \mathrm{H} & 4.94363 & 0.18012 & -2.93935 \\ \mathrm{H} & 5.45924 & -1.49185 & -3.21228\end{array}$

- DFT Input File - (dicPDI)Fe(cis-bimetallacyclo[4.3.0]nonane)

! UKS B3LYP RIJCOSX def2-SVP def2/J Normalprint SlowConv TightSCF TightOpt NumFreq Grid5 FinalGrid6 GridX4 Pal8 UCO

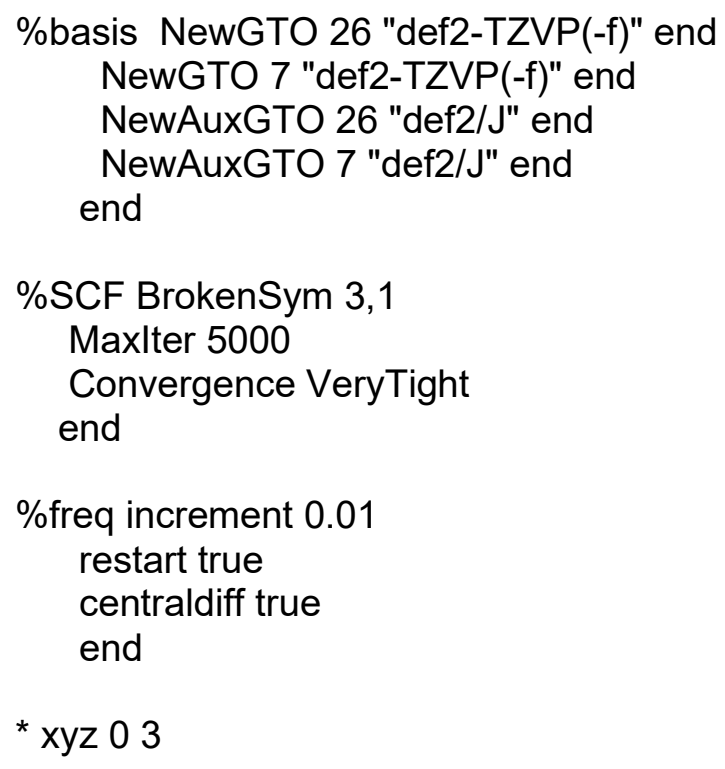


Coordinates from X-ray structure with altered bimetallacycle to cis configuration

\%plots format cube

$\operatorname{dim} 1100 \operatorname{dim} 2100 \operatorname{dim} 3100$

SpinDens("dicPDIFemetallacis.cube");

end

- Calculated Thermochemical Properties - (dicPDI)Fe(cis-bimetallacyclo[4.3.0]nonane) (Calculated at $298.15 \mathrm{~K}$ )

Final Gibbs free enthalpy $\quad$... $\quad-3331.630874483051$

- Optimized Coordinates - (dicPDI)Fe(trans-bimetallacyclo[4.3.0]nonane)

$\begin{array}{lrrr}\mathrm{C} & -2.71662 & 3.18632 & 1.28965 \\ \mathrm{H} & -3.65482 & 2.67910 & 1.54083 \\ \mathrm{H} & -2.92887 & 3.94925 & 0.52588 \\ \mathrm{H} & -2.36534 & 3.72189 & 2.18793 \\ \mathrm{C} & -1.65912 & 2.20612 & 0.83370 \\ \mathrm{C} & -0.36240 & 2.72268 & 0.40179 \\ \mathrm{C} & -0.04570 & 4.08179 & 0.38865 \\ \mathrm{H} & -0.79652 & 4.82159 & 0.66754 \\ \mathrm{C} & 1.24762 & 4.49842 & 0.03870 \\ \mathrm{H} & 1.50611 & 5.55874 & 0.00586 \\ \mathrm{C} & 2.21781 & 3.52095 & -0.19744 \\ \mathrm{H} & 3.25004 & 3.81382 & -0.39357 \\ \mathrm{C} & 1.86912 & 2.16784 & -0.15522 \\ \mathrm{C} & 2.83667 & 1.08629 & -0.20353 \\ \mathrm{C} & 4.30283 & 1.40297 & -0.37864 \\ \mathrm{H} & 4.91580 & 0.49560 & -0.33977 \\ \mathrm{H} & 4.65001 & 2.08165 & 0.41666 \\ \mathrm{H} & 4.49287 & 1.90921 & -1.33865 \\ \mathrm{C} & 3.22282 & -1.25049 & 0.04113 \\ \mathrm{C} & 3.59555 & -1.74801 & 1.31830 \\ \mathrm{C} & 4.40172 & -2.89300 & 1.38263 \\ \mathrm{H} & 4.70141 & -3.28153 & 2.36046 \\ \mathrm{C} & 4.84173 & -3.53767 & 0.22730 \\ \mathrm{H} & 5.46901 & -4.42999 & 0.29868 \\ \mathrm{C} & 4.48015 & -3.02900 & -1.01867 \\ \mathrm{H} & 4.83760 & -3.52975 & -1.92299 \\ \mathrm{C} & 3.67781 & -1.88368 & -1.14742 \\ \mathrm{C} & 3.20057 & -1.03315 & 2.60227 \\ \mathrm{H} & 2.48374 & -0.24341 & 2.33920 \\ \mathrm{C} & 2.53548 & -1.92213 & 3.70634 \\ \mathrm{H} & 1.45428 & -1.71606 & 3.73776 \\ \mathrm{H} & 2.63931 & -2.99557 & 3.48948 \\ \mathrm{C} & 3.21191 & -1.54354 & 5.04790 \\ \mathrm{H} & 2.50229 & -1.51903 & 5.89089 \\ \mathrm{H} & 3.98389 & -2.28834 & 5.30692 \\ \mathrm{C} & 3.88976 & -0.18817 & 4.77819\end{array}$




\begin{tabular}{|c|c|c|c|}
\hline $\mathrm{H}$ & 4.69319 & 0.05089 & 5.49375 \\
\hline $\mathrm{H}$ & 3.14842 & 0.62826 & 4.83973 \\
\hline C & 4.38573 & -0.34099 & 3.33397 \\
\hline $\mathrm{H}$ & 5.27410 & -0.99580 & 3.31397 \\
\hline $\mathrm{H}$ & 4.67890 & 0.60906 & 2.85840 \\
\hline C & -3.06220 & 0.32541 & 1.18075 \\
\hline C & -3.29964 & -0.14871 & 2.49556 \\
\hline C & -4.55700 & -0.69504 & 2.79722 \\
\hline $\mathrm{H}$ & -4.75334 & -1.06045 & 3.80854 \\
\hline C & -5.56339 & -0.77864 & 1.84039 \\
\hline $\mathrm{H}$ & -6.53808 & -1.19915 & 2.10196 \\
\hline C & -5.30772 & -0.33719 & 0.54378 \\
\hline $\mathrm{H}$ & -6.09655 & -0.41910 & -0.20767 \\
\hline C & -4.06640 & 0.20651 & 0.17839 \\
\hline C & -3.84688 & 0.67102 & -1.25489 \\
\hline $\mathrm{H}$ & -2.76610 & 0.80036 & -1.40485 \\
\hline C & -4.51316 & 2.01616 & -1.63710 \\
\hline $\mathrm{H}$ & -5.57568 & 2.00936 & -1.33192 \\
\hline $\mathrm{H}$ & -4.04295 & 2.88253 & -1.14954 \\
\hline C & -4.38460 & 2.04634 & -3.16506 \\
\hline $\mathrm{H}$ & -5.06384 & 2.76942 & -3.64270 \\
\hline $\mathrm{H}$ & -3.35700 & 2.34457 & -3.43568 \\
\hline C & -4.64998 & 0.58776 & -3.60719 \\
\hline $\mathrm{H}$ & -5.69877 & 0.46495 & -3.92426 \\
\hline $\mathrm{H}$ & -4.03328 & 0.30606 & -4.47447 \\
\hline C & -4.35304 & -0.29506 & -2.36421 \\
\hline $\mathrm{H}$ & -3.61304 & -1.07942 & -2.57213 \\
\hline $\mathrm{H}$ & -5.26822 & -0.81434 & -2.04268 \\
\hline C & -2.25346 & -0.06826 & 3.59132 \\
\hline $\mathrm{H}$ & -1.38174 & 0.47715 & 3.19916 \\
\hline C & -1.75210 & -1.44619 & 4.11812 \\
\hline $\mathrm{H}$ & -2.55725 & -2.19406 & 4.04481 \\
\hline $\mathrm{H}$ & -0.92654 & -1.82747 & 3.49958 \\
\hline C & -1.34105 & -1.21934 & 5.60121 \\
\hline $\mathrm{H}$ & -0.28965 & -1.48689 & 5.79164 \\
\hline $\mathrm{H}$ & -1.94519 & -1.85718 & 6.26560 \\
\hline C & -1.62040 & 0.27312 & 5.89471 \\
\hline $\mathrm{H}$ & -1.92371 & 0.46031 & 6.93761 \\
\hline $\mathrm{H}$ & -0.71325 & 0.87298 & 5.70643 \\
\hline C & -2.70207 & 0.65702 & 4.88106 \\
\hline $\mathrm{H}$ & -2.81665 & 1.74524 & 4.74658 \\
\hline $\mathrm{H}$ & -3.68050 & 0.26770 & 5.21366 \\
\hline C & -0.26536 & -2.11664 & 0.51691 \\
\hline $\mathrm{H}$ & 0.52943 & -2.37602 & 1.22628 \\
\hline $\mathrm{H}$ & -1.22771 & -2.05641 & 1.03851 \\
\hline C & -0.52682 & -0.90992 & -1.86566 \\
\hline $\mathrm{H}$ & -1.13431 & -0.15926 & -2.37874 \\
\hline $\mathrm{H}$ & 0.42844 & -0.99007 & -2.39461 \\
\hline$N$ & -1.79253 & 0.90711 & 0.85866 \\
\hline$N$ & 0.56066 & 1.77320 & 0.02765 \\
\hline $\mathrm{N}$ & 2.35651 & -0.11691 & -0.00750 \\
\hline $\mathrm{Fe}$ & 0.08495 & -0.18396 & -0.03916 \\
\hline
\end{tabular}




$\begin{array}{lrrr}\mathrm{C} & -1.19225 & -2.27860 & -1.72391 \\ \mathrm{H} & -2.19089 & -2.13036 & -1.27120 \\ \mathrm{C} & -0.36449 & -3.04660 & -0.68505 \\ \mathrm{H} & 0.64919 & -3.18780 & -1.10716 \\ \mathrm{C} & -0.94930 & -4.43982 & -0.39860 \\ \mathrm{H} & -0.30204 & -4.99547 & 0.30213 \\ \mathrm{H} & -1.92528 & -4.31876 & 0.10823 \\ \mathrm{C} & -1.14916 & -5.24578 & -1.69185 \\ \mathrm{H} & -0.16263 & -5.48794 & -2.12935 \\ \mathrm{H} & -1.63148 & -6.21192 & -1.45994 \\ \mathrm{C} & -1.97926 & -4.47547 & -2.72885 \\ \mathrm{H} & -3.01206 & -4.35876 & -2.34964 \\ \mathrm{H} & -2.05497 & -5.06013 & -3.66225 \\ \mathrm{C} & -1.39295 & -3.08359 & -3.01971 \\ \mathrm{H} & -0.41996 & -3.19813 & -3.53195 \\ \mathrm{H} & -2.04511 & -2.53236 & -3.72045 \\ \mathrm{C} & 3.37612 & -1.35349 & -2.54418 \\ \mathrm{H} & 2.66050 & -0.52405 & -2.44929 \\ \mathrm{C} & 2.75957 & -2.38938 & -3.54266 \\ \mathrm{H} & 2.93701 & -3.42371 & -3.21296 \\ \mathrm{H} & 1.66835 & -2.26189 & -3.57866 \\ \mathrm{C} & 3.41059 & -2.12005 & -4.92600 \\ \mathrm{H} & 2.67921 & -2.12998 & -5.74975 \\ \mathrm{H} & 4.15237 & -2.90375 & -5.15565 \\ \mathrm{C} & 4.13172 & -0.76867 & -4.77204 \\ \mathrm{H} & 3.41954 & 0.06268 & -4.91685 \\ \mathrm{H} & 4.94921 & -0.62164 & -5.49646 \\ \mathrm{C} & 4.60635 & -0.79778 & -3.31438 \\ \mathrm{H} & 4.94363 & 0.18012 & -2.93935 \\ \mathrm{H} & 5.45924 & -1.49185 & -3.21228\end{array}$

- DFT Input File - (dicPDI)Fe(trans-2,3-dimethylmetallacyclopentane)

! UKS B3LYP RIJCOSX def2-SVP def2/J Normalprint SlowConv TightSCF Opt NumFreq Pal8 UCO

\%basis NewGTO 26 "def2-TZVP(-f)" end NewGTO 7 "def2-TZVP(-f)" end NewAuxGTO 26 "def2/J" end NewAuxGTO 7 "def2/J" end end

\%SCF BrokenSym 3,1 Maxiter 5000

TolE 1e-7

TolErr 1e-6 end

\%freq increment 0.01 restart true centraldiff true end 


\begin{tabular}{|c|c|c|c|}
\hline C & 3.90325 & -0.11848 & -1.49066 \\
\hline C & 2.51308 & -0.25168 & -1.38521 \\
\hline $\mathrm{N}$ & 1.87648 & -0.09364 & -0.17017 \\
\hline C & 2.58882 & 0.41547 & 0.88837 \\
\hline C & 3.96972 & 0.58706 & 0.81119 \\
\hline C & 4.64943 & 0.27737 & -0.38069 \\
\hline C & 1.62875 & -0.45250 & -2.51477 \\
\hline C & 1.79374 & 0.77954 & 2.06579 \\
\hline C & 2.19466 & -0.65628 & -3.89980 \\
\hline$N$ & 0.34374 & -0.37258 & -2.24700 \\
\hline C & 2.49894 & 1.28714 & 3.30239 \\
\hline$N$ & 0.50174 & 0.67076 & 1.94671 \\
\hline C & -0.64188 & -0.39526 & -3.28140 \\
\hline C & -0.37117 & 0.93241 & 3.04866 \\
\hline C & -1.19502 & -1.61547 & -3.75409 \\
\hline C & -2.26450 & -1.54998 & -4.66177 \\
\hline C & -2.76891 & -0.33459 & -5.11845 \\
\hline C & -2.17517 & 0.85208 & -4.69164 \\
\hline C & -1.10531 & 0.85055 & -3.78601 \\
\hline C & -0.50134 & 0.00825 & 4.12529 \\
\hline C & -1.40198 & 0.31882 & 5.15858 \\
\hline C & -2.16376 & 1.48493 & 5.14798 \\
\hline C & -2.03580 & 2.37084 & 4.08146 \\
\hline C & -1.15221 & 2.11834 & 3.02257 \\
\hline C & -0.62904 & -2.98036 & -3.38602 \\
\hline C & -0.42967 & 2.16715 & -3.42969 \\
\hline C & -1.00045 & 3.15812 & 1.92744 \\
\hline $\mathrm{C}$ & 0.31397 & -1.27696 & 4.23309 \\
\hline $\mathrm{Fe}$ & -0.10473 & -0.42378 & -0.06466 \\
\hline C & -2.12250 & -0.20155 & -0.03842 NewGTO "def2-TZVP(-f)" end \\
\hline C & -2.89628 & -1.50994 & 0.12349 \\
\hline C & -2.06652 & -2.35504 & 1.10762 \\
\hline C & -0.62012 & -2.29125 & 0.60199 NewGTO "def2-TZVP(-f)" end \\
\hline C & -4.35750 & -1.28812 & 0.54023 \\
\hline C & -2.57863 & -3.78744 & 1.32342 \\
\hline C & -1.62962 & -4.02823 & -2.84631 \\
\hline C & -0.84963 & -5.34455 & -2.94542 \\
\hline C & -0.14968 & -5.25007 & -4.31876 \\
\hline C & 0.30751 & 2.84263 & -4.62001 \\
\hline C & 0.46665 & 4.30017 & -4.16821 \\
\hline C & -0.90427 & 4.61747 & -3.53986 \\
\hline C & -1.36054 & 3.29355 & -2.87081 \\
\hline C & -2.31782 & 3.63214 & 1.24617 \\
\hline C & -0.62370 & 5.44136 & 1.23904 \\
\hline C & -0.35662 & 4.48367 & 2.40238 \\
\hline C & 1.36155 & -1.26774 & 5.41042 \\
\hline C & 0.91461 & -2.36550 & 6.39102 \\
\hline C & 0.27119 & -3.41259 & 5.47711 \\
\hline C & -0.54197 & -2.55982 & 4.49163 \\
\hline C & 0.04580 & -3.73044 & -4.58338 \\
\hline C & -2.07036 & 5.10444 & 0.80206 \\
\hline
\end{tabular}




\begin{tabular}{|c|c|c|c|}
\hline $\mathrm{H}$ & 0.13283 & -2.83789 & -2.60478 \\
\hline $\mathrm{H}$ & -2.21626 & 5.24650 & -0.28065 \\
\hline $\mathrm{H}$ & 4.39698 & -0.30160 & -2.44620 \\
\hline $\mathrm{H}$ & 4.52402 & 0.97910 & 1.66459 \\
\hline & 5.73190 & 0.40071 & -0.45245 \\
\hline & 2.77296 & -1.59312 & -3.96402 \\
\hline & 2.87840 & 0.16350 & -4.17254 \\
\hline & 1.39696 & -0.69346 & -4.65107 \\
\hline $\mathrm{H}$ & 3.14081 & 0.51053 & 3.74693 \\
\hline $\mathrm{H}$ & 1.78008 & 1.61605 & 4.06122 \\
\hline $\mathrm{H}$ & 3.14928 & 2.14016 & 3.05105 \\
\hline $\mathrm{H}$ & -2.70726 & -2.47969 & -5.02901 \\
\hline $\mathrm{H}$ & -3.60920 & -0.31239 & -5.81748 \\
\hline 卜 & -2.54555 & 1.80594 & -5.0790 \\
\hline & -1.50614 & -0.37151 & 5.99975 \\
\hline $\mathrm{H}$ & -2.85123 & 1.70590 & 5.96889 \\
\hline $\mathrm{H}$ & -2.62391 & 3.29172 & 4.07728 \\
\hline $\mathrm{H}$ & 0.33009 & 1.96217 & -2.66416 \\
\hline $\mathrm{H}$ & -0.34823 & 2.74297 & 1.14423 \\
\hline $\mathrm{H}$ & 0.86020 & -1.41477 & 3.29157 \\
\hline $\mathrm{H}$ & -2.26737 & 0.43241 & 0.84416 \\
\hline $\mathrm{H}$ & -2.38446 & 0.35910 & -0.94385 \\
\hline $\mathrm{H}$ & -2.89383 & -2.03734 & -0.84806 \\
\hline $\mathrm{H}$ & -2.10620 & -1.83007 & 2.07949 \\
\hline $\mathrm{H}$ & -0.48820 & -2.90083 & -0.29873 \\
\hline $\mathrm{H}$ & 0.12069 & -2.61793 & 1.33837 \\
\hline $\mathrm{H}$ & -4.41031 & -0.73682 & 1.49548 \\
\hline $\mathrm{H}$ & -4.89976 & -0.69334 & -0.2131 \\
\hline $\mathrm{H}$ & -4.90534 & -2.23663 & 0.66980 \\
\hline $\mathrm{H}$ & -1.88127 & -4.37285 & 1.94495 \\
\hline $\mathrm{H}$ & -3.56024 & -3.80803 & 1.8266 \\
\hline $\mathrm{H}$ & -2.68480 & -4.32091 & 0.36359 \\
\hline $\mathrm{H}$ & -1.97950 & -3.79784 & -1.8306 \\
\hline $\mathrm{H}$ & -2.52271 & -4.07515 & -3.4923 \\
\hline $\mathrm{H}$ & -1.48942 & -6.23588 & -2.8427 \\
\hline $\mathrm{H}$ & -0.09897 & -5.39337 & -2.1366 \\
\hline $\mathrm{H}$ & 0.79851 & -5.80879 & -4.34832 \\
\hline $\mathrm{H}$ & -0.79363 & -5.69191 & -5.09705 \\
\hline $\mathrm{H}$ & -0.43490 & -3.43575 & -5.52847 \\
\hline $\mathrm{H}$ & 1.10711 & -3.45534 & -4.6801 \\
\hline $\mathrm{H}$ & -0.31920 & 2.79809 & -5.52706 \\
\hline $\mathrm{H}$ & 1.25971 & 2.34529 & -4.86699 \\
\hline $\mathrm{H}$ & 1.26335 & 4.37345 & -3.40696 \\
\hline $\mathrm{H}$ & 0.73978 & 4.98456 & -4.98791 \\
\hline $\mathrm{H}$ & -1.61052 & 4.90083 & -4.33829 \\
\hline $\mathrm{H}$ & -0.87130 & 5.46335 & -2.83361 \\
\hline $\mathrm{H}$ & -1.26476 & 3.34235 & -1.77516 \\
\hline $\mathrm{H}$ & -2.42060 & 3.08606 & -3.0793 \\
\hline $\mathrm{H}$ & -3.15861 & 3.57776 & 1.95496 \\
\hline $\mathrm{H}$ & -2.58621 & 2.97381 & 0.40650 \\
\hline $\mathrm{H}$ & -2.78358 & 5.77877 & 1.30098 \\
\hline $\mathrm{H}$ & 0.08124 & 5.22501 & 0.41791 \\
\hline
\end{tabular}




$\begin{array}{lrrr}\mathrm{H} & 0.71043 & 4.37888 & 2.65916 \\ \mathrm{H} & -0.87459 & 4.83950 & 3.31114 \\ \mathrm{H} & 1.44797 & -0.28104 & 5.88902 \\ \mathrm{H} & 2.35820 & -1.52084 & 5.01134 \\ \mathrm{H} & 1.74273 & -2.76092 & 7.00151 \\ \mathrm{H} & 0.15785 & -1.96627 & 7.09056 \\ \mathrm{H} & 1.05790 & -3.97400 & 4.94100 \\ \mathrm{H} & -0.34383 & -4.15076 & 6.01685 \\ \mathrm{H} & -0.78130 & -3.09080 & 3.56075 \\ \mathrm{H} & -1.50636 & -2.28795 & 4.94919 \\ \mathrm{H} & -0.48864 & 6.50058 & 1.51328 \\ * & & & \end{array}$

\%plots format cube $\operatorname{dim} 1100 \operatorname{dim} 2100 \operatorname{dim} 3100$

SpinDens("dicPDIFemetallacycle.cube");

end

Calculated $\quad$ Thermochemical $\quad$ Properties
dimethylmetallacyclopentane) (Calculated at 298.15 K)

Final Gibbs free enthalpy $\ldots-3254.502455865792$

- Optimized Coordiantes - (dicPDI)Fe(trans-2,3-dimethylmetallacyclopentane)

$\begin{array}{lrrr}\text { C } & 3.90325 & -0.11848 & -1.49066 \\ \text { C } & 2.51308 & -0.25168 & -1.38521 \\ \text { N } & 1.87648 & -0.09364 & -0.17017 \\ \text { C } & 2.58882 & 0.41547 & 0.88837 \\ \text { C } & 3.96972 & 0.58706 & 0.81119 \\ \text { C } & 4.64943 & 0.27737 & -0.38069 \\ \text { C } & 1.62875 & -0.45250 & -2.51477 \\ \text { C } & 1.79374 & 0.77954 & 2.06579 \\ \text { C } & 2.19466 & -0.65628 & -3.89980 \\ \text { N } & 0.34374 & -0.37258 & -2.24700 \\ \text { C } & 2.49894 & 1.28714 & 3.30239 \\ \text { N } & 0.50174 & 0.67076 & 1.94671 \\ \text { C } & -0.64188 & -0.39526 & -3.28140 \\ \text { C } & -0.37117 & 0.93241 & 3.04866 \\ \text { C } & -1.19502 & -1.61547 & -3.75409 \\ \text { C } & -2.26450 & -1.54998 & -4.66177 \\ \text { C } & -2.76891 & -0.33459 & -5.11845 \\ \text { C } & -2.17517 & 0.85208 & -4.69164 \\ \text { C } & -1.10531 & 0.85055 & -3.78601 \\ \text { C } & -0.50134 & 0.00825 & 4.12529 \\ \text { C } & -1.40198 & 0.31882 & 5.15858 \\ \text { C } & -2.16376 & 1.48493 & 5.14798 \\ \text { C } & -2.03580 & 2.37084 & 4.08146 \\ \text { C } & -1.15221 & 2.11834 & 3.02257 \\ \text { C } & -0.62904 & -2.98036 & -3.38602 \\ \text { C } & -0.42967 & 2.16715 & -3.42969 \\ \text { C } & -1.00045 & 3.15812 & 1.92744\end{array}$




\begin{tabular}{|c|c|c|c|}
\hline $\mathrm{C}$ & 0.31397 & -1.27696 & 4.23309 \\
\hline $\mathrm{Fe}$ & -0.10473 & -0.42378 & -0.06466 \\
\hline C & -2.12250 & -0.20155 & -0.03842 \\
\hline C & -2.89628 & -1.50994 & 0.12349 \\
\hline C & -2.06652 & -2.35504 & 1.10762 \\
\hline C & -0.62012 & -2.29125 & 0.60199 \\
\hline C & -4.35750 & -1.28812 & 0.54023 \\
\hline C & -2.57863 & -3.78744 & 1.32342 \\
\hline C & -1.62962 & -4.02823 & -2.84631 \\
\hline C & -0.84963 & -5.34455 & -2.94542 \\
\hline C & -0.14968 & -5.25007 & -4.31876 \\
\hline C & 0.30751 & 2.84263 & -4.62001 \\
\hline C & 0.46665 & 4.30017 & -4.16821 \\
\hline C & -0.90427 & 4.61747 & -3.53986 \\
\hline C & -1.36054 & 3.29355 & -2.87081 \\
\hline C & -2.31782 & 3.63214 & 1.24617 \\
\hline C & -0.62370 & 5.44136 & 1.23904 \\
\hline C & -0.35662 & 4.48367 & 2.40238 \\
\hline C & 1.36155 & -1.26774 & 5.41042 \\
\hline C & 0.91461 & -2.36550 & 6.39102 \\
\hline C & 0.27119 & -3.41259 & 5.47711 \\
\hline C & -0.54197 & -2.55982 & 4.49163 \\
\hline C & 0.04580 & -3.73044 & -4.58338 \\
\hline C & -2.07036 & 5.10444 & 0.80206 \\
\hline $\mathrm{H}$ & 0.13283 & -2.83789 & -2.60478 \\
\hline $\mathrm{H}$ & -2.21626 & 5.24650 & -0.28065 \\
\hline $\mathrm{H}$ & 4.39698 & -0.30160 & -2.44620 \\
\hline $\mathrm{H}$ & 4.52402 & 0.97910 & 1.66459 \\
\hline $\mathrm{H}$ & 5.73190 & 0.40071 & -0.45245 \\
\hline $\mathrm{H}$ & 2.77296 & -1.59312 & -3.96402 \\
\hline $\mathrm{H}$ & 2.87840 & 0.16350 & -4.17254 \\
\hline $\mathrm{H}$ & 1.39696 & -0.69346 & -4.65107 \\
\hline $\mathrm{H}$ & 3.14081 & 0.51053 & 3.74693 \\
\hline $\mathrm{H}$ & 1.78008 & 1.61605 & 4.06122 \\
\hline $\mathrm{H}$ & 3.14928 & 2.14016 & 3.05105 \\
\hline $\mathrm{H}$ & -2.70726 & -2.47969 & -5.02901 \\
\hline $\mathrm{H}$ & -3.60920 & -0.31239 & -5.81748 \\
\hline $\mathrm{H}$ & -2.54555 & 1.80594 & -5.07905 \\
\hline $\mathrm{H}$ & -1.50614 & -0.37151 & 5.99975 \\
\hline $\mathrm{H}$ & -2.85123 & 1.70590 & 5.96889 \\
\hline $\mathrm{H}$ & -2.62391 & 3.29172 & 4.07728 \\
\hline $\mathrm{H}$ & 0.33009 & 1.96217 & -2.66416 \\
\hline $\mathrm{H}$ & -0.34823 & 2.74297 & 1.14423 \\
\hline $\mathrm{H}$ & 0.86020 & -1.41477 & 3.29157 \\
\hline $\mathrm{H}$ & -2.26737 & 0.43241 & 0.84416 \\
\hline $\mathrm{H}$ & -2.38446 & 0.35910 & -0.94385 \\
\hline $\mathrm{H}$ & -2.89383 & -2.03734 & -0.84806 \\
\hline $\mathrm{H}$ & -2.10620 & -1.83007 & 2.07949 \\
\hline $\mathrm{H}$ & -0.48820 & -2.90083 & -0.29873 \\
\hline $\mathrm{H}$ & 0.12069 & -2.61793 & 1.33837 \\
\hline $\mathrm{H}$ & -4.41031 & -0.73682 & 1.49548 \\
\hline $\mathrm{H}$ & -4.89976 & -0.69334 & -0.21317 \\
\hline
\end{tabular}




$\begin{array}{rrrr}\mathrm{H} & -4.90534 & -2.23663 & 0.66980 \\ \mathrm{H} & -1.88127 & -4.37285 & 1.94495 \\ \mathrm{H} & -3.56024 & -3.80803 & 1.82662 \\ \mathrm{H} & -2.68480 & -4.32091 & 0.36359 \\ \mathrm{H} & -1.97950 & -3.79784 & -1.83069 \\ \mathrm{H} & -2.52271 & -4.07515 & -3.49231 \\ \mathrm{H} & -1.48942 & -6.23588 & -2.84272 \\ \mathrm{H} & -0.09897 & -5.39337 & -2.13662 \\ \mathrm{H} & 0.79851 & -5.80879 & -4.34832 \\ \mathrm{H} & -0.79363 & -5.69191 & -5.09705 \\ \mathrm{H} & -0.43490 & -3.43575 & -5.52847 \\ \mathrm{H} & 1.10711 & -3.45534 & -4.68012 \\ \mathrm{H} & -0.31920 & 2.79809 & -5.52706 \\ \mathrm{H} & 1.25971 & 2.34529 & -4.86699 \\ \mathrm{H} & 1.26335 & 4.37345 & -3.40696 \\ \mathrm{H} & 0.73978 & 4.98456 & -4.98791 \\ \mathrm{H} & -1.61052 & 4.90083 & -4.33829 \\ \mathrm{H} & -0.87130 & 5.46335 & -2.83361 \\ \mathrm{H} & -1.26476 & 3.34235 & -1.77516 \\ \mathrm{H} & -2.42060 & 3.08606 & -3.07936 \\ \mathrm{H} & -3.15861 & 3.57776 & 1.95496 \\ \mathrm{H} & -2.58621 & 2.97381 & 0.40650 \\ \mathrm{H} & -2.78358 & 5.77877 & 1.30098 \\ \mathrm{H} & 0.08124 & 5.22501 & 0.41791 \\ \mathrm{H} & 0.71043 & 4.37888 & 2.65916 \\ \mathrm{H} & -0.87459 & 4.83950 & 3.31114 \\ \mathrm{H} & 1.44797 & -0.28104 & 5.88902 \\ \mathrm{H} & 2.35820 & -1.52084 & 5.01134 \\ \mathrm{H} & 1.74273 & -2.76092 & 7.00151 \\ \mathrm{H} & 0.15785 & -1.96627 & 7.09056 \\ \mathrm{H} & 1.05790 & -3.97400 & 4.94100 \\ \mathrm{H} & -0.34383 & -4.15076 & 6.01685 \\ \mathrm{H} & -0.78130 & -3.09080 & 3.56075 \\ \mathrm{H} & -1.50636 & -2.28795 & 4.94919 \\ \mathrm{H} & -0.48864 & 6.50058 & 1.51328\end{array}$

- DFT Input File - (dicPDI)Fe(cis-2,3-dimethylmetallacyclopentane)

! UKS B3LYP RIJCOSX def2-SVP def2/J Normalprint SlowConv TightSCF Opt NumFreq Pal8 UCO

\%basis NewGTO 26 "def2-TZVP(-f)" end NewGTO 7 "def2-TZVP(-f)" end NewAuxGTO 26 "def2/J" end NewAuxGTO 7 "def2/J" end end

$\%$ SCF BrokenSym 3,1 Maxiter 5000

TolE $1 \mathrm{e}-7$

TolErr 1e-6

end 


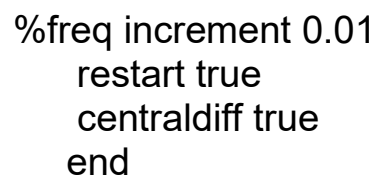

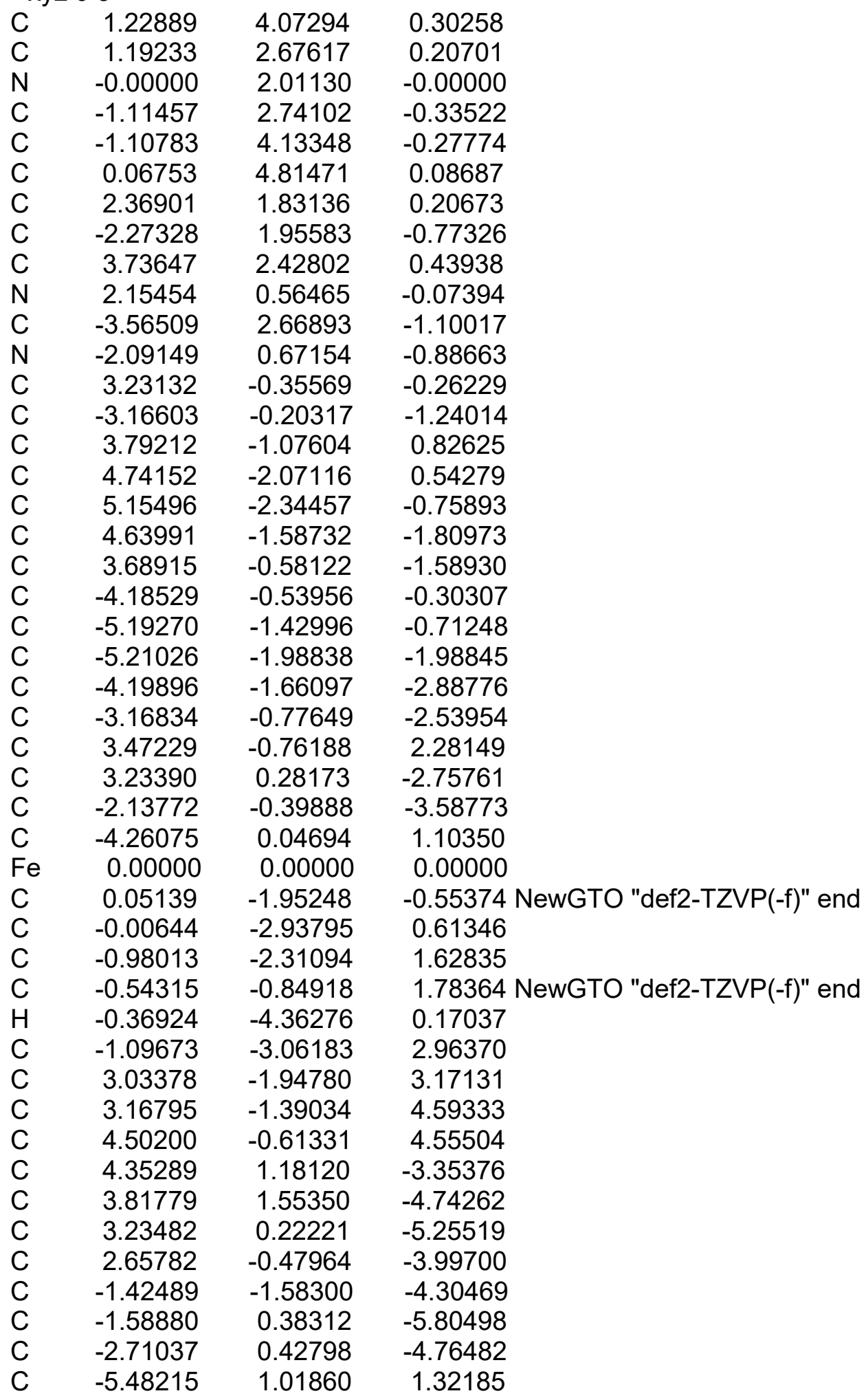




\begin{tabular}{|c|c|c|c|}
\hline C & -6.38236 & 0.34672 & 2.37284 \\
\hline C & -5.38658 & -0.41101 & 3.25596 \\
\hline C & -4.41270 & -1.02033 & 2.23622 \\
\hline C & 4.67670 & -0.15744 & 3.07883 \\
\hline C & -1.07092 & -1.07428 & -5.73369 \\
\hline $\mathrm{H}$ & 2.65162 & -0.02900 & 2.30319 \\
\hline $\mathrm{H}$ & 0.00813 & -1.13788 & -5.94663 \\
\hline $\mathrm{H}$ & 2.17000 & 4.57936 & 0.52218 \\
\hline $\mathrm{H}$ & -2.00454 & 4.69907 & -0.53320 \\
\hline $\mathrm{H}$ & 0.08463 & 5.90500 & 0.14242 \\
\hline $\mathrm{H}$ & 3.82464 & 2.84726 & 1.45559 \\
\hline $\mathrm{H}$ & 3.93478 & 3.25040 & -0.26652 \\
\hline $\mathrm{H}$ & 4.52325 & 1.67556 & 0.30935 \\
\hline $\mathrm{H}$ & -3.99578 & 3.15040 & -0.20831 \\
\hline $\mathrm{H}$ & -4.30772 & 1.97500 & -1.50972 \\
\hline $\mathrm{H}$ & -3.38841 & 3.46274 & -1.84341 \\
\hline $\mathrm{H}$ & 5.17682 & -2.64064 & 1.36831 \\
\hline $\mathrm{H}$ & 5.88835 & -3.13198 & -0.95218 \\
\hline $\mathrm{H}$ & 4.99219 & -1.77527 & -2.82840 \\
\hline $\mathrm{H}$ & -5.99062 & -1.69000 & -0.01200 \\
\hline $\mathrm{H}$ & -6.01049 & -2.67235 & -2.28365 \\
\hline $\mathrm{H}$ & -4.21761 & -2.08890 & -3.89296 \\
\hline $\mathrm{H}$ & 2.44743 & 0.95632 & -2.39474 \\
\hline $\mathrm{H}$ & -1.36324 & 0.21653 & -3.10543 \\
\hline $\mathrm{H}$ & -3.33836 & 0.61177 & 1.28784 \\
\hline $\mathrm{H}$ & -0.85630 & -2.03743 & -1.16261 \\
\hline $\mathrm{H}$ & 0.93651 & -2.07100 & -1.19036 \\
\hline C & 0.99066 & -2.97114 & 1.08964 \\
\hline $\mathrm{H}$ & -1.97577 & -2.31484 & 1.14837 \\
\hline $\mathrm{H}$ & 0.38192 & -0.77204 & 2.36552 \\
\hline $\mathrm{H}$ & -1.29324 & -0.21171 & 2.26193 \\
\hline $\mathrm{H}$ & -1.71660 & -2.50360 & 3.68444 \\
\hline $\mathrm{H}$ & -1.55416 & -4.05853 & 2.84316 \\
\hline $\mathrm{H}$ & -0.10619 & -3.20363 & 3.42831 \\
\hline $\mathrm{H}$ & 2.02386 & -2.30791 & 2.93180 \\
\hline $\mathrm{H}$ & 3.72027 & -2.80134 & 3.03973 \\
\hline $\mathrm{H}$ & 3.14112 & -2.17226 & 5.36937 \\
\hline $\mathrm{H}$ & 2.33043 & -0.70136 & 4.80282 \\
\hline $\mathrm{H}$ & 4.51914 & 0.23053 & 5.26195 \\
\hline $\mathrm{H}$ & 5.33039 & -1.27933 & 4.84799 \\
\hline $\mathrm{H}$ & 5.62518 & -0.53300 & 2.66598 \\
\hline $\mathrm{H}$ & 4.71161 & 0.93822 & 2.98025 \\
\hline $\mathrm{H}$ & 5.28789 & 0.60414 & -3.45507 \\
\hline $\mathrm{H}$ & 4.58350 & 2.05048 & -2.71642 \\
\hline $\mathrm{H}$ & 3.01919 & 2.31038 & -4.64787 \\
\hline $\mathrm{H}$ & 4.58712 & 1.97788 & -5.40815 \\
\hline $\mathrm{H}$ & 4.04758 & -0.38508 & -5.68785 \\
\hline $\mathrm{H}$ & 2.48391 & 0.35648 & -6.05112 \\
\hline $\mathrm{H}$ & 1.55796 & -0.43476 & -3.97982 \\
\hline $\mathrm{H}$ & 2.92383 & -1.54696 & -3.97854 \\
\hline $\mathrm{H}$ & -2.09186 & -2.45732 & -4.35925 \\
\hline $\mathrm{H}$ & -0.54041 & -1.91139 & -3.73871 \\
\hline
\end{tabular}




$\begin{array}{rrrr}\mathrm{H} & -1.57284 & -1.69231 & -6.49424 \\ \mathrm{H} & -0.78937 & 1.08508 & -5.51142 \\ \mathrm{H} & -3.00823 & 1.44840 & -4.47235 \\ \mathrm{H} & -3.61290 & -0.07150 & -5.16069 \\ \mathrm{H} & -6.01578 & 1.24058 & 0.38587 \\ \mathrm{H} & -5.11473 & 1.97973 & 1.71917 \\ \mathrm{H} & -7.00709 & 1.06552 & 2.92771 \\ \mathrm{H} & -7.06790 & -0.36989 & 1.88505 \\ \mathrm{H} & -4.85681 & 0.29990 & 3.91585 \\ \mathrm{H} & -5.85863 & -1.16631 & 3.90493 \\ \mathrm{H} & -3.44527 & -1.29441 & 2.67749 \\ \mathrm{H} & -4.84088 & -1.94967 & 1.82841 \\ \mathrm{H} & -1.92456 & 0.67564 & -6.81349 \\ \mathrm{H} & 1.69600 & -3.40277 & 0.41061 \\ \mathrm{H} & 0.94187 & -3.56491 & 1.97843 \\ \mathrm{H} & 1.29922 & -1.97785 & 1.34078 \\ * & & & \end{array}$

\%plots format cube

$\operatorname{dim} 1100 \operatorname{dim} 2100 \operatorname{dim} 3100$

SpinDens("dicPDIFemetallacycle.cube");

end

\section{- Calculated Thermochemical Properties - (dicPDI)Fe(cis-2,3-dimethylmetallacyclopentane) (Calculated at 298.15 K)}

Final Gibbs free enthalpy $\quad$... -3254.4917787281

\section{n Optimized Coordiantes - ( ${ }^{\text {dicPDI}}$ )Fe(cis-2,3-dimethylmetallacyclopentane)}

$\begin{array}{llcc}\text { C } & 1.23723421248403 & 4.01259346881712 & -0.42815166932327 \\ \text { C } & 1.21841639107687 & 2.61435140452153 & -0.38747214324055 \\ \text { N } & 0.03818511551348 & 1.90337412502442 & -0.52778668900654 \\ \text { C } & -1.13727086212277 & 2.61720241228868 & -0.65086995788202 \\ \text { C } & -1.14962031974342 & 4.01061598206074 & -0.66821029778575 \\ \text { C } & 0.04957362697356 & 4.73102080666620 & -0.56894054857194 \\ \text { C } & 2.42276461057508 & 1.83052687709569 & -0.15493434105010 \\ \text { C } & -2.37201024088920 & 1.83799605706722 & -0.79485474462787 \\ \text { C } & 3.75373972742693 & 2.53525883433808 & -0.00822629477104 \\ \text { N } & 2.26593657534634 & 0.53623329886940 & -0.05553373194929 \\ \text { C } & -3.68681222855575 & 2.56526654072910 & -0.98662581291550 \\ \text { N } & -2.25671354478177 & 0.54701274418493 & -0.75454485574788 \\ \text { C } & 3.36052204791057 & -0.31872398649501 & 0.26889584225997 \\ \text { C } & -3.35873830122229 & -0.31945873933529 & -0.99944653917810 \\ \text { C } & 3.52302849260687 & -0.70656628028860 & 1.63045826258356 \\ \text { C } & 4.56053510311713 & -1.59319604165031 & 1.95083813894354 \\ \text { C } & 5.42195203881620 & -2.09153160655948 & 0.97276699161545 \\ \text { C } & 5.25342906416624 & -1.70010492788655 & -0.35147509291744 \\ \text { C } & 4.23267664224501 & -0.81346313862186 & -0.73633959635958 \\ \text { C } & -4.00119997260292 & -0.94996129563242 & 0.10284068857823 \\ \text { C } & -5.00067176538030 & -1.89625686190274 & -0.16170989690263\end{array}$




\begin{tabular}{|c|c|c|c|}
\hline & -5.37576126902270 & -2.21626827941733 & -1.46749238303183 \\
\hline & -4.75689791478942 & -1.57208398617695 & -2.53415249439270 \\
\hline & 95948 & 539 & \\
\hline & 2.60123220284817 & -0.15685795281904 & 2.71142003253541 \\
\hline & 4.13280781594094 & -0.40223207474788 & -2.1995020222509 \\
\hline & -3.16011748109309 & 0.10046232649659 & -3.54322461051048 \\
\hline & -3.62883783351043 & -0.57902283239902 & 1.5313988883212 \\
\hline & 0.10299481443191 & -0.16289722431293 & -0.48710761 \\
\hline & 003333855700 & -1.67 & \\
\hline & 0672075545 & -3.1( & \\
\hline & 6694145023 & -3.00969065131719 & 0.0052544640297 \\
\hline & 2633548232 & -1.81038901845282 & 0.7164003931740 \\
\hline & 9156361879 & -3.79077282111742 & -2.0154781023988 \\
\hline & -0.73280376051792 & -4.28264820892291 & 0.85992519035907 \\
\hline & 2.487 & -0.97 & \\
\hline & 1.7 & & \\
\hline & 2.43 & & \\
\hline & 60257264 & & -2.74350 \\
\hline & 20017607 & & -4.25861 \\
\hline & 0800432 & -1.05 & -4.553800 \\
\hline & 4.00 & -1.5 & -3.2 \\
\hline & -2.6 & -0.7 & \\
\hline & 7401 & & \\
\hline & 27141 & 585 & -4.29857 \\
\hline & 35786011 & 8298556 & \\
\hline & 15978960 & 3166739 & 3.56 \\
\hline & -3.8 & -0.7 & \\
\hline & -4.0 & & \\
\hline & 2.9 & $1.2 \varepsilon$ & \\
\hline & 31531 & 92 & 549 \\
\hline & 1.5886675 & -0.11 & $2.27 \varepsilon$ \\
\hline & $-1.589602 C$ & 337186 & -6.28 \\
\hline & 3491039 & 784130 & -0.32 \\
\hline & -2.0 & 62 & -0.7 \\
\hline & 0.05 & & -0. \\
\hline & 3.72 & & \\
\hline & 8168759867 & 3.068 & -0.934395 \\
\hline & 71848924820 & 1.82267804621712 & 0.222002 \\
\hline & 1921114833 & 1566558 & -0.16 \\
\hline & -4.52 & 1.85 & -1. \\
\hline & -3.6 & 3.1 & \\
\hline & & & \\
\hline 4 & 6.22051753174372 & -2.78518356816286 & 1.249276 \\
\hline & 5.93280037731083 & -2.09126454771412 & -1.11272318995024 \\
\hline & -5.49785034221900 & -2.39879368236823 & $0.668230030 s$ \\
\hline & 351866715681 & -2.962 & -1.6521034254666 \\
\hline & -5.06 & -1.8 & \\
\hline & 3.24926778710842 & 0.24235667950576 & -2.32 \\
\hline & -2.32795347614621 & 0.73692766969671 & -3.20552539227680 \\
\hline & -2.52865721888178 & -0.53483027833852 & 1.5767553497488 \\
\hline & -0.98096487433810 & -1.44828730164335 & -2.2889395407512 \\
\hline & 0.77207864503289 & -1.44607091775040 & -264366392976115 \\
\hline
\end{tabular}




\begin{tabular}{|c|c|c|c|}
\hline & & & \\
\hline & -1.72428514472564 & -2.74766875378383 & -0.25243148690187 \\
\hline & 7015143826584 & -2.01186115217728 & \\
\hline & 2857608 & | 38423630929 & 072 \\
\hline & 539078168 & 12483717088913 & 39698 \\
\hline & -1.17044381428171 & -5.12851343060839 & 0.3022310162366 \\
\hline & 0.26834710776812 & -4.58669457719127 & 1.2075875410099 \\
\hline & 1.95284180575157 & -1.92680765236690 & \\
\hline & & 96324067 & \\
\hline & & & \\
\hline & & & \\
\hline & 1.73 & 2.19 & \\
\hline & & & \\
\hline & & & \\
\hline & 2.446 & 2.046 & \\
\hline & & -0.1 & \\
\hline & & & \\
\hline & & & \\
\hline & & & \\
\hline & & & \\
\hline & & -1.085 & -5.3 \\
\hline & & -1.8 & \\
\hline & & -2.4 & \\
\hline & & & \\
\hline & & & \\
\hline & & -0.43 & \\
\hline & $-2.7^{\prime}$ & 2.206 & \\
\hline & -4.4 & 1.892 & \\
\hline & & 0.4 & \\
\hline & & & \\
\hline & & & \\
\hline & & 1.460 & 4.0 \\
\hline & -5.327863135931 & 0.884 & 5270 \\
\hline & -2.77074531708 & -0.7 & 4.1 \\
\hline & & -1.1 & \\
\hline & & -2.50 & \\
\hline & -5.16357379314758 & -1.76030293049880 & 547902882024 \\
\hline & -4.08385479009276 & 1.70873839793109 & -6.39365971879038 \\
\hline & & -3.71556480006767 & -2.24731522346319 \\
\hline & & -4.65068532697093 & $-0.7 \varsigma$ \\
\hline & & & \\
\hline
\end{tabular}

- DFT Input File - trans-1,2-dimethylcyclobutane

! RKS B3LYP RIJCOSX def2-SVP def2/J Normalprint SlowConv TightSCF NumFreq Opt Pal8 UCO

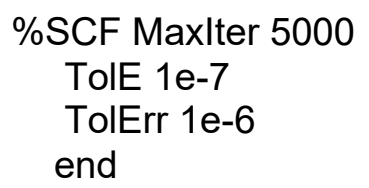

\%freq increment 0.01 
restart true

centraldiff true

end

* xyz 01

$\begin{array}{lrrr}\mathrm{C} & -6.70978 & 1.98569 & 0.14482 \\ \mathrm{C} & -6.85284 & 0.48553 & -0.18480 \\ \mathrm{C} & -5.33136 & 0.47679 & -0.48375 \\ \mathrm{H} & -7.50369 & 0.24770 & -1.03019 \\ \mathrm{H} & -7.15139 & -0.12426 & 0.67608 \\ \mathrm{C} & -5.22233 & 1.70019 & 0.47700 \\ \mathrm{H} & -7.32056 & 2.34690 & 0.97628 \\ \mathrm{H} & -6.86565 & 2.63992 & -0.72108 \\ \mathrm{C} & -4.22717 & 2.77662 & 0.09403 \\ \mathrm{H} & -5.08879 & 1.40546 & 1.52806 \\ \mathrm{H} & -4.35066 & 3.08993 & -0.94779 \\ \mathrm{H} & -3.20187 & 2.41401 & 0.21869 \\ \mathrm{H} & -4.35153 & 3.65914 & 0.72958 \\ \mathrm{C} & -4.57217 & -0.77120 & -0.08142 \\ \mathrm{H} & -5.12030 & 0.73782 & -1.53101 \\ \mathrm{H} & -4.77677 & -1.05242 & 0.95678 \\ \mathrm{H} & -3.49358 & -0.61433 & -0.18284 \\ \mathrm{H} & -4.85120 & -1.61428 & -0.72142 \\ * & & & \\ \end{array}$

end

\section{n Calculated Thermochemical Properties - trans-1,2-dimethylcyclobutane (Calculated at $298.15 \mathrm{~K})$}

Final Gibbs free enthalpy $\ldots-235.502455865792$

- Optimized Coordinates - trans-1,2-diemthylcyclobutane
C -6.74455186035586
C -6.88412208530912
C -5.35024287051463
H $\quad-7.54405645492267$
H $\quad-7.16809470649134$
C -5.23684344319385
H -7.35832390138912
H -6.89056178613011
C -4.20227287893220
H $\quad-5.11131041973908$
H -4.27918711129463
H -3.17843215378777
H -4.32556987598762
C -4.55165772280342
H $\quad-5.14306770908115$
H $\quad-4.71876234498159$
H -3.46849928865213
H $\quad-4.83608338643371$
1.98682833879454
0.48966481051460
0.45256189139298
0.21961355177445
$-0.12204629671421$
1.73125224902944
2.37584548699499
2.64019238397065
2.79224809736501
1.41789611330894
3.11026510746547
2.41204586911079
3.69102660975427
$-0.78950093006334$
0.73521372503498
$-1.08188781061695$
$-0.62468657652406$
$-1.64732262059255$
0.16791647533410
$-0.20825327742434$
$-0.44418134813288$
$-1.04798118052447$
0.66492769501018
0.43540573286242
0.99594965008319
$-0.70891891451981$
0.09789480043858
1.48877486142812
$-0.95646610793105$
0.26078540676693
0.72525735698222
$-0.08571237298080$
$-1.49342734581456$
0.96570361477540
$-0.22261548027915$
$-0.71803956607408$ 
- DFT Input File - ( ${ }^{4}$-dicPDI)Fe

! UKS B3LYP RIJCOSX def2-SVP def2/J Normalprint SlowConv TightSCF Opt NumFreq Pal8 UCO

\%basis NewGTO 26 "def2-TZVP(-f)" end NewGTO 7 "def2-TZVP(-f)" end NewAuxGTO 26 "def2/J" end NewAuxGTO 7 "def2/J" end end

$\%$ SCF BrokenSym 2,2

Maxlter 5000

TolE 1e-7

TolErr 1e-6

end

\%freq increment 0.01

restart true

centraldiff true

end

${ }^{*}$ xyz 03

$\begin{array}{lrrr}\mathrm{C} & -0.79122 & 4.13374 & -0.37685 \\ \mathrm{C} & -0.78662 & 2.76749 & -0.70135 \\ \mathrm{~N} & 0.32213 & 2.00656 & -0.37426 \\ \mathrm{C} & 1.44168 & 2.54458 & 0.22277 \\ \mathrm{C} & 1.46822 & 3.91742 & 0.51211 \\ \mathrm{C} & 0.34153 & 4.70171 & 0.22190 \\ \mathrm{C} & -1.75953 & 1.99608 & -1.39855 \\ \mathrm{C} & 2.41523 & 1.53562 & 0.49304 \\ \mathrm{C} & -3.12080 & 2.51289 & -1.77018 \\ \mathrm{~N} & -1.35004 & 0.71640 & -1.64144 \\ \mathrm{C} & 3.78614 & 1.83316 & 1.02947 \\ \mathrm{~N} & 1.99691 & 0.28359 & 0.17631 \\ \mathrm{C} & -1.95314 & -0.06006 & -2.65791 \\ \mathrm{C} & 2.84123 & -0.81400 & 0.54755 \\ \mathrm{C} & -2.01846 & 0.41193 & -4.00217 \\ \mathrm{C} & -2.69608 & -0.37603 & -4.94463 \\ \mathrm{C} & -3.24067 & -1.61395 & -4.60691 \\ \mathrm{C} & -3.06146 & -2.11913 & -3.31715 \\ \mathrm{C} & -2.39914 & -1.37197 & -2.33611 \\ \mathrm{C} & 2.79340 & -1.31210 & 1.87871 \\ \mathrm{C} & 3.67234 & -2.34697 & 2.23556 \\ \mathrm{C} & 4.55876 & -2.90266 & 1.31393 \\ \mathrm{C} & 4.56634 & -2.43203 & 0.00222 \\ \mathrm{C} & 3.71761 & -1.39149 & -0.40806 \\ \mathrm{C} & -1.24759 & 1.64599 & -4.45312 \\ \mathrm{C} & -2.14065 & -1.91435 & -0.93788 \\ \mathrm{C} & 3.76125 & -0.91718 & -1.85067 \\ \mathrm{C} & 1.83862 & -0.75120 & 2.92626 \\ \mathrm{C} & 0.15142 & 1.31782 & -5.04443 \\ \mathrm{C} & 0.59641 & 2.61368 & -5.76990\end{array}$




\begin{tabular}{|c|c|c|c|}
\hline C & -0.69265 & 3.46977 & -5.93848 \\
\hline C & -1.85667 & 2.53875 & -5.55156 \\
\hline C & -2.62084 & -3.35336 & -0.63253 \\
\hline C & -1.70392 & -3.82383 & 0.50305 \\
\hline C & -0.32310 & -3.33639 & 0.04055 \\
\hline C & -0.62178 & -1.92435 & -0.51692 \\
\hline C & 5.13463 & -0.36796 & -2.34921 \\
\hline C & 5.18982 & -0.67135 & -3.87611 \\
\hline C & 3.83773 & -1.33843 & -4.21880 \\
\hline C & 3.41382 & -1.99644 & -2.90240 \\
\hline C & 0.92091 & -1.81690 & 3.59555 \\
\hline C & 0.64489 & -1.27355 & 5.00607 \\
\hline C & 2.00986 & -0.70223 & 5.41789 \\
\hline C & 2.53738 & -0.02551 & 4.13466 \\
\hline $\mathrm{Fe}$ & 0.24286 & 0.21762 & -0.66792 \\
\hline $\mathrm{H}$ & -1.65855 & 4.75073 & -0.61932 \\
\hline $\mathrm{H}$ & 2.34534 & 4.36507 & 0.98290 \\
\hline $\mathrm{H}$ & 0.34786 & 5.76583 & 0.46947 \\
\hline $\mathrm{H}$ & -3.88015 & 1.72589 & -1.63684 \\
\hline $\mathrm{H}$ & -3.39961 & 3.36006 & -1.12712 \\
\hline $\mathrm{H}$ & -3.19832 & 2.85587 & -2.81673 \\
\hline $\mathrm{H}$ & 3.88302 & 1.61610 & 2.10771 \\
\hline $\mathrm{H}$ & 4.54702 & 1.22396 & 0.51914 \\
\hline $\mathrm{H}$ & 4.04382 & 2.89035 & 0.87142 \\
\hline $\mathrm{H}$ & -2.78573 & -0.02039 & -5.97410 \\
\hline $\mathrm{H}$ & -3.77668 & -2.20066 & -5.35735 \\
\hline $\mathrm{H}$ & -3.45299 & -3.10857 & -3.07586 \\
\hline $\mathrm{H}$ & 3.66452 & -2.72753 & 3.2608 \\
\hline $\mathrm{H}$ & 5.23621 & -3.70560 & 1.61742 \\
\hline $\mathrm{H}$ & 5.25303 & -2.87767 & -0.72232 \\
\hline $\mathrm{H}$ & -1.07035 & 2.29475 & -3.58476 \\
\hline $\mathrm{H}$ & -2.64698 & -1.23610 & -0.23148 \\
\hline $\mathrm{H}$ & 3.01989 & -0.11164 & -1.96418 \\
\hline $\mathrm{H}$ & 1.18699 & -0.02310 & 2.42571 \\
\hline $\mathrm{H}$ & 0.85816 & 0.98968 & -4.26606 \\
\hline $\mathrm{H}$ & 0.05217 & 0.48645 & -5.76305 \\
\hline $\mathrm{H}$ & 1.06375 & 2.38318 & -6.74065 \\
\hline $\mathrm{H}$ & 1.35313 & 3.16087 & -5.18609 \\
\hline $\mathrm{H}$ & -0.66307 & 4.32888 & -5.24874 \\
\hline $\mathrm{H}$ & -0.80242 & 3.88747 & -6.95187 \\
\hline $\mathrm{H}$ & -2.16036 & 1.93080 & -6.42097 \\
\hline $\mathrm{H}$ & -2.75216 & 3.08640 & -5.21507 \\
\hline $\mathrm{H}$ & -3.69211 & -3.39755 & -0.37981 \\
\hline $\mathrm{H}$ & -2.46293 & -4.00819 & -1.50540 \\
\hline $\mathrm{H}$ & -1.74809 & -4.91006 & 0.67942 \\
\hline $\mathrm{H}$ & -1.98334 & -3.32506 & 1.44894 \\
\hline $\mathrm{H}$ & 0.44531 & -3.33125 & 0.82818 \\
\hline $\mathrm{H}$ & 0.04650 & -3.98697 & -0.76957 \\
\hline $\mathrm{H}$ & 0.06174 & -1.69062 & -1.37076 \\
\hline $\mathrm{H}$ & -0.46751 & -1.20731 & 0.33352 \\
\hline $\mathrm{H}$ & 5.23238 & 0.70692 & -2.12905 \\
\hline $\mathrm{H}$ & 5.96249 & -0.86859 & -1.82399 \\
\hline
\end{tabular}




$\begin{array}{rrrr}\mathrm{H} & 6.01722 & -1.36520 & -4.09725 \\ \mathrm{H} & 5.37289 & 0.23200 & -4.47842 \\ \mathrm{H} & 3.09628 & -0.57071 & -4.49674 \\ \mathrm{H} & 3.90241 & -2.04490 & -5.06190 \\ \mathrm{H} & 4.01083 & -2.90862 & -2.72197 \\ \mathrm{H} & 2.35261 & -2.29411 & -2.87940 \\ \mathrm{H} & 0.00469 & -2.00462 & 3.01244 \\ \mathrm{H} & 1.44777 & -2.78238 & 3.67459 \\ \mathrm{H} & 0.26146 & -2.04164 & 5.69777 \\ \mathrm{H} & -0.10769 & -0.46496 & 4.96747 \\ \mathrm{H} & 1.95908 & -0.01298 & 6.27644 \\ \mathrm{H} & 2.67679 & -1.53151 & 5.71282 \\ \mathrm{H} & 3.63493 & -0.07366 & 4.06639 \\ \mathrm{H} & 2.26169 & 1.04181 & 4.12561 \\ * & & & \end{array}$

\%plots format cube

$\operatorname{dim} 1100 \operatorname{dim} 2100 \operatorname{dim} 3100$

SpinDens("dicPDIFe_BS22_trip.cube");

end

\section{- Calculated Thermochemical Properties - ( $\kappa^{4}$-dicPDI)Fe (Calculated at 298.15 K)}

Final Gibbs free enthalpy $\quad$... -3018.8868965773

- Optimized Coordinates - ( $\kappa^{4}$ _dicPDI)Fe

$\begin{array}{llll}\text { C } & -0.70483920849972 & 4.19487594355798 & -0.46145497856263 \\ \text { C } & -0.69564421763036 & 2.81384251851900 & -0.77394271976701 \\ \text { N } & 0.37189926161007 & 2.05708537123365 & -0.36911839888392 \\ \text { C } & 1.45082299720072 & 2.55879718856921 & 0.30576903875494 \\ \text { C } & 1.48143618290916 & 3.94340528196642 & 0.60518577906933 \\ \text { C } & 0.39309051988181 & 4.73526434526830 & 0.22131746176325 \\ \text { C } & -1.64766926830097 & 2.04568164988213 & -1.50546785071230 \\ \text { C } & 2.39367217842235 & 1.53799811505519 & 0.62345747295232 \\ \text { C } & -2.96044550146283 & 2.63614883759654 & -1.94167021514773 \\ \text { N } & -1.28924365123744 & 0.73372872623768 & -1.68776925214300 \\ \text { C } & 3.72015431823933 & 1.86379700641883 & 1.25110308706940 \\ \text { N } & 1.99989041616595 & 0.27902709130169 & 0.26022301179757 \\ \text { C } & -1.89833717471225 & -0.06114424125216 & -2.68671118163400 \\ \text { C } & 2.83372259362853 & -0.83045467022357 & 0.60990773990536 \\ \text { C } & -1.99846397843695 & 0.38042280716961 & -4.03999836287088 \\ \text { C } & -2.70406254235018 & -0.42118874929090 & -4.95086093425574 \\ \text { C } & -3.24907940187861 & -1.64753710613476 & -4.57995447417533 \\ \text { C } & -3.04148586946652 & -2.12623309405046 & -3.28434217904747 \\ \text { C } & -2.35157979296952 & -1.36618902568880 & -2.33364567007625 \\ \text { C } & 2.78168376139627 & -1.36748604747280 & 1.92616010068540 \\ \text { C } & 3.65598513919178 & -2.41460101450746 & 2.25801714562942 \\ \text { C } & 4.54028009376615 & -2.95121740483364 & 1.32304393196576 \\ \text { C } & 4.54971087769202 & -2.44764160842916 & 0.02389783408111 \\ \text { C } & 3.70726033081758 & -1.39188007186858 & -0.35971817135511\end{array}$




\begin{tabular}{|c|c|c|c|}
\hline & -1.25246097343268 & .60312636285573 & \\
\hline & -2.08370067491067 & .89076396911744 & \\
\hline & .75597555743183 & .88665126141831 & 20044 \\
\hline & 1.83191625574674 & -0.82461797068287 & 2.98654239920241 \\
\hline & 0.01318154549183 & 1.24825180771827 & -5.37991279849591 \\
\hline & 0.40932814935304 & 2.56556484177742 & -6.09061419616946 \\
\hline & -0.86309926531276 & 3.46307983469786 & -6.05474442804930 \\
\hline & 994606081517 & & \\
\hline & 9764 & 0924 & \\
\hline & 659567294 & -3.78782467527568 & \\
\hline & 260760335849 & $-3.328174808 c$ & 0.04107378439615 \\
\hline & 123678341421 & -1.91900651037360 & -0.52988178909583 \\
\hline & 384449729828 & -0.34028997830710 & \\
\hline & 28819233426 & & \\
\hline & & -1.2 & \\
\hline & & & \\
\hline & 0.9 & -1.9 & 60 \\
\hline & & $-1.3 \xi$ & \\
\hline & 55231703990 & -0.80670 & 5.4 \\
\hline & 2.54 & -0.10 & 4.1 \\
\hline & 0.2 & & \\
\hline & -1.5 & & \\
\hline & 2.32 & 4.3 & \\
\hline & & & \\
\hline & & 670 & -2 \\
\hline & & 3.0729 & \\
\hline & -2.8 & 360 & \\
\hline & & & \\
\hline & & & \\
\hline & 4.22 & & \\
\hline & & & -5 . \\
\hline & -3.80 & -2.2 & -5. \\
\hline & -3.4 & -3.1( & -3. \\
\hline & & -2.8 & \\
\hline & & & \\
\hline & & -2.8 & -0.7 \\
\hline & & & -3. \\
\hline & -2.57168004543495 & -1.19 & 139 \\
\hline & 3.022 & -0.07 & -1.8 \\
\hline & 1.170 & -0.0 & \\
\hline & & & -4.7 \\
\hline & -0.24 & & \\
\hline & 0.755 & & 257 \\
\hline & 1.24423074062011 & 3.059867439 & 7452661 \\
\hline & -0.70292926046855 & 4.310057439 & -5.3 \\
\hline & -1.1096095770 & 3790 & \\
\hline & -2.45 & 2.00 & \\
\hline & -2.79366350728 & & \\
\hline & -3.64776549114741 & -3.34429967593330 & -0.32778467004038 \\
\hline & -2.44469972942449 & -3.98856460807522 & -1.46249824159610 \\
\hline & -1.71071734741268 & -4.87101662519051 & 0.72149657637249 \\
\hline & -1.91004975191466 & -3.27324770551802 & 1.47450684749702 \\
\hline
\end{tabular}




\begin{tabular}{|c|c|c|c|}
\hline $\mathrm{H}$ & 0.50790756110919 & -3.32313361880294 & 0.8167 \\
\hline- & 0.07491933814983 & 99390295133879 & 3966 \\
\hline $\mathrm{H}$ & 0.11409360046740 & -1.70291932960887 & -1.39236716809818 \\
\hline $\mathrm{H}$ & -0.38944283285685 & -1.20286416657904 & 0.31581313946514 \\
\hline & 5.24273504878404 & 0.72769407567494 & -2.0315544648695 \\
\hline I & 5.95689923432850 & -0.86164499402468 & -1.76673693515378 \\
\hline & 6.00917886401223 & -1.30253057552460 & -4.04982981092919 \\
\hline & 5.37162009395490 & 0.30537796426864 & -4.39502486116660 \\
\hline & 3.09351287622898 & -0.48709024376965 & -4.4371894708102 \\
\hline $\mathrm{H}$ & 3.89221912147957 & -1.95592172629343 & -5.02396725032560 \\
\hline $\mathrm{H}$ & 3.98719577596532 & -2.86266820945787 & -2.70222702253132 \\
\hline $\mathrm{H}$ & 2.33524689051722 & -2.23118187755631 & -2.85005645429230 \\
\hline$\exists$ & 0.00280360415461 & -2.08445435487794 & 3.09448405095164 \\
\hline & 1.46099598183661 & -2.86759281076222 & 3.7138 \\
\hline 7 & 0.31964061009247 & -2.16011105421652 & 5.773410922 \\
\hline $\mathrm{H}$ & -0.07318513233828 & -0.57504173588723 & 946600411 \\
\hline $\mathrm{H}$ & 2.02092169062100 & -0.13418917295457 & 6.3454863645309 \\
\hline $\mathrm{H}$ & 2.73505333393921 & -1.63622568758093 & 5.7345806503338 \\
\hline & 3.63739988979649 & -0.12331748096853 & 4.0956509188279 \\
\hline & 2.24112368634569 & 0.959082681316 & 4.2031995206525 \\
\hline
\end{tabular}

\title{
- DFT Input File - ( $\kappa^{4}$-dicPDI)Fe (no agostic)
}

! UKS B3LYP RIJCOSX def2-SVP def2/J Normalprint SlowConv TightSCF NumFreq Pal8 UCO

\author{
end \\ \%SCF BrokenSym 2,2 \\ Maxlter 5000 \\ TolE 1e-7 \\ TolErr 1e-6 \\ end
}

\%basis NewGTO 26 "def2-TZVP(-f)" end NewGTO 7 "def2-TZVP(-f)" end NewAuxGTO 26 "def2/J" end NewAuxGTO 7 "def2/J" end

$\begin{array}{lrrr}{ }^{*} \text { xyz } 03 & 3 & & \\ \mathrm{C} & 3.77809 & 1.39968 & -0.64787 \\ \mathrm{C} & 2.38226 & 1.36432 & -0.49210 \\ \mathrm{~N} & 1.74498 & 0.15746 & -0.44999 \\ \mathrm{C} & 2.40217 & -1.03473 & -0.56157 \\ \mathrm{C} & 3.79852 & -1.03669 & -0.72125 \\ \mathrm{C} & 4.47630 & 0.18923 & -0.75806 \\ \mathrm{C} & 1.49945 & -2.15122 & -0.47203 \\ \mathrm{C} & 1.45812 & 2.45874 & -0.36010 \\ \mathrm{C} & 1.92993 & 3.88516 & -0.38461\end{array}$




\begin{tabular}{|c|c|c|c|}
\hline $\mathrm{N}$ & 0.17081 & 2.07269 & -0.22567 \\
\hline C & 1.99783 & -3.56499 & -0.57182 \\
\hline$N$ & 0.20798 & -1.79702 & -0.30172 \\
\hline C & -0.85618 & 3.06125 & -0.05736 \\
\hline C & -0.79024 & -2.80131 & -0.06116 \\
\hline C & -1.55546 & 3.54850 & -1.19130 \\
\hline C & -2.60867 & 4.45350 & -0.98513 \\
\hline C & -2.96748 & 4.87464 & 0.29400 \\
\hline C & -2.26060 & 4.39807 & 1.39666 \\
\hline C & -1.19655 & 3.49602 & 1.24916 \\
\hline C & -1.01166 & -3.20639 & 1.28552 \\
\hline C & -2.03419 & -4.12463 & 1.55951 \\
\hline C & -2.83370 & -4.64035 & 0.54190 \\
\hline C & -2.60331 & -4.23961 & -0.77026 \\
\hline C & -1.58502 & -3.32832 & -1.11178 \\
\hline C & -1.18838 & 3.13487 & -2.60678 \\
\hline C & -0.44906 & 3.01172 & 2.47977 \\
\hline $\mathrm{H}$ & -3.79466 & 5.57567 & 0.43432 \\
\hline C & -1.42427 & -3.08407 & -2.61117 \\
\hline C & -0.13656 & -2.70995 & 2.42422 \\
\hline C & -2.29202 & 2.42104 & -3.42085 \\
\hline C & -1.71073 & 2.40490 & -4.84151 \\
\hline $\mathrm{H}$ & -0.33918 & 2.43733 & -2.54804 \\
\hline C & -1.29643 & 2.23380 & 3.50956 \\
\hline C & -0.35421 & 2.12929 & 4.71477 \\
\hline C & 0.33455 & 3.51587 & 4.78110 \\
\hline C & 0.21227 & 4.11816 & 3.35126 \\
\hline C & 0.79891 & -3.77667 & 3.04061 \\
\hline C & 1.34861 & -3.07062 & 4.28603 \\
\hline C & 0.13961 & -2.27556 & 4.84656 \\
\hline C & -0.86985 & -2.14613 & 3.66880 \\
\hline C & -0.64944 & -4.21521 & -3.34385 \\
\hline C & -0.32535 & -3.65116 & -4.75381 \\
\hline C & -0.69949 & -2.14508 & -4.69935 \\
\hline$C$ & -0.78553 & -1.81218 & -3.20005 \\
\hline $\mathrm{Fe}$ & -0.12954 & 0.13502 & -0.24314 \\
\hline $\mathrm{H}$ & 4.30678 & 2.35347 & -0.68487 \\
\hline $\mathrm{H}$ & 4.34283 & -1.97737 & -0.81679 \\
\hline $\mathrm{H}$ & 5.56205 & 0.20236 & -0.88344 \\
\hline $\mathrm{H}$ & 2.36932 & 4.14593 & -1.36464 \\
\hline $\mathrm{H}$ & 1.11318 & 4.58945 & -0.18477 \\
\hline $\mathrm{H}$ & 2.71833 & 4.05740 & 0.36798 \\
\hline $\mathrm{H}$ & 2.41970 & -3.76958 & -1.57273 \\
\hline $\mathrm{H}$ & 2.80708 & -3.75839 & 0.15269 \\
\hline $\mathrm{H}$ & 1.19758 & -4.29257 & -0.38843 \\
\hline $\mathrm{H}$ & -3.16001 & 4.83585 & -1.84789 \\
\hline $\mathrm{H}$ & -2.54028 & 4.73726 & 2.39818 \\
\hline $\mathrm{H}$ & -2.20864 & -4.44443 & 2.59039 \\
\hline $\mathrm{H}$ & -3.23061 & -4.64217 & -1.57051 \\
\hline $\mathrm{H}$ & 0.34919 & 2.33013 & 2.14941 \\
\hline $\mathrm{H}$ & -2.45238 & -3.11083 & -3.01795 \\
\hline $\mathrm{H}$ & 0.51185 & -1.90971 & 2.03871 \\
\hline
\end{tabular}




\begin{tabular}{rrrr}
$\mathrm{H}$ & -3.63277 & -5.34961 & 0.77381 \\
$\mathrm{H}$ & -2.53557 & 1.41998 & -3.02984 \\
$\mathrm{H}$ & -3.22397 & 3.01215 & -3.39391 \\
$\mathrm{C}$ & -1.03101 & 3.79039 & -4.99693 \\
$\mathrm{C}$ & -0.77382 & 4.29996 & -3.54994 \\
$\mathrm{H}$ & -1.39336 & 5.18615 & -3.34370 \\
$\mathrm{H}$ & -2.19572 & 2.81565 & 3.77867 \\
$\mathrm{H}$ & -1.64560 & 1.26188 & 3.12587 \\
$\mathrm{H}$ & -0.42402 & 5.01579 & 3.36750 \\
$\mathrm{H}$ & 1.18243 & 4.43550 & 2.93791 \\
$\mathrm{H}$ & 0.21048 & -4.66632 & 3.32805 \\
$\mathrm{H}$ & 1.58372 & -4.11562 & 2.34666 \\
$\mathrm{H}$ & -1.77540 & -2.73426 & 3.87990 \\
$\mathrm{H}$ & -1.20930 & -1.11185 & 3.50220 \\
$\mathrm{H}$ & 0.27721 & -4.44138 & -2.79325 \\
$\mathrm{H}$ & -1.23125 & -5.14978 & -3.37610 \\
$\mathrm{H}$ & 0.22480 & -1.65392 & -2.79666 \\
$\mathrm{H}$ & -1.37194 & -0.90315 & -2.99551 \\
$\mathrm{H}$ & 0.27042 & 4.60743 & -3.38008 \\
$\mathrm{H}$ & 2.14901 & -2.37536 & 3.98046 \\
$\mathrm{H}$ & 1.78837 & -3.76384 & 5.02117 \\
$\mathrm{H}$ & -0.32227 & -2.81273 & 5.68987 \\
$\mathrm{H}$ & 0.45040 & -1.29429 & 5.23840 \\
$\mathrm{H}$ & -0.88883 & -4.17491 & -5.54084 \\
$\mathrm{H}$ & 0.74106 & -3.79071 & -4.99397 \\
$\mathrm{H}$ & 0.01964 & -1.50612 & -5.23453 \\
$\mathrm{H}$ & -1.68254 & -1.98211 & -5.17321 \\
$\mathrm{H}$ & -2.46798 & 2.20593 & -5.61654 \\
$\mathrm{H}$ & -0.95653 & 1.60327 & -4.92111 \\
$\mathrm{H}$ & -0.10292 & 3.72597 & -5.58592 \\
$\mathrm{H}$ & -1.69214 & 4.48684 & -5.53804 \\
$\mathrm{H}$ & -0.17301 & 4.16423 & 5.51274 \\
$\mathrm{H}$ & 1.38189 & 3.43821 & 5.11332 \\
$\mathrm{H}$ & 0.39935 & 1.34590 & 4.52234 \\
$*$ & -0.87067 & 1.85415 & 5.64854 \\
& & & \\
\hline & & & \\
\hline
\end{tabular}

$\%$ plots format cube

$\operatorname{dim} 1100 \operatorname{dim} 2100 \operatorname{dim} 3100$

SpinDens("dicPDIFenoagos_BS22_trip.cube");

end

- Calculated Thermochemical Properties - ( $K^{4}$-dicPDI)Fe (no agostic) (Calculated at 298.15 K)

Final Gibbs free enthalpy $\quad$... -3018.903071644

- Optimized Coordinates - ( $\kappa^{4}$-dicPDI)Fe (no agostic)

$\begin{array}{llll}\mathrm{C} & 3.77809 & 1.39968 & -0.64787 \\ \mathrm{C} & 2.38226 & 1.36432 & -0.49210 \\ \mathrm{~N} & 1.74498 & 0.15746 & -0.44999\end{array}$




\begin{tabular}{|c|c|c|c|}
\hline C & 2.40217 & -1.03473 & -0.56157 \\
\hline C & 3.79852 & -1.03669 & -0.72125 \\
\hline C & 4.47630 & 0.18923 & -0.75806 \\
\hline C & 1.49945 & -2.15122 & -0.47203 \\
\hline C & 1.45812 & 2.45874 & -0.36010 \\
\hline C & 1.92993 & 3.88516 & -0.38461 \\
\hline $\mathrm{N}$ & 0.17081 & 2.07269 & -0.22567 \\
\hline C & 1.99783 & -3.56499 & -0.57182 \\
\hline $\mathrm{N}$ & 0.20798 & -1.79702 & -0.30172 \\
\hline C & -0.85618 & 3.06125 & -0.05736 \\
\hline C & -0.79024 & -2.80131 & -0.06116 \\
\hline C & -1.55546 & 3.54850 & -1.19130 \\
\hline C & -2.60867 & 4.45350 & -0.98513 \\
\hline C & -2.96748 & 4.87464 & 0.29400 \\
\hline C & -2.26060 & 4.39807 & 1.39666 \\
\hline C & -1.19655 & 3.49602 & 1.24916 \\
\hline C & -1.01166 & -3.20639 & 1.28552 \\
\hline C & -2.03419 & -4.12463 & 1.55951 \\
\hline C & -2.83370 & -4.64035 & 0.54190 \\
\hline C & -2.60331 & -4.23961 & -0.77026 \\
\hline C & -1.58502 & -3.32832 & -1.11178 \\
\hline C & -1.18838 & 3.13487 & -2.60678 \\
\hline C & -0.44906 & 3.01172 & 2.47977 \\
\hline $\mathrm{H}$ & -3.79466 & 5.57567 & 0.43432 \\
\hline C & -1.42427 & -3.08407 & -2.61117 \\
\hline C & -0.13656 & -2.70995 & 2.42422 \\
\hline C & -2.29202 & 2.42104 & -3.42085 \\
\hline C & -1.71073 & 2.40490 & -4.84151 \\
\hline $\mathrm{H}$ & -0.33918 & 2.43733 & -2.54804 \\
\hline C & -1.29643 & 2.23380 & 3.50956 \\
\hline C & -0.35421 & 2.12929 & 4.71477 \\
\hline C & 0.33455 & 3.51587 & 4.78110 \\
\hline C & 0.21227 & 4.11816 & 3.35126 \\
\hline C & 0.79891 & -3.77667 & 3.04061 \\
\hline C & 1.34861 & -3.07062 & 4.28603 \\
\hline C & 0.13961 & -2.27556 & 4.84656 \\
\hline C & -0.86985 & -2.14613 & 3.66880 \\
\hline C & -0.64944 & -4.21521 & -3.34385 \\
\hline C & -0.32535 & -3.65116 & -4.75381 \\
\hline C & -0.69949 & -2.14508 & -4.69935 \\
\hline C & -0.78553 & -1.81218 & -3.20005 \\
\hline $\mathrm{Fe}$ & -0.12954 & 0.13502 & -0.24314 \\
\hline $\mathrm{H}$ & 4.30678 & 2.35347 & -0.68487 \\
\hline $\mathrm{H}$ & 4.34283 & -1.97737 & -0.81679 \\
\hline $\mathrm{H}$ & 5.56205 & 0.20236 & -0.88344 \\
\hline $\mathrm{H}$ & 2.36932 & 4.14593 & -1.36464 \\
\hline $\mathrm{H}$ & 1.11318 & 4.58945 & -0.18477 \\
\hline $\mathrm{H}$ & 2.71833 & 4.05740 & 0.36798 \\
\hline $\mathrm{H}$ & 2.41970 & -3.76958 & -1.57273 \\
\hline $\mathrm{H}$ & 2.80708 & -3.75839 & 0.15269 \\
\hline $\mathrm{H}$ & 1.19758 & -4.29257 & -0.38843 \\
\hline $\mathrm{H}$ & -3.16001 & 4.83585 & -1.84789 \\
\hline
\end{tabular}




\begin{tabular}{rrrr}
$\mathrm{H}$ & -2.54028 & 4.73726 & 2.39818 \\
$\mathrm{H}$ & -2.20864 & -4.44443 & 2.59039 \\
$\mathrm{H}$ & -3.23061 & -4.64217 & -1.57051 \\
$\mathrm{H}$ & 0.34919 & 2.33013 & 2.14941 \\
$\mathrm{H}$ & -2.45238 & -3.11083 & -3.01795 \\
$\mathrm{H}$ & 0.51185 & -1.90971 & 2.03871 \\
$\mathrm{H}$ & -3.63277 & -5.34961 & 0.77381 \\
$\mathrm{H}$ & -2.53557 & 1.41998 & -3.02984 \\
$\mathrm{H}$ & -3.22397 & 3.01215 & -3.39391 \\
$\mathrm{C}$ & -1.03101 & 3.79039 & -4.99693 \\
$\mathrm{C}$ & -0.77382 & 4.29996 & -3.54994 \\
$\mathrm{H}$ & -1.39336 & 5.18615 & -3.34370 \\
$\mathrm{H}$ & -2.19572 & 2.81565 & 3.77867 \\
$\mathrm{H}$ & -1.64560 & 1.26188 & 3.12587 \\
$\mathrm{H}$ & -0.42402 & 5.01579 & 3.36750 \\
$\mathrm{H}$ & 1.18243 & 4.43550 & 2.93791 \\
$\mathrm{H}$ & 0.21048 & -4.66632 & 3.32805 \\
$\mathrm{H}$ & 1.58372 & -4.11562 & 2.34666 \\
$\mathrm{H}$ & -1.77540 & -2.73426 & 3.87990 \\
$\mathrm{H}$ & -1.20930 & -1.11185 & 3.50220 \\
$\mathrm{H}$ & 0.27721 & -4.44138 & -2.79325 \\
$\mathrm{H}$ & -1.23125 & -5.14978 & -3.37610 \\
$\mathrm{H}$ & 0.22480 & -1.65392 & -2.79666 \\
$\mathrm{H}$ & -1.37194 & -0.90315 & -2.99551 \\
$\mathrm{H}$ & 0.27042 & 4.60743 & -3.38008 \\
$\mathrm{H}$ & 2.14901 & -2.37536 & 3.98046 \\
$\mathrm{H}$ & 1.78837 & -3.76384 & 5.02117 \\
$\mathrm{H}$ & -0.32227 & -2.81273 & 5.68987 \\
$\mathrm{H}$ & 0.45040 & -1.29429 & 5.23840 \\
$\mathrm{H}$ & -0.88883 & -4.17491 & -5.54084 \\
$\mathrm{H}$ & 0.74106 & -3.79071 & -4.99397 \\
$\mathrm{H}$ & 0.01964 & -1.50612 & -5.23453 \\
$\mathrm{H}$ & -1.68254 & -1.98211 & -5.17321 \\
$\mathrm{H}$ & -2.46798 & 2.20593 & -5.61654 \\
$\mathrm{H}$ & -0.95653 & 1.60327 & -4.92111 \\
$\mathrm{H}$ & -0.10292 & 3.72597 & -5.58592 \\
$\mathrm{H}$ & -1.69214 & 4.48684 & -5.53804 \\
& -0.17301 & 4.16423 & 5.51274 \\
& 1.38189 & 3.43821 & 5.11332 \\
\hline & -0.87067 & 1.85415 & 5.64854
\end{tabular}

- DFT Input File - ( ${ }^{\text {dic } P D I) F e}\left(\mathbf{N}_{2}\right)$

! UKS B3LYP RIJCOSX def2-SVP def2/J Normalprint SlowConv TightSCF NumFreq Pal8 UCO

\%basis NewGTO 26 "def2-TZVP(-f)" end

NewGTO 7 "def2-TZVP(-f)" end

NewAuxGTO 26 "def2/J" end

NewAuxGTO 7 "def2/J" end

end 


\begin{tabular}{|c|c|c|c|}
\hline & $\begin{array}{l}\text { BrokenSy } \\
\text { slter } 5000 \\
=1 \mathrm{e}-7 \\
\text { Err } 1 \mathrm{e}-6\end{array}$ & 2,2 & \\
\hline \multicolumn{4}{|c|}{$\begin{array}{l}\text { \%freq increment } 0.01 \\
\text { restart true } \\
\text { centraldiff true } \\
\text { end }\end{array}$} \\
\hline \multicolumn{4}{|c|}{ * xyz 03} \\
\hline C & 3.77809 & 1.39968 & -0.64787 \\
\hline C & 2.38226 & 1.36432 & -0.49210 \\
\hline$N$ & 1.74498 & 0.15746 & -0.44999 \\
\hline C & 2.40217 & -1.03473 & -0.56157 \\
\hline C & 3.79852 & -1.03669 & -0.72125 \\
\hline C & 4.47630 & 0.18923 & -0.75806 \\
\hline C & 1.49945 & -2.15122 & -0.47203 \\
\hline C & 1.45812 & 2.45874 & -0.36010 \\
\hline C & 1.92993 & 3.88516 & -0.38461 \\
\hline$N$ & 0.17081 & 2.07269 & -0.22567 \\
\hline C & 1.99783 & -3.56499 & -0.57182 \\
\hline $\mathrm{N}$ & 0.20798 & -1.79702 & -0.30172 \\
\hline C & -0.85618 & 3.06125 & -0.05736 \\
\hline C & -0.79024 & -2.80131 & -0.06116 \\
\hline C & -1.55546 & 3.54850 & -1.19130 \\
\hline C & -2.60867 & 4.45350 & -0.98513 \\
\hline C & -2.96748 & 4.87464 & 0.29400 \\
\hline C & -2.26060 & 4.39807 & 1.39666 \\
\hline C & -1.19655 & 3.49602 & 1.24916 \\
\hline C & -1.01166 & -3.20639 & 1.28552 \\
\hline C & -2.03419 & -4.12463 & 1.55951 \\
\hline C & -2.83370 & -4.64035 & 0.54190 \\
\hline C & -2.60331 & -4.23961 & -0.77026 \\
\hline C & -1.58502 & -3.32832 & -1.11178 \\
\hline C & -1.18838 & 3.13487 & -2.60678 \\
\hline C & -0.44906 & 3.01172 & 2.47977 \\
\hline $\mathrm{H}$ & -3.79466 & 5.57567 & 0.43432 \\
\hline C & -1.42427 & -3.08407 & -2.61117 \\
\hline C & -0.13656 & -2.70995 & 2.42422 \\
\hline C & -2.29202 & 2.42104 & -3.42085 \\
\hline C & -1.71073 & 2.40490 & -4.84151 \\
\hline $\mathrm{H}$ & -0.33918 & 2.43733 & -2.54804 \\
\hline C & -1.29643 & 2.23380 & 3.50956 \\
\hline C & -0.35421 & 2.12929 & 4.71477 \\
\hline C & 0.33455 & 3.51587 & 4.78110 \\
\hline C & 0.21227 & 4.11816 & 3.35126 \\
\hline C & 0.79891 & -3.77667 & 3.04061 \\
\hline C & 1.34861 & -3.07062 & 4.28603 \\
\hline C & 0.13961 & -2.27556 & 4.84656 \\
\hline C & -0.86985 & -2.14613 & 3.66880 \\
\hline
\end{tabular}




\begin{tabular}{|c|c|c|c|}
\hline C & -0.64944 & -4.21521 & -3.34385 \\
\hline C & -0.32535 & -3.65116 & -4.75381 \\
\hline C & -0.69949 & -2.14508 & -4.69935 \\
\hline $\mathrm{C}$ & -0.78553 & -1.81218 & -3.20005 \\
\hline $\mathrm{Fe}$ & -0.12954 & 0.13502 & -0.24314 \\
\hline$N$ & -1.96172 & 0.11606 & -0.04636 \\
\hline $\mathrm{H}$ & 4.30678 & 2.35347 & -0.68487 \\
\hline $\mathrm{H}$ & 4.34283 & -1.97737 & -0.81679 \\
\hline $\mathrm{H}$ & 5.56205 & 0.20236 & -0.88344 \\
\hline $\mathrm{H}$ & 2.36932 & 4.14593 & -1.36464 \\
\hline $\mathrm{H}$ & 1.11318 & 4.58945 & -0.18477 \\
\hline $\mathrm{H}$ & 2.71833 & 4.05740 & 0.36798 \\
\hline $\mathrm{H}$ & 2.41970 & -3.76958 & -1.57273 \\
\hline $\mathrm{H}$ & 2.80708 & -3.75839 & 0.15269 \\
\hline $\mathrm{H}$ & 1.19758 & -4.29257 & -0.38843 \\
\hline $\mathrm{H}$ & -3.16001 & 4.83585 & -1.84789 \\
\hline $\mathrm{H}$ & -2.54028 & 4.73726 & 2.39818 \\
\hline $\mathrm{H}$ & -2.20864 & -4.44443 & 2.59039 \\
\hline $\mathrm{H}$ & -3.23061 & -4.64217 & -1.57051 \\
\hline $\mathrm{H}$ & 0.34919 & 2.33013 & 2.14941 \\
\hline $\mathrm{H}$ & -2.45238 & -3.11083 & -3.01795 \\
\hline $\mathrm{H}$ & 0.51185 & -1.90971 & 2.03871 \\
\hline $\mathrm{H}$ & -3.63277 & -5.34961 & 0.77381 \\
\hline $\mathrm{H}$ & -2.53557 & 1.41998 & -3.02984 \\
\hline $\mathrm{H}$ & -3.22397 & 3.01215 & -3.39391 \\
\hline C & -1.03101 & 3.79039 & -4.99693 \\
\hline C & -0.77382 & 4.29996 & -3.54994 \\
\hline $\mathrm{H}$ & -1.39336 & 5.18615 & -3.34370 \\
\hline $\mathrm{H}$ & -2.19572 & 2.81565 & 3.77867 \\
\hline $\mathrm{H}$ & -1.64560 & 1.26188 & 3.12587 \\
\hline $\mathrm{H}$ & -0.42402 & 5.01579 & 3.36750 \\
\hline $\mathrm{H}$ & 1.18243 & 4.43550 & 2.93791 \\
\hline $\mathrm{H}$ & 0.21048 & -4.66632 & 3.32805 \\
\hline $\mathrm{H}$ & 1.58372 & -4.11562 & 2.34666 \\
\hline $\mathrm{H}$ & -1.77540 & -2.73426 & 3.87990 \\
\hline $\mathrm{H}$ & -1.20930 & -1.11185 & 3.50220 \\
\hline $\mathrm{H}$ & 0.27721 & -4.44138 & -2.79325 \\
\hline $\mathrm{H}$ & -1.23125 & -5.14978 & -3.37610 \\
\hline $\mathrm{H}$ & 0.22480 & -1.65392 & -2.79666 \\
\hline $\mathrm{H}$ & -1.37194 & -0.90315 & -2.99551 \\
\hline $\mathrm{H}$ & 0.27042 & 4.60743 & -3.38008 \\
\hline $\mathrm{N}$ & -3.06556 & 0.10632 & 0.06292 \\
\hline $\mathrm{H}$ & 2.14901 & -2.37536 & 3.98046 \\
\hline $\mathrm{H}$ & 1.78837 & -3.76384 & 5.02117 \\
\hline $\mathrm{H}$ & -0.32227 & -2.81273 & 5.68987 \\
\hline $\mathrm{H}$ & 0.45040 & -1.29429 & 5.23840 \\
\hline $\mathrm{H}$ & -0.88883 & -4.17491 & -5.54084 \\
\hline $\mathrm{H}$ & 0.74106 & -3.79071 & -4.99397 \\
\hline $\mathrm{H}$ & 0.01964 & -1.50612 & -5.23453 \\
\hline $\mathrm{H}$ & -1.68254 & -1.98211 & -5.17321 \\
\hline $\mathrm{H}$ & -2.46798 & 2.20593 & -5.61654 \\
\hline $\mathrm{H}$ & -0.95653 & 1.60327 & -4.92111 \\
\hline
\end{tabular}




$\begin{array}{lrrr}\mathrm{H} & -0.10292 & 3.72597 & -5.58592 \\ \mathrm{H} & -1.69214 & 4.48684 & -5.53804 \\ \mathrm{H} & -0.17301 & 4.16423 & 5.51274 \\ \mathrm{H} & 1.38189 & 3.43821 & 5.11332 \\ \mathrm{H} & 0.39935 & 1.34590 & 4.52234 \\ \mathrm{H} & -0.87067 & 1.85415 & 5.64854 \\ * & & & \end{array}$

$\%$ plots format cube

$\operatorname{dim} 1100 \operatorname{dim} 2100 \operatorname{dim} 3100$

SpinDens("dicPDIFeN2_trip.cube");

end

- Calculated Thermochemical Properties - (dicPDI)Fe( $\left.\mathbf{N}_{2}\right)$ (Calculated at 298.15 K)

Final Gibbs free enthalpy $\quad \ldots-3128.3022934755$

- Optimized Coordinates - (dicPDI)Fe( $\left.\mathbf{N}_{2}\right)$
C 3.77823369322631
1.39972628264390
$-0.64342728266882$
C 2.38228392711486
1.36432114811055
$-0.48860813229958$
N 1.74494760104903
0.15746467120945
$-0.44741410067627$
C 2.40218582858982
C 3.79865955892868
$-1.03469249535416$
$-0.55912435808275$
C 4.47648524659373
$-1.03660554955139$
$-0.71788510353042$
C 1.49938598670420
0.18930905388160
$-2.15119610917623$
$-0.75360950738852$
C 1.45802775626378
2.45868935468701
$-0.47089857645289$
C 1.92980589771915
3.88511593835851
2.07258337076962
N 0.17059295105769
C 1.99786361992269
$-3.56490318106498$
N 0.20777025438812
$-1.79709313092477$
$-0.35722369939747$
$-0.38121764098820$
$-0.22401778101190$
$-0.57108028327538$
C -0.85664496649623
3.06118460312204
$-0.30123146774106$
C -0.79037191918714
$-2.80158395900367$
$-0.05717662221757$
C -1.55519128057681
3.54713368357414
$-0.06119722451588$
C -2.60821046865376
4.45276073232494
4.87583495181708
$-1.19210454340143$
$-0.98773760105173$
C -2.96749191881110
C -2.26116900548134
4.40073701645273
0.29061113306705
C -1.19741236506794
3.49804538465081
C -1.01065557969940
$-3.20838351377932$
1.39426320391090
1.24858664737513
C -2.03295136655753
$-4.12697337663476$
1.28514966338052
C -2.83333931619452
$-4.64138141144611$
1.55884541189577
C -2.60402003348766
$-4.23900821715893$
0.54125690295588
C -1.58593257704077
$-3.32737666898924$
$-0.77059288170101$
C -1.18753522228234
C -0.45062110859134
3.13142689935649
3.01559775642805
5.57728021403121
H $\quad-3.79454868146058$
C -1.42658130070454
C -0.13420785145989
$-3.08116413209381$
$-2.71376339135032$
C -2.29131861528992
C -1.70953553566218
2.41748298132199
2.39931401044679
$-1.11183817200711$
$-2.60682455890666$
2.48035880138306
0.42955192887835
$-2.61105871422010$
2.42363920536038
$-3.42060059812192$
H $\quad-0.33892934783460$
2.43331231127461
$-4.84103908102109$
$-2.54670268822688$ 


\begin{tabular}{|c|c|c|c|}
\hline & -1.29895247933355 & 2.24031576101825 & \\
\hline & .35737845104629 & 13766158779049 & \\
\hline & 3228933556259 & 26868905 & 030 \\
\hline & 0.21110948257781 & 4.12334983402469 & \\
\hline & .80227175147099 & -3.78141221528760 & 3.03687788582556 \\
\hline & .35356059603477 & -3.07755994730846 & 4.28282951694451 \\
\hline & 0.14536420407598 & -2.28328647035903 & 4.84626185714606 \\
\hline & 6593109116961 & -2.15242457808269 & \\
\hline & 301289368869 & 456 & \\
\hline & 973027300777 & 59429779260 & 26938 \\
\hline & -0.70376871162996 & -2.13958423076008 & -4.6 \\
\hline & -0.78805203088665 & -1.80866278394399 & 886305252177 \\
\hline & -0.12975232728371 & 0.13495418030812 & \\
\hline & -1.96261863570227 & 0.11607127687784 & -0.05203 \\
\hline & 1983 & & \\
\hline & & & \\
\hline & 5.5623220 & 0.20 & $-0 . \varepsilon$ \\
\hline & 2573637 & 4.14 & -1.3 \\
\hline & 1.112 & 4.589 & \\
\hline & 2.71 & 4.0573 & 0.37 \\
\hline & 2.42 & -3.76 & \\
\hline & & & \\
\hline & 1.19 & -4.2 & \\
\hline & 5794 & 7824 & 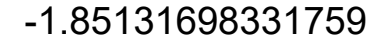 \\
\hline & -2.54117178719885 & 57344 & 96021529 \\
\hline & -2.20650153125283 & & \\
\hline & -3.23 & -4.6 & -1. \\
\hline & & & \\
\hline & -2.4 & -3.1 & -3 \\
\hline & 0.513 & -1.91 & \\
\hline & & -5.3 & 9720 \\
\hline & -2.53577967586100 & & 00871 \\
\hline & -3.222829383 & 3.00 & 70277 \\
\hline & -1.0 & & \\
\hline & & 4.2 & \\
\hline & & & \\
\hline & -2.19 & 2.823 & 6267 \\
\hline $\mathrm{H}$ & 175875570 & 1.26 & 721795 \\
\hline & 8246 & & 3. \\
\hline & 1.18 & 4.43 & \\
\hline & & & \\
\hline & 1.586 & -4.11 & \\
\hline & -1.77081359951282 & -2.74 & 93970 \\
\hline $\mathrm{H}$ & -1.20624472180317 & -1.11 & 592098067596 \\
\hline & 0.27391813490073 & -4.435 & -2.79665177303365 \\
\hline & -1.23550077084100 & -5.1 & -3.37938333281724 \\
\hline & 0.222 & -1.65 & -2.7 \\
\hline & -1.373 & -0.899 & -2.99241896946224 \\
\hline & 0.27284327802239 & 4.60192192658749 & -3.38117857318522 \\
\hline & -3.06684767009175 & 0.10641858695613 & 0.05319355220484 \\
\hline & 2.15369589731124 & -2.38188419438960 & 3.97751625756983 \\
\hline & 1.79411477654648 & -3.77212920501277 & 5.01621238762106 \\
\hline
\end{tabular}




$\begin{array}{llcc}\text { H } & -0.31516485798428 & -2.82145183186021 & 5.68967485722745 \\ \text { H } & 0.45676853354648 & -1.30247653380769 & 5.23876302295031 \\ \text { H } & -0.89355327818442 & -4.16827147676448 & -5.54299808123819 \\ \text { H } & 0.73657418101556 & -3.78472273031796 & -4.99634069378823 \\ \text { H } & 0.01474462099792 & -1.49997481396089 & -5.23392090784995 \\ \text { H } & -1.68738639313210 & -1.97599646009266 & -5.17115849033750 \\ \text { H } & -2.46674809878899 & 2.20032328719643 & -5.61610984608851 \\ \text { H } & -0.95613526393652 & 1.59681876459497 & -4.91958527372482 \\ \text { H } & -0.09993993910710 & 3.71781235331378 & -5.58607761584502 \\ \text { H } & -1.68840707820483 & 4.48043348925326 & -5.54004424721950 \\ \text { H } & -0.17520453568645 & 4.17410270463324 & 5.51110467050017 \\ \text { H } & 1.37941577195471 & 3.44626752021977 & 5.11395833702753 \\ \text { H } & 0.39571239687347 & 1.35335097421125 & 4.52668168900607 \\ \text { H } & -0.87444872551706 & 1.86476925753085 & 5.65131781708620\end{array}$

\section{- DFT Input File $-\mathbf{N}_{2}$}

! RKS B3LYP RIJCOSX def2-SVP def2/J Normalprint SlowConv TightSCF NumFreq Opt Pal8 UCO

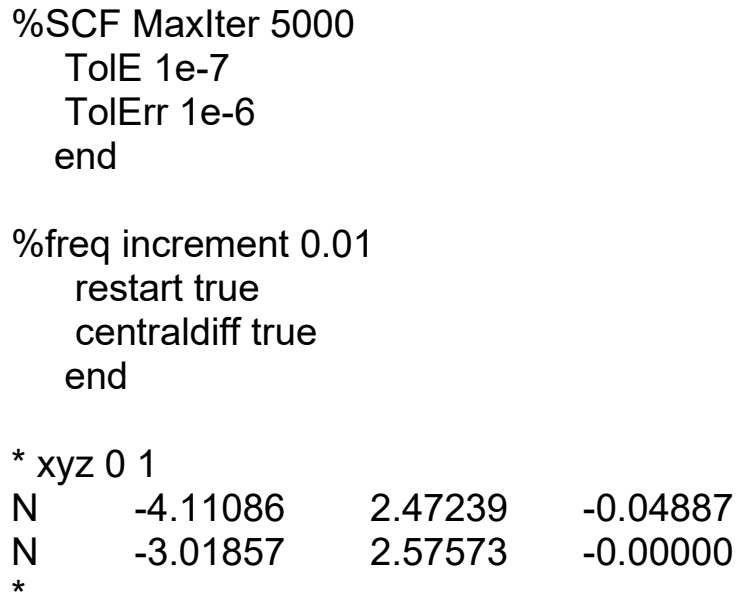

end

- Calculated Thermochemical Properties $-\mathrm{N}_{2}$ (Calculated at 298.15 K)

Final Gibbs free enthalpy $\quad \ldots \quad-109.387128353725$

- Optimized Coordinates $-\mathrm{N}_{2}$
N -4.11199814553505
2.47228232167319
$-0.04892092161633$
N -3.01743185446495
2.57583767832681
0.00005092161633 
- MECP for $\mathbf{N}_{2}$ Dissociation from (dicPDI)Fe $\left(\mathbf{N}_{2}\right)\left(\eta^{2}\right.$-propylene)
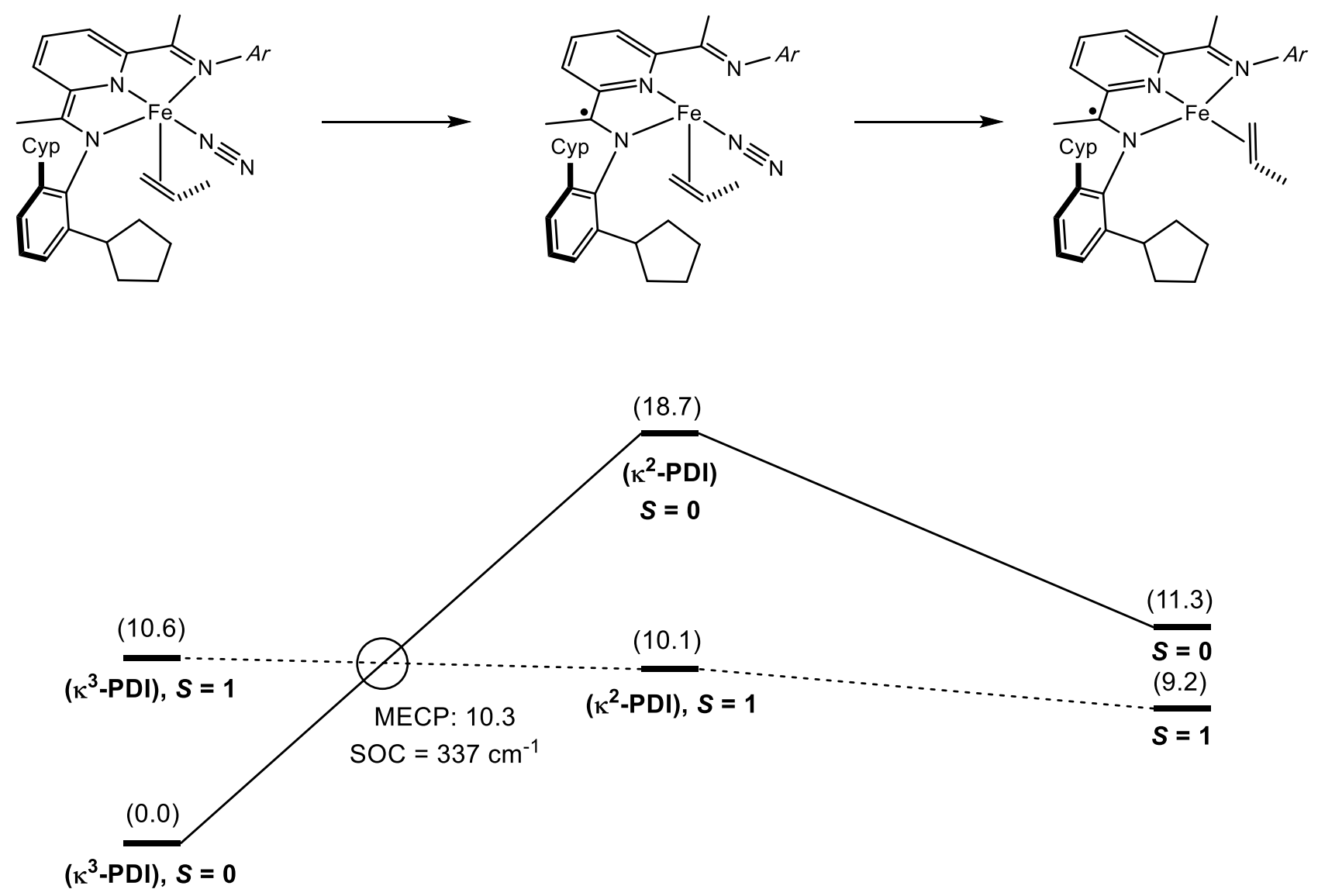

Figure S35. Relative Gibbs free energy values for potential intermediates along the pathway for $\mathrm{N}_{2}$ dissociation from (dicPDI)Fe( $\left.\mathbf{N}_{2}\right)\left(\eta^{2}\right.$-propylene). Values given in $\mathrm{kcal} \cdot \mathrm{mol}^{-1}$. Potential for spin transition during imine arm dissociation followed by $\mathrm{N}_{2}$ dissociation. SOC constant and MECP level crossing was calculated according to methods detailed by Holland and co-workers..$^{30} \mathrm{SOC}$ constant of $337 \mathrm{~cm}^{-1}$ matches well with the reported constant for free $\mathrm{Fe}^{+}$ion $\left(345 \mathrm{~cm}^{-1}\right)^{31}$ which indicates that a spin transition is possible via spin-orbit coupling. 


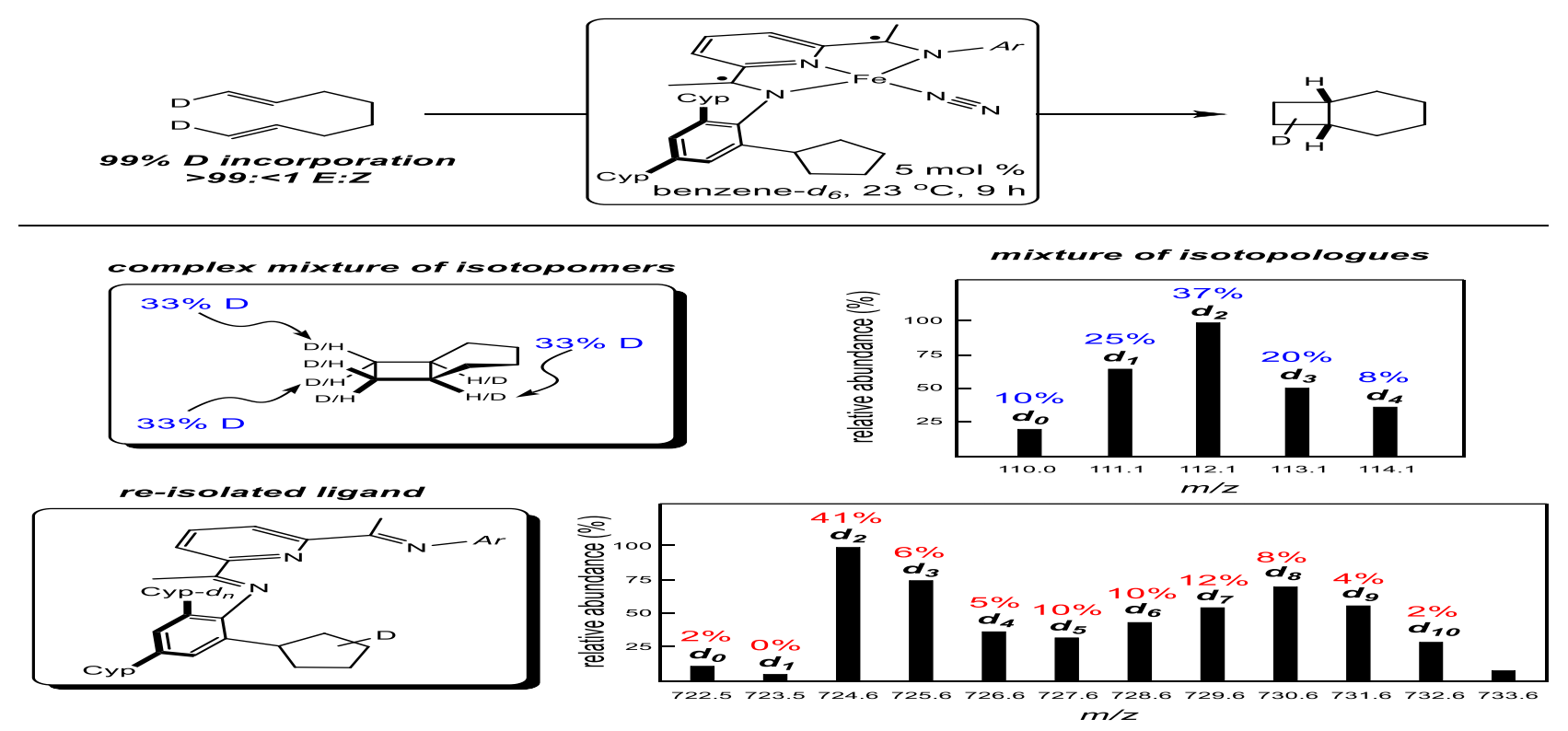

Figure S36. Ground-state energy reaction pathways after reductive elimination from a PDI iron metallacycle. All complexes are in an $S=1$ spin state.

DFT methods were used to explore from what iron compounds undergo cyclometallation. Because reductive elimination most likely occurs intramolecularly from an $S=1 \mathrm{PDI}$ iron metallacycles, a coordinatively unsaturated iron complex should be generated prior to reductive elimination. As depicted in Figure S35, subsequent to reductive elimination of a truncated (dicPDI)Fe(2,3-dimethylmetallacyclopentane), rotation of an imine aryl group to-wards the iron center produced an agostic $\mathrm{C}-\mathrm{H}$ interaction from a $\mathrm{C}-\mathrm{H}$ bond at the 2-position of a cyclopentyl group. Geometry optimizations at multiple electronic configurations produced the $\mathrm{C}-\mathrm{H}$ agostic bond, however it con-verged to an $S=1$, broken symmetry $(2,2)$ solution. Con-straining the imine aryl groups from rotating and preventing the $\mathrm{C}-\mathrm{H}$ agostic bond leads to a complex $10.1 \mathrm{kcal} \cdot \mathrm{mol}-1$ higher in energy than with the agostic interaction. As it is well-known that an agostic $\mathrm{C}-\mathrm{H}$ bond is a prerequisite to many $\mathrm{C}-\mathrm{H}$ activation mechanisms, it is highly likely that cyclometallation, and therefore the entryway to catalyst

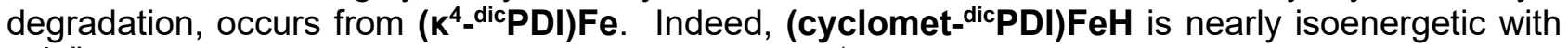
( $\mathbf{K}^{4}$ _dicPDI)Fe, with the latter being only $2.8 \mathrm{kcal} \cdot \mathrm{mol}^{-1}$ higher in energy. From this cylcometallated complex, reversible hydride insertion into alkenes and $\beta-\mathrm{H}$ elimination (which can scramble deuteria if they are present in the alkene) and ligand dehydrogenation to yield (dehydro-dic $\mathbf{P D I}) \mathbf{F e}\left(\mathbf{N}_{\mathbf{2}}\right)$ are possible pathways to sequester the catalyst from productive reactivity and lead to deactivation.

( $\mathbf{K}^{4}$-dicPDI)Fe can also take productive pathways to turnover the catalytic cycle: e.g. reacting with dinitrogen to produce (dicPDI)Fe( $\left.\mathbf{N}_{2}\right)$ and reacting with an alkene to produce (dicPDI)Fe( $\mathbf{n}^{2}$-alkene) (Figure S35). Both pathways are enthalpically driven $\left(\Delta \mathrm{G}=-17.7 \mathrm{kcal} \cdot \mathrm{mol}^{-1}\right.$ and $-7.6 \mathrm{kcal} \cdot \mathrm{mol}^{-1}$ respectively), however cyclometallation likely has a low entropic cost, as the reaction is unimolecular and requires minimal reorganization compared to ligand substitution and re-positioning of the imine aryl groups.

During both inter- and intramolecular iron-catalyzed [2+2] cycloadditions of alkenes, it can be surmised that the same ( $\mathbf{K}^{4}$-tricPDI)Fe intermediate is formed, further supported by the similar amounts of ligand cyclometallation observed in both classes of reactions. In the intermolecular reaction, ( $\mathbf{K}^{4}{ }_{-}$tricPDI)Fe can react with either dinitrogen or alkene (likely depending on $\mathrm{N}_{2}$ pressure), while in the intramolecular case, $\left(\mathbf{K}^{4}\right.$ tric $\left.\mathbf{P D I}\right) \mathbf{F e}$ preferentially reacts with an $\alpha, \omega$-diene. This higher 
binding constant compared to dinitrogen/a-olefins is likely a result of the chelate effect which helps to maintain a catalyst-substrate complex throughout the reaction.

In the iron-catalyzed [2+2] cycloaddition of propylene, while the reaction rate increased with lower $\mathrm{N}_{2}$-pressure, catalyst degradation appeared to also increase. With a lower concentration of $\mathrm{N}_{2}$ in the reaction, turnover of ( $\mathbf{K}^{4}$-tric $\left.P D I\right) F e$ to ( $\left.{ }^{\text {tric }} \mathbf{P D I}\right) \mathbf{F e}\left(\mathbf{n}^{2}\right.$-propylene) is preferred and the cycle can remain on the $S=1$ spin surface where there is less iron catalyst tied up at the catalyst resting state $(S=0$ surface), leading to a fast initial rate of reaction. Low $\mathrm{N}_{2}$ concentration, however, also causes ligand $\mathrm{C}-\mathrm{H}$ activation of ( $\boldsymbol{k}^{4}{ }_{-}$tric $\left.\mathbf{P D I}\right) \mathrm{Fe}$ to become more favorable and leads to faster rates of catalyst dehydrogenation, as the binding affinity of alkenes to (tricPDI)Fe is relatively low (manifested in a faster rate of catalyst death at $0 \mathrm{~atm} \mathrm{~N}_{2}$ compared with $1 \mathrm{~atm}$ ). This demonstrates how altering reaction conditions can change which reaction pathways ( $\left.\mathbf{K}^{4}{ }^{\text {tric }} \mathbf{P D I}\right) \mathbf{F e}$ accesses during catalyst turnover.

\section{References}

1. Pangborn, A. B.; Giardello, M. A.; Grubbs, R. H.; Rosen, R. K.; Timmers, F. J. Safe and Convenient Procedure for Solvent Purification. Organometallics 1996, 15, 1518-1520.

2. Sevov, C. S.; Zhou, J.; Hartwig, J. F. J. Am. Chem. Soc. 2014, 136, 3200-3207.

3. Schmidt, V. A.; Hoyt, J. M.; Margulieux, G. W.; Chirik, P. J. J. Am. Chem. Soc. 2015, 137 (24), 79037914.

4. Hoyt, J. M.; Schmidt, V. A.; Tondreau, A. M.; Chirik, P. J. Science 2015, 349 (6251), 960-963.

5. Hoyt, J. M.; Sylvester, K. T.; Semproni, S. P.; Chirik, P. J. J. Am. Chem. Soc. 2013, 135 (12), $4862-$ 4877.

6. Kuppuswamy, S.; Wofford, J. D.; Joseph, C.; Xie, Z.; Ali, A. K.; Lynch, V. M.; Lindahl, P. A.; Rose, M. J. Inorg. Chem. 2017, 56, 5998-6012.

7. Ion Prisecaru, WMOSS4 Mössbauer Spectral Analysis Software, www.wmoss.org, 2009-2016.

8. Neese, F. ORCA: an ab initio, DFT and Semiempirical Electronic Structure Package, Version 2.8, Revision 2287; Institut für Physikalische und Theoretische Chemie, Universität Bonn: Bonn, Germany, 2010

9. Perdew, J. P. Density-functional approximation for the correlation energy of the inhomogeneous electron gas. Phys. Rev. B 1986, 33, 8822-8824.

10. Perdew, J. P. Erratum: Density-functional approximation for the correlation energy of the inhomogeneous electron gas. Phys. Rev. B 1986, 34, 7406.

11. Lee, C. T.; Yang, W. T.; Parr, R. G. Development of the Colle-Salvetti correlation-energy formula into a functional of the electron density. Phys. Rev. B 1988, 37, 785-789.

12. Neese, F.; Solomon, E. I. In Magnetism: From Molecules to Materials; Miller, J. S.; Drillon, M., Eds.; Wiley: New York, 2002; Vol. 4, p 345.

13. Schäfer, A.; Horn, H.; Ahlrichs, R. Fully optimized contracted Gaussian basis sets for atoms Li to Kr. J. Chem. Phys. 1992, 97, 2571-2577. 
14. Schäfer, A.; Huber, C.; Ahlrichs, R. Fully optimized contracted Gaussian basis sets of triple zeta valence quality for atoms Li to Kr. J. Chem. Phys. 1994, 100, 5829-5835.

15. Weigend, F.; Ahlrichs, R. Balanced basis sets of split valence, triple zeta valence and quadruple zeta valence quality for $\mathrm{H}$ to Rn: Design and assessment of accuracy. Phys. Chem. Chem. Phys. 2005, 7, 3297-3305.

16. Eichkorn, K.; Weigend, F.; Treutler, O.; Ahlrichs, R. Auxiliary basis sets for main row atoms and transition metals and their use to approximate Coulomb potentials. Theor. Chem. Acc. 1997, 97, 119-124.

17. Eichkorn, K.; Treutler, O.; Öhm, H.; Häser, m.; Ahlrichs, R. Auxiliary basis sets to approximate Coulomb potentials. Chem. Phys. Lett. 1995, 240, 283-289.

18. Eichkorn, K.; Treutler, O.; Öhm, H.; Häser, M.; Ahlrichs, R. Auxiliary basis sets to approximate Coulomb potentials. Chem. Phys. Lett. 1995, 242, 652-660.

19. Neese, F.; Wennmohs, F.; Hansen, A.; Becker, U. Efficient, Approximate and parallel Hartree-Fock and hybrid DFT calculations. A 'chain-of-spheres' algorithm for the Hartree-Fock exchange. Chem. Phys. 2009, 356, 98-109.

20. Kossmann, S.; Neese, F. Comparison of two efficient approximate Hartee-Fock approaches. Chem. Phys. Lett. 2009, 481, 240-243.

21. Neese, F. An improvement of the resolution of the identity approximation for the formation of the Coulomb matrix. J. Comput. Chem. 2003, 24, 1740-1747.

22. Ginsberg, A. P. Magnetic exchange in transition metal complexes. 12. Calculation of cluster exchange coupling constants with the Xa-scattered wave method. J. Am. Chem. Soc. 1980, 102, 111-117.

23. Noodleman, L.; Peng, C. Y.; Case, D. A.; Mouesca, J. M. Orbital interactions, electron delocalization and spin coupling in iron-sulfur clusters. Coord. Chem. Rev. 1995, 144, 199-244.

24. Kirchner, B.; Wennmohs, F.; Ye, S.; Neese, F. Theoretical bioinorganic chemistry: The electronic structure makes a difference. Curr. Opin. Chem. Biol. 2007, 11, 134-141.

25. Neese, F. J. Definition of corresponding orbitals and the diradical character in broken symmetry DFT calculations on spin coupled systems. Phys. Chem. Solids 2004, 65, 781-785.

26. Pettersen, E. F.; Goddard, T. D.; Huang, C. C.; Couch, G. S.; Greenblatt, D. M.; Meng, E. C.; Ferrin, T. E. UCSF Chimera-A visualization system for exploratory research and analysis. J. Comput. Chem. 2004, 25, 1605-1612.

27. Brinker, U. H.; Lin, G.; Xu, L.; Smith, W. B.; Mieusset, J. J. Org. Chem. 2007, 72, $8434-8451$

28. Metzger, P.; Cabestaing, C.; Casadevall, E.; Casadevall, A. Organic Magnetic Resonance, 1982, 19, $144-147$

29. Singleton, D. A.; Thomas, A. A. J. Am. Chem. Soc. 1995, 117 (36), 9357-9358.

30. Bellows, S. M.; Cundari, T. R.; Holland, P. L. Spin Crossover during $\beta$-Hydride Elimination in HighSpin Iron(II)- and Cobalt(II)-Alkyl Complexes. Organometallics 2013, 32 (17), 4741-4751.

31. Dunn, M. Spin-orbit Coupling In the First and Second Transition Series. Trans. Faraday Soc.1961,57, 1441-1444 